\title{
Transcriptomics close to my heart
}

Citation for published version (APA):

Verheijen, M. (2019). Transcriptomics close to my heart: advanced models \& methods for toxicogenomics research illustrated by anthracycline-induced cardiotoxicity. [Doctoral Thesis, Maastricht University]. ProefschriftMaken Maastricht. https://doi.org/10.26481/dis.20190517mv

Document status and date:

Published: 01/01/2019

DOI:

10.26481/dis.20190517mv

Document Version:

Publisher's PDF, also known as Version of record

\section{Please check the document version of this publication:}

- A submitted manuscript is the version of the article upon submission and before peer-review. There can be important differences between the submitted version and the official published version of record.

People interested in the research are advised to contact the author for the final version of the publication, or visit the DOI to the publisher's website.

- The final author version and the galley proof are versions of the publication after peer review.

- The final published version features the final layout of the paper including the volume, issue and page numbers.

Link to publication

\footnotetext{
General rights Owners
rights.

- You may freely distribute the URL identifying the publication in the public portal. please follow below link for the End User Agreement:

www.umlib.nl/taverne-license

Take down policy

If you believe that this document breaches copyright please contact us at:

repository@maastrichtuniversity.nl

providing details and we will investigate your claim.
}

Copyright and moral rights for the publications made accessible in the public portal are retained by the authors and/or other copyright owners and it is a condition of accessing publications that users recognise and abide by the legal requirements associated with these

- Users may download and print one copy of any publication from the public portal for the purpose of private study or research.

- You may not further distribute the material or use it for any profit-making activity or commercial gain

If the publication is distributed under the terms of Article $25 \mathrm{fa}$ of the Dutch Copyright Act, indicated by the "Taverne" license above, 


\title{
Transcriptomics close to my heart
}

Zduanced models \& methods for toxicogenomics research illustrated by anthracycline-induced cardiotoxicity

\author{
Marcha Verheijen
}


(C) Marcha Verheijen, Maastricht, 2019

ISBN: 978-94-6380-299-4

Layout: Marcha Verheijen

Cover design: Marcha Verheijen

Cover contains samples of binary stock photo (Dreamstime.com 27314807) and my cardiac echo (many thanks to Dr. R. ter Bekke for obtaining it)

Printed by:

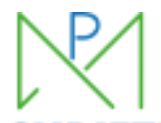

PROEFSCHRIFTMAKEN 


\section{Transcriptomics close to my heart}

Zlduanced models \& methods for toxicogenomics research

illustrated by anthracycline-induced cardiotoxicity

\section{DISSERTATION}

to obtain the degree of Doctor at Maastricht University,

on the authority of the Rector Magnificus, Prof. dr. Rianne M. Letschert

with the decision of the Board of Deans, to be defended in public

on Friday, 17th May 2019 at 10 hours

by

Maria Christina Theodora Verheijen

born on $25^{\text {th }}$ March 1987, in Amersfoort, The Netherlands 


\section{Promotor}

Prof. dr. Jos C.S. Kleinjans

\section{Co-promotor}

Dr. Florian Caiment

\section{Assessment committee}

Prof. dr C.T.A Evelo (chairman)

Prof. dr. H.J.G.M. Crijns

Prof. H.J.M. Smeets

Prof. dr. M. Vinken, Vrije Universiteit Brussel

Prof. dr. A.H. Piersma, Universiteit Utrecht

The research described in this thesis was conducted at GROW School of Oncology and Developmental Biology of Maastricht University. 


\section{Table of Contents}

Chapter 1: $\quad$ General Introduction

Chapter 2: $\quad$ DMSO induces drastic changes in human cellular

processes and epigenetic landscape in vitro

Chapter 3: $\quad$ Bringing in vitro analysis closer to in vivo:

Studying doxorubicin toxicity and associated mechanisms in 3D human microtissues with PBPKbased dose modelling

Chapter 4: Deeper than gene expression:

Analyzing differential transcript usage of sarcomeric genes in anthracycline-induced cardiotoxicity

Chapter 5: $\quad$ Assessment of microRNA \& circular RNA interactions reveals the importance for analyzing posttranscriptional mechanisms

Chapter 6: $\quad$ Summary and General Discussion 

Chapter 1

\section{General Introduction}

In part published as:

Development and regulatory application of microRNA biomarkers

Verheijen, M., et al. Biomark Med, 2015 Volume 9 (11), p. 1137-1151 
In analogy with Apollo Hekatos, the greek god with the silver bow whose arrows never missed their target, the EU/FP5 HeCaToS (Hepatic and Cardiac

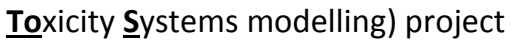

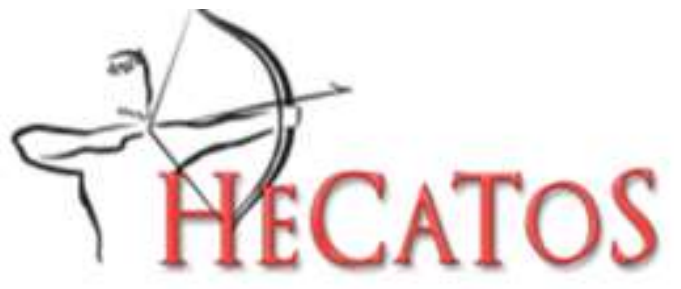
aimed to develop a flexible computer Figure 1: HeCaToS project logo simulated (in silico) prediction system aiding in drug safety assessment for humans ${ }^{[1]}$. Current drug development and safety testing procedures are time consuming, expensive and not able to detect all drug-induced toxicities, with severe side effects (adverse drug reactions) as a consequence. The adverse drug reactions also rank high as a cause for disease or even death. It would therefore be beneficial to improve the possibility to detect or predict drug-induced toxicities at an early stage of drug development $^{[2]}$.

As part of the HeCaToS project, this thesis will focus on the toxic effects of anthracyclines on a cardiac cell model through transcriptomics analysis, with the addition of epigenomics data and proteomics data for achieving more in depth analysis.

\section{Anthracyclines}
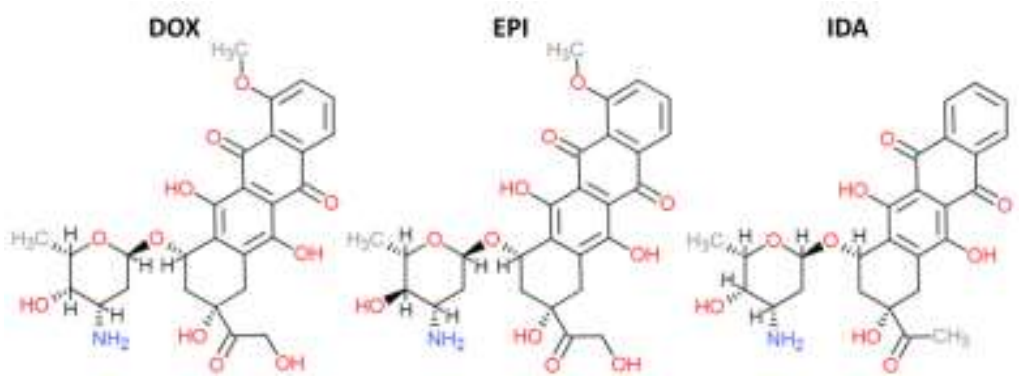

Figure 2:

Chemical structures of ANTs, obtained from PubChem ${ }^{[9]}$ and represented using ChemDoodle ${ }^{[10]}$

The anthracyclines (ANTs) were amongst the first chemotherapeutic agents to be discovered $^{[3]}$. The most commonly used ANT is Doxorubicin (DOX) ${ }^{[4]}$, which was isolated from Streptomyces peucetius var. caesius in $1967^{[5]}$. Epirubicin (EPI) and Idarubicin (IDA) are chemically produced analogs of $\mathrm{DOX}^{[6]}$. Due to the slight structural differences (Figure 2), IDA is more lipophilic with a higher cellular uptake and EPI results in less side effects, though the antitumor activity is also reduced ${ }^{[7,8]}$. 
While the use of ANTs has greatly contributed to increased cancer survival rates, a major downside is the occurrence of side effects: nausea, vomiting, alopecia, myelosuppression, stomatitis, gastrointestinal disturbances, and most especially dosedependent cardiotoxicity. Features of ANT-induced cardiotoxicity include ventricular and contractile dysfunction, resulting in decreased left ventricular ejection fraction (LVEF) which can ultimately lead to dilated cardiomyopathy (DCM) and heart failure $(\mathrm{HF})^{[11,12]}$. Two types of cardiotoxicity can be distinguished. The most prevalent is an acute cardiotoxicity, which is reversible and clinically manageable. This type occurs during treatment with a single high dose or within 2-3 days after repeated treatments ${ }^{[13,14]}$. The most concerning is the chronic cardiotoxic phenotype, which is irreversible and progressive in nature and has a poor prognosis for the patient. This type occurs between one month and several decades after treatment ${ }^{[14-18]}$. To reduce the incidence of cardiotoxicity, a maximum recommended cumulative doses (DOX: $400 \mathrm{mg} / \mathrm{m}^{2}$; EPI: $900 \mathrm{mg} / \mathrm{m}^{2} ;$ IDA: $150 \mathrm{mg} / \mathrm{m}^{2}$ ) have been established, though this is only partially effective ${ }^{[7]}$.

Even for DOX, the best studied ANT, mechanisms of action are not fully understood. The main mode of action is related to killing dividing cells. Because the rate of cell division is generally increased in tumor cells, these cells are more sensitive to the treatment. While non-tumor cells are less sensitive, DOX still affects these cells and can even accumulate in the nucleus and mitochondria of heart, liver and blood cells. Even at therapeutic doses, this may induce acute or chronic toxic side effects ${ }^{[16]}$.

Several biological mechanisms of DOX have already been identified. It is well known that DOX can bind to DNA and inhibit DNA topoisomerase to prevent unwinding. In this way, the DNA becomes inaccessible, which inhibits DNA replication, RNA transcription and protein biosynthesis ${ }^{[19,20]}$. It is generally accepted that this mechanism results in the anti-cancer effects of DOX. However, DOX can also induce the generation of reactive oxygen species $(\mathrm{ROS})^{[21-23]}$, which is mainly ascribed to cause toxic effects ${ }^{[24]}$. There are two ways through which ROS can be generated: 1 ) as a result of mitochondrial dysfunction, and 2 ) by oxidative semiquinone formation at complex 1 of the electron transport chain (ETC) during DOX metabolism. This generation of ROS 
can induce oxidative stress which may damage cells and cause cell death ${ }^{[22,25]}$. Death of non-cancer cells may be one cause for decreased cardiomyocyte function, though this may also be the result from mitochondrial dysfunction causing an imbalance in cellular energetics ${ }^{[26]}$. Overall, it is essential to unravel the molecular processes of ANTinduced cardiotoxicity in order to prevent today's cancer patients from becoming tomorrow's cardiac patients ${ }^{[7]}$.

\section{Evolution of the experimental design in toxicology}

Traditional prediction of molecular mechanisms underlying long term toxicity and toxicological risk assessment relies on animal models ${ }^{[27]}$. However, the field has to reduce the amount of animal experiments, notably due to increasing ethical pressure, but also because the concordance rate between human and rodent toxicities is only $71 \%{ }^{[28]}$. Alternative to testing in living creatures (in vivo), in vitro methods can be used in which human cells are cultured in the laboratory.

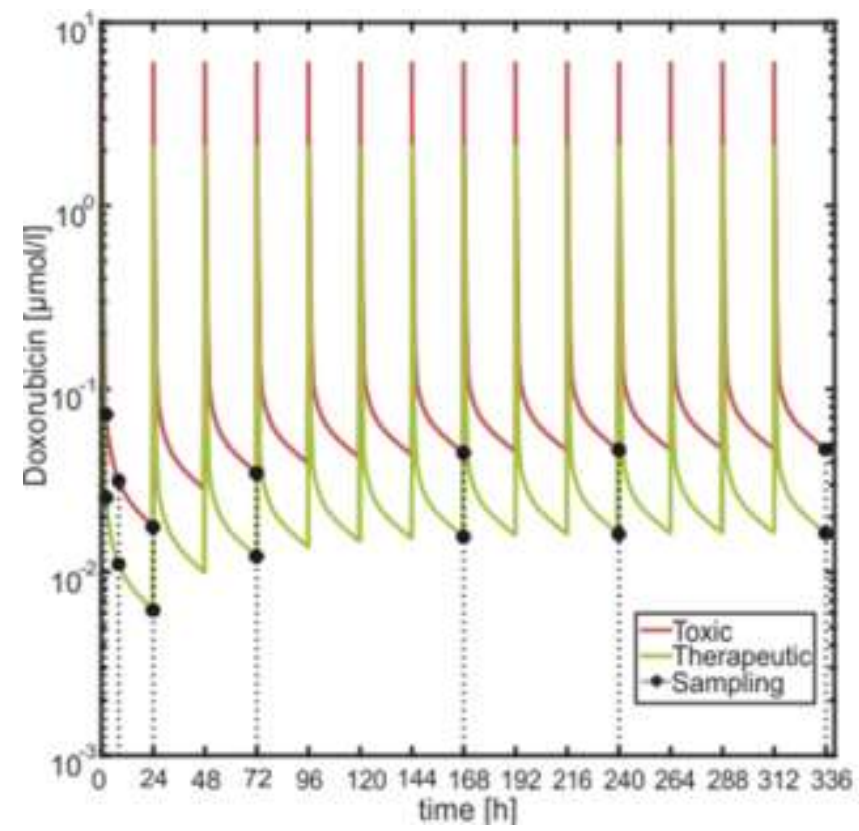

In this thesis, we use an innovative in vitro experimental design aimed to best reflect the human in vivo conditions. This design is based on a 3D spheroid cardiac model (InSphero, SWL) containing a co-culture of approximately 4000 iPSCderived human cardiomyocytes and 1000 human cardiac fibroblasts.

Figure 3: Doxorubicin PBPK model simulations. Simulations of DOX interstitial heart concentration profiles following a therapeutic (green) and toxic (red) once daily dosing regimen with experimental sampling points (black circles) $^{[33]}$ 
This model, known as a microtissue, better resembles the in vivo heart compared to the regularly used monolayer cell cultures of a single cell type ${ }^{[29-31]}$. Furthermore, instead of the traditional exposure to relatively high doses, we adopted a sophisticated exposure profile obtained through physiologically based pharmacokinetic (PBPK) modelling ${ }^{[32]}$. PBPK simulates the absorption, distribution, metabolism and excretion of a specific dose of a compound (e.g. DOX) within the human body. This enabled the design of a two weeks repetitive dosing profile which reflects the concentrations to which the human heart is exposed to during ANT treatment in vivo (Figure 3). Finally, this exposure profile was realized in the laboratory by means of 3 medium changes per day.

\section{Evolution of toxicogenomics analysis}

Toxicogenomics is the analysis of toxicological responses to a compound using genomics analysis. The central dogma of molecular biology, as declared by Francis Crick $^{[34,35]}$, describes the transfer of genetic

DNA information from DNA, through RNA, to proteins, with the latter being the functional elements within the cell ${ }^{[36-38]}$. Though often represented as one way transfer, regulatory mechanisms exist between levels.

Figure 4: Central dogma of molecular biology (blue) complemented with regulatory mechanisms (purple) and indication of entities measured using Omics techniques (green)

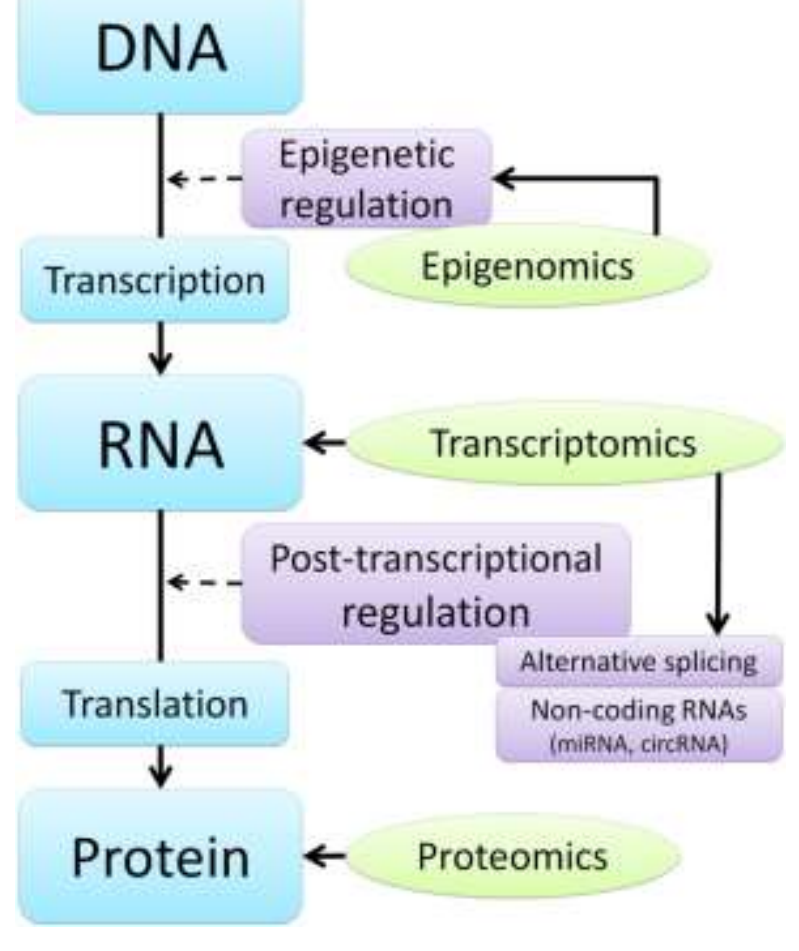


Using Omics techniques, specific aspects of the central dogma can be measured, enabling detailed analysis of the complete biological system (Figure 4). In toxicology, these methods can be applied to elucidate the reaction of cells (e.g. microtissues) in response to a toxicant (e.g. ANTs).

\section{Proteomics}

Proteins are the functional elements within a cell and are responsible for most cellular functions. Proteomics is the measurement of proteins abundances. For this thesis, we have used high-sensitivity protein mass spectrometry (MS). A global proteomics approach, as applied in this thesis, uses relative quantification between two or more proteomes to facilitate the discovery of differentially altered proteins between exposures ${ }^{[39]}$. A crucial step is the annotation of proteins, which is most often accomplished through matching the MS spectra to peptide profiles and then to proteins using database searching approaches ${ }^{[39]}$. However, MS-based proteomics is not able to detect the exhaustive landscape of all proteins expressed in the samples, resulting in missing values. A source for missing values comes from the difficulty in detecting low-abundance proteins, because the current detection limit is 3 orders of magnitude lower than the most abundant protein in the sample ${ }^{[39,40]}$. Missing values are also obtained when there is a difference in amino acid sequence (e.g. due to alternative splicing) between the sample and the database ${ }^{[41]}$. Therefore, it is not unusual for a proteomics data-set to contain $20-50 \%$ of missing peptide values ${ }^{[41]}$. Overall, typically a few thousands proteins can be quantified, which only covers a small portion of the human proteome ${ }^{[42,43]}$. Therefore, in this thesis, we used this technique for validation of findings obtained through transcriptomics analysis.

\section{Transcriptomics}

Transcriptomics is the measurement of all RNAs present within a cell. For this thesis, we have used high-throughput next generation sequencing ${ }^{[36]}$. This technique provides insight into the gene expression of the complete genome. Analysis of changes in mRNA levels are assumed to induce changed protein abundances which lead to a different phenotype ${ }^{[38,42]}$. The abundance of an mRNA can indeed predict whether the protein 
can be detected within the cell ${ }^{[43]}$. However, the correlation between gene expression and protein abundance is notoriously poor due to regulatory mechanisms acting on the translation of RNAs into proteins ${ }^{[36,37,44]}$. Therefore, hypothesis gained from geneexpression analysis need to be validated, for example with targeted protein analysis methods.

While RNA sequencing technologies are mostly applied for gene expression analysis, it is also capable of measuring post-transcriptional mechanisms such as alternative splicing and regulation through non-coding RNAs ${ }^{[45]}$. In this thesis, we aim to incorporate these mechanisms during data analysis because we postulate that it could significantly impact the understanding of biological mechanisms during transcriptomics analysis, which would greatly benefit not only toxicogenomics research but genomics research in general.

\section{Alternative splicing - isoform analysis}

RNA splicing, a post-transcriptional process necessary to form a mature mRNA, was discovered in the late 1970s. Constitutive splicing is the process of removing introns from a RNA precursor, and joining the exons together to form a mature mRNA ${ }^{[46]}$. In the case of alternative splicing, a single RNA precursor can give raise to multiple transcript variants by inclusion and exclusion of exons in different combinations. This significantly increases the diversity of the transcriptome ${ }^{[46,47]}$. While these transcripts may be closely related to each other, they can comprise non-coding variants or be translated into proteins with distinct functions ${ }^{[48]}$. Because $92-94 \%$ of human genes are estimated to be subjected to alternative splicing ${ }^{[48]}$, analysis of this post-transcriptional mechanism may facilitate elucidating toxicological mechanisms in more depth and may possibly provide new biomarkers for early detection of toxicity in vivo.

\section{Non-coding RNAs}

Only around $1.5 \%$ of the transcribed RNAs encode proteins, the remainder are the non-protein coding RNAs which may function in regulatory processes ${ }^{[45,49]}$. Based on the length and structure of the transcript, the non-protein coding RNAs can be divided 
in short non-coding RNAs (including microRNAs), long non-coding RNAs and circular $\mathrm{RNAs}^{[50]}$. In this thesis, we focus on the regulatory actions of microRNAs and circular RNAs which can inhibit the translation of mRNAs into proteins.

\section{MicroRNAs}

In 1993, the discovery of the first microRNA (miRNA), lin-4 in C. elegans, opened up a new field of research ${ }^{[45]}$. MicroRNAs (miRNAs) are small ( $\left.\approx 22 \mathrm{nt}\right)$, evolutionarily conserved, endogenously expressed single-stranded RNA molecules (for review see Kaikkonen et al. ${ }^{[51]}$ ). It is estimated that as much as $30-50 \%$ of mammalian genes exhibit miRNA-mediated regulation through complementary binding of the miRNA to the mRNA target ${ }^{[51]}$. One miRNA can regulate up to hundreds of different mRNA targets and one single mRNA target can be regulated by multiple miRNAs, adding a new dimension to the already complex network of gene regulation ${ }^{[2,52]}$.

MiRNAs exert their function mostly by preventing protein synthesis through interfering with mRNA (Figure 5). However, elucidating the actual mechanisms by which miRNAs act is quite a challenge and there is no consensus about their mode of action (for review: ${ }^{[53-55]}$ ). They can silence gene expression at the posttranscriptional level by translational repression, mRNA degradation, and/or mRNA sequestration, but it is still unclear if and how these processes are linked ${ }^{[22,54,56]}$. In humans, miRNAinduced mRNA degradation is a rare event. Rather miRNAs inhibit mRNA translation by binding to the $3^{\prime}$ untranslated region ${ }^{[49]}$. This binding depends on complementary base pairing of 2-8 nucleotides in the 5'end of the miRNA, which is known as the "seed" region $^{[45,57]}$. Next to base pairing, translational inhibition is dependent on the expression levels of both the miRNA and it mRNA target ${ }^{[45]}$. Additionally, miRNAs do not only act as translational repressors. Furthermore, they are able to activate gene expression through a link with the epigenetic machinery ${ }^{[58]}$. Finally, counter-regulation by circular RNAs can sequester miRNAs away from their target, thereby preventing their silencing effects ${ }^{[2,59]}$. 


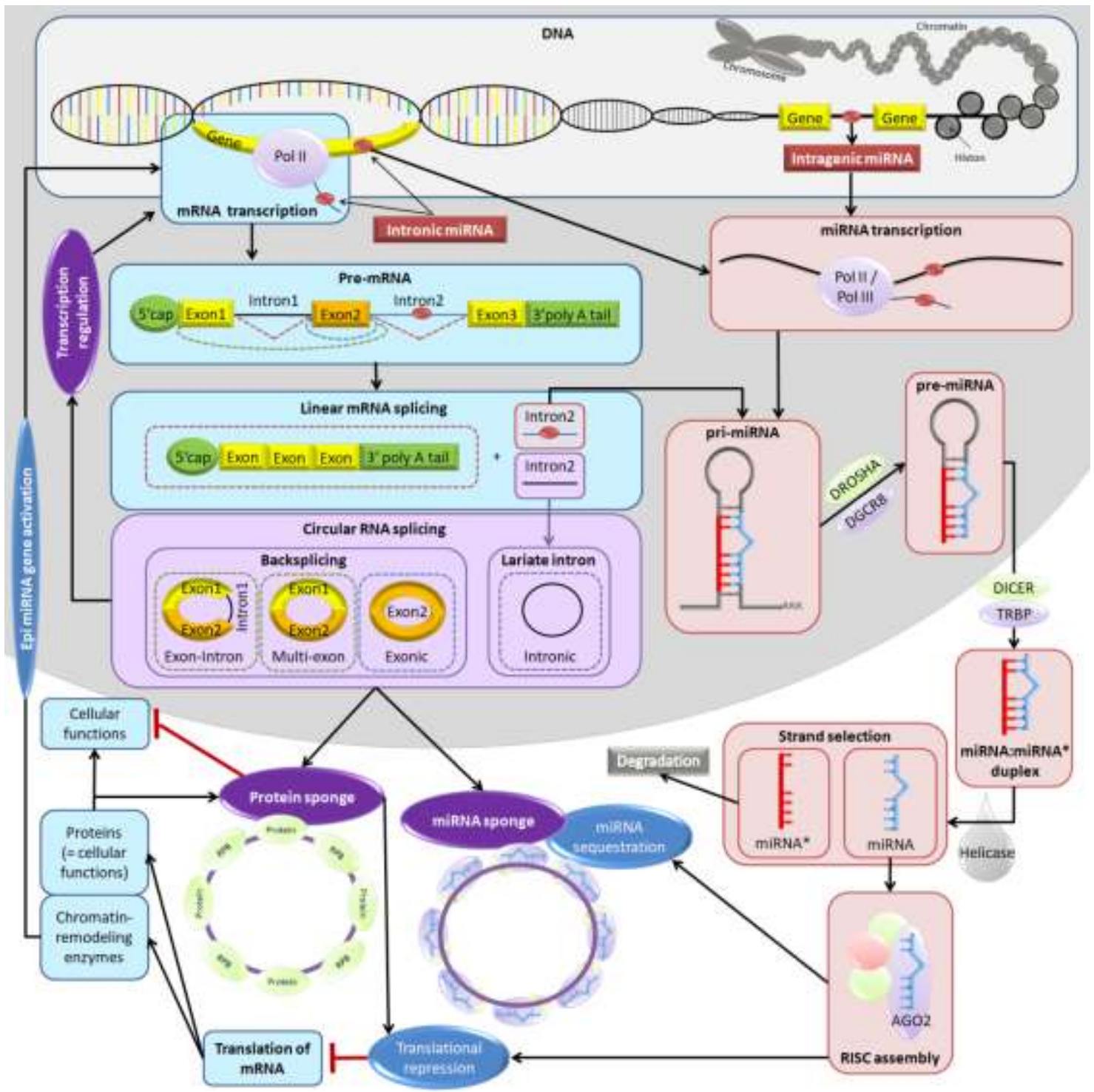

Figure 5: Biogenesis and regulatory functions of miRNA and circRNAs. Before a miRNA can exert its regulatory role within the cell, a primary miRNA (pri-miRNA) is initially transcribed from the genome by polymerase II or polymerase III and processed by DROSHA into a precursor miRNA (pre-miRNA). After being transported to the cytoplasm, the pre-miRNA is cleaved by DICER into mature miRNAs of which one will be incorporated into the RNA-induced silencing complex (RISC). Within this miRISC complex, mature miRNAs are responsible for target recognition via the Argonaute 2 protein (AGO2), through seed regions that are usually located in the 3'UTR of the targeted mRNA and involving often multiple binding sites. Binding of miRNA to a mRNA results in translation inhibition, thereby influencing cellular functions, which also includes epigenetic regulatory mechanisms) (for review: $\left.{ }^{[56,60-62]}\right)^{[2]}$. Furthermore, miRNA can interact with circRNAs. CircRNAs are produced during mRNA splicing, either through back splicing or resulting from lariat introns. Once created, they can bind to promotors to regulate gene transcription, act as sponges for miRNAs to prevent translation inhibition, act as sponges for RNA binding proteins (RBPs) to interfere with RNA splicing and translation, or act as protein decoy to interfere with other cellular functions (for review: ${ }^{[50,59,63-68]}$ ) 


\section{Circular RNAs}

As described above, splicing of a pre-mRNA results in a multitude of mRNA transcripts. While many of these are linear variants, circular variants can also be created. Circular RNAs (circRNAs) are evolutionary conserved non-coding RNAs with a closed continuous loop structure without 5'cap or 3'tail. Due to circularization, circRNAs are resistant to degradation by RNA exonucleases making them relatively stable and abundant in the cytoplasm ${ }^{[66,68]}$. Though they were first discovered in plants in $1976^{[69]}$, they were thought to be waste products of the RNA splicing machinery ${ }^{[50,68]}$. However, research by Salzman et al. in $2012^{[70]}$ and Hansen et al. ${ }^{[71]}$ in 2013 reestablished the interest in circRNAs.

CircRNAs are generally classified into 3 groups: 1) exonic circRNAs which contain 1 or more exons, 2) intronic circRNAs formed from intronic lariats, and 3) retained-intron circRNAs or exon-intron-circRNA made up of both introns and exons ${ }^{[65,68]}$. Exonic and retained-intron circRNAs are formed through back splicing of exons while intronic circRNAs are generated from lariat introns through reverse complementary motifs or aided by RNA binding proteins (RBPs) (Figure 5$)^{[49,50]}$.

Recent reviews summarize the current knowledge of circRNA function ${ }^{[50,59,63-68]}$. They can act both transcriptionally and post-transcriptionally. First, the biogenesis of circRNAs competes with splicing of linear RNAs, thereby downregulating the amount of protein coding mRNAs. Second, circRNAs can function as miRNA sponges. MiRNAs can bind to circRNAs containing miRNA seed regions. Because of the increased stability of the circRNAs, the miRNAs are sequestered and can no longer interfere with translation of linear mRNAs. Third, circRNAs bind to proteins to form an RNA-protein complex, thereby acting as protein decoys. The complex can disrupt protein-protein interactions to interfere with cellular function, but it can also modify the cellular destination, sequestering the proteins in the cytoplasm. Fourth, circRNAs bound to RBPs can regulate post-transcriptional events such as splicing and protein translation, but they 
can also inhibit transcription. Fifth, nuclear circRNAs can promote the transcription of their parental genes through binding at transcription sites. Finally, circRNAs can be excreted through extracellular vehicles and may therefore be involved in cell-cell communication.

Overall, the incorporation of miRNA and circRNA regulatory actions in transcriptomics analysis may result in more biologically relevant conclusions and increased understanding of toxicological processes.

\section{Epigenomics}

Finally, gene expression can also be regulated through epigenetic marks in the DNA which prevent the transcription of a gene into RNA. Epigenetics literally means "above" or "on top of" genetics. It refers to heritable changes in gene function that cannot be explained by changes in DNA sequence ${ }^{[72,73]}$. The most studied epigenetic mark is DNA methylation, in which a methyl group is covalently bound to a cytosine base of the DNA sequence ${ }^{[74]}$. When this mark is located in the promotor region of a gene, it functions as a translational repressor. Additionally, when located in intergenic regions, these marks help in maintaining genome stability through silencing of retrotransposable elements ${ }^{[75,76]}$. Within toxicogenomics, whole genome methylation profiling by MeDIP-seq can be used to assess compound-induced epigenetic alterations, which may cause decreased gene expression and altered cellular functions.

While studying epigenetic changes does not improve the interpretation of transcriptomics analysis (as analysis of post-transcriptional mechanism does), it creates the opportunity to broaden our research horizon and may identify causes of geneexpression changes. Overall, future toxicological research can greatly benefit form a cross-omics approach, in which data is obtained from all omics platforms to obtain a detailed and complete overview of biological changes induced by a toxicant. 


\section{Aim \& Outline of the thesis}

Due to immense technological advancements over the last decade, biomedical science has evolved towards more sensitive high-throughput techniques and towards new areas of research, including regulatory mechanisms affecting transcription and translation. We hypothesize that the use of an experimental design better reflecting the human in vivo conditions combined with analysis of post-transcriptional mechanisms would aid the evolution of toxicogenomics research in order to improve drug safety assessments in the future.

Within toxicology, the experimental design typical includes the use of a solvent like Dimethyl sulfoxide (DMSO) in order make a stable stock solution of the investigated compound. However, in the 1960s DMSO was researched for medical use due to its biological effects. In Chapter 2, we evaluated the impact of 0.1\% DMSO exposure by analyzing changes in protein abundances, gene expression and methylation profiles. Furthermore, Chapter 3 contains the validation of our innovative in vitro experimental design utilizing Human 3D cardiac microtissues and exposure to PBPK-based repetitive dosing profiles of DOX. We assessed whether our model was able to retrieve the known mechanisms of DOX through pathway analysis and in depth analysis of mitochondrial dysfunction.

In order to extract more biological information from the RNA sequencing data, we moved from standard gene expression analysis to analysis of gene isoforms created during alternative splicing. In Chapter 4, we used ANT exposed microtissues to assess gene expression and differential transcript usage (DTU) of sarcomeric genes that are involved in heart failure. Results obtained in vitro were validated in vivo through assessment of DTU in human heart biopsies of patients with cardiac dysfunction. In Chapter 5, we take the analysis of post-transcriptional mechanisms to the next level by designing a scoring system for genome-wide analysis of post-transcriptional miRNA and circRNAs interactions on mRNA translation. Finally, Chapter 6 contains the summary and discussion on the final outcome of the work presented in this thesis. 


\section{References}

1

HeCaToS project (Hepatic and Cardiac Toxicity Systems modelling). (2014).

Verheijen, M., Krauskopf, J., Kleinjans, J. C., de Kok, T. M. \& Caiment, F. Development and regulatory application of microRNA biomarkers. Biomark Med 9, 1137-1151, doi:10.2217/bmm.15.50 (2015). Bernstein, D. Anthracycline Cardiotoxicity Worrisome Enough to Have You Quaking? Circ Res 122, 188190, doi:10.1161/Circresaha.117.312395 (2018).

Fernandez - Chas, M., Curtis, M. \& Niederer, S. Mechanism of doxorubicin cardiotoxicity evaluated by integrating multiple molecular effects into a biophysical model. British journal of pharmacology 175, 763-781 (2018).

Arcamone, F., Cassinelli, G., Di Marco, A. \& Gaetani, M. Patent application Farmitalia Research Laboratories. NSA 251 (1967).

Dobson, J. M., Hohenhaus, A. E. \& Peaston, A. E. Cancer chemotherapy. Small Animal Clinical Pharmacology 2nd ed.(Maddison, JE, Page, SW and Church, DB eds.), Saunders Elsevier, Edinburgh, 330366 (2008).

McGowan, J. V. et al. Anthracycline Chemotherapy and Cardiotoxicity. Cardiovasc Drug Ther 31, 63-75, doi:10.1007/s10557-016-6711-0 (2017).

\section{Menna, P. \& Salvatorelli, E. Primary Prevention Strategies for Anthracycline Cardiotoxicity: A Brief} Overview. Chemotherapy 62, 159-168, doi:10.1159/000455823 (2017).

Kim, S. et al. PubChem substance and compound databases. Nucleic Acids Res 44, D1202-D1213 (2015). Burger, M. C. ChemDoodle Web Components: HTML5 toolkit for chemical graphics, interfaces, and informatics. Journal of cheminformatics 7, 35 (2015).

Cappetta, D. et al. SIRT1 activation attenuates diastolic dysfunction by reducing cardiac fibrosis in a model of anthracycline cardiomyopathy. Int J Cardiol 205, 99-110, doi:10.1016/j.ijcard.2015.12.008 (2016).

Raj, S., Franco, V. I. \& Lipshultz, S. E. Anthracycline-induced cardiotoxicity: a review of pathophysiology, diagnosis, and treatment. Curr Treat Options Cardiovasc Med 16, 315, doi:10.1007/s11936-014-0315-4 (2014).

Zhang, Y. W., Shi, J. J., Li, Y. J. \& Wei, L. Cardiomyocyte death in doxorubicin-induced cardiotoxicity. Arch Immunol Ther Ex 57, 435-445, doi:10.1007/s00005-009-0051-8 (2009).

Chatterjee, K., Zhang, J. Q., Honbo, N. \& Karliner, J. S. Doxorubicin Cardiomyopathy. Cardiology 115, 155-162, doi:10.1159/000265166 (2010).

Chen, B., Peng, X. Y., Pentassuglia, L., Lim, C. C. \& Sawyer, D. B. Molecular and cellular mechanisms of anthracycline cardiotoxicity. Cardiovasc Toxicol 7, 114-121, doi:10.1007/s12012-007-0005-5 (2007). Carvalho, F. S. et al. Doxorubicin-Induced Cardiotoxicity: From Bioenergetic Failure and Cell Death to Cardiomyopathy. Med Res Rev 34, 106-135, doi:10.1002/med.21280 (2014).

Kumar, S., Marfatia, R., Tannenbaum, S., Yang, C. \& Avelar, E. Doxorubicin-induced cardiomyopathy 17 years after chemotherapy. Tex Heart Inst J 39, 424-427 (2012).

Takemura, G. \& Fujiwara, H. Doxorubicin-induced cardiomyopathy from the cardiotoxic mechanisms to management. Prog Cardiovasc Dis 49, 330-352, doi:10.1016/j.pcad.2006.10.002 (2007).

Yang, F., Teves, S. S., Kemp, C. J. \& Henikoff, S. Doxorubicin, DNA torsion, and chromatin dynamics. Biochim Biophys Acta 1845, 84-89, doi:10.1016/j.bbcan.2013.12.002 (2014).

Edwardson, D. W. et al. Role of Drug Metabolism in the Cytotoxicity and Clinical Efficacy of Anthracyclines. Curr Drug Metab 16, 412-426 (2015).

Damiani, R. M. et al. Pathways of cardiac toxicity: comparison between chemotherapeutic drugs doxorubicin and mitoxantrone. Arch Toxicol 90, 2063-2076, doi:10.1007/s00204-016-1759-y (2016). Sorensen, J. C. et al. Mitochondria: Inadvertent targets in chemotherapy-induced skeletal muscle toxicity and wasting? Cancer Chemother Pharmacol 78, 673-683, doi:10.1007/s00280-016-3045-3 (2016). Burridge, P. W. et al. Human induced pluripotent stem cell-derived cardiomyocytes recapitulate the predilection of breast cancer patients to doxorubicin-induced cardiotoxicity. Nat Med 22, 547-556, doi:10.1038/nm.4087 (2016). Berthiaume, J. M. \& Wallace, K. B. Adriamycin-induced oxidative mitochondrial cardiotoxicity. Cell Biol Toxicol 23, 15-25, doi:10.1007/s10565-006-0140-y (2007).

Varga, Z. V., Ferdinandy, P., Liaudet, L. \& Pacher, P. Drug-induced mitochondrial dysfunction and cardiotoxicity. Am J Physiol Heart Circ Physiol 309, H1453-1467, doi:10.1152/ajpheart.00554.2015 (2015).

Verheijen, M. et al. Bringing in vitro analysis closer to in vivo: Studying doxorubicin toxicity and associated mechanisms in 3D human microtissues with PBPK-based dose modelling. Toxicol Lett 294, 184-192, doi:10.1016/j.toxlet.2018.05.029 (2018). 
Verheijen, M., Krauskopf, J., Kleinjans, J. C., de Kok, T. M. \& Caiment, F. Development and regulatory application of microRNA biomarkers. Biomark Med 9, 1137-1151, doi:10.2217/bmm.15.50 (2015). Olson, H. et al. Concordance of the toxicity of pharmaceuticals in humans and in animals. Regul Toxicol Pharm 32, 56-67, doi:10.1006/rtph.2000.1399 (2000). Elliott, N. T. \& Yuan, F. A review of three-dimensional in vitro tissue models for drug discovery and transport studies. J Pharm Sci 100, 59-74, doi:10.1002/jps.22257 (2011). Achilli, T. M., Meyer, J. \& Morgan, J. R. Advances in the formation, use and understanding of multicellular spheroids. Expert Opin Biol Th 12, 1347-1360, doi:10.1517/14712598.2012.707181 (2012). Zuppinger, C. 3D culture for cardiac cells. Bba-Mol Cell Res 1863, 1873-1881, doi:10.1016/j.bbamcr.2015.11.036 (2016). Kuepfer, L. et al. A model-based assay design to reproduce in vivo patterns of acute drug-induced toxicity. Arch Toxicol, 1-3 (2017). Eksborg, S., Strandler, H.-S., Edsmyr, F., Näslund, I. \& Tahvanainen, P. Pharmacokinetic study of IV infusions of adriamycin. European Journal of Clinical Pharmacology 28, 205-212, doi:10.1007/bf00609693 (1985).

Crick, F. Central dogma of molecular biology. Nature 227, 561-563 (1970)

Chen, Z. J. et al. Fresh versus Frozen Embryos for Infertility in the Polycystic Ovary Syndrome. New Engl J Med 375, 523-533, doi:10.1056/NEJMoa1513873 (2016).

Payne, S. H. The utility of protein and mRNA correlation. Trends Biochem Sci 40, 1-3, doi:10.1016/j.tibs.2014.10.010 (2015).

Ghazalpour, A. et al. Comparative Analysis of Proteome and Transcriptome Variation in Mouse. Plos Genet 7, doi:ARTN e1001393 10.1371/journal.pgen.1001393 (2011).

Shen, X., Young, R., Canty, J. M. \& Qu, J. Quantitative proteomics in cardiovascular research: global and targeted strategies. Proteomics Clin App/ 8, 488-505, doi:10.1002/prca.201400014 (2014).

Millioni, R. et al. High Abundance Proteins Depletion vs Low Abundance Proteins Enrichment: Comparison of Methods to Reduce the Plasma Proteome Complexity. Plos One 6, doi:ARTN e19603 10.1371/journal.pone.0019603 (2011).

Webb-Robertson, B. J. et al. Review, evaluation, and discussion of the challenges of missing value imputation for mass spectrometry-based label-free global proteomics. J Proteome Res 14, 1993-2001, doi:10.1021/pr501138h (2015).

Gry, M. et al. Correlations between RNA and protein expression profiles in 23 human cell lines. BMC genomics 10, doi:Artn 365 10.1186/1471-2164-10-365 (2009).

Vogel, C. \& Marcotte, E. M. Insights into the regulation of protein abundance from proteomic and transcriptomic analyses. Nature Reviews Genetics 13, 227-232, doi:10.1038/nrg3185 (2012).

Maier, T., Guell, M. \& Serrano, L. Correlation of mRNA and protein in complex biological samples. Febs Lett 583, 3966-3973, doi:10.1016/j.febslet.2009.10.036 (2009).

Oliveto, S., Mancino, M., Manfrini, N. \& Biffo, S. Role of microRNAs in translation regulation and cancer. World J Biol Chem 8, 45-56, doi:10.4331/wjbc.v8.i1.45 (2017).

van den Hoogenhof, M. M. G., Pinto, Y. M. \& Creemers, E. E. RNA Splicing Regulation and Dysregulation in the Heart. Circ Res 118, 454-468, doi:10.1161/Circresaha.115.307872 (2016).

Richard, H. et al. Prediction of alternative isoforms from exon expression levels in RNA-Seq experiments. Nucleic Acids Res 38, doi:ARTN e112 10.1093/nar/gkq041 (2010).

Wang, E. T. et al. Alternative isoform regulation in human tissue transcriptomes. Nature 456, 470-476, doi:10.1038/nature07509 (2008).

Aonuma, T., Bayoumi, A. S., Tang, Y. \& Kim, I.-m. A circular RNA regulator quaking: a novel gold mine to be unfolded in doxorubicin-mediated cardiotoxicity. Non-coding RNA Investigation (2018).

Greene, J. et al. Circular RNAs: Biogenesis, Function and Role in Human Diseases. Front Mol Biosci 4, 38, doi:10.3389/fmolb.2017.00038 (2017).

Kaikkonen, M. U., Lam, M. T. \& Glass, C. K. Non-coding RNAs as regulators of gene expression and epigenetics. Cardiovasc Res 90, 430-440, doi:10.1093/cvr/cvr097 (2011).

Liu, J. Control of protein synthesis and
doi:10.1016/j.ceb.2008.01.006 (2008). Nilsen, T. W. Mechanisms of microRNA-mediated gene regulation in animal cells. Trends Genet 23, 243249, doi:10.1016/j.tig.2007.02.011 (2007).

4 Huntzinger, E. \& Izaurralde, E. Gene silencing by microRNAs: contributions of translational repression and mRNA decay. Nature reviews. Genetics 12, 99-110, doi:10.1038/nrg2936 (2011). $\mathrm{Wu}, \mathrm{L}$. \& Belasco, J. G. Let me count the ways: mechanisms of gene regulation by miRNAs and siRNAs. Mol Cell 29, 1-7, doi:10.1016/j.molcel.2007.12.010 (2008). 
Tang, L. et al. microRNA inhibitors: Natural and artificial sequestration of microRNA. Cancer Lett 407, 139-147, doi:10.1016/j.canlet.2017.05.025 (2017).

Iorio, M. V., Piovan, C. \& Croce, C. M. Interplay between microRNAs and the epigenetic machinery: an intricate network. Biochim Biophys Acta 1799, 694-701, doi:10.1016/j.bbagrm.2010.05.005 (2010).

Memczak, S. et al. Circular RNAs are a large class of animal RNAs with regulatory potency. Nature 495, 333-338, doi:10.1038/nature11928 (2013).

Huang, Y. et al. Molecular functions of small regulatory noncoding RNA. Biochemistry (Mosc) 78, 221 230, doi:10.1134/S0006297913030024 (2013).

Carthew, R. W. \& Sontheimer, E. J. Origins and Mechanisms of miRNAs and siRNAs. Cell 136, 642-655, doi:10.1016/j.cell.2009.01.035 (2009).

Lewis, B. P., Burge, C. B. \& Bartel, D. P. Conserved seed pairing, often flanked by adenosines, indicates that thousands of human genes are microRNA targets. Cell 120, 15-20 (2005).

Panda, A. C., Grammatikakis, I., Munk, R., Gorospe, M. \& Abdelmohsen, K. Emerging roles and context of circular RNAs. Wiley Interdiscip Rev RNA 8, doi:10.1002/wrna.1386 (2017).

Ebbesen, K. K., Kjems, J. \& Hansen, T. B. Circular RNAs: Identification, biogenesis and function. Bba-Gene Regul Mech 1859, 163-168, doi:10.1016/j.bbagrm.2015.07.007 (2016).

Rong, D. et al. An emerging function of circRNA-miRNAs-mRNA axis in human diseases. Oncotarget, doi:10.18632/oncotarget.19154 (2017).

Qu, S. B. et al. Circular RNA: A new star of noncoding RNAs. Cancer Lett 365, 141-148, doi:10.1016/j.canlet.2015.06.003 (2015).

Cortes-Lopez, M. \& Miura, P. Emerging Functions of Circular RNAs. Yale J Biol Med 89, 527-537 (2016). Liu, J., Liu, T., Wang, X. \& He, A. Circles reshaping the RNA world: from waste to treasure. Mol Cancer 16, 58, doi:10.1186/s12943-017-0630-y (2017).

Sanger, H. L., Klotz, G., Riesner, D., Gross, H. J. \& Kleinschmidt, A. K. Viroids are single-stranded covalently closed circular RNA molecules existing as highly base-paired rod-like structures. Proceedings of the National Academy of Sciences 73, 3852-3856 (1976).

Salzman, J., Gawad, C., Wang, P. L., Lacayo, N. \& Brown, P. O. Circular RNAs Are the Predominant Transcript Isoform from Hundreds of Human Genes in Diverse Cell Types. Plos One 7, doi:ARTN e30733 10.1371/journal.pone.0030733 (2012).

Hansen, T. B. et al. Natural RNA circles function as efficient microRNA sponges. Nature 495, 384-388, doi:10.1038/nature11993 (2013).

Russo, V. E., Martienssen, R. A. \& Riggs, A. D. Epigenetic mechanisms of gene regulation. (Cold Spring Harbor Laboratory Press, 1996).

Felsenfeld, G. A Brief History of Epigenetics. Csh Perspect Biol 6, doi:ARTN a018200 10.1101/cshperspect.a018200 (2014).

Jin, B., Li, Y. \& Robertson, K. D. DNA methylation: superior or subordinate in the epigenetic hierarchy? Genes \& cancer 2, 607-617 (2011).

Senner, C. E. The role of DNA methylation in mammalian development. Reprod Biomed Online 22, 529535, doi:10.1016/j.rbmo.2011.02.016 (2011).

Gayon, J. From Mendel to epigenetics: History of genetics. Cr Biol 339, 225-230,

doi:10.1016/j.crvi.2016.05.009 (2016). 


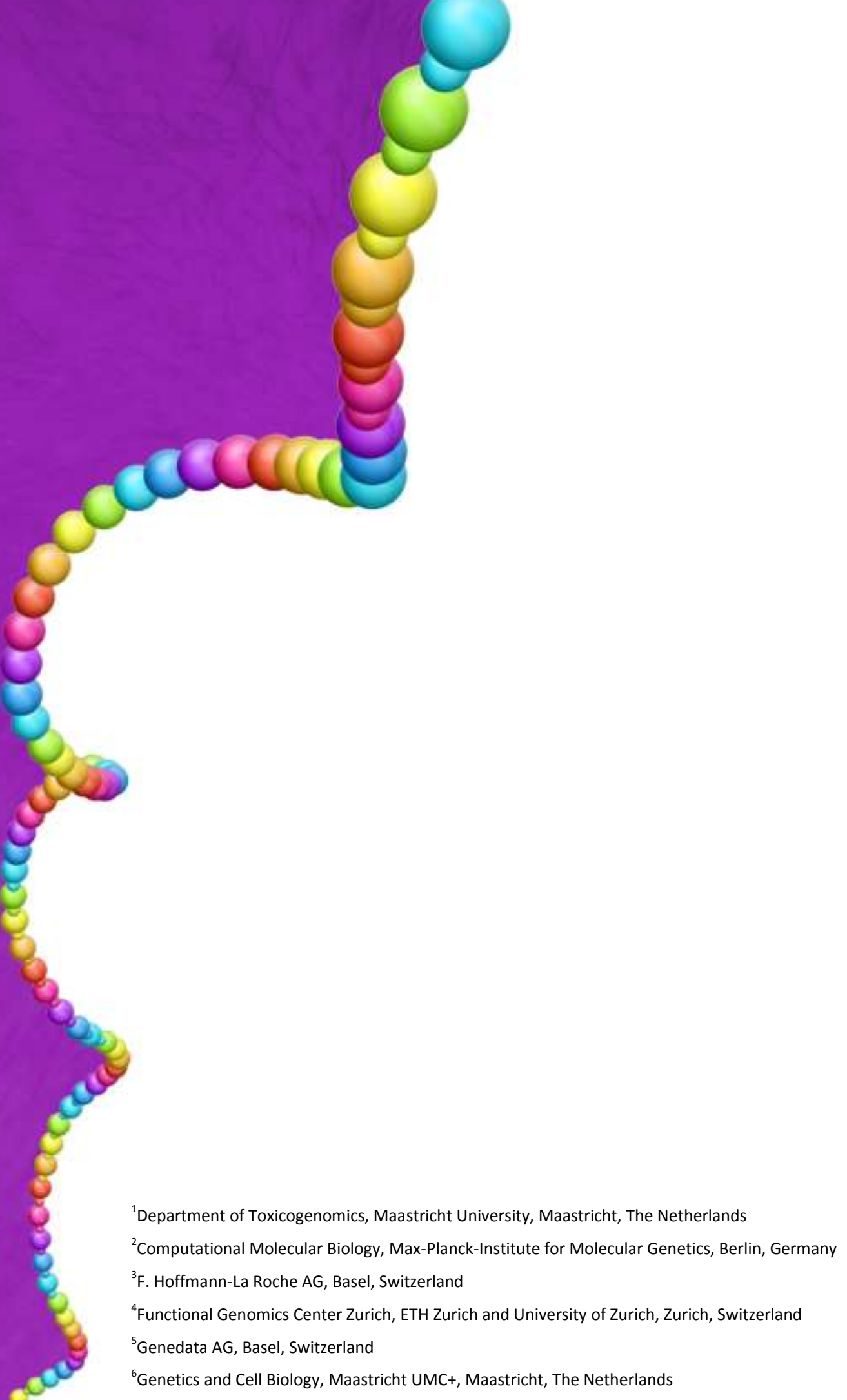




\section{Chapter 2}

\section{DMSO induces drastic changes in}

human cellular processes and

\section{epigenetic landscape în vitro}

Marcha C.T. Verheijen ${ }^{1}$

M. Lienhard ${ }^{2}$

Y. Schrooders ${ }^{1}$

O. Clayton ${ }^{3}$

R. Nudischer ${ }^{3}$

S. Boerno ${ }^{2}$

B. Timmermann ${ }^{2}$

N. Selevsek ${ }^{4}$

R. Schlapbach ${ }^{4}$

H. Gmuender ${ }^{5}$

S. Gotta ${ }^{5}$

J. Geraedts ${ }^{6}$

R. Herwig ${ }^{2}$

J. Kleinjans ${ }^{1}$

F. Caiment ${ }^{1}$

Published:

Scientific Reports, 2019

Volume 9 (1), Page 4641 


\section{Abstract}

Though clinical trials for medical applications of dimethyl sulfoxide (DMSO) reported toxicity in the 1960s, later, the FDA classified DMSO in the safest solvent category. DMSO became widely used in many biomedical fields and biological effects were overlooked. Meanwhile, biomedical science has evolved towards sensitive highthroughput techniques and new research areas, including epigenomics and microRNAs. Considering its wide use, especially for cryopreservation and in vitro assays, we evaluated biological effect of DMSO using these technological innovations. We exposed 3D cardiac and hepatic microtissues to medium with or without $0.1 \%$ DMSO and analyzed the transcriptome, proteome and DNA methylation profiles. In both tissue types, transcriptome analysis detected $>2000$ differentially expressed genes affecting similar biological processes, thereby indicating consistent cross-organ actions of DMSO. Furthermore, microRNA analysis revealed large-scale deregulations of cardiac microRNAs and smaller, though still massive, effects in hepatic microtissues. Genome-wide methylation patterns also revealed tissue-specificity. While hepatic microtissues demonstrated non-significant changes, findings from cardiac microtissues suggested disruption of DNA methylation mechanisms leading to genome-wide changes. The extreme changes in microRNAs and alterations in the epigenetic landscape indicate that DMSO is not inert. Its use should be reconsidered, especially for cryopreservation of embryos and oocytes, since it may impact embryonic development. 


\section{Introduction}

Dimethyl sulfoxide (DMSO) is an organic polar aprotic molecule with an amphipathic nature that is ideal for dissolving poorly soluble polar and non-polar molecules. DMSO is widely used as solvent in toxicology and pharmacology, for cryopreservation of cells, and as penetration enhancer during topological treatments. The use of DMSO is so obvious that applied concentrations are often unreported. DMSO is generally accepted as nontoxic below $10 \%(\mathrm{v} / \mathrm{v})$ and, in practice, it is assumed that effects of DMSO are negligible $e^{[1-3]}$.

Research from the 1960's till 1990's pinpointed a lot of biologically relevant effects of DMSO. It was even actively investigated for medical use, but because of adverse effects especially affecting the eyes, most clinical trials were halted in 1965 by the United States Food and Drug Administration (FDA). At this point in time, DMSO was seen as extremely toxic, comparable to thalidomide ${ }^{[4,5]}$. The effects of DMSO differ depending on dose and route of administration. Introducing DMSO concentrations higher than $50 \%$ into the blood resulted in instant hemolysis, white cell stacking and fibrinogen precipitation while direct injection of DMSO intravenously could cause local irritation and necrosis ${ }^{[6]}$. LD50 values gained from monkeys indicated that 880 grams applied on the skin or 320 grams injected intravenously would result in 50\% mortality in $80 \mathrm{~kg}$ humans ${ }^{[7]}$. This relatively high dose changed the view on DMSO toxicity and the FDA classified DMSO in the same class as ethanol, namely class 3 solvent, which is the safest category with low toxic potential at levels normally accepted in pharmaceuticals $^{[8]}$. This made the wide use of DMSO possible.

While generally used at relatively low concentrations, DMSO still has medically useful properties such as inducing anti-inflammation, nerve blockage (analgesia), diuretics, vasodilation and muscle relaxation ${ }^{[9]}$. Furthermore, in cell biology, DMSO is also used as inducer of cell differentiation, free radical scavenger and radioprotectant, but most often for cryopreservation. Cell cultures for research are often stored in liquid nitrogen using slow cooling methodology. To prevent damage by intracellular ice crystals, cells are slowly cooled to -80 degrees in the presence of $10 \%$ DMSO before storage in liquid nitrogen ${ }^{[3]}$. However, this procedure is insufficient for biomedical 
applications. Because slow cooling still induces damage due to extracellular ice crystals, cryopreservation of human oocytes and embryos for in vitro fertilization (IVF) is accomplished by vitrification, in which higher concentrations of cryoprotectants are used to prevent ice formation not only in the cells, but in the entire solution. Higher concentrations are achieved by using mixtures (for example 15\% DMSO in combination with $15 \%$ ethylene glycol) which reduces the amount and toxicity of the individual cryoprotectants $^{[10]}$. Furthermore, DMSO readily crosses most tissue membranes of lower animals and $\operatorname{man}^{[9]}$. For example, 2 hours after topological application, DMSO had penetrated all investigated hard and soft tissues (6 and 15 resp.) in rats ${ }^{[11]}$. DMSO is also able to enhance the permeability of other low molecular weight compounds, thereby making them pass membranes or going deeper into a tissue as they normally would $^{[12]}$, a property highly useful in topological therapies. In 2009, the first to obtain FDA approval for topological DMSO usage was PENNSAID ${ }^{\circledR}$, which contains diclofenac in a carrier with $45.5 \%$ DMSO $^{[13,14]}$. This relatively high concentration of DMSO in topological applications is necessary because the skin is harder to penetrate than cell membranes.

Most insights into the molecular effects of DMSO were obtained last century often using high doses. In the meantime, biomedical science has evolved towards more sensitive high-throughput techniques and towards new areas of research, including epigenome modifications and microRNA-mediated gene silencing. Considering its wide use in many biological fields, we analyzed a relatively low dose of DMSO $(0.1 \%$, which is commonly applied in cell assays) to study the impact on the proteome, transcriptome and the epigenome. For this, we exposed in vitro 3D microtissues (a maturing iPSC-derived cardiac model and a mature hepatic model) to $0.1 \%$ DMSO and we collected samples in triplicate at 7 different time points during 2 weeks exposure (2h, 8h, 24h, 72h, 168h, 240h and 336h). Thereafter, DMSO effects were assessed with full transcriptome analysis (using ribo-depleted total RNA sequencing and microRNA sequencing), whole-genome methylation profiling (using MeDIP-seq) and proteomics analysis (using mass spectrometry). Our analysis clearly demonstrated that DMSO cannot be considered biologically inert but induces large alterations in microRNAs (miRNA) and epigenetic landscape, especially in the maturing cardiac model. 


\section{Methods}

\section{Samples}

In this study, 3D InSight ${ }^{\mathrm{TM}}$ Human Cardiac Microtissues (InSphero, for beta-testers) were used, containing a co-culture of approximately 4000 iPSC-derived human cardiomyocytes from a female Caucasian donor with no known disease phenotype and 1000 cardiac fibroblasts from an 18 year old Caucasian male. The MTs were cultured in $3 \mathrm{D}$ Insight ${ }^{\mathrm{TM}}$ Human Cardiac Microtissue Maintenance Medium (InSphero). Furthermore, 3D InSight ${ }^{\mathrm{TM}}$ Human Liver Microtissues (InSphero) were used, approximately 1000 primary human hepatocytes (multi-donor pool of 5 males and 5 females between 7-59 years old) in co-culture with 1000 primary human Kupffer cells from a Caucasian 27 year old of unreported gender. The MTs were cultured in 3D Insight $^{\mathrm{TM}}$ Human Liver Microtissue Maintenance Medium- AF (InSphero).

\section{Exposure}

The microtissues (MTs) were exposed to medium only (= untreated) or medium containing $0.1 \%$ DMSO for two weeks. Medium was changed three times daily, in concordance with the experimental design of our current research ${ }^{[15]}$. During the 2 week exposure, there were a total of 7 sampling time points at 2, 8, 24, 72, 168, 240 and 336 hours, which were sampled in triplicate (total 21 samples per condition). Per sample, 36 cardiac MTs or 54 hepatic MTs were used in order to obtain sufficient amounts of RNA for sequencing.

\section{RNA sequencing sample preparation}

Total RNA was isolated using the AllPrep DNA/RNA/miRNA Universal Kit (Qiagen). The sample was depleted of ribosomal RNA using the Ribo-Zero Gold rRNA Removal kit (Human/Mouse/Rat) (Illumina ${ }^{\circledR}$ ) and prepared for sequencing with the SENSE total RNA library preparation kit (Lexogen). After library preparation, the quality was assessed on Agilent 4200 TapeStation and library concentration was determined by Qubit $^{\mathrm{TM}}$ before sequencing (paired-end 100bp) on the HiSeq2000. Two files per sample were obtained, split in left and right reads, and Lexogen adapter sequences (first 12 bases of all reads) were removed using Trimmomatic ${ }^{[16]}$ (v.0.33). The quality of the 
sequencing data was checked using FastQ $C^{[17]}$ (v.0.10.1) before and after trimming. For cardiac samples, no samples were discarded because of poor quality. Two samples were discarded because of low read counts (UNTR_002_3 and UNTR_240_3) and principal component analysis (Supplementary Figure 1) revealed 4 outliers which were removed (UNTR_008_3, UNTR_168_2, UNTR_240_2 and DMSO_336_2). Finally, because only one replicate of 240 hour untreated samples remained, all samples of this time point were removed from statistical analysis. For hepatic samples, no samples were discarded.

\section{RNA data-analysis}

Read counts for genes were obtained by aligning the reads to the reference genome (Genome Reference Consortium Human Build 38, GRCh38.p7) using RSEM ${ }^{[18]}$ (v.1.2.28) with the paired-end and Bowtie $2^{[19]}$ (v.2.2.6) option. Thereafter, the read counts for each gene were used for determination of differentially expressed genes using the DESeq2 ${ }^{[20]} R$ package (v.1.16.11). Here, the design was set according to exposure (UNTR or DMSO), other settings were kept to their default parameters, except for minimum count, which was set to an average read count of one across all samples. Finally, remaining ribosomal genes were filtered out of the dataset to ensure complete ribosomal depletion of the data.

\section{MiRNA sequencing sample preparation}

An aliquot of the isolated total RNA was selected on size and ligated using the TruSeq Small RNA Library Prep Kit (Illumina ${ }^{\circledR}$ ). After library preparation, the samples were sequenced (paired-end 100bp) on the HiSeq2000. Adapter sequences were trimmed ${ }^{[21]}$ and reads between 16 and 35bp were kept for analysis. Three samples were discarded because of low read counts (Cardiac: UNTR_168_1, UNTR_168_2 and Hepatic: DMSO_002_3). Furthermore, principal component analysis (supplementary figure 1) revealed no outliers. 


\section{MiRNA data-analysis}

MiRNA data analysis was done similarly as described previously ${ }^{[22]}$, except for the use of mirDeep2, which does not gain additional information when using human samples. In short, PatMaN ${ }^{[23]}$ (v.1.2.2) was used to align trimmed reads to the human genome without allowing mismatches or gaps. To obtain complete read counts for 3' and $5^{\prime}$ miRNA species, the mapping output was parsed.

\section{Proteomics}

Proteomics data was also obtained and analyzed. Methods and results are included in supplementary data.

\section{MeDIP sequencing}

For preparation of MeDIP-Seq libraries, the low input MeDIP protocol ${ }^{[24]}$ was modified. DNA was fragmented to $100-200 \mathrm{bp}$ using the Covaris S2 system. Because of lower DNA yield for cardiac 0.1\% DMSO samples, the triplicates were pooled before fragmentation. End repair and A tailing was performed using the NEBNext ${ }^{\circledR}$ Ultra $^{\text {TM }}$ library prep kit for Illumina ${ }^{\circledR}$ (NEB), adapters were ligated with NEBNext ${ }^{\circledR}$ Ultra $^{\text {TM }}$ Ligation Module (NEB) and samples were purified using Agencourt ${ }^{\circledR}$ AMPure ${ }^{\circledR}$ XP beads (Beckman Coulter). Methylated fragments were captured using the MagMeDIP kit (Diagenode). In short, denatured DNA was mixed with anti-5-meC-antibody and captured using magnetic beads. Capture efficiency was determined by qPCR against spiked-in Lambda-DNA fragments in precapture and postcapture library samples. Libraries were amplified in a final PCR step using barcoded TruSeq primers. Quality was assessed on Agilent Bioanalyzer 2100 and library concentration was determined by Qubit $^{\mathrm{TM}}$ and qPCR.

\section{MeDIP data-analysis}

In order to gain exhaustive genome-wide coverage the triplicate samples that have been sequenced individually were merged before alignment. MeDIP sequencing reads were aligned to the GRCh38 reference genome using bwa Version 0.7.15-r $1140^{[25]}$, and analyzed in 250 base windows using the R/bioconductor package QSEA ${ }^{[26]}$ (v.1.4.0) with standard parameters. Within QSEA, the MeDIP enrichment was calibrated with 
450k methylation array measurements of primary hepatocytes (GSM999339) and cardiac myocytes (HCM, GSM999381) from ENCODE ${ }^{[27]}$, for the hepatic and cardiac micro tissues respectively. To this end, beta values of the calibration samples were computed with the R/bioconductor package Minfi ${ }^{[28]}$ (v.1.24.0), genomic locations of the array probes were mapped from GRCh37 to GRCh38 using the UCSC liftOver command line tool ${ }^{[29]}$, and probes within 250 base windows were averaged.

Differentially methylated regions obtained from QSEA were annotated with gene, exon, and promoter (transcription start site +/- 2 kilobases) information from RefSeq, ENCODE TFBS and model based CPG islands, all obtained via the UCSC table browser. Since ENCODE TFBS were not available for GRCh38, genomic locations were mapped from GRCh37 using the liftOver tool. 


\section{Results}

Human 3D microtissues (MTs) of a maturing cardiac model and a mature hepatic model were exposed to culture medium with or without $0.1 \%$ DMSO for two weeks with sampling time points at 2, 8, 72, 168, 240 and 336 hours. The proteome (approximately 2,000 measured proteins), the full transcriptome (including miRNAs) and whole-genome methylation were measured on material obtained from the same sample. Figure 1 contains a graphical overview of the experimental design. In order to obtain a first overview of DMSO-induced cross-omics effects, amounts of differentially changed entities (all corrected for multiple testing using FDR $<0.05$ ) are summarized for each platform. Numbers of differentially changed entities differed between the tissue types, with cardiac samples showing a larger effect of DMSO than hepatic, with the exception of mRNAs. This difference is especially noticeable for miRNAs and genome methylation. Because proteomics data was least informative due to its partial nature, these results were included in supplementary data. Furthermore, principal component analysis (PCA), using averages of triplicates, for each platform (Figure 2 \& supplementary data) depicts clear differences between 0.1\% DMSO exposed (DMSO) and untreated (UNTR) samples, with the exception of methylation in hepatic MTs.
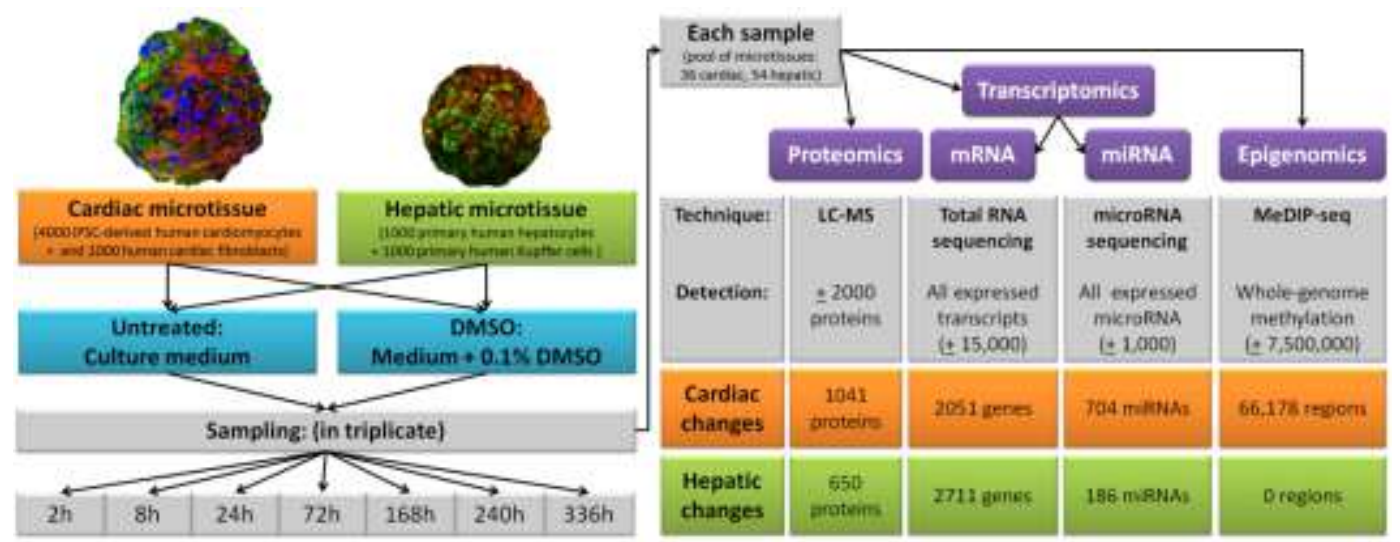

Figure 1: Graphical overview of experimental design combined with summary of differential entities of each analysis method. Tissue-specific information is depicted in orange for cardiac and green for hepatic. Furthermore, exposures are coloured blue and measurement platforms purple. Abbreviations: $h=$ hours; $m R N A=$ messenger RNA ; $m i R N A=$ microRNA 
a)

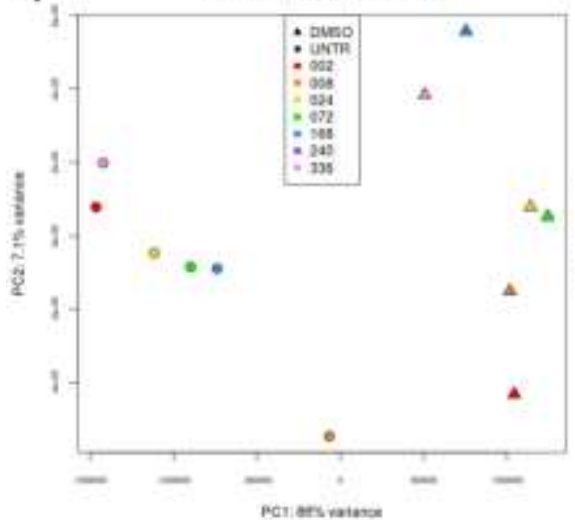

b)

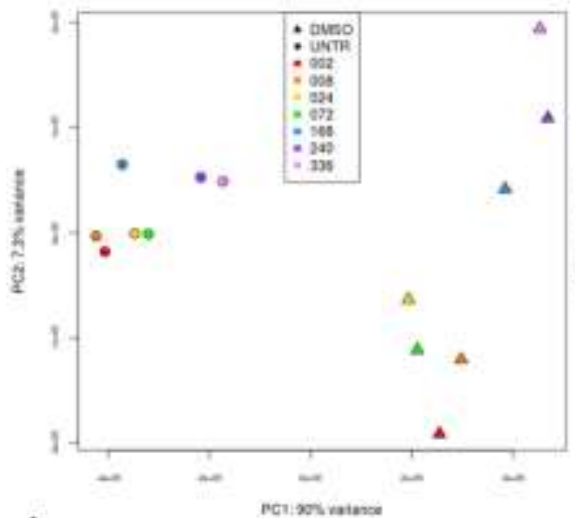

c)

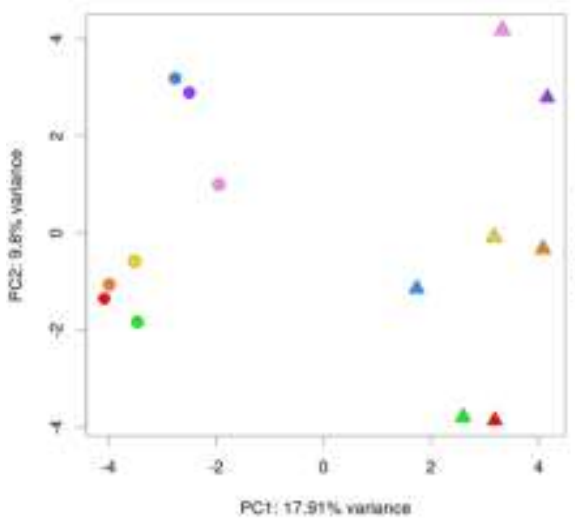

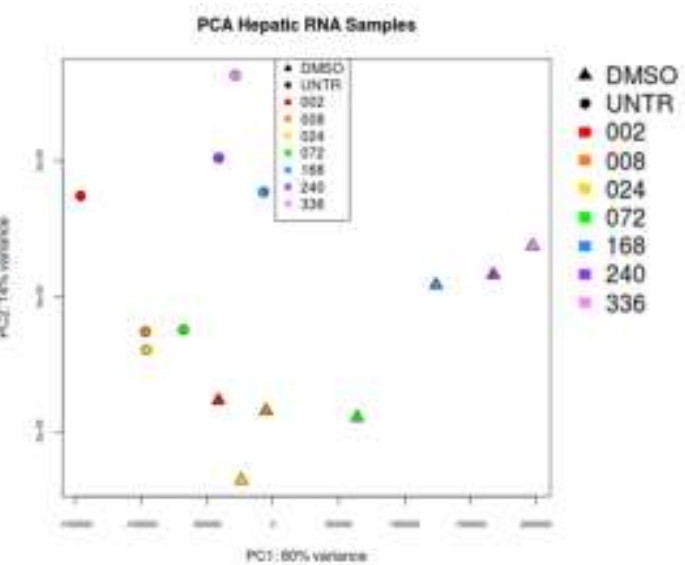

PCA Hepatio miRNA Samplas

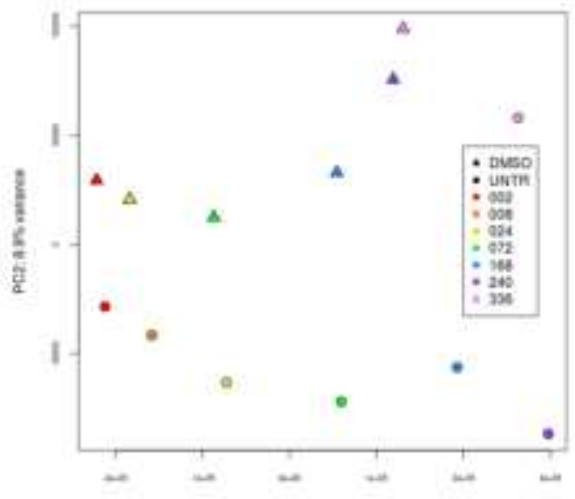

PCA hepatic DNA methylation

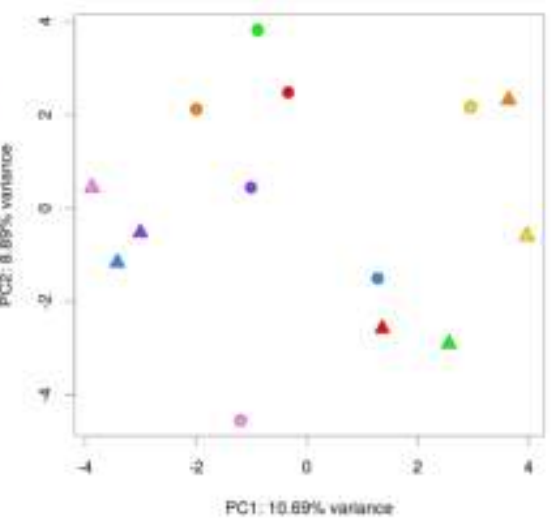

Figure 2: PCAs depicting differences between DMSO and UNTR for all measured platforms.

a) PCA of RNAs indicates clear differences in RNA expression between DMSO (triangle) and UNTR (circles). Cardiac samples (left) are more distinct from UNTR than hepatic samples (right). b) PCA of miRNAs reveals clear separation between DMSO and UNTR in cardiac samples, while hepatic samples seem more susceptible to the duration of the exposure (as seen by colour pattern that corresponds to the specific time points, see legend). c) PCA of promotor methylation indicates differences between DMSO and UNTR for cardiac samples but not for hepatic samples 
To study the molecular effects of DMSO, affected cellular processes were analysed using the full transcriptome. Thereafter, tissue-specific effects of DMSO on regulation of gene expression were investigated by analysing changes in miRNAs and genomemethylation.

\section{DMSO effects on cellular processes}

DMSO effects on mRNAs were depicted by of PCA (Figure 2 A-B). The clear separation between UNTR and 0.1\% DMSO indicated that DMSO was able to affect cellular processes by altering gene expression. Comparison between DMSO and UNTR resulted in 2051 differentially expressed genes (DEGs = FDR <0.05; of which 871 with $|\log 2 \mathrm{FC}|>1$ ) in cardiac MTs and 2711 DEGs (of which 1879 with $|\log 2 \mathrm{FC}|>1$ ) in hepatic MTs, of which $60.7 \%$ and $62.9 \%$ DEGs were downregulated respectively.

\section{Pathway analysis of DEGs}

To identify cellular processes affected by DMSO exposure, DEGs were used for pathway overrepresentation analysis using ConsensusPathDB ${ }^{[30]}$ with the curated Reactome database $^{[31]}$. Significantly overrepresented pathways (q-value<0.05) were ordered using the hierarchical connections between (sub-) pathways obtained from the Reactome Pathway Browser (supplementary Tables 1\&2). A summary containing the highest hierarchical pathway levels (from now on referred to as clusters) is included in Table 1 for cardiac and Table 2 for hepatic MTs.

Through pathway analysis on DEGs, 225 significantly overrepresented pathways (qvalue $<0.05$ ) were found in cardiac MTs, which corresponded to 19 clusters (out of a total of 25 clusters in the Pathway Browser), and 167 pathways corresponding to 16 clusters in hepatic MTs. There was substantial overlap between the tissue types, with 60 pathways and 15 clusters found in both. Although there were differences in magnitude of DMSO effect between tissue types, the affected biological processes by DMSO do not appear to be tissue-specific. 


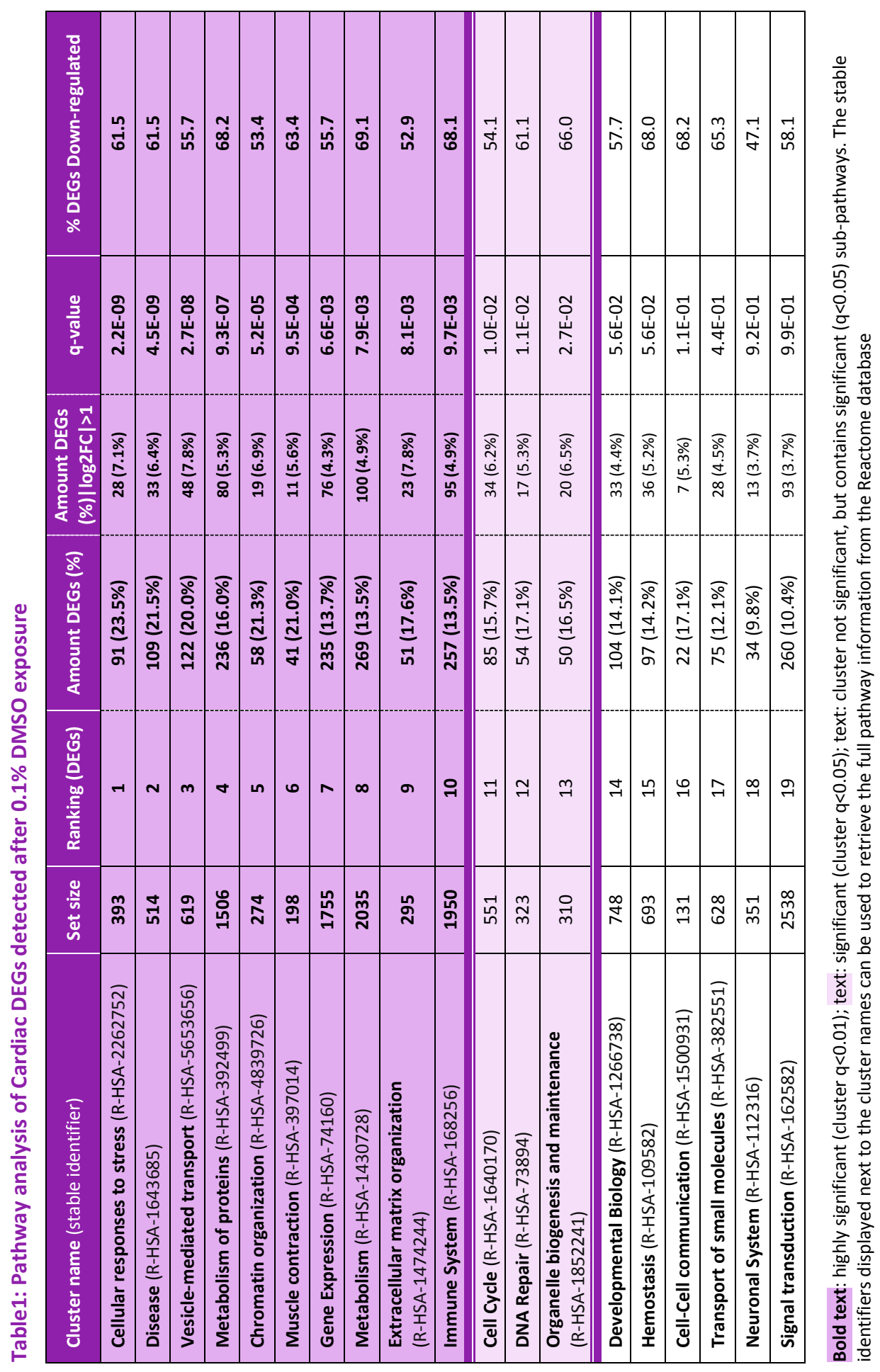




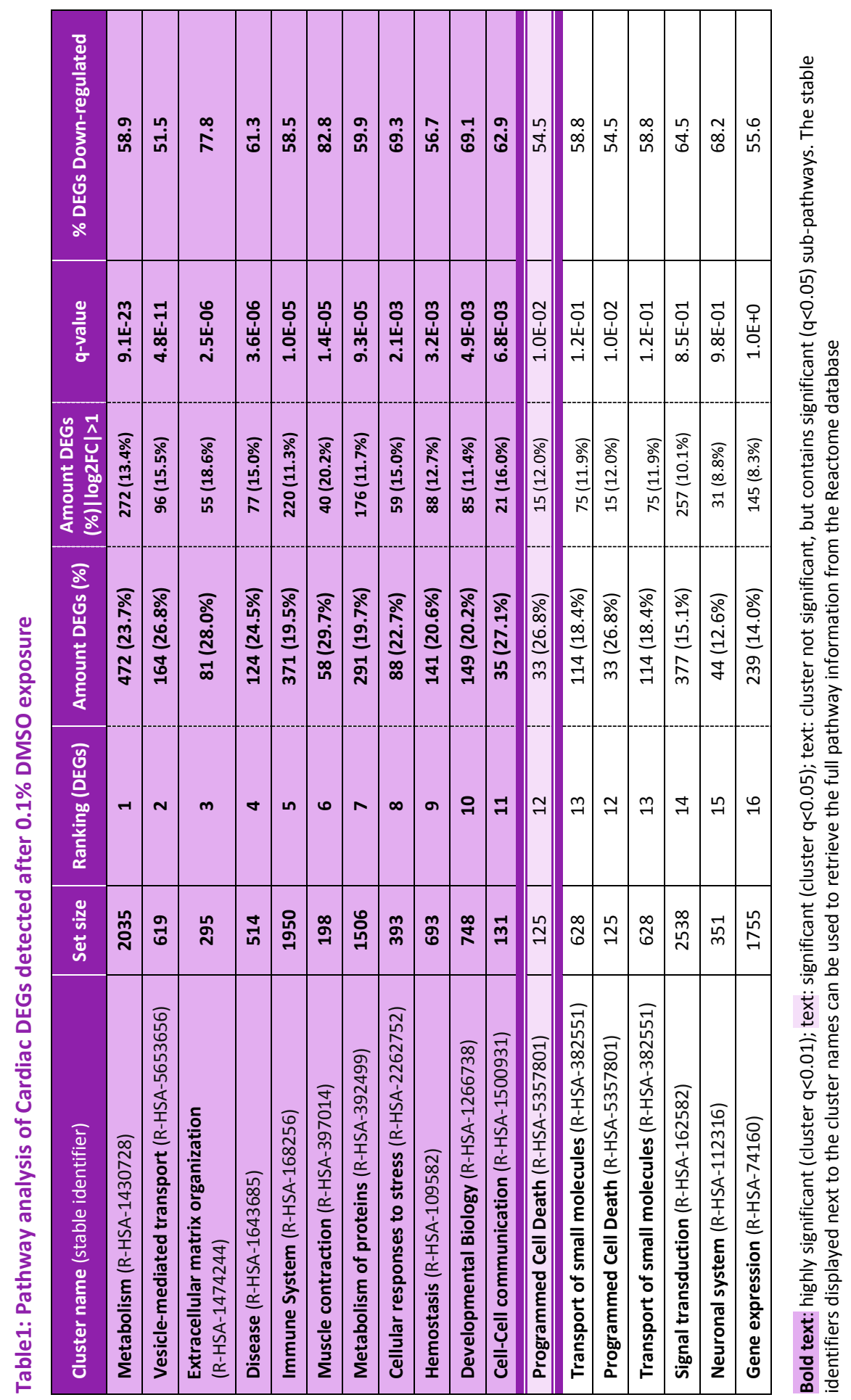


The most significantly affected cluster by DMSO is the "metabolism" cluster in hepatic MTs. Here, most effects were found in pathways "citric acid cycle and respiratory electron transport" (q-value: $3.5^{*} 10^{-10}, 63$ DEGs out of 171 genes, $76.2 \%$ downregulated), "glucose metabolism" (q-value: $9.9 * 10^{-9}, 36$ DEGs out of 77 genes, $80.5 \%$ downregulated) and "metabolism of lipids and lipoproteins" (q-value: $2.9 * 10^{-6}$, 165 DEGs out of 728 genes, 55.2\% downregulated). Changes in lipid and lipoprotein metabolism were not detected in cardiac MTs using DEGs, but similar effects of DMSO were observed for "citric acid cycle and respiratory electron transport" (q-value: $1.3 * 10^{-12}, 58 \mathrm{DEG}$ out of 171 genes, $65.5 \%$ downregulated) and "glucose metabolism" (q-value: $2.8 * 10^{-3}, 20 \mathrm{DEGs}$ out of 77 genes, $55.0 \%$ downregulated), though less genes were downregulated in cardiac MTs compared to hepatic MTs.

Another highly affected cluster by DMSO treatment in both tissue types was "vesicle-mediated transport". DMSO effects in this cluster were mainly detected in processes related to Golgi-mediated protein transport and secretion. Of this process, "ER-to-Golgi anterograde transport" was the most affected (Cardiac: q-value: $2.4 * 10^{-6}$, 38 DEGs out of 134 genes, $68.4 \%$ downregulated; Hepatic: q-value:1.0*10 ${ }^{-5}, 44$ DEGs out of 134 genes, $44.4 \%$ downregulated). This pathway was also part of the cluster "metabolism of proteins", which additionally revealed DMSO effects on "Asparagine Nlinked glycosylation" (Cardiac: q-value: $2.1 * 10^{-6}, 64$ DEGs out of 283 genes, $76.6 \%$ downregulated; Hepatic: q-value: $1.2 * 10^{-7}, 83$ DEGs out of 283 genes, $54.8 \%$ downregulated), which were post-transcriptional protein modifications necessary for transport of proteins from the ER to the Golgi ${ }^{[32]}$.

Though the cluster of "cellular responses to stress" was detected in both tissue types, overrepresented pathways differ. "Cellular senescence" was significantly affected in cardiac MTs (q-value: $2.6 * 10^{-4}$, pathway size: 192, 42 DEGs, 64.3\% downregulated), but not detected during pathway analysis in hepatic MTs. Finally, additional cardiac-specific clusters affected by DMSO were "cell cycle", "DNA repair", "organelle biogenesis and maintenance", but also the highly significant cluster ( $q<$ 0.01) of "chromatin organization". Since the most significantly affected pathways were found in both tissue types, this indicates robust actions of DMSO. 


\section{DMSO effects observed in regulation of gene expression and translation}

Pathways related to regulation of gene expression and translation were already detected during the pathway analysis of cardiac MTs (table 3). Though these pathways were not overrepresented in hepatic MTs (table 4), the amounts of DEGs detected indicated that DMSO was able to influence these processes and could induce biological alterations to the cell model.

Table 3: Cardiac pathways related to transcriptional regulation

\begin{tabular}{|l|c|c|c|c|}
\cline { 2 - 5 } \multicolumn{1}{c|}{} & \multicolumn{3}{c|}{ Cardiac } \\
\cline { 2 - 5 } \multicolumn{1}{c|}{} & $\begin{array}{c}\text { Set } \\
\text { size }\end{array}$ & DEGs & q-value & $\begin{array}{c}\text { \% DEGs down- } \\
\text { regulated }\end{array}$ \\
\hline Gene silencing by RNAs & 134 & $35(26.7 \%)$ & $3.8 \mathrm{E}-05$ & 74.3 \\
Transcriptional regulation by small RNAs & 108 & $32(30.2 \%)$ & $7.6 \mathrm{E}-06$ & 75.0 \\
MicroRNA biogenesis & 13 & $5(41.7 \%)$ & $4.0 \mathrm{E}-02$ & 60.0 \\
Epigenetic regulation of gene expression & 154 & $31(20.5 \%)$ & $6.8 \mathrm{E}-03$ & 61.3 \\
DNA methylation & 68 & $18(27.3 \%)$ & $3.4 \mathrm{E}-03$ & 66.6 \\
\hline
\end{tabular}

Table 4: Hepatic pathways related to transcriptional regulation

\begin{tabular}{|l|c|c|c|c|}
\cline { 2 - 5 } \multicolumn{1}{c|}{} & \multicolumn{3}{c|}{ Hepatic } \\
\cline { 2 - 5 } \multicolumn{1}{c|}{} & $\begin{array}{c}\text { Set } \\
\text { size }\end{array}$ & DEGs & q-value & $\begin{array}{c}\% \text { DEGs down- } \\
\text { regulated }\end{array}$ \\
\hline Gene silencing by RNAs & 134 & $25(19.1 \%)$ & $3.6 \mathrm{E}-01$ & 72.0 \\
Transcriptional regulation by small RNAs & 108 & $24(22.6 \%)$ & $1.4 \mathrm{E}-01$ & 75.0 \\
MicroRNA biogenesis & 13 & $1(8.3 \%)$ & $9.6 \mathrm{E}-01$ & 0.0 \\
Epigenetic regulation of gene expression & 154 & $27(17.9 \%)$ & $4.5 \mathrm{E}-01$ & 77.7 \\
DNA methylation & 68 & $13(19.7 \%)$ & $4.4 \mathrm{E}-01$ & 92.3 \\
\hline
\end{tabular}

\section{DMSO effects observed in regulation by microRNAs}

Tissue-specific influence of DMSO was observed in the sequencing data of miRNAs. Out of 1,105 sequenced cardiac miRNAs, 704 (=63.7\%) were differentially expressed $(D E, F D R<0.05)$ with $59.5 \%$ showing downregulation. Furthermore, the PCA plot (Figure $2 \mathrm{C}$ ) revealed a clear difference between the miRNAs of the treatment groups. In hepatic MTs, out of 1,033 sequenced miRNAs, 186 (=18\%) were DE with approximately half of the miRNAs being upregulated (47.3\%). Furthermore, the PCA plot not only revealed a clear separation between miRNAs of the treatment groups, but indicated also a time-dependent effect. The two principal components (time and dose) represented together more than $95 \%$ of the total variation, leaving only minor effect for other factors. 
To investigate the source of tissue-specific difference, gene expression changes in the process of miRNA biogenesis were investigated in more detail (figure 3). MiRNA biogenesis starts by the transcription of the primary miRNA transcribed by polymerase II (a complex of 11 subunits) or polymerase III (a complex of 10 subunits). In cardiac MTs, polymerase II contained 1 upregulated and 8 downregulated genes. In hepatic MTs, 5 genes were downregulated and 1 was upregulated. Though fewer genes were downregulated in hepatic MTs, the fold changes were larger compared to cardiac MTs. The cleavage of the pri-miRNA into pre-miRNA did not appear affected by DMSO exposure. DICER1 (which cleaves pre-miRNAs) was downregulated in cardiac MTs and upregulated in hepatic MTs, depicting a clear tissue-specific difference in the response to DMSO exposure. Finally, AGO2 (encoding the main component of the miRNA-RISC) was downregulated in cardiac MTs. The changes in this process could induce differences in the cells miRNA content and therefore affect their regulatory function.

In cardiac MTs, the downregulated genes in miRNA biogenesis could explain the extremely high amount of downregulated DE miRNA and indicate extreme deregulation of gene silencing by miRNAs. In hepatic MTs, where the only significant change was the upregulation of DICER1 (Table 3), miRNA biogenesis was not disrupted by DMSO exposure.

To get an indication of the DMSO effects on gene silencing by miRNAs, the miRTarBase database ${ }^{[33]}$, containing experimentally validated microRNA-target interactions (MTI), was used to obtain gene targets of detected DE miRNAs. We only included MTIs with strong evidence (validated by reporter assay, Western blot or qPCR). Of the $704 \mathrm{DE}$ cardiac miRNAs, only 281 (=40\%) could be found in the database, resulting in a total of 2051 gene targets potentially affected by DMSO-induced changes in miRNA gene silencing. For hepatic MTs, targets for 106 DE miRNAs ( $=57 \%)$ were obtained, with a total of 545 potentially affected genes. The obtained gene targets were used to visualize overrepresented pathways using the Reactome Pathway Browser (Supplementary Figure 3). Unexpectedly, overrepresented pathways were located in the same clusters for both tissue types. Most effects were observed for 
"Signal Transduction", "Immune System" and "Gene Expression”. However, extreme deregulation of cardiac MTs and limited information about MTIs is making any downstream analysis on putative affected mRNA irrelevant.

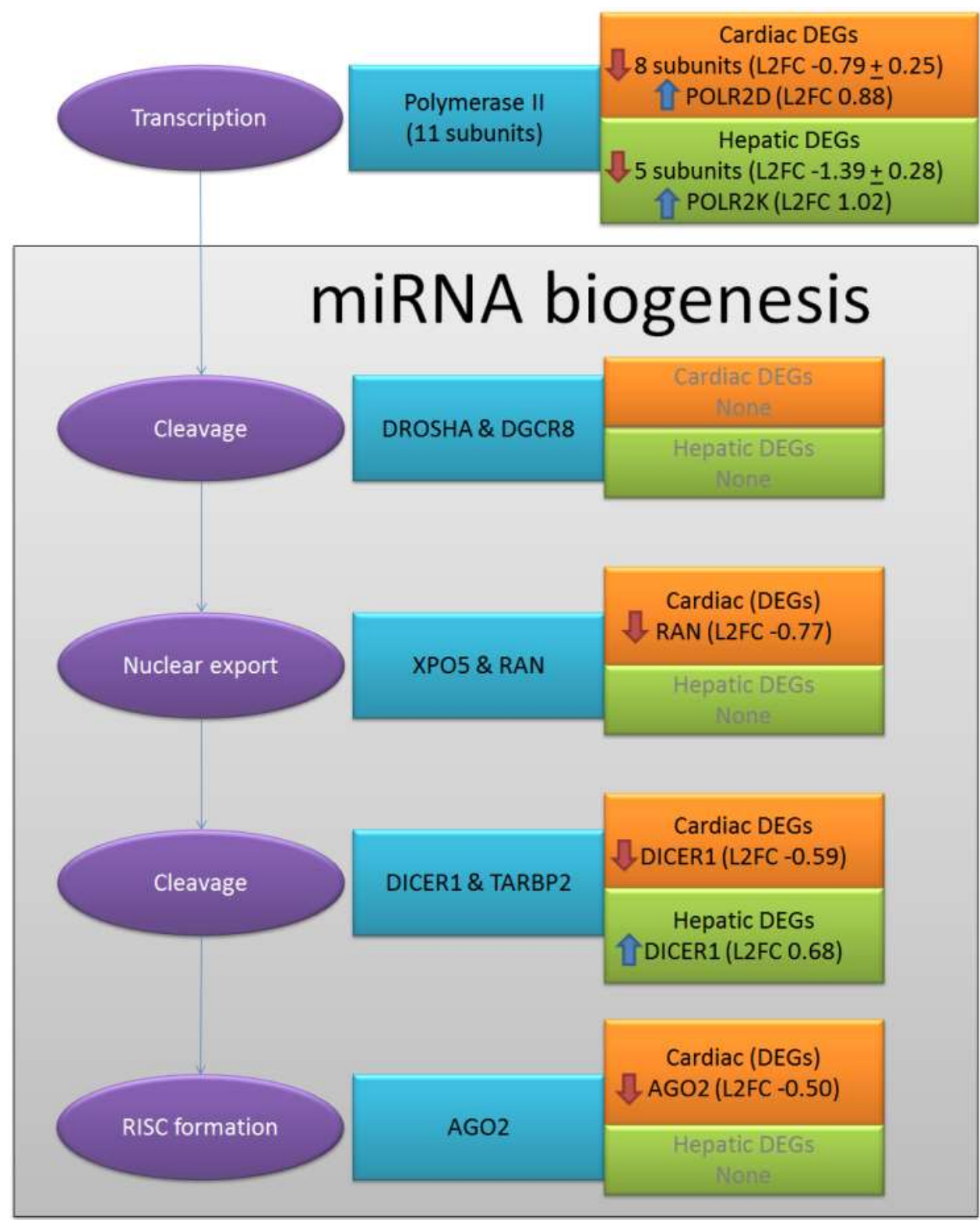

Figure 3: DMSO effect in the process of Gene silencing by RNAs.

DEGs in biogenesis of miRNA. The complete process is divided in sub-processes (purple ovals) and depicting involved genes (blue rectangles) and detected DEGs for cardiac (orange rectangles) and hepatic (green rectangles) samples 


\section{Methylation}

a

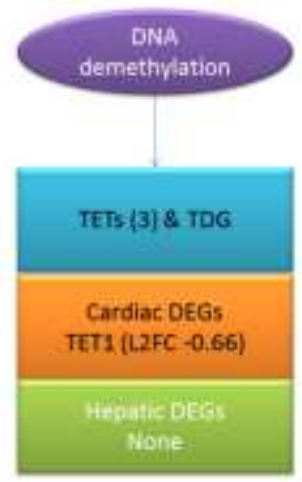

b

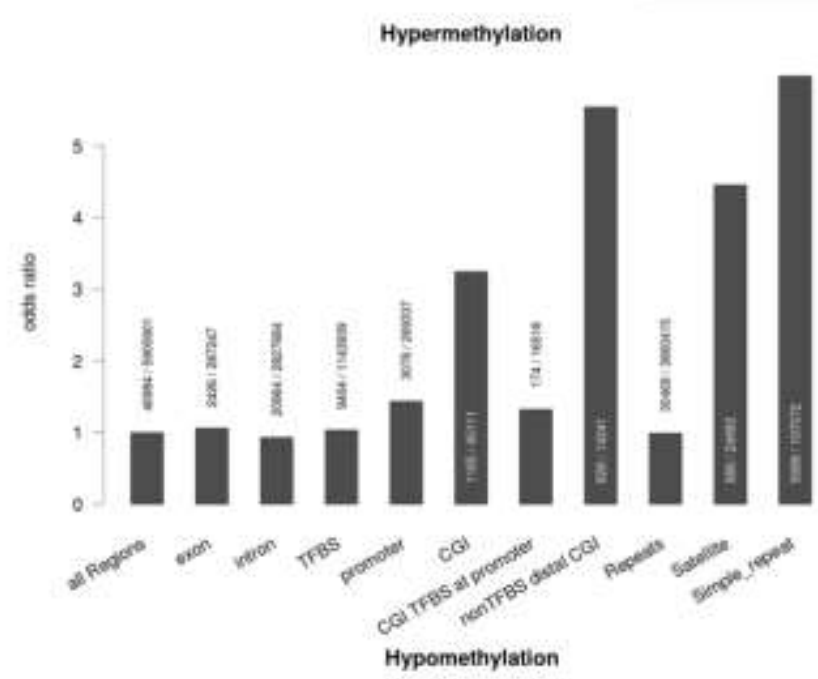

Figure 4: Epigenetic regulation of gene expression. a) DEGs involved in DNA methylation. The process is divided in sub-processes (purple ovals) and depicting involved genes (blue rectangles) and detected DEGs for cardiac (orange rectangles) and hepatic (green rectangles) samples.

b) Relative enrichment of DMRs (DMSO vs UNTR, FDR 5\%) within genomic features. For each feature, the number of overlapping DMRs over the total number of tested windows for the respective feature is depicted. Compared to all genomic regions, hypermethylated regions are enriched for satellites, simple repeats, and CGIs distal to promoters without known regulatory evidence and hypomethylated regions are enriched for simple repeats 


\section{DMSO effects observed in epigenetics}

In order to assess epigenetic alterations introduced by DMSO, we focus on genome wide DNA methylation. Pathway analysis of cardiac gene expression revealed deregulation of DNA methylation pathways. Methyltransferases DNMT1, a key factor for maintenance of DNA methylation, and DNMT3A, facilitating both de novo and maintenance of DNA methylation, were upregulated while TET1, which plays a key role in active de-methylation, was downregulated in cardiac MTs (Figure 4A). Upregulation of epigenetic writers and downregulation of erasers after DMSO treatment pointed towards genomic hypermethylation, which potentially reduced transcriptional activity. In contrast, in hepatic pathway analysis, deregulation of DNA methylation was not observed. Note that, in both tissue types, transcriptional evidence for deregulation of other related epigenetic mechanisms were observed, such as histone methylation, where 16 genes and 13 genes were differentially expressed in cardiac and hepatic MTs respectively.

Whole genome methylation profiling by MeDIP-seq revealed 66,178 differentially methylated regions (DMRs; q-value <0.05) in cardiac MTs. These alterations affected $1.1 \%$ of the covered genome. In line with the observed transcriptional changes of writers and erasers of DNA methylation, $71 \%$ of the DMRs corresponded to gain of methylation (46,984 hypermethylated regions vs 19,194 hypomethylated regions). In contrast, in hepatic MTs, no DMRs passed correction for multiple testing. Furthermore, the PCA plot (Figure 2D) indicated a difference between treatment groups in cardiac MTs but not in hepatic MTs. Together, this illustrated tissue-specific impact of DMSO on the methylome in maturing cardiac MTs, while the mature hepatic MTs appeared unaffected.

In order to analyze the regulatory effects of the DMRs, the regions were annotated with known regulatory features, such as promoters, CpG Islands (CGIs), transcription factor binding sites (TFBS), and different classes of repetitive elements (Figure 4B). Both hypo- and hypermethylated regions enriched specific repeat classes such as satellites (odds ratio of hyper/hypomethylation: 4.4/2.4) and simple repeats 
(hyper/hypomethylation 6.0/9.4 odds ratio), while repetitive elements in general were not enriched. The effect of DMSO on the upregulation of DNMTs and subsequent hypermethylation of repeat sequences was in line with previous findings in mouse embryoid bodies ${ }^{[34]}$. As expected, hypermethylated regions were also highly enriched in CGIs without regulatory evidence, e.g. not overlapping with TFBS and distal to transcription start sites. The odds ratio of these regions over the genome was 5.5. While CGIs in general had an odds ratio of 3.2, there was no enrichment of CGIs overlapping known TFBS at promoters, the regions with the best studied regulatory potential. Just as promoter regions, other features such as exons, introns, and TFBS were affected by hypermethylation at approximately the odds of the captured genomic background (Figure 4B). Although we found specific examples where promoter hypermethylation co-occurs with transcriptional repression, no global correlation between differential promoter methylation and gene expression was observed. Only 148 of the 1,436 genes (10.3\%) featuring hypermethylated promoters were downregulated in DMSO treated cardiac MTs. 86 of the genes (6\%) were overexpressed, contrary to the expected repressive effect of methylation.

Taken together, our data implied a fundamental influence of DMSO on DNA methylation in cardiac MTs, but not in hepatic MTs. The induced alterations affected preferentially satellites and simple repeats, as well as CGIs distal to promoters without known regulatory evidence. The expression of neighboring genes was not significantly correlated to methylation differences. We therefore conclude that transcriptional repression by promoter hypermethylation was not the primary regulatory effect of the altered methylation marks, but the findings indicate a global disruption of DNA methylation mechanisms. 


\section{Discussion}

While high doses of DMSO were initially investigated for medical applications, lower doses are now commonly used as solvent for toxicology or for cryopreservation of cell cultures, oocytes and embryos. In the meantime, biological effects of DMSO have been forgotten or considered negligible. With the evolution of biomedical science towards more sensitive high-throughput techniques and towards new areas of research, including epigenome modifications and microRNA-mediated gene silencing, we evaluated the biological effects of DMSO exposure on the transcriptome and the epigenome.

The first overview of DMSO-induced cross-omics effects, observed through amounts of differentially changed entities and PCA analysis, indicated an increased susceptibility to DMSO effects in cardiac MTs. Differences between the investigated tissue types may relate to the fact that the cardiac model contains iPSC-derived human cardiomyocytes that are still maturing, while the hepatic model contains mature human hepatocytes. Currently, it is not yet possible for iPSC cells to reach an adult-like phenotype ${ }^{[35,36]}$ and the concise review on iPSC-derived human cardiomyocytes of Robertson et al. ${ }^{[37]}$ describes iPSCs to better resemble fetal cardiomyocytes (regarding structure, proliferation, metabolism and electrophysiology) rather than adult cardiomyocytes. Our observations of DMSO effects, notably the extreme epigenetics modifications in fetal-like cardiac cells, are particularly relevant for cryopreservation during assisted reproductive technology (embryos, oocytes and sperm cells). Another difference to be considered between our two cell models is the number of donors. Indeed, while the cardiac MTs are composed of IPSC-derived cardiomyocytes from a single donor (cocultured with fibroblasts also from 1 donor), hepatic MTs contain mature hepatocytes from 10 donors (co-cultured with Kupffer cells of 1 donor). While a single-donor model is more comparable to an in vivo situation then a multi-donor model, single-donor cardiac MTs might be more susceptible for bias due to genotype sensitivity than our multi-donor hepatic MTs that have the ability to even out these effects. 
DMSO effects on cellular processes were analyzed using full transcriptome data . 2,051 DEGs were identified for cardiac MTs and 2711 DEGs for hepatic MTs. In both tissue types, downregulations are found for $60 \%$ of DEGs. Pathway overrepresentation analysis using DEGs, highlights similar DMSO effects in both tissue types. This indicates robust actions of DMSO that may possibly be extrapolated to other tissues. Observed changes in cellular processes include changes in mitochondrial pathways linked to ROS production and cellular ATP generation. DMSO is a known radical scavenger ${ }^{[38]}$. While this property of DMSO may provide protection against high levels of ROS, at normal or decreased levels of ROS, it may hamper basal cell metabolism by scavenging electrons needed for ATP production ${ }^{[39]}$. The resulting decrease in ATP content was observed in our two DMSO treated cell models (Supplementary Figure 2). Cardiac MTs showed a steep decrease over time ( $87 \%$ decrease after 2 weeks of exposure), while the ATP content of hepatic MTs show a small decrease initially (36\% decrease in the first $72 \mathrm{~h}$ ), after which ATP content slowly recovered to baseline level. Different downstream effects are relevant depending on the biological application of DMSO. For instance, for cell assays and toxicological research, changes in the amount of ROS or free energy in the cell impact on the capability of the cell to deal with induced stresses, thereby potentially leading to erroneous interpretation of results from in vitro assays. Within assisted reproductive technology, the ATP content is a good predictor of embryo viability. The DMSO-induced ATP decrease, especially in the cleavage-stage, can induce downstream effects that may disrupt cellular function, implantation ability and fetal development ${ }^{[40,41]}$. DMSO-induced reduction in implantation rates and pregnancy losses were already observed from animal models ${ }^{[42]}$.

Next to overlap in DMSO-affected processes between the tissue types, pathway analysis also revealed tissue-specific differences. The higher amount of affected processes detected in cardiac MTs may indicate increased susceptibility of cardiac MTs to the effects of DMSO, though there might also be a statistical bias due to different amount of DEGs used in the pathway analysis. The most significant difference is the detection of "chromatin organization" in cardiac MTs, while this cluster is not observed in hepatic MTs. Cardiac MTs also show more effects in other processes that regulate 
gene expression. Results of miRNA analysis revealed complete deregulation of cardiac miRNA biogenesis and miRNA content. Though changes in hepatic miRNA biogenesis were minimal, still $18 \%$ of detected miRNAs were significantly changed by DMSO exposure. Genome-wide methylation effects upon DMSO treatment show large tissuespecific variation. While there is no significant difference with respect to hepatic MTs, strong effects are observed with cardiac MTs, in particular with respect to specific repetitive sequences such as satellites and simple repeats. DNA repetitive elements account for $50 \%$ of the human genome and methylation in these regions suppresses their mobility and maintains genomic stability. Changes in the methylation status of repeats after DMSO treatment might destabilize the genome, implying phenotypic and pathological consequences. Indeed, recently, methylation changes of repetitive sequences, in particular satellite repeat elements, have been found in cardiomyopathic heart tissue compared to normal heart tissue ${ }^{[43]}$. Furthermore, it has been shown that DMSO changes genome-wide DNA methylation profiles dependent on specific gene loci. This may be due to drastic stimulation of DNMT1 and DNMT3. We observed upregulation of DNMTs, but literature also reported increased protein levels and catalytic activity by interaction of DMSO with their enzymes substrates (DNA and AdoMet) ${ }^{[34,44,45]}$. Furthermore, it is also possible that DMSO acts as a methyl donor to induce hypermethylation ${ }^{[46]}$. However, it has to be kept in mind that there is an interplay between DNA methylation and chromatin configuration, which is regulated by more processes than just DNMTs. Overall, we speculate the effects of DMSO on methylation changes to be a global deregulation characterized by a genome wide hypermethylation. Cell mechanisms in charge of removing detrimental methylations at important regions, such as TFBS, may then have removed the methylation changes with a negative outcome on cell survival, leaving an over-representation of DMR in the repetitive elements. This is in analogy to mutation rates, which are higher for repetitive elements since they are less subjected to natural selection and DNA repair mechanisms ${ }^{[47]}$. We believe that the methylation difference between mature hepatic and maturing cardiac cells may be detrimental for cryopreserved cells and especially for oocytes and embryos. Previously reported DMSO effects on oocytes and embryos are limited to changes in gene expression in animal models ${ }^{[42,48-51]}$. 
We now showed, for the first time, the influence of DMSO on the epigenome of human cells in vitro. It is uncertain if exposed cells can recover from temporary DMSO exposures. Increased survival has been documented for embryos after withdrawal from $2 \%$ DMSO for less than $24 \mathrm{~h}$, though this was not the case with exposures longer then $48 \mathrm{~h}^{[42]}$. While methylation changes have an adaptive nature, they may also be persistent. From research conducted on the Dutch famine cohort, it is known that the periconceptional period (before conception until early pregnancy) is crucial for establishing and maintaining epigenetic marks. During this period epigenetic marks present on the parental DNA are removed to produce a totipotent zygote which is reprogrammed after implantation ${ }^{[52]}$. Exposures during this time may induce persistent epigenetic differences, which can result in disease later in life or possibly be inherited by the offspring to induce transgenerational effects ${ }^{[53-57]}$. It is already well known that infants conceived through assisted reproductive technologies are prone to be born preterm, have low birth weight and even have a significantly increased risk of birth defects ${ }^{[58,59]}$. Furthermore, some short-term health outcomes were slightly poorer in children conceived by IVF, though overall the outcomes were positive ${ }^{[57,60]}$. Regarding long-term health and development, though available data is limited, cardiovascular and metabolic risk factors that may ultimately result in chronic cardiometabolic disease have been indicated ${ }^{[57]}$. Furthermore, also epigenetic risks have been debated, mostly focusing on methylation and imprinting. For the most studied imprinting disorders Beck-with-Wiedemann syndrome and Angelman syndrome, the incidence was higher for children born after assisted reproductive technologies compared to natural conception ${ }^{[56,61-65]}$. While there are many factors contributing to these abnormalities, (decreased infertility, higher age of the parents, technical interference with a biological process, etc.) DMSO-induced effects may be one of them.

In the last decade, improvements made in artificial reproductive technology greatly increased the success rate of IVF treatments. Especially, improved cryopreservation techniques (transition to vitrification) aided greatly. Though not proven yet, increased success rates are reported when implanting previously frozen embryos ${ }^{[66-68]}$. This induced the "freeze all" trend in the clinic, which greatly increases the need for 
understanding consequences of cryopreservation at the molecular level. Furthermore, while storing frozen oocytes was initially intended for women threatened by decreased fertility due to disease, in our carrier-focused society, it has also become a method to

prevent age-related infertility for women who want to delay motherhood ${ }^{[69]}$. The most recent development in assisted reproductive technology is the emerging of concerns about DMSO effects. While there is still controversy on this topic, the field is moving towards DMSO-free methods. However, DMSO-free does not automatically mean safe because of the use of other cryoprotectants ${ }^{[70-72]}$.

\section{Conclusion}

Our study highlights the capability of DMSO to induce changes in cellular processes in both cardiac and hepatic cells, but more severely, induce alterations in miRNA and epigenetic landscape in the 3D maturing cardiac model. The changes in cellular processes can have consequences for conclusions drawn from cell assays and therefore also in any application of these findings (e.g. false negative drug toxicity conclusions). Furthermore, the extreme changes in miRNA and alterations in the epigenetic landscape may pose a threat, especially for assisted reproductive technology. Genome-wide hypermethylation induced by global deregulation of methylation mechanisms, especially when it affects genes important in development, may have negative consequences directly, later in life or possibly in later generations.

Overall, use of DMSO should be avoided where possible. However, for the time being, DMSO is indispensable within biotechnological applications. In these cases, the effects that DMSO may have should be considered and the concentration should be kept as low as possible, because even at low concentrations DMSO is not inert. 


\section{Supplementary materials}

Supplementary figure 1: PCA plot to detect outliers.

$\mathrm{ftp}: / /$ web.tgx.unimaas.nl/mverheijen/Chapter2/Supplementary_figure1.docx

Supplementary figure 2: Changes in microtissue ATP content over time.

ftp://web.tgx.unimaas.nl/mverheijen/Chapter2/Supplementary_figure2.docx

Supplementary figure 3: Indication of biological effects due to miRNA changes.

ftp://web.tgx.unimaas.nl/mverheijen/Chapter2/Supplementary_figure3.docx

Supplementary table 1: Hierarchically ordered overrepresented pathways (using DEGs) in cardiac MTs after 0.1\% DMSO exposure

ftp://web.tgx.unimaas.nl/mverheijen/Chapter2/Supplementary_table1.xlsx

Supplementary table 2: Hierarchically ordered overrepresented pathways (using DEGs) in hepatic MTs after 0.1\% DMSO exposure

ftp://web.tgx.unimaas.nl/mverheijen/Chapter2/Supplementary_table2.xlsx

Supplementary table 3: Hierarchically ordered overrepresented pathways (using DEPs) in cardiac MTs after 0.1\% DMSO exposure ftp://web.tgx.unimaas.nl/mverheijen/Chapter2/Supplementary_table3.xlsx

Supplementary table 4: Hierarchically ordered overrepresented pathways (using DEPs) in hepatic MTs after 0.1\% DMSO exposure ftp://web.tgx.unimaas.nl/mverheijen/Chapter2/Supplementary_table4.xlsx

Supplementary data: Proteomics Methods \& Results ftp://web.tgx.unimaas.nl/mverheijen/Chapter2/Supplementary_data.xlsx 


\section{References}

1 Galvao, J. et al. Unexpected low-dose toxicity of the universal solvent DMSO. Faseb J 28, 1317-1330, doi:10.1096/fj.13-235440 (2014).

2 Sumida, K. et al. Effects of DMSO on gene expression in human and rat hepatocytes. Hum Exp Toxicol 30, 1701-1709, doi:10.1177/0960327111399325 (2011).

3 Yuan, Y. et al. Efficient long-term cryopreservation of pluripotent stem cells at-80 degrees C. Sci Rep-Uk 6, doi:ARTN 34476 10.1038/srep34476 (2016).

4 FDA. <http://www.accessdata.fda.gov/cms_ia/importalert_169.html>

5 Brobyn, R. D. The human toxicology of dimethyl sulfoxide. Ann N Y Acad Sci 243, 497-506 (1975).

6 Rubin, L. F. Toxicologic update of dimethyl sulfoxide. Ann N Y Acad Sci 411, 6-10 (1983).

7 Smith, E. R., Hadidian, Z. \& Mason, M. M. The single--and repeated--dose toxicity of dimethyl sulfoxide. Ann N Y Acad Sci 141, 96-109 (1967).

8 FDA. <www.fda.gov/downloads/drugs/guidancecomplianceregulatoryinformation/guidances/ ucm073395.pdf> (2012).

9 Jacob, S. W. \& Herschler, R. Pharmacology of DMSO. Cryobiology 23, 14-27 (1986).

10 Edgar, D. H. \& Gook, D. A. A critical appraisal of cryopreservation (slow cooling versus vitrification) of human oocytes and embryos. Hum Reprod Update 18, 536-554, doi:10.1093/humupd/dms016 (2012).

11 Denko, C. W., Goodman, R. M., Miller, R. \& Donovan, T. Distribution of dimethyl sulfoxide-35S in the rat. Ann N Y Acad Sci 141, 77-84 (1967).

12 Yu, Z. W. \& Quinn, P. J. Dimethyl sulphoxide: a review of its applications in cell biology. Biosci Rep 14, 259-281 (1994).

13 Deng, Z. H. et al. Topical diclofenac therapy for osteoarthritis: a meta-analysis of randomized controlled trials. Clin Rheumatol 35, 1253-1261, doi:10.1007/s10067-015-3021-z (2016).

14 Fuller, P. \& Roth, S. Diclofenac sodium topical solution with dimethyl sulfoxide, a viable alternative to oral nonsteroidal anti-inflammatories in osteoarthritis: review of current evidence. J Multidiscip Healthc 4, 223-231, doi:10.2147/JMDH.S23209 (2011).

15 Kuepfer, L. et al. A model-based assay design to reproduce in vivo patterns of acute drug-induced toxicity. Arch Toxicol, 1-3 (2017).

16 Bolger, A. M., Lohse, M. \& Usadel, B. Trimmomatic: a flexible trimmer for Illumina sequence data. Bioinformatics 30, 2114-2120, doi:10.1093/bioinformatics/btu170 (2014).

17 Andrews, S. FastQC: a quality control tool for high throughput sequence data. http://www.bioinformatics.babraham.ac.uk/projects/fastqc (2010).

18 Li, B. \& Dewey, C. N. RSEM: accurate transcript quantification from RNA-Seq data with or without a reference genome. Bmc Bioinformatics 12, doi:Artn 323 10.1186/1471-2105-12-323 (2011).

19 Langmead, B. \& Salzberg, S. L. Fast gapped-read alignment with Bowtie 2. Nat Methods 9, 357-U354, doi:10.1038/Nmeth.1923 (2012).

20 Love, M. I., Huber, W. \& Anders, S. Moderated estimation of fold change and dispersion for RNA-seq data with DESeq2. Genome Biol 15, doi:ARTN 550 10.1186/s13059-014-0550-8 (2014).

21 Stocks, M. B. et al. The UEA sRNA workbench: a suite of tools for analysing and visualizing next generation sequencing microRNA and small RNA datasets. Bioinformatics 28, 2059-2061, doi:10.1093/bioinformatics/bts311 (2012).

22 Caiment, F., Gaj, S., Claessen, S. \& Kleinjans, J. High-throughput data integration of RNA-miRNA-circRNA reveals novel insights into mechanisms of benzo[a]pyrene-induced carcinogenicity. Nucleic Acids Res 43, 2525-2534, doi:10.1093/nar/gkv115 (2015).

23 Prufer, K. et al. PatMaN: rapid alignment of short sequences to large databases. Bioinformatics 24, 15301531, doi:10.1093/bioinformatics/btn223 (2008).

24 Taiwo, O. et al. Methylome analysis using MeDIP-seq with low DNA concentrations. Nature protocols 7, 617 (2012).

$25 \mathrm{Li}, \mathrm{H}$. \& Durbin, R. Fast and accurate long-read alignment with Burrows-Wheeler transform. Bioinformatics 26, 589-595, doi:10.1093/bioinformatics/btp698 (2010).

26 Lienhard, M. et al. QSEA-modelling of genome-wide DNA methylation from sequencing enrichment experiments. Nucleic Acids Res 45, e44, doi:10.1093/nar/gkw1193 (2017).

27 Consortium, E. P. An integrated encyclopedia of DNA elements in the human genome. Nature 489, 57-74, doi:10.1038/nature11247 (2012).

28 Aryee, M. J. et al. Minfi: a flexible and comprehensive Bioconductor package for the analysis of Infinium DNA methylation microarrays. Bioinformatics 30, 1363-1369, doi:10.1093/bioinformatics/btu049 (2014). 
29 Hinrichs, A. S. et al. The UCSC Genome Browser Database: update 2006. Nucleic Acids Res 34, D590-598, doi:10.1093/nar/gkj144 (2006).

30 Kamburov, A. et al. ConsensusPathDB: toward a more complete picture of cell biology. Nucleic Acids Res 39, D712-D717, doi:10.1093/nar/gkq1156 (2011).

31 Fabregat, A. et al. The Reactome pathway Knowledgebase. Nucleic Acids Res 44, D481-D487, doi:10.1093/nar/gkv1351 (2016).

32 Lederkremer, G. Z. Glycoprotein folding, quality control and ER-associated degradation. Curr Opin Struc Biol 19, 515-523, doi:10.1016/j.sbi.2009.06.004 (2009).

33 Chou, C. H. et al. miRTarBase 2016: updates to the experimentally validated miRNA-target interactions database. Nucleic Acids Res 44, D239-247, doi:10.1093/nar/gkv1258 (2016).

34 Iwatani, M. et al. Dimethyl sulfoxide has an impact on epigenetic profile in mouse embryoid body. Stem Cells 24, 2549-2556, doi:10.1634/stemcells.2005-0427 (2006).

35 Nunes, S. S., Miklas, J. W. \& Radisic, M. Maturation of stem cell-derived human heart tissue by mimicking fetal heart rate. Future Cardiol 9, 751-754, doi:10.2217/fca.13.71 (2013).

36 Batalov, I. \& Feinberg, A. W. Differentiation of Cardiomyocytes from Human Pluripotent Stem Cells Using Monolayer Culture. Biomark Insights 10, 71-76 (2015).

37 Robertson, C., Tran, D. D. \& George, S. C. Concise Review: Maturation Phases of Human Pluripotent Stem Cell-Derived Cardiomyocytes. Stem Cells 31, 829-837, doi:10.1002/stem.1331 (2013).

38 Eberhardt, M. K. \& Colina, R. The reaction of $\mathrm{OH}$ radicals with dimethyl sulfoxide. A comparative study of Fenton's reagent and the radiolysis of aqueous dimethyl sulfoxide solutions. The Journal of Organic Chemistry 53, 1071-1074 (1988).

39 Zorov, D. B., Juhaszova, M. \& Sollott, S. J. Mitochondrial reactive oxygen species (ROS) and ROS-induced ROS release. Physiol Rev 94, 909-950, doi:10.1152/physrev.00026.2013 (2014).

40 Lane, M. \& Gardner, D. K. Understanding cellular disruptions during early embryo development that perturb viability and fetal development. Reprod Fert Develop 17, 371-378, doi:10.1071/Rd04102 (2005).

41 Benkhalifa, M. et al. Mitochondria: Participation to infertility as source of energy and cause of senescence. Int J Biochem Cell B 55, 60-64, doi:10.1016/j.biocel.2014.08.011 (2014).

42 Kang, M. H. et al. The cytotoxic effects of dimethyl sulfoxide in mouse preimplantation embryos: a mechanistic study. Theranostics 7, 4735-4752, doi:10.7150/thno.21662 (2017).

43 Haider, S. et al. The landscape of DNA repeat elements in human heart failure. Genome Biol 13, doi:ARTN R90 10.1186/gb-2012-13-10-R90 (2012).

44 Yokochi, T. \& Robertson, K. D. Dimethyl sulfoxide stimulates the catalytic activity of de novo DNA methyltransferase 3a (Dnmt3a) in vitro. Bioorg Chem 32, 234-243, doi:10.1016/j.bioorg.2004.04.005 (2004).

45 Thaler, R., Spitzer, S., Karlic, H., Klaushofer, K. \& Varga, F. DMSO is a strong inducer of DNA hydroxymethylation in pre-osteoblastic MC3T3-E1 cells. Epigenetics-Us 7, 635-651, doi:10.4161/epi.20163 (2012).

46 Kawai, K., Li, Y. S., Song, M. F. \& Kasai, H. DNA methylation by dimethyl sulfoxide and methionine sulfoxide triggered by hydroxyl radical and implications for epigenetic modifications. Bioorg Med Chem Lett 20, 260-265, doi:10.1016/j.bmcl.2009.10.124 (2010).

47 Nishant, K. T., Singh, N. D. \& Alani, E. Genomic mutation rates: what high-throughput methods can tell us. Bioessays 31, 912-920, doi:10.1002/bies.200900017 (2009).

48 Stinshoff, H., Wilkening, S., Hanstedt, A., Bollwein, H. \& Wrenzycki, C. Dimethylsulfoxide and conjugated linoleic acids affect bovine embryo development in vitro. Reprod Fert Develop 26, 502-510, doi:10.1071/Rd12372 (2014).

49 Azari, M., Kafi, M., Ebrahimi, B., Fatehi, R. \& Jamalzadeh, M. Oocyte maturation, embryo development and gene expression following two different methods of bovine cumulus-oocyte complexes vitrification. Vet Res Commun 41, 49-56, doi:10.1007/s11259-016-9671-8 (2017).

50 Li, X., Wang, Y. K., Song, Z. Q., Du, Z. Q. \& Yang, C. X. Dimethyl Sulfoxide Perturbs Cell Cycle Progression and Spindle Organization in Porcine Meiotic Oocytes. Plos One 11, doi:ARTN e0158074 10.1371/journal.pone.0158074 (2016).

51 Zhou, D. J. et al. Effects of dimethyl sulfoxide on asymmetric division and cytokinesis in mouse oocytes. Bmc Dev Biol 14, doi:Artn 28 10.1186/1471-213x-14-28 (2014).

$52 \mathrm{Xu}, \mathrm{Q} . \mathrm{H}$. \& Xie, W. Epigenome in Early Mammalian Development: Inheritance, Reprogramming and Establishment. Trends Cell Biol 28, 237-253, doi:10.1016/j.tcb.2017.10.008 (2018).

53 Heijmans, B. T. et al. Persistent epigenetic differences associated with prenatal exposure to famine in humans. P Natl Acad Sci USA 105, 17046-17049, doi:10.1073/pnas.0806560105 (2008). 
54 Tobi, E. W. et al. DNA methylation differences after exposure to prenatal famine are common and timing- and sex-specific. Hum Mol Genet 18, 4046-4053, doi:10.1093/hmg/ddp353 (2009).

55 Veenendaal, M. V. E. et al. Transgenerational effects of prenatal exposure to the 1944-45 Dutch famine. Bjog-Int J Obstet Gy 120, 548-554, doi:10.1111/1471-0528.12136 (2013).

56 De Rycke, M., Liebaers, I. \& Van Steirteghem, A. Epigenetic risks related to assisted reproductive technologies - Risk analysis and epigenetic inheritance. Hum Reprod 17, 2487-2494, doi:DOI 10.1093/humrep/17.10.2487 (2002).

57 Hart, R. \& Norman, R. J. The longer-term health outcomes for children born as a result of IVF treatment: Part IGeneral health outcomes. Hum Reprod Update 19, 232-243, doi:10.1093/humupd/dms062 (2013).

58 Hansen, M., Bower, C., Milne, E., de Klerk, N. \& Kurinczuk, J. J. Assisted reproductive technologies and the risk of birth defects - a systematic review. Hum Reprod 20, 328-338, doi:10.1093/humrep/deh593 (2005).

59 Bower, C. \& Hansen, M. Assisted reproductive technologies and birth outcomes: overview of recent systematic reviews. Reprod Fert Develop 17, 329-333, doi:10.1071/Rd04095 (2005).

60 Pelkonen, S. et al. Physical health of singleton children born after frozen embryo transfer using slow freezing: a 3-year follow-up study. Hum Reprod 30, 2411-2418, doi:10.1093/humrep/dev203 (2015).

61 Maher, E. R., Afnan, M. \& Barratt, C. L. Epigenetic risks related to assisted reproductive technologies: Epigenetics, imprinting, ART and icebergs? Hum Reprod 18, 2508-2511, doi:10.1093/humrep/deg486 (2003).

62 Laprise, S. L. Implications of Epigenetics and Genomic Imprinting in Assisted Reproductive Technologies. Mol Reprod Dev 76, 1006-1018, doi:10.1002/mrd.21058 (2009).

63 Manipalviratn, S., DeCherney, A. \& Segars, J. Imprinting disorders and assisted reproductive technology. Fertil Steril 91, 305-315, doi:10.1016/j.fertnstert.2009.01.002 (2009).

64 Thompson, J. G., Kind, K. L., Roberts, C. T., Robertson, S. A. \& Robinson, J. S. Epigenetic risks related to assisted reproductive technologies - Short- and long-term consequences for the health of children conceived through assisted reproduction technology: more reason for caution? Hum Reprod 17, 27832786, doi:DOI 10.1093/humrep/17.11.2783 (2002).

65 Uyar, A. \& Seli, E. The impact of assisted reproductive technologies on genomic imprinting and imprinting disorders. Curr Opin Obstet Gyn 26, 210-221, doi:10.1097/Gco.0000000000000071 (2014).

66 Barnhart, K. T. Introduction: Are we ready to eliminate the transfer of fresh embryos in in vitro fertilization? Fertil Steril 102, 1-2, doi:10.1016/j.fertnstert.2014.05.024 (2014).

67 Chen, Z. J. et al. Fresh versus Frozen Embryos for Infertility in the Polycystic Ovary Syndrome. New Engl J Med 375, 523-533, doi:10.1056/NEJMoa1513873 (2016).

68 Vuong, L. N. et al. IVF Transfer of Fresh or Frozen Embryos in Women without Polycystic Ovaries. New Engl J Med 378, 137-147, doi:10.1056/NEJMoa1703768 (2018).

69 Petropanagos, A. in Oncofertility 223-235 (Springer, 2010).

70 Gardner, D. K. \& Kelley, R. L. Impact of the IVF laboratory environment on human preimplantation embryo phenotype. J Dev Orig HIth Dis 8, 418-435, doi:10.1017/S2040174417000368 (2017).

71 Westphal, J. R., Gerritse, R., Braat, D. D. M., Beerendonk, C. C. M. \& Peek, R. Complete protection against cryodamage of cryopreserved whole bovine and human ovaries using DMSO as a cryoprotectant. J Assist Reprod Gen 34, 1217-1229, doi:10.1007/s10815-017-0963-x (2017).

72 De Munck, N., Vajta, G. \& Rienzi, L. in Preventing Age Related Fertility Loss 87-101 (Springer, 2018). 


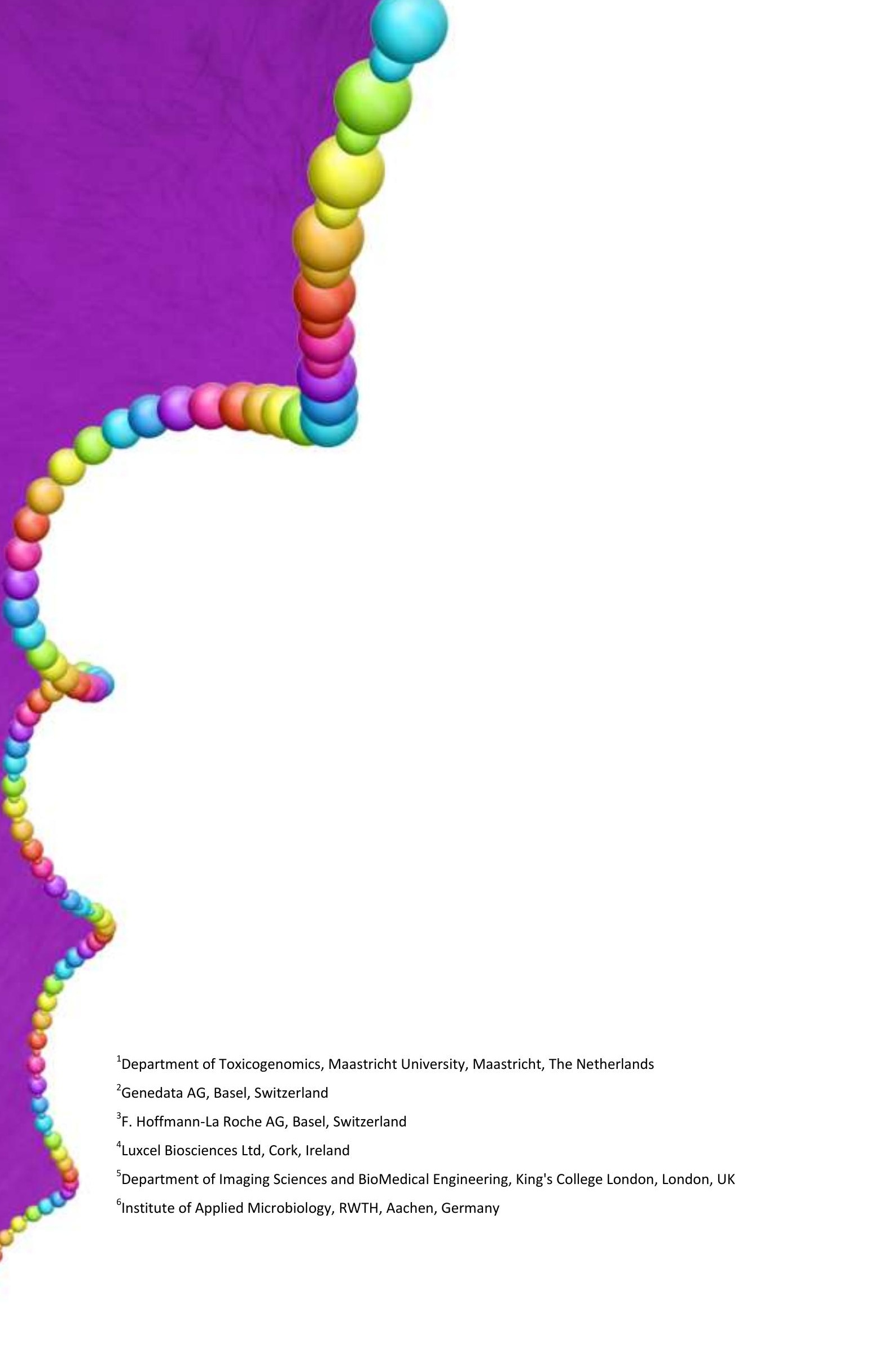




\section{Chapter 3}

\section{Bringining in vitroanalysis closer to în vivo:}

Studying doxorubicin toxicity and associated mechanisms in 3D human microtissues with DBDK-based dose modelling

Marcha C.T. Verheijen ${ }^{1}$

Y. Schrooders ${ }^{1}$

H. Gmuender ${ }^{2}$

R. Nudischer ${ }^{3}$

O. Clayton ${ }^{3}$

J. Hynes ${ }^{4}$

S. Niederer ${ }^{5}$

H. Cordes ${ }^{6}$

L. Kuepfer 6

J. Kleinjans $^{1}$

F. Caiment ${ }^{1}$

Published:

Toxicology Letters, 2018

Volume 294, Pages 184-192 


\section{Abstract}

Doxorubicin (DOX) is a chemotherapeutic agent of which the medical use is limited due to cardiotoxicity. While acute cardiotoxicity is reversible, chronic cardiotoxicity is persistent or progressive, dose-dependent and irreversible. While DOX mechanisms of action are not fully understood yet, 3 toxicity processes are known to occur in vivo: cardiomyocyte dysfunction, mitochondrial dysfunction and cell death. We present an in vitro experimental design aimed at detecting DOX-induced cardiotoxicity by obtaining a global view of the induced molecular mechanisms through RNAsequencing. To better reflect the in vivo situation, human 3D cardiac microtissues were exposed to physiologically-based pharmacokinetic (PBPK) relevant doses of DOX for 2 weeks. We analysed a therapeutic and a toxic dosing profile. Transcriptomics analysis revealed significant gene expression changes in pathways related to "striated muscle contraction" and "respiratory electron transport", thus suggesting mitochondrial dysfunction as an underlying mechanism for cardiotoxicity. Furthermore, expression changes in mitochondrial processes differed significantly between the doses. Therapeutic dose reflects processes resembling the phenotype of delayed chronic cardiotoxicity, while toxic doses resembled acute cardiotoxicity. Overall, these results demonstrate the capability of our innovative in vitro approach to detect the three known mechanisms of DOX leading to toxicity, thus suggesting its potential relevance for reflecting the patient situation. Our study also demonstrated the importance of applying physiologically relevant doses during toxicological research, since mechanisms of acute and chronic toxicity differ. 


\section{Introduction}

Doxorubicin (DOX, also known by the brand name Adriamycin) is a chemotherapeutic agent belonging to the class of anthracyclines, which are cytostatic antibiotics. DOX was isolated from Streptomyces peucetius var. caesius in $1967^{[1]}$ and it is still one of the most frequently used anti-cancer agents for treating a variety of cancers (e.g. hematological cancers, soft-tissue tumors, and solid tumors), even though it causes severe side effects. Next to nausea, vomiting, alopecia, myelosuppression, stomatitis, and gastrointestinal disturbances, DOX is known to induce cardiotoxicity. Cardiotoxicity is classified as acute or chronic. Acute cardiotoxicity may already occur during treatment with a single high dose or within 2-3 days after multiple DOX treatments ${ }^{[2,3]}$. Though its prevalence is higher than for chronic cardiotoxicity, these side effects are reversible and clinically manageable. A chronic cardiotoxic phenotype may emerge between one month and decades after DOX treatment. This persistent or progressive cardiotoxicity is dose-dependent and irreversible. In cases where congestive heart failure has developed, the prognosis for the patient is poor, with a mortality rate of $50 \%$ within one year ${ }^{[3-7]}$. Given this dose-dependency, a maximum cumulative dose of $450-600 \mathrm{mg} / \mathrm{m}^{2}$ is recommended for $\mathrm{DOX}^{[8,9]}$.

DOX mechanisms of action are not fully understood yet. Nevertheless, it is generally accepted that the main mode of action is related to killing dividing cells. These effects are more severe for tumor cells since these cells divide more rapidly than non-cancer cells. However, DOX is not tumor cell-specific and can accumulate in the nucleus and mitochondria of heart, liver and blood cells, thereby contributing to toxic side effects, in particular chronic cardiotoxicity, even at therapeutic doses ${ }^{[5]}$. DOX has been found to 1) intercalate into DNA, 2) target DNA topoisomerases, and 3) generate reactive oxygen species (ROS) ${ }^{[10-12]}$. The first two processes inhibit unwinding of DNA, DNA replication, RNA transcription and protein biosynthesis. As a result, proliferation of dividing cells is also inhibited ${ }^{[9,13]}$. This is thought to be the efficacy of the anti-cancer effects of DOX, while ROS generation is mainly ascribed to toxic effects ${ }^{[14]}$. ROS can be generated as a result of mitochondrial dysfunction, but also by the oxidative semiquinone formed at complex I of the electron transport chain (ETC) during DOX 
metabolism. The induced oxidative stress may damage cells and cause cell death ${ }^{[8,11]}$. Other detrimental actions of DOX can be related to death of non-cancer cells or to decreased cardiomyocyte functioning, which may partly result from mitochondrial dysfunction causing an imbalance in cellular energetics. Therefore, any effect of DOX on mitochondria structure or function can cause cardiomyocyte dysfunction and thereby cardiotoxicity ${ }^{[15]}$. The detrimental actions of DOX, known to occur in vivo, can thus be summarized into 3 toxicity processes: cardiomyocyte dysfunction, mitochondrial dysfunction and cell death. However, it should be noted that the split between efficacy and detrimental actions is not fully justified because of overlapping processes, such as oxidative stress and cell death.

To predict molecular mechanisms underlying long term toxicity, toxicological risk assessment traditionally relies on animal models ${ }^{[16]}$. However, notably due to increasing ethical pressure, the field has to reduce the amount of animal experiments. In this article, we present an innovative in vitro experimental design aimed to detect cardiotoxicity by obtaining a complete view of the induced mechanisms of a compound. By better reflecting the human in vivo conditions, we aim to find an in vitro model capable of reliably replacing animal models used within the field of toxicology and drug safety testing. First, instead of the regularly used monolayer cell cultures, a Human 3D cardiac microtissue (InSphero, SWL) model was used, which better resembles the in vivo heart ${ }^{[17-19]}$. This in vitro spheroid cell model contained approximately 4000 iPSCderived human cardiomyocytes and 1000 cardiac fibroblasts. Second, in contrast to using a relatively high dose to treat the cells, physiologically based pharmacokinetic (PBPK) modelling ${ }^{[20]}$ was included within the study design to better reflect the in vivo exposure. PBPK simulates the absorption, distribution, metabolism and excretion of a specific dose of DOX within the human body, therefore enabling us to predict the concentration to which a specific organ is exposed over time. The PBPK model was used to design a two weeks repetitive dosing profile in which microtissues were exposed to a decreasing dose over each day by means of 3 medium changes. The microtissues were exposed to either a therapeutic or a toxic dose of DOX using this PBPK-based repetitive dosing profile, and functional parameters for mitochondrial 
function and programmed cell death were assessed. Finally, by applying nextgeneration total RNA-sequencing to ribo-depleted RNA samples, we were able to analyze global changes in gene expression. By using this innovative experimental in vitro experimental design, we were able to successfully detect biological changes in the three main identified toxicity processes of DOX (cardiomyocyte dysfunction, mitochondrial dysfunction and cell death). 


\section{Methods}

\section{Samples}

The human 3D cardiac microtissue (InSphero, SWL) model was used, containing approximately 4000 iPSC-derived human cardiomyocytes (female Caucasian donor) and 1000 cardiac fibroblasts (male Caucasian donor) per microtissue. The microtissues were cultured in 3D Insight ${ }^{\mathrm{TM}}$ Human Cardiac Microtissue Maintenance Medium (InSphero, Cat \#CS-07-010-01).
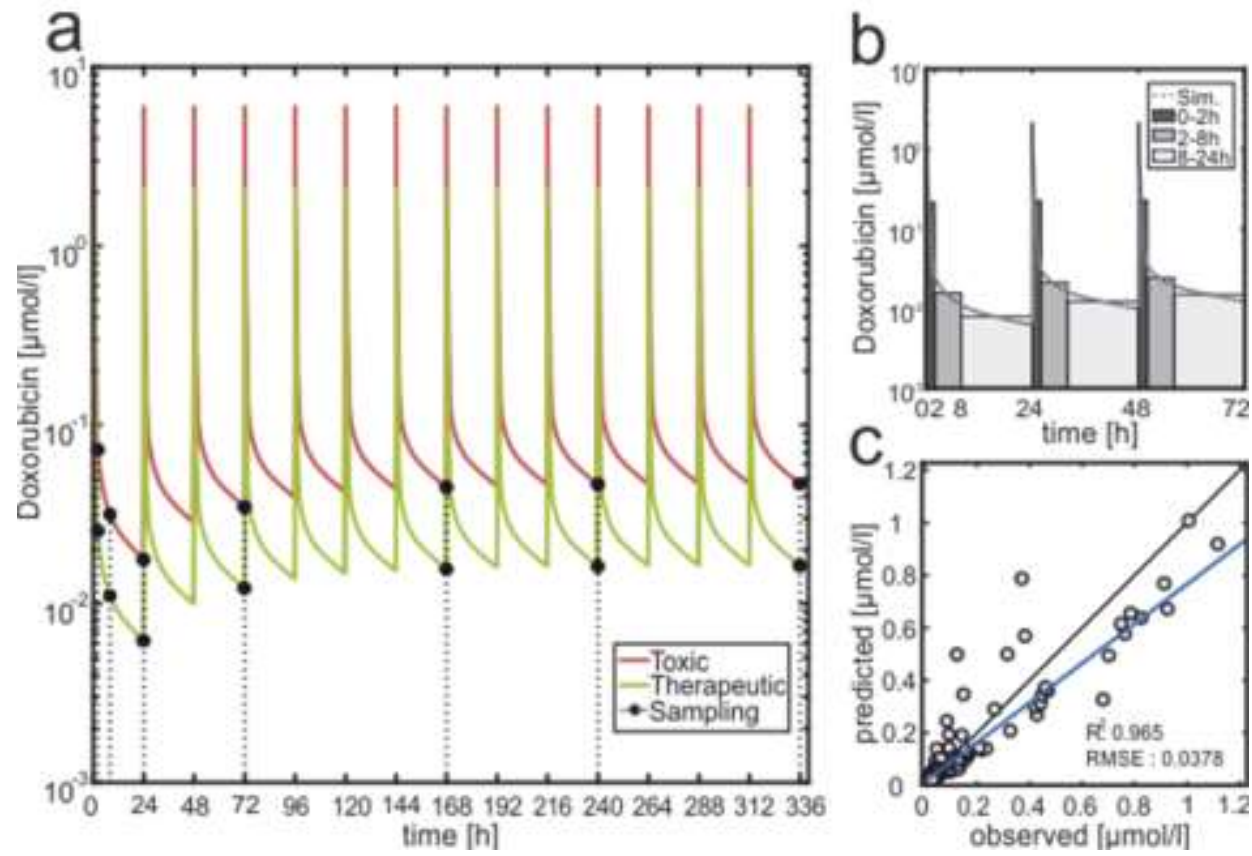

Figure 1: Doxorubicin PBPK model simulations. a) Simulations of DOX interstitial heart concentration profiles following a therapeutic (green) and toxic (red) once daily dosing regimen with experimental sampling points (black circles). b) Exemplary translation of PBPK simulations into discrete in vitro assay concentration with equal exposure, applied to cardiac microtissues for the first three days. c) Validation of PBPK model predicted DOX blood plasma concentrations with literature data ${ }^{[23]}$

\section{PBPK model establishment}

A PBPK model for DOX was established with the PBPK modelling software PK-Sim following a previously described workflow ${ }^{[21]}$. In particular, the model was used to quantify interstitial heart concentrations following administration of therapeutic and toxic drug doses. These concentration profiles were subsequently translated into 
discrete daily exposure profiles (Figure 1a, b). During model establishment, model predictions of blood plasma DOX concentrations were compared with literature data for parameter identification ${ }^{[22]}$. In a next step, the model was validated with further PK data4 (Figure 1c). Once daily therapeutic doses (0.415 mg*kg-1) were taken from literature while toxic doses $\left(1.18 \mathrm{mg}^{*} \mathrm{~kg}-1\right)$ were estimated based on in vitro doseresponse experiments. The toxic dose was estimated, such that the in vitro drug exposure (AUC) after 7 days resulting in a $20 \%$ loss of cell viability (IC20) was equal to the unbound drug exposure in the interstitial heart compartment of the PBPK model after a 7 day once daily drug administration regimen (Figure 1a).

\section{Exposure}

To accomplish the PBPK-based repetitive dosing profile described above, the medium of the microtissues was changed three times daily on working days with the PBPK-determined DOX concentrations (Sigma D1515, Cat \#25316-40-9). These were administered using stock solutions of the compound dissolved in DMSO. As control, microtissues exposed to similar end concentrations of DMSO were used. 7 time points $(2,8,24,72,168,240$ and 336 hours) were sampled in triplicate during the 2 weeks treatment period. For each sample, 36 microtissues were incubated separately, and subsequently pooled before RNA extraction in order to generate sufficient input material for NGS analysis.

\section{RNA sequencing \& Data processing}

From the exposed microtissues, total RNA was isolated using Qiagen AllPrep DNA/RNA/miRNA Universal Kit (Cat \#80224). The sample was depleted of ribosomal RNA using the Illumina RiboZero Gold kit (Cat \#MRZG12324) and prepared for sequencing using Lexogen SENSE total RNA library preparation kit (Cat \#009.96). After library preparation, the samples were sequenced on the HiSeq2500 (100bp pairedend). A pool of all DOX samples was sequenced on all 8 lanes of a flow cell. The same was done for the control samples. Control samples were sequenced with an average of 51.2 million raw reads, therapeutic DOX with 20.3 million reads and toxic DOX with 18.7 million. Because the sequencing reads still contained Lexogen adapter sequences, 
the first 12 bases of the 5'end of all reads were removed using Trimmomatic version $0.32^{[24]}$. The quality of the sequencing data was checked using FastQC version 0.11.3 ${ }^{[25]}$ before and after trimming.

\section{Gene expression analysis}

For each sample, sequencing reads were mapped to the GENCODE version 26 (GRCh38) - Ensembl 88 (Comprehensive gene annotation) using the Genedata Profiler $^{\mathrm{TM}}$ version 10.1, which incorporates the splice junction mapper STAR version 2.5.2b and Cufflinks based algorithms for transcript abundance estimation. The quality of the samples was assessed according to the amount of (mapped) reads, Cook's distance, hierarchical clustering, principal component analysis, and sample dispersion. 1 replicate of DOX toxic $240 \mathrm{~h}$ was excluded because of low read count. Differentially expressed genes (DEGs) were determined using DESeq2 version 1.14.1 ${ }^{[26]}$. For each specific time point, 2 comparisons were performed: 1) therapeutic dose vs DMSO control; 2) toxic dose vs DMSO control. Samples of all time points for both doses were normalized together and the contrast function was used to extract differentially expressed genes for each time point, ensuring comparability between time points. Genes were being considered as differentially expressed when $\mathrm{q}<0.05$.

\section{Biological interpretation}

For each dose and time point, lists of DEGs (depleted of ribosomal genes) were used as input for pathway over-representation analysis (ORA) using CPDB release $32^{[27]}$. The Reactome database version 61 was selected because it is curated, focuses on molecular mechanisms and includes pathway hierarchy ${ }^{[28]}$. Results from this analysis was visualized using Omix visualization version $1.9 .20^{[29]}$.

\section{Functional assessments: ATP measurement, Caspase $3 / 7$ assay and $0 x y g e n$}

\section{consumption}

For measurement of ATP, Promega's CellTiter Glo 3D (Cat \#G9683) was used according to manufacturer's protocol. In short, the microtissues were incubated for $30 \mathrm{~min}$ with luciferase reagent and the luminescence was measured. Apoptosis induction was measured using Promega's caspase-Glo $3 / 7$ assay (Cat \#G8092) 
according to manufacturer's protocol. Briefly, the Z-DEVD-aminoluciferin substrate is cleaved by caspase $3 / 7$, releasing a substrate for luciferase (aminoluciferin), resulting in measurable luminescence. Mitochondrial function after 2 hours and 7 days of DOX treatment was assessed by measurement of extracellular oxygen consumption using Luxcel's MitoXpress X Xtra Oxygen Consumption Assay (Cat \#MX-200) according to manufacturer's protocol. In short, oxygen quenches the MitoXpress ${ }^{\circledR}$ Xtra probe, making the measured fluorescent signal, inversely proportional to the oxygen concentration.

\section{Mitochondrial model}

The computational mitochondrial model ${ }^{[30]}$ for proteomics analysis was extended to evaluate the expression changes of genes functioning in mitochondria. The original model contained reactions of metabolites and involved protein complexes. For each protein complex represented in the model, the corresponding genes coding for each individual protein subunit were added. 


\section{Results}

In order to investigate mechanisms of DOX-induced toxicity, human 3D cardiac microtissues were exposed to DOX for two weeks with either a therapeutic dose ( $\left.D_{\text {THE }}\right)$ or a toxic dose $\left(D_{\text {TOX }}\right)$ using a PBPK based dosing profile. To this end, the medium of the microtissues was changed three times per workday with a decreasing dose. Samples were generated after $2,8,72,168,240$ and 336 hours of DOX exposure for both doses (Figure 2A).

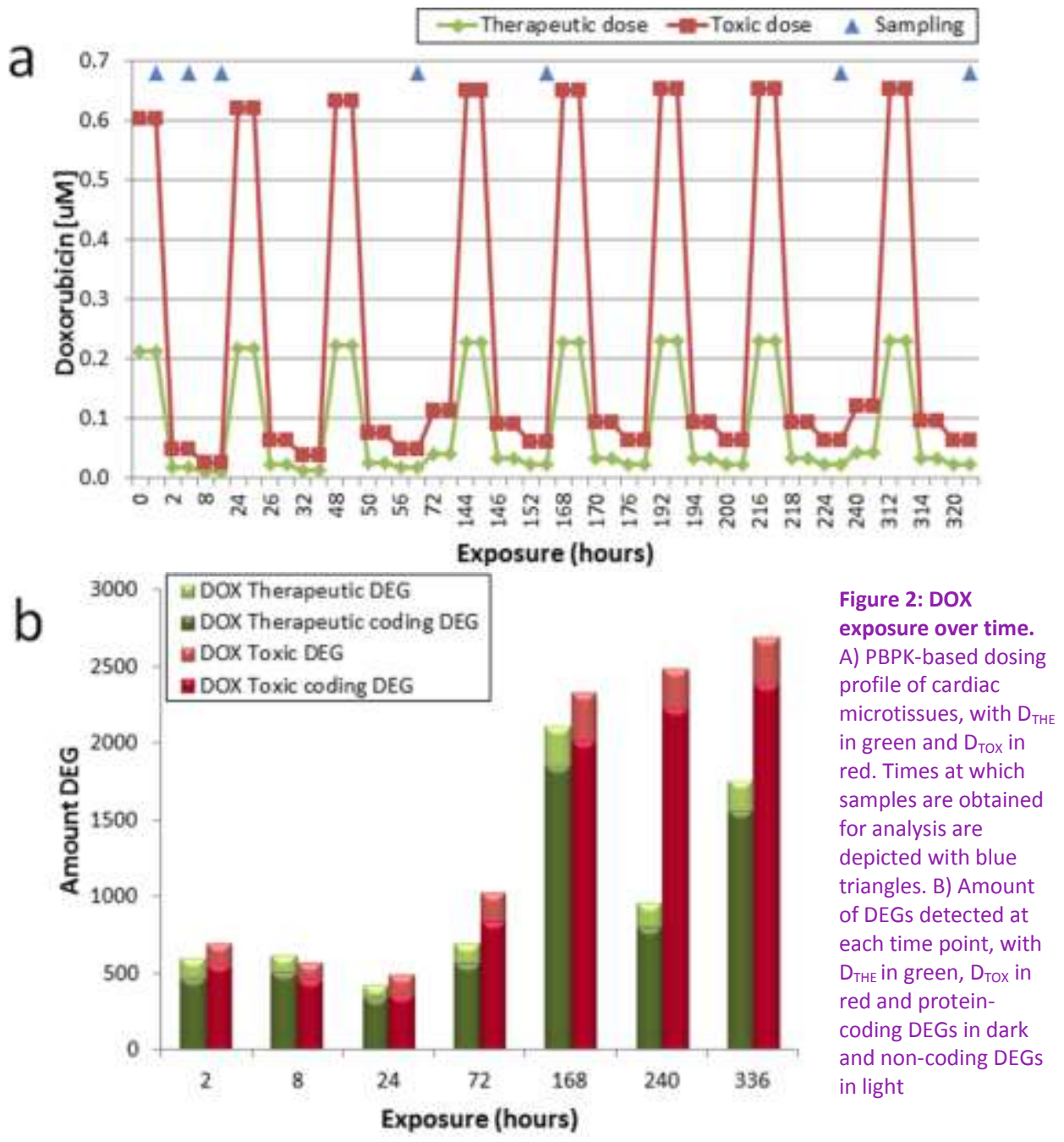


Molecular effects of DOX on the cardiac cell were investigated using RNAsequencing. The amount of differentially expressed genes (DEGs) increased over time (Figure 2B), except for a temporary decrease in DEGs at 240 hours of $\mathrm{D}_{\mathrm{THE}}$. Consistently across all time points, more DEGs were detected during exposure with $D_{\text {Tox }}$ than with $D_{\text {THE. }}$ Of these DEGs, $80 \%$ were protein-coding genes.

\section{Identifying known DOX pathways in vitro}

An overview of the effects of DOX on the whole cardiac cell was investigated to identify processes linked to DOX toxicity. This was done through pathway overrepresentation analysis (ORA). Overrepresented pathways were visualized in supplementary figure 2 . More significantly overrepresented pathways were identified for $D_{T H E}$ compared to $D_{\text {TOX }}$. This in combination with the higher amount of DEGs detected for $D_{\text {TOX }}$ compared to $D_{\text {THE }}$ indicates less specific cellular reactions for $D_{\text {TOX }}$. While therapeutic DOX exposure affects many cellular processes, the most significantly affected pathways throughout DOX treatment are "TCA cycle \& respiratory electron transport" ( $\left.q_{\text {median }} 2.92 \cdot 10^{-6}\right)$ and "Striated muscle contraction" $\left(q_{\text {median }} 9.3 \cdot 10^{-6}\right)$. These pathways can be related back to detrimental actions of DOX. Therefore, toxicity processes (cardiomyocyte dysfunction, mitochondrial dysfunction and cell death) were investigated in more detail (Figure 3), to determine whether our in vitro model expressed similar toxicity pathways as reported in literature for DOX.

Both DOX doses clearly induced "striated muscle contraction", the main pathway related to cardiomyocyte dysfunction. Both doses displayed also a decreasing amount of upregulated genes over time combined with an increase of downregulated genes. For $\mathrm{D}_{\text {TOX }}$, the amount of downregulated genes was always higher than of upregulated genes, while $D_{\text {THE }}$ displayed more upregulated genes until 240 hour of treatment. In "cellular senescence", more DEGs were observed for $D_{\text {TOX }}$ compared to $D_{\text {THE}}$, while the pathway was found significantly overrepresented for $D_{T H E}$ at more time points than for $D_{\text {TOx. }}$. This phenomenon is common in ORA and caused by the higher amount of DEGs and less specific cellular reactions of $D_{\text {TOx }}$. More striking were the differences between doses with respect to metabolic processes within the mitochondria. While $D_{\text {THE }}$ 
•.. Chapter 3: Bringing in vitrocloser to ín vivo •..

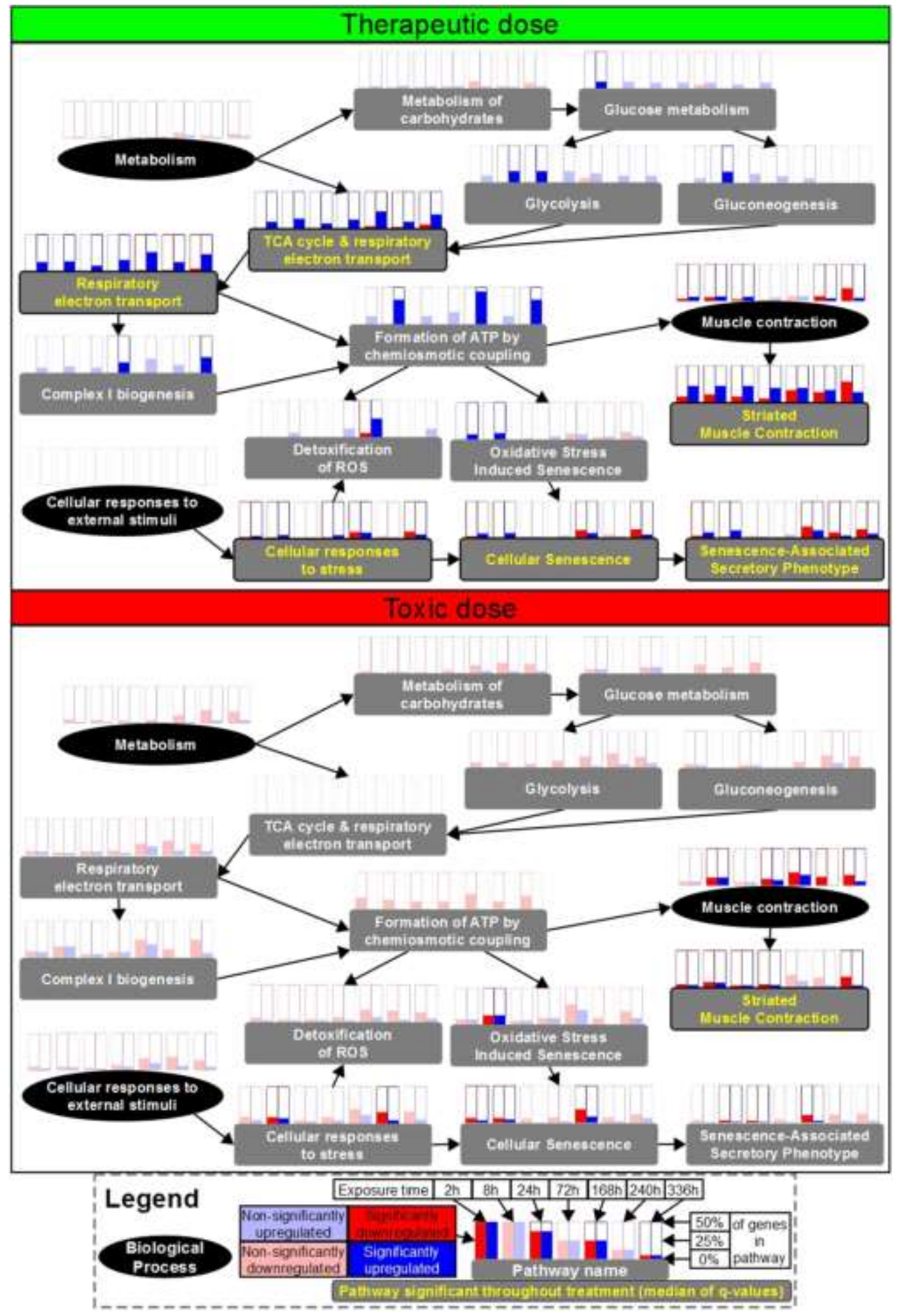


Figure 3: Specific toxicity processes affected by DOX. Visualization of overrepresented pathways, with $D_{\text {THE }}$ on the left and $D_{\text {TOX }}$ on the right. Black elipses identify clusters based on biological processes. Pathways (rectangles) were included when significantly overrepresented after DOX exposure minimally at 1 time point and at 1 dose, or in case the pathway was crucial for maintaining hierarchical links between the pathways. Coloured pathway names indicate pathways that were significantly overrepresented at the majority of time points (median of q-values $<0.05$ ). Bars on top of the pathway names depict up- and downregulation (blue and red resp.) per time point. The fill level corresponds to the percentage of DEGs detected (either up or down) in the pathway. The bars also depict if gene expression changes were significant at individual time points, with bright red and blue representing significant overrepresentation of the pathway at the specific time point, while faded colours are not significantly overrepresented at the given time

induced upregulations over time, $\mathrm{D}_{\text {TOX }}$ induced downregulations at the end of the DOX treatment period $(168 \mathrm{~h}-336 \mathrm{~h})$. Because mitochondria are involved in cell death and ATP generation necessary for cardiac contractile function, this dose-dependent difference in DOX-induced expressions of genes involved in mitochondrial processes may represent the underlying cause for differences in phenotypes for chronic or acute cardiotoxicity. Therefore, it is of interest to analyze mitochondrial dysfunction in more detail.

\section{Functional assessment of microtissue viability}

With respect to the ATP content of DOX-treated cells, the two analyzed doses (Figure $4 A$ ) were significantly different ( $p$-value 0.027 ) from each other. For $D_{\text {TOx }}$ ATP content decreases slightly until $72 \mathrm{~h}$, followed by a steep decrease in which ATP content is lower than the control. For $D_{T H E}$, a stable ATP content of approximately 20 $\mathrm{pmol} /$ microtissue is measured, except for a temporary drop at 240 hours of exposure. Between $24 \mathrm{~h}$ and $168 \mathrm{~h}$, ATP content is increased compared to the DMSO controls. Relating these results to the gene expression changes observed in the pathway "Formation of ATP by chemiosmotic coupling", indicates that increased gene expression is necessary for maintaining stable ATP content, while ATP content drops when no changes in gene expression are taking place. This suggests a decreased functionality and/or viability of the cardiac microtissues. Though cell death was not obviously detected on the gene level, this decreased viability was reflected from the caspase $3 / 7$ assay results performed at 2 and 168 hours of DOX exposure (Figure 4B). Here, caspase activity was more than twice as high compared to UNTR $\left(p_{2 h}=0.001\right.$; 
$\left.p_{168 h}=0.022\right)$ and $D_{T H E}\left(p_{2 h}=0.030 ; p_{168 h}=0.039\right.$. This difference in cell viability was also indirectly confirmed by the size of the microtissues, which visually decreased over time for $D_{\text {TOX }}$ but not for $D_{\text {THE }}$.

a
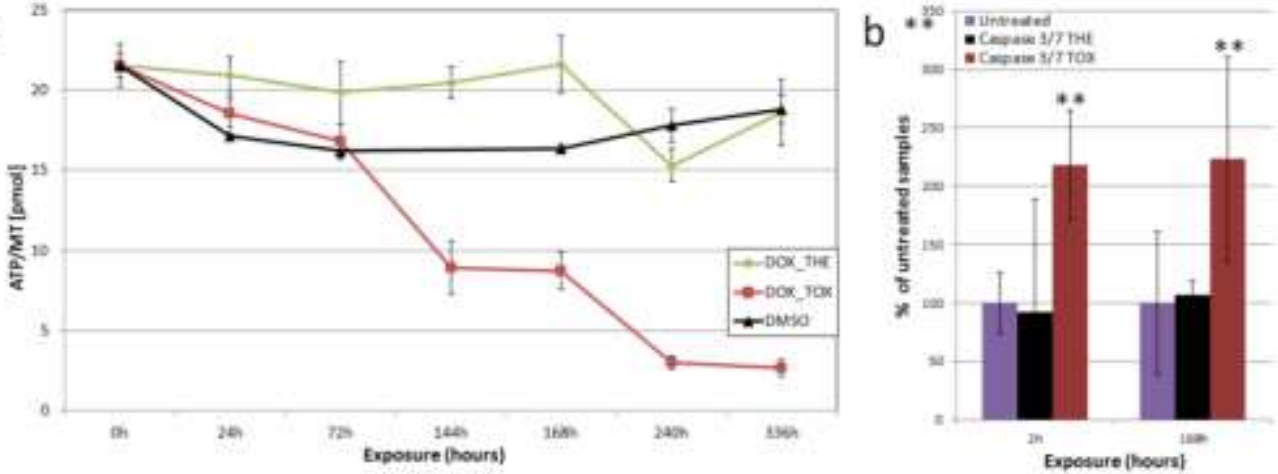

C

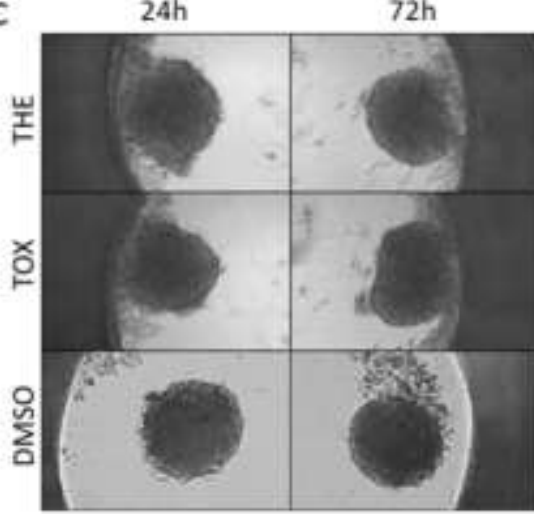

d

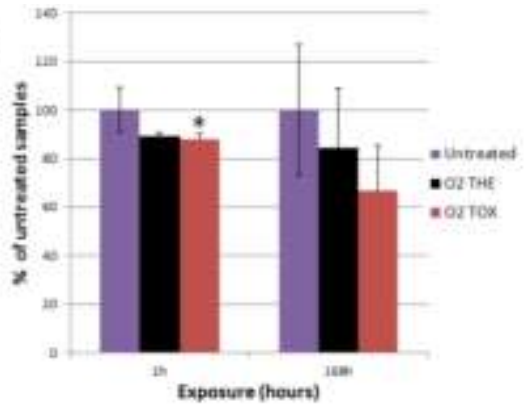

$168 \mathrm{~h}$

$240 h$

$336 \mathrm{~h}$
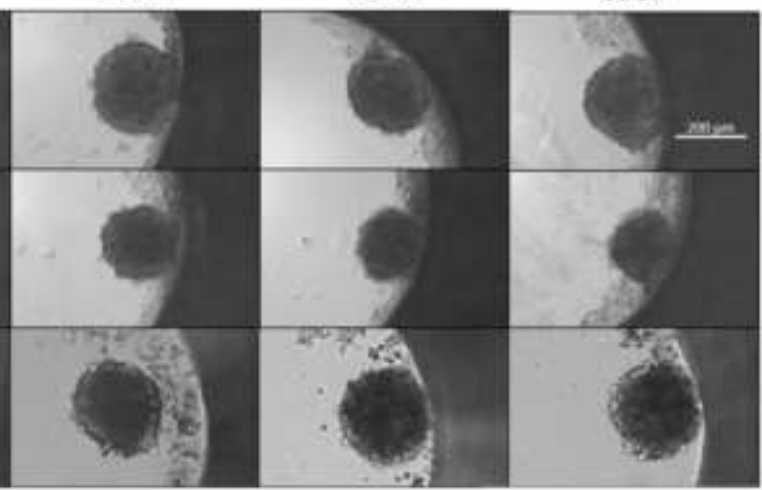

Figure 4: Functional assessment of microtissue viability.

A) Changes in ATP content of DOX exposed cardiac microtissues compared to DMSO control samples, with $D_{\text {THE }}$ in green, $D_{\text {TOX }}$ in red and DMSO control in black. B) Caspase activity

measurements at $2 \& 168$ hours of exposure compared to untreated microtissues. C) Example of a cardiac microtissue at each sampling time point to indicate microtissue size. D) Oxygen consumption measurements at $2 \& 168$ hours of exposure compared to untreated microtissues. * significantly different from control; ${ }^{* *}$ significantly different from control and $D_{\text {THE }}$ 
After 2 hours of DOX exposure, oxygen consumption was decreased by approximately $11 \%$ at both doses $\left(p_{\text {THE }}=0.061 ; p_{\text {TOX }}=0.048\right.$ ). After 168 hours of exposure, a difference oxygen consumption induced by the two doses was visible, with a decrease of $15 \%(p=0.369)$ for $D_{\text {THE }}$ and $33 \%$ for $D_{\text {TOX }}(p=0.064)$. While the decrease for $D_{\text {TOX }}$ can be partially caused by a lower amount of viable cells per microtissue, the decrease in oxygen consumption for $D_{\text {THE }}$ suggests decreased mitochondrial function.

\section{Mitochondrial effects of DOX exposure}

To elucidate the effects of DOX on genes functioning in mitochondria, DEGs were mapped onto the computational mitochondrial model ${ }^{[30]}$ adapted for transcriptomics data (Figure 5). As expected, the mitochondrial model showed major differences between the two doses. $D_{\text {THE }}$ displayed a majority of upregulated genes, while $D_{\text {TOx }}$ displayed more downregulations, especially at later time points.

\section{Detoxification of ROS}

Detoxification of ROS (bottom left of Figure 5A\&B) was activated to the same extent for both doses, though there are slight differences in the final step of ROS detoxification, in which $\mathrm{H}_{2} \mathrm{O}_{2}$ is converted to water. While for $\mathrm{D}_{\text {THE }}$ this reaction was mainly catalyzed by peroxiredoxin $(P R X)$, for $\mathrm{D}_{\text {TOX }}$ this reaction was carried out by both peroxiredoxin $(P R X)$ and glutathione peroxidase $(G P X)$. Overall, the upregulations found in these ROS detoxification genes indirectly indicated an increase in ROS formation in response to DOX. 
•. • Chapter 3: Bringing ín vitrocloser to ín vívo —. •

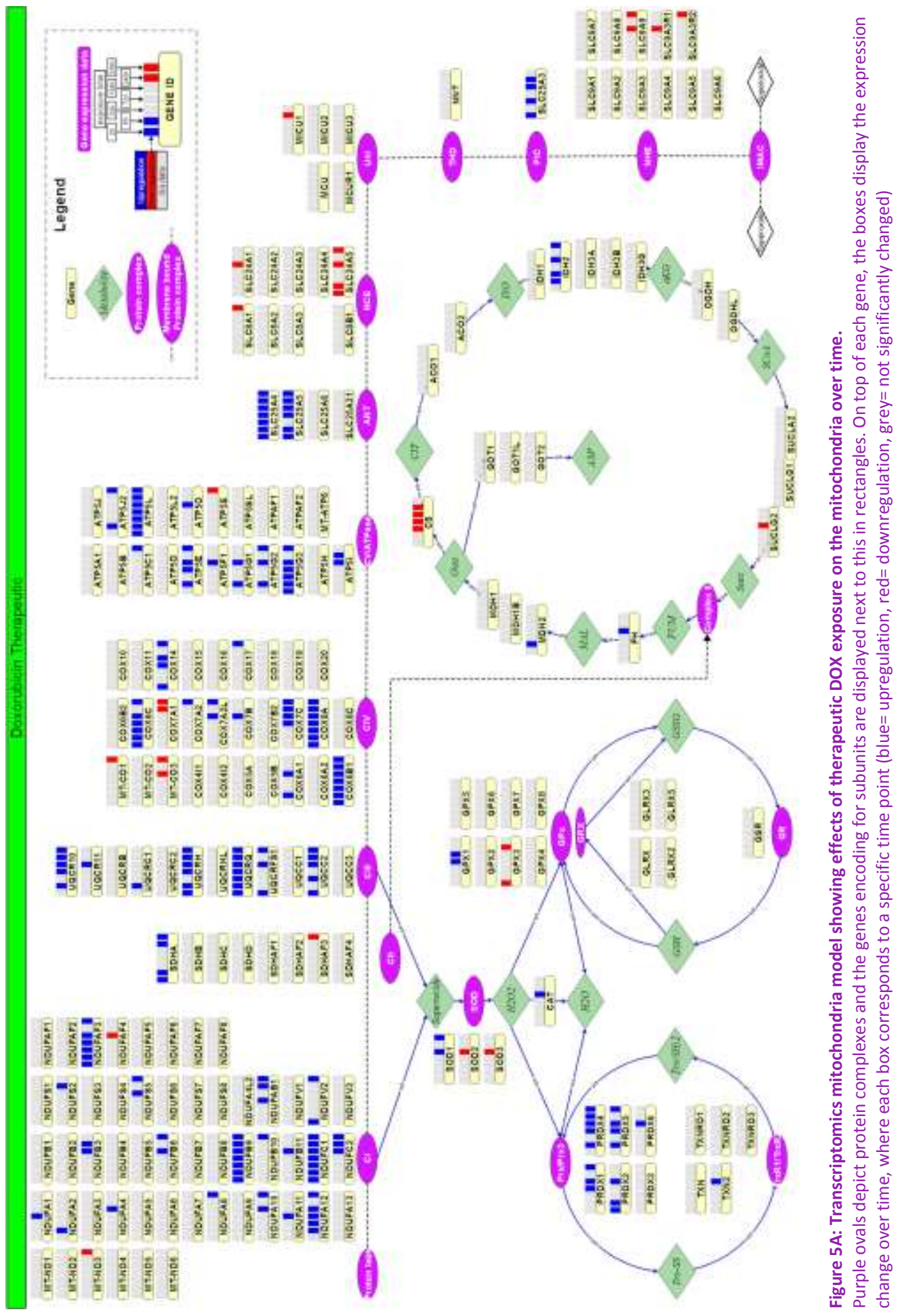




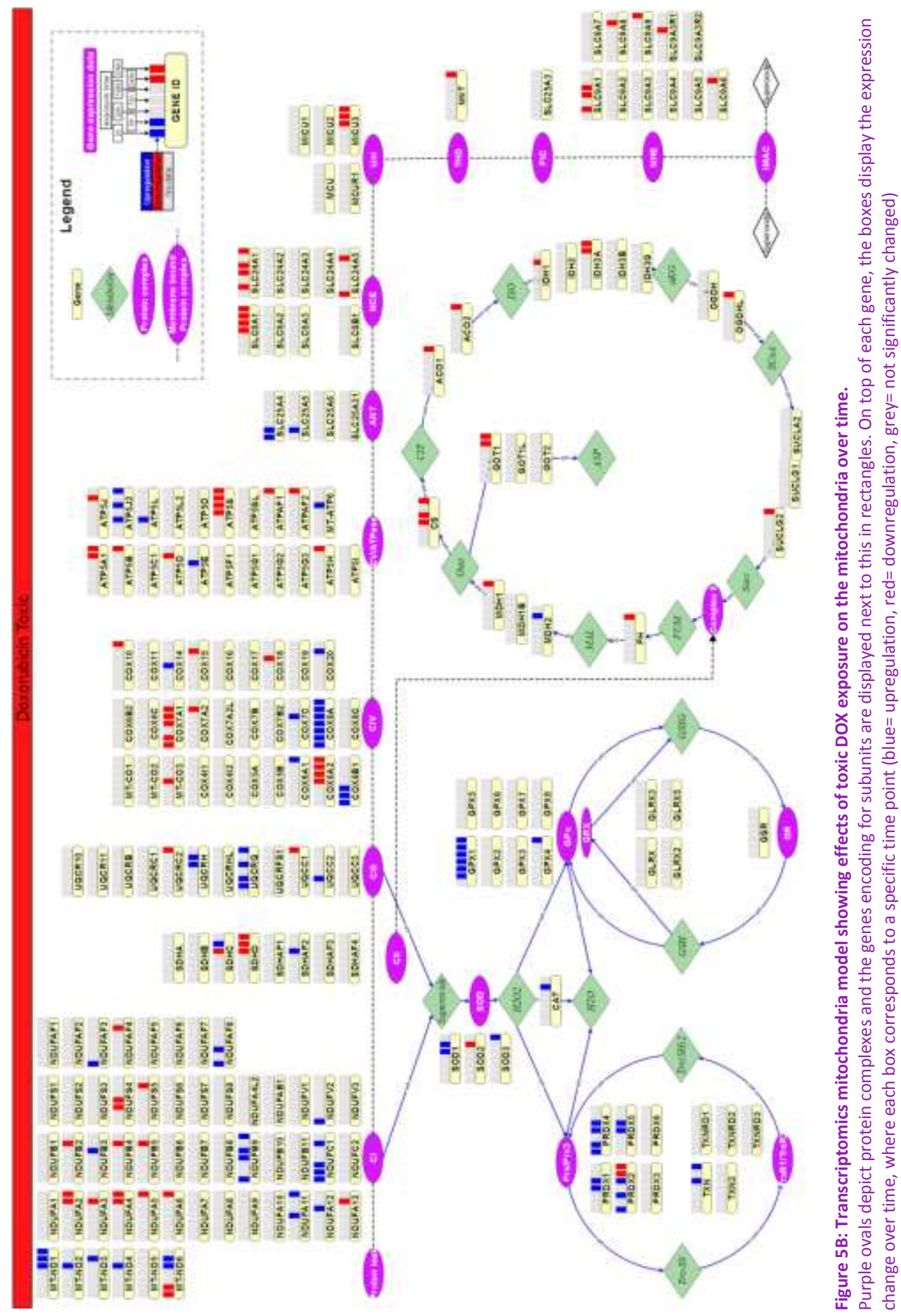




\section{Electron transport chain and formation of ATP}

Table 1: DOX effect on ETC complexes, overview of affected genes at minimally one time point

\begin{tabular}{|c|c|c|c|c|c|c|c|c|c|}
\hline \multirow[t]{2}{*}{ 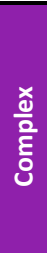 } & \multirow[t]{2}{*}{$\begin{array}{l}\text { Genes in } \\
\text { complex }\end{array}$} & \multicolumn{2}{|c|}{$\begin{array}{l}\text { Affected genes } \\
\text { (number / \% of } \\
\text { complex) }\end{array}$} & \multicolumn{2}{|c|}{$\begin{array}{c}\text { Genes } \\
\text { upregulated } \\
\text { (number / \% of } \\
\text { complex) }\end{array}$} & \multicolumn{2}{|c|}{$\begin{array}{c}\text { Genes } \\
\text { downregulated } \\
\text { (number / \% of } \\
\text { complex) }\end{array}$} & \multicolumn{2}{|c|}{$\begin{array}{c}\text { Genes significant }>4 \text { time } \\
\text { points } \\
\text { (number / \% of complex) }\end{array}$} \\
\hline & & $D_{\text {THE }}$ & $\mathrm{D}_{\text {TOX }}$ & $\mathbf{D}_{\mathrm{THE}}$ & $\mathbf{D}_{\text {THE }}$ & $\mathrm{D}_{\text {Tox }}$ & D $_{\text {Tox }}$ & $\mathrm{D}_{\mathrm{THE}}$ & $\mathbf{D}_{\text {TOX }}$ \\
\hline I & 53 & $\begin{array}{l}21 / \\
39.6\end{array}$ & $\begin{array}{l}25 / \\
47.2\end{array}$ & $\begin{array}{l}19 / \\
35.8\end{array}$ & $\begin{array}{l}2 / \\
3.8\end{array}$ & $\begin{array}{l}12 / \\
22.6\end{array}$ & $\begin{array}{l}12 / \\
22.6\end{array}$ & $\begin{array}{l}\text { NDUFA12, } \\
\text { NDUFB9, } \\
\text { NDUFC1, } \\
\text { NDUFAF3 }\end{array}$ & $\begin{array}{l}\text { MT-ND6, } \\
\text { NDUFB9, } \\
\text { NDUFC1 }\end{array}$ \\
\hline II & 8 & $\begin{array}{c}2 / \\
25.0\end{array}$ & $\begin{array}{c}3 / \\
37.5\end{array}$ & $\begin{array}{c}1 / \\
12.5\end{array}$ & $\begin{array}{c}1 / \\
12.5\end{array}$ & $\begin{array}{c}1 / \\
12.5\end{array}$ & $\begin{array}{c}1 / \\
12.5\end{array}$ & SDHA & - \\
\hline III & 12 & $\begin{array}{c}7 / \\
58.3\end{array}$ & $\begin{array}{c}5 / \\
41.7\end{array}$ & $\begin{array}{c}7 / \\
58.3\end{array}$ & 0 & $\begin{array}{c}2 / \\
16.7\end{array}$ & $\begin{array}{c}3 / \\
25.0\end{array}$ & $\begin{array}{l}\text { UQCR10, } \\
\text { UQCRH, } \\
\text { UQCRQ, } \\
\text { UQCC2 }\end{array}$ & UQCRQ \\
\hline IV & 29 & $\begin{array}{l}13 / \\
44.8\end{array}$ & $\begin{array}{l}13 / \\
44.8\end{array}$ & $\begin{array}{l}10 / \\
34.5\end{array}$ & $\begin{array}{c}3 / \\
10.3\end{array}$ & $\begin{array}{c}7 / \\
24.1\end{array}$ & $\begin{array}{c}6 / \\
20.7\end{array}$ & $\begin{array}{c}\text { cox6B1, } \\
\text { cox6C, } \\
\text { cox7C, } \\
\text { cox8A, } \\
\text { cox14 }\end{array}$ & $\begin{array}{c}\text { COX6A2, } \\
\text { COX7A1, } \\
\text { COX8A }\end{array}$ \\
\hline $\mathbf{v}$ & 21 & $\begin{array}{l}11 / \\
52.4\end{array}$ & $\begin{array}{l}12 / \\
57.1\end{array}$ & $\begin{array}{l}10 / \\
47.6\end{array}$ & $\begin{array}{l}1 / \\
4.8\end{array}$ & $\begin{array}{c}8 / \\
38.1\end{array}$ & $\begin{array}{c}4 / \\
19.0\end{array}$ & $\begin{array}{l}\text { ATP5E, } \\
\text { ATPG3, } \\
\text { ATP5L }\end{array}$ & ATP5S \\
\hline
\end{tabular}

Dose-dependent differences were most noticeable in the complexes of the ETC (top left of Figure $5 A \& B)$. The overall amount of genes affected (minimally at 1 time point) was similar between the doses, with complex I expressing the most DEGs. Because of the difference in amount of genes per complex, comparison in percentage of complex gave a better overview of the DOX effect (Table 1). Complex III was mostly affected for $D_{\text {THE }}$, while complex $V$ (also known as ATP synthase) was highly affected by both doses. Complex II was the least affected by DOX exposure. Dose-dependent differences were mostly observed through the direction of expression changes. Affected genes for $D_{\text {THE }}$ were mostly upregulated, while $D_{\text {TOX }}$ displayed an increase in downregulated genes, 
especially in later time points. Names of genes that were most highly affected by DOX exposure (significantly affected minimally at 4 time points) were also included in Table 1. Figure 6 depicts the log2 fold changes of these genes. Only gene expression changes of highly affected complex $\mathrm{V}$ genes were highly correlated to the changes observed in ATP content (Figure 4A), suggesting that the ATP level modification induced by DOX are directly monitored by the expression level of the genes involved in complex $\mathrm{V}$.

Overall, DOX-induced gene expression changes in the ETC were observed at both doses, though these changes indicate different underlying processes between the doses, discriminating between the phenotype of delayed chronic cardiac toxicity and acute cardiotoxicity.
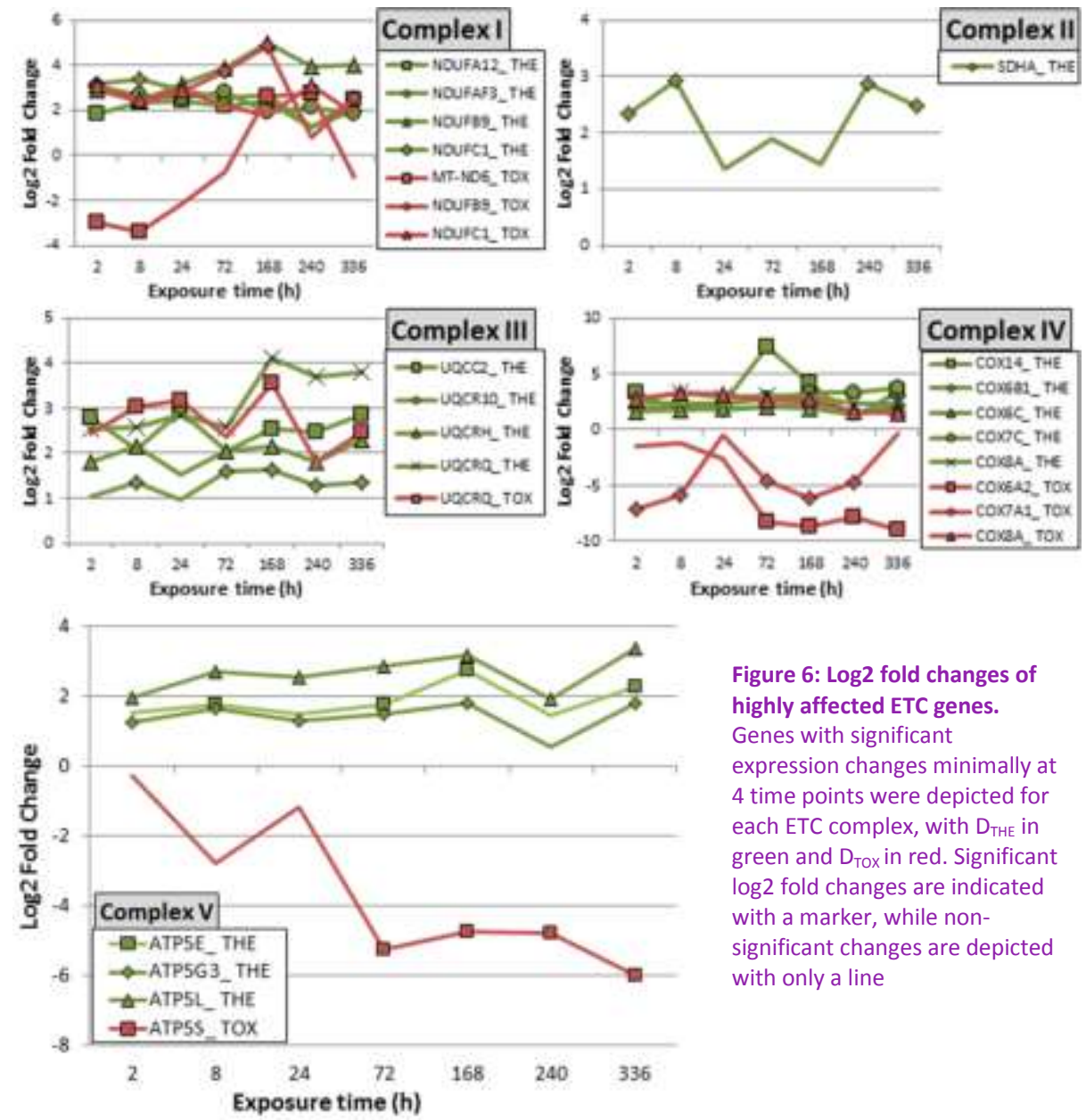

Figure 6: Log2 fold changes of highly affected ETC genes. Genes with significant expression changes minimally at 4 time points were depicted for each ETC complex, with $D_{\text {THE }}$ in green and $D_{\text {Tox }}$ in red. Significant log2 fold changes are indicated with a marker, while nonsignificant changes are depicted with only a line 


\section{Discussion}

The main objective of this study was to validate our novel in vitro experimental design for its use in predictive toxicological research. For this purpose, we analyzed cardiotoxic effects of doxorubicin (DOX). Our in vitro approach was designed to improve the resemblance to in vivo situations. This was achieved by using novel human 3D cardiac microtissues. This spheroid cell model contains a co-culture of iPSC-derived human cardiomyocytes and cardiac fibroblasts, better resembling the in vivo heart than monoculture monolayers. Furthermore, while standard toxicological research is being done with short time series and exposures at one specific dose, we applied a 2 week PBPK-based repetitive dosing profile that resembles in vivo drug concentrations in patients with one drug administration per day. The dose of DOX to which a patient's heart is exposed was calculated based on pharmacokinetic simulations of the absorption, distribution, metabolism and excretion of DOX. In vitro, the medium of the cardiac microtissues was changed three times daily with calculated DOX doses, resulting in the highest dose in the morning and the lowest dose in the evening of each working day during the 2 week exposure. The experimental design also included the exposure of two different dosing profiles, one based on therapeutic doses, as administered to a patient, and one based on a toxic dose. From these samples, RNA was extracted and sequenced to determine if this novel in vitro approach is able to retrieve the in vivo toxicity mechanisms known for DOX.

We used three detrimental actions of DOX which are known to occur in vivo (cardiomyocyte dysfunction, mitochondrial dysfunction and cell death) to determine that the in vitro approach was able to retrieve the in vivo toxicity pathways known for DOX ${ }^{[8,11,14,15]}$. Results from ORA indicated less specific cellular reactions for $D_{\text {TOx }}$, observed by the combination of more DEGs and less overrepresented pathways compared to $D_{\text {THE. }}$. Furthermore, ORA identified toxicity of DOX by gene expression changes in the pathways of "striated muscle contraction", "TCA cycle \& respiratory electron transport" and "cellular senescence". Gene expression changes in "striated muscle contraction" (cardiac function) and "cellular senescence" (cell death) displayed similar patterns, while mitochondrial processes ("TCA cycle \& respiratory electron transport") displayed significantly 
upregulated processes for $D_{\text {THE }}$ and non-significantly downregulated processes for $D_{\text {TOX. }} A$ central link between the toxicity pathways is the "formation of ATP by chemiosmotic coupling". Mitochondrial gene expression changes correlated with ATP measurements, in which $D_{\text {THE }}$ displays a relatively stable ATP content, while ATP content for $D_{\text {TOx }}$ decreased drastically over time. From this, we hypothesize that both doses induce toxicity, but the underlying processes between the doses are different, with its origin resulting from differences in mitochondrial functioning. Therapeutic doses reflect processes underlying the phenotype of delayed chronic cardiac toxicity, while toxic doses reflect processes of acute cardiotoxicity.

A more detailed analysis of mitochondrial dysfunction was done by investigating the 5 complexes of the ETC and activation of ROS detoxification processes. Mainly complex I, but also complex III, represent the sites of premature electron leakage to oxygen, resulting in the generation of ROS. Inefficiency of complex I can therefore result in increased ROS generation and oxidative stress. Furthermore, complex I is also a known interaction site for DOX, where it is reduced to form the oxidative semiquinone, which in turn, reduces oxygen to the highly reactive superoxide, returning DOX to its original state. Besides functioning as signaling molecules, ROS can damage proteins, nucleic acids and lipids in the cell. In order to prevent this, the cell is equipped with mechanisms to detoxify ROS. Gene expression changes indicated a similar increase in ROS detoxification between the doses. This confirms that ROS generation induced by DOX is indeed taking place in the in vitro model. Generated ROS is part of a positive feedback loop, in which more ROS results in more mitochondrial DNA (mtDNA) damage, leading to defects in the ETC complex, which results in increased ROS formation. Though uncertain whether this loop is initiated by generated ROS or if mtDNA damage is induced by DOX directly, this could explain the persistent and cumulative nature of DOX cardiotoxicity, even in the absence of the drug itself $^{[5]}$.

The detailed mitochondrial model also revealed major effects of DOX on the ETC and ATP production. Changes in this process differed significantly between the doses, with upregulations across time for $D_{T H E}$ and a majority of downregulations in later times for 
$D_{\text {Tox }}$. Most DEGs were detected in Complex I for both doses. Complex I is the starting point for transferring electrons form NADH through the ETC in order to generate ATP ${ }^{[31,32]}$. Also highly affected was Complex V (ATP synthase), which acts as a membrane channel for protons to be returned to the matrix. The flow back of protons gains the energy needed to convert ADP to ATP. The intermediate complexes of the ETC were also affected, though complex II only slightly. Because complex II is the only ETC complex that is completely encoded by the nucleus, and therefore less susceptible to mitochondrial $\operatorname{ROS}^{[14]}$, this strengthens the involvement of the previously noted positive feedback loop between ROS, mtDNA damage and defects in ETC complexes. This process is also supported by the expression profile in mitochondrial encoded genes for subunits of complex I (MT-ND genes), which displayed changes in toxic dose, but not in therapeutic dose.

The downregulations in gene expression at the later time points of toxic DOX exposure indicates the failure of ATP production through ETC. The decrease in ATP content of the microtissues was a continuous process throughout the exposure, a pattern that was also observed for ATP5S, the most affected gene in complex V. This gene, also known as factor $B$, is responsible for the energy transduction activity of the ATP synthase complex ${ }^{[33]}$. Though, it is unlikely that a defect of this one gene was solely responsible for the ATP decrease. Decreased effectiveness of the ETC may also be due to decreased oxygen availability, oxygen being consumed by DOX during ROS generation. Therefore, the ETC has less protons available to establish the proton gradient necessary for ATP production ${ }^{[14]}$. However, it seems more likely that the heterogeneity of DOX exposure causes malfunctioning cells in the outer layers of the microtissue to die, leaving a decreased amount of viable cells at the core to produce ATP. Because RNA-sequencing detects the mean gene expression of all living cells in the sample, this effect might not be significantly present to be detected in our data analysis. The detected decrease in gene expression at later time points therefore indicates adversity of the microtissue. This adversity was also observed in pathway analysis, where cellular processes are affected in less specific ways, indicating deregulation of the cell which may result in cell death. Though not observed by gene expression changes, cell death is observed in the cardiac microtissues at toxic doses of DOX through a decrease in microtissue size and increase of caspase 3/7 activity. 
In microtissues exposed to therapeutic doses, the DOX effect was also not equally distributed over the mitochondria present in the cells of the cardiac microtissue. Only a small amount of cells in the outer layer of the microtissue died, as could be seen from the small increase in caspase activity and a decrease in microtissue size that is hardly visible. However, an increase in gene expression of mitochondrial and ETC genes was necessary in order to maintain cellular ATP content, indicating a possible adaptation response in which less affected mitochondria compensate for highly affected mitochondria. However, this adaptation response may not be sufficient for maintaining cardiac function, because genes involved in striated muscle contraction were already showing decreasing expression level. Since the regenerative capacity of the heart is limited to ventricular remodelling, cumulative toxicity surpasses the point to which the organ can adapt, thereafter resulting in a delayed phenotype of heart failure, as is observed in patients with chronic cardiotoxicity after DOX treatment ${ }^{[4,15]}$.

\section{Conclusion}

Overall, these results confirm that our in vitro approach, based on 3D human microtissues exposed to an innovative 2 weeks PBPK dosing profile of DOX, is able to retrieve the known mechanisms of DOX toxicity. This new in vitro toxicity model can be extended to other 3D human microtissues, such as the already available liver model from primary hepatocytes.

The insights from the current study demonstrate the importance of applying physiologically relevant dosing profiles during toxicological research. Many studies on DOX effects have been performed with single and/or extremely high doses that do not reflect and may even obscure - the true mechanisms of toxicity. Therefore, controversy in published results must be treated cautiously because of the risk of oversimplification and mixture of the different mechanisms of acute and chronic effects ${ }^{[14,34]}$. Ultimately, we believe that this novel approach, better mimicking the human in vivo condition, could replace animal models within the field of toxicology and drug safety testing. 


\title{
Supplementary materials
}

\section{Supplementary figure 1: Visualization of the overrepresented DOX affected pathways.} ftp://web.tgx.unimaas.nl/mverheijen/Chapter3/Supplementary_figure1.docx

\author{
Supplementary data: DOX effects over time, full list of DEGs per time point \\ ftp://web.tgx.unimaas.nl/mverheijen/Chapter3/Supplementary_data.xlsx
}

\section{References}

1 Arcamone, F., Cassinelli, G., Di Marco, A. \& Gaetani, M. Patent application Farmitalia Research Laboratories. NSA 251 (1967).

2 Zhang, Y. W., Shi, J. J., Li, Y. J. \& Wei, L. Cardiomyocyte death in doxorubicin-induced cardiotoxicity. Arch Immunol Ther Ex 57, 435-445, doi:10.1007/s00005-009-0051-8 (2009).

3 Chatterjee, K., Zhang, J. Q., Honbo, N. \& Karliner, J. S. Doxorubicin Cardiomyopathy. Cardiology 115, 155162, doi:10.1159/000265166 (2010).

4 Chen, B., Peng, X. Y., Pentassuglia, L., Lim, C. C. \& Sawyer, D. B. Molecular and cellular mechanisms of anthracycline cardiotoxicity. Cardiovasc Toxicol 7, 114-121, doi:10.1007/s12012-007-0005-5 (2007).

5 Carvalho, F. S. et al. Doxorubicin-Induced Cardiotoxicity: From Bioenergetic Failure and Cell Death to Cardiomyopathy. Med Res Rev 34, 106-135, doi:10.1002/med.21280 (2014).

6 Kumar, S., Marfatia, R., Tannenbaum, S., Yang, C. \& Avelar, E. Doxorubicin-induced cardiomyopathy 17 years after chemotherapy. Tex Heart Inst J 39, 424-427 (2012).

7 Takemura, G. \& Fujiwara, H. Doxorubicin-induced cardiomyopathy from the cardiotoxic mechanisms to management. Prog Cardiovasc Dis 49, 330-352, doi:10.1016/j.pcad.2006.10.002 (2007).

8 Varga, Z. V., Ferdinandy, P., Liaudet, L. \& Pacher, P. Drug-induced mitochondrial dysfunction and cardiotoxicity. Am J Physiol Heart Circ Physiol 309, H1453-1467, doi:10.1152/ajpheart.00554.2015 (2015).

9 Edwardson, D. W. et al. Role of Drug Metabolism in the Cytotoxicity and Clinical Efficacy of Anthracyclines. Curr Drug Metab 16, 412-426 (2015).

10 Damiani, R. M. et al. Pathways of cardiac toxicity: comparison between chemotherapeutic drugs doxorubicin and mitoxantrone. Arch Toxicol 90, 2063-2076, doi:10.1007/s00204-016-1759-y (2016). 
11 Sorensen, J. C. et al. Mitochondria: Inadvertent targets in chemotherapy-induced skeletal muscle toxicity and wasting? Cancer Chemother Pharmacol 78, 673-683, doi:10.1007/s00280-016-3045-3 (2016).

12 Burridge, P. W. et al. Human induced pluripotent stem cell-derived cardiomyocytes recapitulate the predilection of breast cancer patients to doxorubicin-induced cardiotoxicity. Nat Med 22, 547-556, doi:10.1038/nm.4087 (2016).

13 Yang, F., Teves, S. S., Kemp, C. J. \& Henikoff, S. Doxorubicin, DNA torsion, and chromatin dynamics. Biochim Biophys Acta 1845, 84-89, doi:10.1016/j.bbcan.2013.12.002 (2014).

14 Berthiaume, J. M. \& Wallace, K. B. Adriamycin-induced oxidative mitochondrial cardiotoxicity. Cell Biol Toxicol 23, 15-25, doi:10.1007/s10565-006-0140-y (2007).

15 Tokarska-Schlattner, M., Zaugg, M., Zuppinger, C., Wallimann, T. \& Schlattner, U. New insights into doxorubicin-induced cardiotoxicity: The critical role of cellular energetics. J Mol Cell Cardiol 41, 389-405, doi:10.1016/j.yjmcc.2006.06.009 (2006).

16 Verheijen, M., Krauskopf, J., Kleinjans, J. C., de Kok, T. M. \& Caiment, F. Development and regulatory application of microRNA biomarkers. Biomark Med 9, 1137-1151, doi:10.2217/bmm.15.50 (2015).

17 Elliott, N. T. \& Yuan, F. A review of three-dimensional in vitro tissue models for drug discovery and transport studies. J Pharm Sci 100, 59-74, doi:10.1002/.jps.22257 (2011).

18 Achilli, T. M., Meyer, J. \& Morgan, J. R. Advances in the formation, use and understanding of multicellular spheroids. Expert Opin Biol Th 12, 1347-1360, doi:10.1517/14712598.2012.707181 (2012).

19 Zuppinger, C. 3D culture for cardiac cells. Bba-Mol Cell Res 1863, 1873-1881, doi:10.1016/j.bbamcr.2015.11.036 (2016).

20 Kuepfer, L. et al. A model-based assay design to reproduce in vivo patterns of acute drug-induced toxicity. Arch Toxicol, 1-3 (2017).

21 Kuepfer, L. et al. in Intracellular Delivery III: Market Entry Barriers of Nanomedicines (eds Aleš Prokop \& Volkmar Weissig) 363-374 (Springer International Publishing, 2016).

22 Speth, P. A., Linssen, P. C., Boezeman, J. B., Wessels, H. M. \& Haanen, C. Cellular and plasma adriamycin concentrations in long-term infusion therapy of leukemia patients. Cancer Chemother Pharmacol 20, 305-310 (1987).

23 Eksborg, S., Strandler, H.-S., Edsmyr, F., Näslund, I. \& Tahvanainen, P. Pharmacokinetic study of IV infusions of adriamycin. European Journal of Clinical Pharmacology 28, 205-212, doi:10.1007/bf00609693 (1985).

24 Bolger, A. M., Lohse, M. \& Usadel, B. Trimmomatic: a flexible trimmer for Illumina sequence data. Bioinformatics 30, 2114-2120, doi:10.1093/bioinformatics/btu170 (2014).

25 Andrews, S. FastQC: a quality control tool for high throughput sequence data. http://www.bioinformatics.babraham.ac.uk/projects/fastqc (2010).

26 Love, M. I., Huber, W. \& Anders, S. Moderated estimation of fold change and dispersion for RNA-seq data with DESeq2. Genome Biol 15, doi:ARTN 550 10.1186/s13059-014-0550-8 (2014).

27 Kamburov, A. et al. ConsensusPathDB: toward a more complete picture of cell biology. Nucleic Acids Res 39, D712-D717, doi:10.1093/nar/gkq1156 (2011).

28 Fabregat, A. et al. The Reactome pathway Knowledgebase. Nucleic Acids Res 44, D481-D487, doi:10.1093/nar/gkv1351 (2016).

29 Droste, P., Miebach, S., Niedenfuhr, S., Wiechert, W. \& Noh, K. Visualizing multi-omics data in metabolic networks with the software Omix: a case study. Biosystems 105, 154-161, doi:10.1016/j.biosystems.2011.04.003 (2011).

30 de Oliveira, B. \& Niederer, S. Computational models of doxorubicin mitochondria cardiotoxicity. J Pharmacol Tox Met 81, 349-349, doi:10.1016/j.vascn.2016.02.049 (2016).

31 Cooper, G. M. \& Hausman, R. E. The cell : a molecular approach. Sixth edition. edn, (Sinauer Associates, 2013).

32 Alberts, B. et al. Molecular biology of the cell. Sixth edition edn, (Garland Science, 2014).

33 Belogrudov, G. I. \& Hatefi, Y. Factor B and the mitochondrial ATP synthase complex. J Biol Chem 277, 6097-6103, doi:10.1074/jbc.M111256200 (2002).

34 Simunek, T. et al. Anthracycline-induced cardiotoxicity: Overview of studies examining the roles of oxidative stress and free cellular iron. Pharmacol Rep 61, 154-171 (2009). 


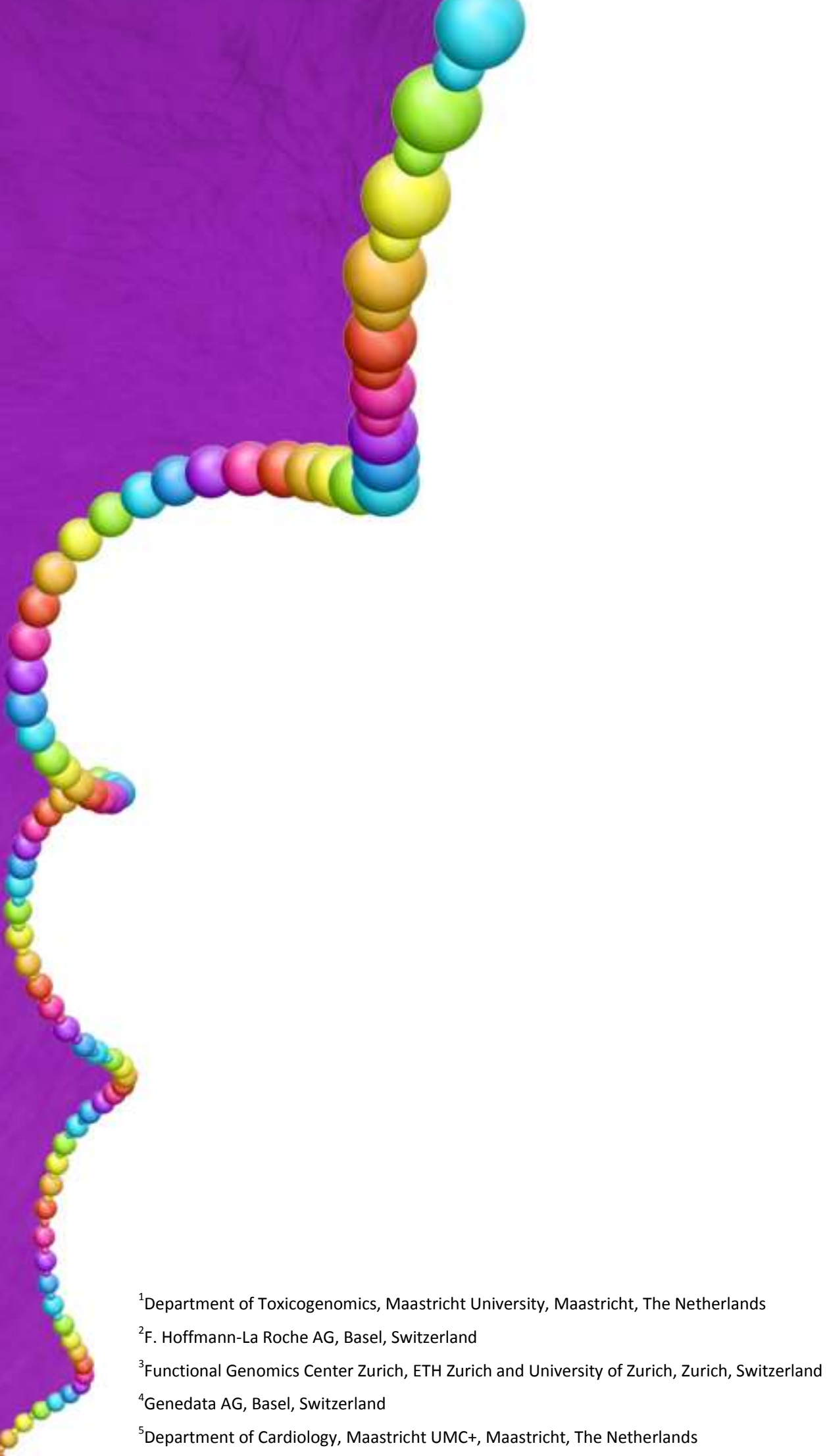




\section{Chapter 4 \\ Deeper than gene expression:}

Analyzing differential transcript usage of sarcomeric genes in anthracycline-induced cardiotoxicity

Marcha C.T. Verheijen ${ }^{1}$

Y. Schrooders ${ }^{1}$

O. Clayton ${ }^{2}$

R. Nudischer ${ }^{2}$

N. Selevsek ${ }^{3}$

R. Schlapbach ${ }^{3}$

H. Gmuender ${ }^{4}$

S. Gotta ${ }^{4}$

J. Merken ${ }^{5}$

S. Heymans ${ }^{5}$

J. Kleinjans ${ }^{1}$

F. Caiment $^{1}$

In preparation 


\section{Abstract}

The anthracyclines (ANTs) are widely used chemotherapeutic agents. While being effective in the fight against cancer, a major downside of ANT therapy is the occurrence of cardiotoxicity months or even years after the treatment has ended. While delayed chronic cardiotoxicity responds poorly to treatment, it can be prevented when treated within 3-6 months after the initial decrease in the left ventricular ejection fraction (LVEF). Therefore, early detection of ANT-induced cardiotoxicity is essential to prevent today's cancer patient from becoming tomorrow's cardiac patient. Because the ANTs doxorubicin, epirubicin and idarubicin are structurally highly similar and induce the same chronic cardiotoxic phenotype in patients, we hypothesize that dissecting the overlapping molecular mechanisms between these 3 anthracyclines enables us to identify potential biomarkers for early detection of ANT-induced cardiotoxicity. Therefore, we exposed 3D human cardiac microtissues in vitro to a single ANT for 2 weeks using a physiologically based pharmacokinetic (PBPK)-based repetitive dosing profile reflecting either a therapeutic dose or a toxic dose. By applying next-generation RNA-sequencing, we were able to analyze not only changes in gene expression, but also differential transcript usage (DTU; changed ratios of expressed transcripts of a gene) due to alternative splicing. We validated results obtained in vitro against human biopsies of heart failure patients to increase the clinical relevance.

With respect to sarcomere function - responsible for contraction of the heart two interesting gene targets were identified. First, titin - coding for a structural component of the sarcomere - displayed the most significantly changed DTU (FDR < $2.04 \cdot 10^{-31}$ ) in vitro and this correlated in vivo with increased left ventricular (LV) mass index $(p=0.002)$, decreased LVEF ( $p=0.018)$ and was the only transcript close to significant $(P=0.07)$ when comparing dilated vs undilated morphologies. The DTU entailed a switch from the TTN-206 transcript (encoding a truncated titin variant) to the TTN-212 transcript (encoding a full length protein) which may impact the stiffness of the sarcomere. Second, the changes in troponin- $T$ transcript usage detected in vitro and in vivo, are interesting because leakage of troponin proteins is already used to 
detect myocardial damage. The observed transcript-isoform switch from non-proteincoding transcripts to the TNNT2-207 transcript (encoding the cTnT3 protein isoform) might present a biomarker identifying ANT-related susceptibility to heart failure before myocardial damage occurs.

Overall, next to the discovery of two potential new biomarkers for early detection of ANT-induced cardiotoxicity, the current study demonstrates the added value of biological information gained from DTU analysis as compared to standard gene expression analysis. 


\section{Introduction}

The anthracyclines (ANTs) were amongst the first chemotherapeutic agents to be discovered in the 1960s and are still widely used for cancer treatment ${ }^{[1]}$. The most commonly used are doxorubicin (DOX), epirubicin (EPI) and idarubicin (IDA) ${ }^{[2]}$. While these ANTs are structurally highly similar, their small structural differences result in altered pharmacokinetic and pharmacodynamic properties. Compared to DOX, EPI has a reduced antitumor activity, a longer half-life and a higher body clearance. Furthermore, IDA has a higher cellular uptake and is more lipophilic ${ }^{[2,3]}$. The use of ANTs have contributed to increased cancer survival rates. Unfortunately, a major downside of ANT therapy is the occurrence of dose-dependent cardiotoxicity ${ }^{[1,2]}$. Therefore, maximum recommended cumulative doses (DOX: $400 \mathrm{mg} / \mathrm{m}^{2}$; EPI: $900 \mathrm{mg} / \mathrm{m}^{2}$; IDA: $150 \mathrm{mg} / \mathrm{m}^{2[2]}$ ) have been established. Still, ANT-induced cardiotoxicity can occur at any stage during the treatment (reversible acute cardiotoxic effects) or even months or years after the treatment has ended (irreversible delayed chronic cardiotoxic) $^{[4]}$. The chronic phenotype especially imposes health risks because of its poor response to treatments ${ }^{[5]}$, though it can be prevented when detected within 3-6 months after the LVEF (left ventricular ejection fraction) has started decreasing ${ }^{[6,7]}$. Features of cardiotoxicity include ventricular and contractile dysfunction, ultimately leading to dilated cardiomyopathy (DCM) and heart failure $(\mathrm{HF})^{[8,9]}$. While anti-tumor and cardiotoxic mechanisms have been extensively studied over the past 50 years, the exact mechanisms remain unclear ${ }^{[1,2]}$. The toxic process seems to be multifactorial, including generation of reactive oxygen species, mitochondrial dysfunction, topoisomerase II inhibition, DNA damage, cell death, myofibril instability, increased calcium sequestration, impaired compensatory mechanisms for stress and possibly other molecular processes ${ }^{[1-4]}$. It is essential to unravel the molecular processes of ANT-induced cardiotoxicity in order to develop methods for early detection and/or cardioprotective strategies so we can prevent today's cancer patient from becoming tomorrow's cardiac patient ${ }^{[2]}$. 
Since all three anthracyclines induce the same chronic cardiotoxic phenotype in patients, we hypothesize that dissecting the overlapping molecular mechanisms between the 3 anthracyclines will enable us to identify potential biomarkers for early detection of ANT-induced cardiotoxicity. To mimic the human in vivo conditions, cardiac 3D human microtissues were exposed to a single ANT (DOX, EPI or IDA) for two weeks using a physiologically based pharmacokinetic (PBPK)-based repetitive dosing profile reflecting either a therapeutic dose $\left(D_{\text {THE }}\right)$ or a toxic dose $\left(D_{T O X}\right)^{[10,11]}$. By applying next-generation total RNA-sequencing to ribo-depleted RNA samples, we were able to analyze not only changes in gene expression, but also differences due to alternative splicing. Splicing is a mechanism by which introns are removed form a precursor RNA to produce mature mRNAs that can be translated into a protein (providing the mRNA possesses an open reading frame). In the case of alternative splicing, a common RNA precursor can give raise to multiple transcript variants. A difference in transcript length can result from exon inclusion, exon skipping or retaining intron sequences ${ }^{[12]}$. While many of these transcripts are translated into proteins with related, distinct or opposite functions compared to the proteins resulting from the normally translated transcript ${ }^{[13]}$, they can also comprise non-coding variants. Furthermore, several transcripts can encode the same protein, though their translation rates may differ ${ }^{[14]}$. It is estimated that $92-94 \%$ of human genes are subjected to alternative splicing ${ }^{[13]}$. Due to differences in biological function of alternatively spliced transcripts, not only changes in gene expression, but also differential transcript usage (DTU) - which identifies changed ratios of expressed transcripts of a gene - can result in a disease phenotype $^{[12,15]}$. Therefore, we make use of technological advances of recent years to analyze DTU with the purpose of discovering new biomarkers for ANT-induced cardiotoxicity. In order to increase the clinical relevance, the in vitro observed DTUs were validated against DTUs observed in human biopsies of heart failure patients with or without prior ANT therapy. 


\section{Methods}

\section{In vivo Samples}

Cardiac biopsies were obtained from patients $(n=14)$ with decreased left ventricular function. Half of these patients have been treated in the past with DOX and/or EPI in combination with other none-ANT chemotherapeutic agents. These samples are labeled as cardiotoxic (CTX) because it is assumed that the left ventricular dysfunction is the result of the treatment. The other half of the patients displayed left ventricular dysfunction without having been treated with chemotherapy agents; these samples were used as the control group. For every CTX biopsy, there was a sample matched (SM) control biopsy available, matched on sex, age, BMI and LVEF. In total, 7 biopsy sets (CTX and its SM) were analyzed of which the patient information is listed in Table 1. Our study complies with the Declaration of Helsinki. The ethics committee/ institutional review board has approved the research protocol. Informed consent has been obtained from all the subjects.

\section{RNA isolation (in vivo)}

Tissue disruption of the biopsies was done by cryogenic grinding (mortar and pestle in liquid nitrogen). Thereafter, the Trizol/Qiazol protocol (Qiagen, Cat \#79306) was applied for RNA isolation ${ }^{[16]}$. Concentrations of RNA were measured the Qubit 2.0 Fluorometer system (Thermo Fisher Scientific, Waltham, MA USA) and the RNA quality was assessed using an Agilent 2100 Bio-analyzer (Agilent Technologies, Palo Alto, CA).

\section{In vitro Samples}

3D InSight ${ }^{\mathrm{TM}}$ Human Cardiac Microtissues (InSphero, SWL, for beta-testers), were cultured in 3D Insight ${ }^{\mathrm{TM}}$ Human Cardiac Microtissue Maintenance Medium (InSphero, SWL, Cat \#CS-07-010-01). The microtissues (MT) contained a co-culture of approximately 4000 iPSC-derived human cardiomyocytes from a female Caucasian donor with no known disease phenotype (Lot\# DMSO: CMC080112 ; DOX: CMC001523 ;

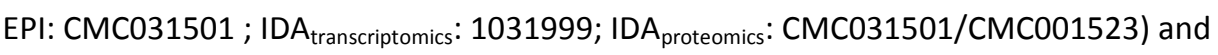
1000 cardiac fibroblasts from a 18 year old Caucasian male (Lot\# DMSO: HCF-av P9;

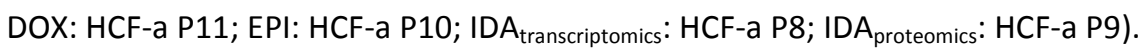


Table1: Detailed patient information

\begin{tabular}{|c|c|c|c|c|c|c|c|c|c|c|c|c|}
\hline Biopsy & $\begin{array}{l}\text { Descriptive } \\
\text { sample ID }\end{array}$ & Therapy & $\begin{array}{l}\frac{5}{5} \\
\text { dٓ }\end{array}$ & ڤ્ & $\sum_{\infty}$ & $\frac{\stackrel{Ð}{\frac{9}{4}}}{\frac{4}{3}}$ & 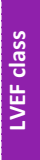 & 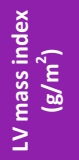 & 选 & 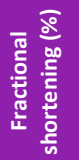 & 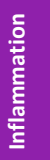 & Morphology \\
\hline CTX1 & CTX1_DE_B1_Dil & DOX, EPI, TAM & 1 & $\mathrm{~F}$ & 30 & 20 & 1 & 121 & 3 & 9.2 & Yes & Dilated \\
\hline SM1 & SM1_B1_Dil & None & 1 & $\mathrm{~F}$ & 29 & 32 & 1 & 112 & 3 & 25.4 & No & Dilated \\
\hline CTX2 & CTX2_D_B1_Dil & DOX, CYC, 5FU & 1 & $\mathrm{~F}$ & 23 & 33 & 1 & NM & 2 & 18.3 & No & Dilated \\
\hline SM2 & SM2_B1_Dil & None & 1 & $\mathrm{~F}$ & 23 & 19 & 1 & 156 & 4 & 8.6 & No & Dilated \\
\hline CTX3 & CTX3_D_B1_Un & $\begin{array}{l}\text { DOX, CYC, RTX, } \\
\text { VCR, PDN }\end{array}$ & 1 & M & 33 & 22 & 1 & 88 & 3 & 13.8 & No & Unaffected \\
\hline SM3 & SM3_B1_Dil & None & 1 & $M$ & 35 & 20 & 1 & 69 & 2 & 8.1 & No & Dilated \\
\hline CTX4 & CTX4_D_B2_Dil & $\begin{array}{l}\text { DOX, CYC, EVR, } \\
\text { EXE, TXT }\end{array}$ & 2 & $\mathrm{~F}$ & 26 & 23 & 1 & 106 & 2 & 10.6 & Yes & Dilated \\
\hline SM4 & SM4_B2_Dil & None & 2 & $\mathrm{~F}$ & 25 & 20 & 1 & 107 & 3 & 11.6 & No & Dilated \\
\hline CTX5 & CTX5_D_B2_Un & $\begin{array}{l}\text { DOX, CYC, VCR, } \\
\text { PDN }\end{array}$ & 2 & M & 23 & 65 & 2 & 74 & 2 & 35.3 & No & Unaffected \\
\hline SM5 & SM5_B2_Un & None & 2 & M & 23 & 47 & 2 & 75 & 2 & 24.5 & No & Unaffected \\
\hline CTX6 & CTX6_D_B2_Un & $\begin{array}{l}\text { DOX, CYC, RTX, } \\
\text { VCR, PDN }\end{array}$ & 2 & $\mathrm{~F}$ & 29 & 44 & 2 & 93 & 2 & 21.8 & Yes & Unaffected \\
\hline SM6 & SM6_B2_Dil & None & 2 & $\mathrm{~F}$ & 29 & 20 & 1 & 98 & 2 & 25.9 & Yes & Dilated \\
\hline CTX7 & CTX7_E_B2_Dil & EPI, DOC & 2 & $F$ & 29 & 34 & 1 & 139 & 2 & 16.4 & Yes & Dilated \\
\hline SM7 & SM7_B2_Un & None & 2 & $\mathrm{~F}$ & 27 & 39 & 2 & 81 & 1 & 22.0 & No & Unaffected \\
\hline
\end{tabular}

\section{PBPK model (in vitro)}

Using a previously described workflow ${ }^{[10,17]}$, the PBPK model for each ANT was obtained. In short, interstitial heart concentrations were quantified following administration of therapeutic and toxic drug doses. Daily therapeutic doses were based on literature, while daily toxic doses were gained from IC20 values after 7 days of exposure (determined through in vitro dose-response experiments).

\section{ANT treatment (in vitro)}

The above described PBPK-based repetitive dosing profile was realized through three medium changes per workday (highest daily dose $\rightarrow$ medium daily dose $\rightarrow$ lowest daily dose). In weekends, medium was not changed, but cells were exposed to the average dose per hour. ANTs (DOX, EPI or IDA) were added to the culture medium 
using stock solutions of the compound dissolved in DMSO. Due to a decreasing dose over the day, DMSO concentrations also decreased, which were mimicked in the control samples. The 2 week exposure included 7 sampling time points $(2,8,24,72$, 168, 240 and 336h), which were sampled in triplicate (21 samples per condition; in total $5 \times 21=147$ ). In order to obtain sufficient material, every sample contained 36 microtissues (incubated separately) that were pooled before isolation of RNA using the AllPrep DNA/RNA/miRNA Universal Kit (Qiagen, Cat \#80224) and 18 microtissues (incubated separately) for isolation of protein using freeze-thaw cycles followed by centrifugation.

\section{ATP measurement (in vitro)}

ATP content of the microtissues was measured using Promega's CellTiter Glo 3D (Cat \#G9683) according to manufacturer's protocol, in which luminescence was measured after 30 min incubation with the luciferase reagent.

\section{RNA sequencing (in vitro \& in vivo)}

RNA was depleted of ribosomal RNA using the Ribo-Zero Gold rRNA Removal kit (Human/Mouse/Rat, Illumina ${ }^{\circledR}$, Cat \#MRZG12324) and prepared for sequencing using the SENSE total RNA library preparation kit (Lexogen ,Cat \#009.96). Before sequencing on the HiSeq2000, the quality and quantity of the libraries was assessed on Agilent 4200 TapeStation and by Qubit ${ }^{\mathrm{TM}}$ respectively.

A pool of all samples per compound (DOX, EPI, IDA or DMSO) was sequenced per flow cell. Furthermore, human biopsy samples were sequenced in 2 separate batches. The average sequencing depth (raw reads/sample) was 51.2 million for DMSO, 20.3 million reads for therapeutic DOX, 18.7 million for toxic DOX, 25.3 million reads for therapeutic EPI, 20.7 million for toxic EPI, 24.2 million reads for therapeutic IDA, 21.9 million for toxic IDA, 20.5 million reads for cardiotoxic biopsies batch 1, 25.7 million for sample matched biopsies batch 1. 62.2 million reads for cardiotoxic biopsies batch 2 and 68.1 million for sample matched biopsies batch 2 .

The sequencer generated files for every lane in which the sample was measured. After concatenating the lanes, two files per sample remained, one containing left reads 
(R1) and one containing right reads (R2). The reads still contained Lexogen adapter sequences (first 12 bases of all reads), which were removed using Trimmomatic ${ }^{[18]}$ (v.0.32). Samples were excluded from analysis in cases of poor sequencing quality (assed by FastQC version $0.11 .3^{[19]}$ ), due to low read counts $(<1,000,000$ reads, which was the case for:DOX_TOX_240_2, IDA_THE_240_3 \& IDA_TOX_336_2) and when they were classified as an outlier (assessed through principal component analysis, which was the case for EPI_TOX_008_2).

\section{Obtaining transcript read counts and usage percentages}

(in vitro \& in vivo)

The obtained sequencing reads were aligned to the Ensembl reference genome (Genome Reference Consortium Human Build 38, GRCh38.p7) using RSEM ${ }^{[20]}$ (v.1.2.28) with the paired-end and Bowtie ${ }^{[21]}$ (v.2.2.6) option. This resulted in files containing read counts per gene and per transcript. Furthermore, for each gene the usage of each transcript were reported in percentage.

\section{Differential gene expression (in vitro)}

The DESeq2 ${ }^{[22]} \mathrm{R}$ package (v.1.16.1) was used to determine differentially expressed genes (DEGs; FDR <0.05). For each ANT, samples of all time points and both doses were normalized together. Thereafter, the DESeq2 design was set according to the exposure dose, either therapeutic vs DMSO or toxic vs DMSO. Furthermore, minimum count was set to an average read count of one across all samples. Other settings were kept to default. Finally, remaining ribosomal genes were filtered out of the dataset to ensure complete ribosomal depletion of the data.

\section{Differential transcript usage (in vitro \& in vivo)}

Differential transcript usage (DTU; FDR $<0.01$ and change $>15 \%$ ) was determined by ANOVA testing, comparing transcript usage (\%) of ANT vs DMSO. Furthermore, correlation of DTU with specific parameters of the biopsies (LVEF, LV mass index, NYHA class, Fractional shortening or morphology) was determined using two-sided T-tests. 


\section{Proteomics measurement (in vitro)}

Proteins were measured using mass spectrometry as described in chapter 2 . In short, after protein isolation, samples were diluted to a final concentration below 2M. Next, trypsin was used for protein digestion and peptide clean-up was done using Sep-Pak tC18 cartridges (Waters) according to the manufacturer's instructions. Before measurement on a Orbitrap Fusion mass spectrometer (Thermo Fisher Scientific) coupled to a NanoLC-2D HPLC system (Eksigent), peptides were dried using a vacuum centrifuge.

\section{Proteomics data-analysis (in vitro)}

The Genedata Expressionist software (v.11.0) was used to process raw MS data. First, data is subjected to noise reduction and normalization. Thereafter, LC-MS peaks were detected and their properties calculated $(\mathrm{m} / \mathrm{z}$ and RT boundaries, $\mathrm{m} / \mathrm{z}$ and RT center values, intensity). Annotation by using Mascot 2.6 was performed on individual MS/MS spectra. Mascot 2.6 parameters were set to: 10.0 ppm Peptide Tolerance, 0.50 Da MS/MS Tolerance, 2 Max Missed Cleavages and the Uniprot Swiss-Prot 29062016 database with Homo sapiens (human) Taxonomy. Rank 1 hits, filtered at 5\% False Discovery Rate (FDR), were accepted as valid. MS/MS spectra that could not be associated to any correctly featured cluster are discarded. Protein interference was used to group peak clusters and was based on peptide and protein annotations. Redundant proteins were ignored according to the Occam's razor principle, and at least 2 peptides were required for a positive protein identification (shared peptides were ignored). The $\mathrm{Hi} 3$ method was used to compute protein intensities. In the calculations, a maximum of the top 3 peptides per protein (based on the average intensity across samples) was used. Values were consolidated into a single peptide intensity when multiple charges $(2+, 3+, 4+)$ and modification states (Carbamidomethyl (C), Deamidated (NQ) or Oxidation (M)) were identified for the same peptide. For each ANT, $\log 2$ transformed data of each sample were shifted to the median of the medians of all samples (e.g. all DOX samples + all DMSO samples). Finally, determination of differentially expressed proteins (DEPs) was done using 2-sided T-tests. 


\section{Results \& Discussion}

In order to obtain a list of the

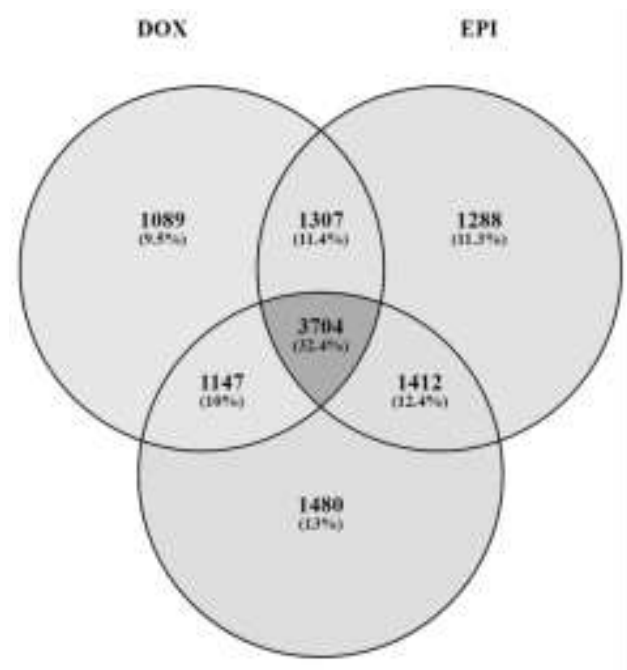

(ORA) was done using ConsensusPathDB

$(\mathrm{CPDB})^{[24]}$, thereby selecting the KEGG

database (curated database focused on

IDA

Figure 1: Venn diagram showing commonality between the ANTs disease processes) ${ }^{[25]}$. Significantly overrepresented pathways (q-value $<0.05$, Table $2 \&$ Supplementary Table 1) contained 5 pathways directly related to cardiac function: 1) Hypertrophic cardiomyopathy (HCM), 2) Dilated cardiomyopathy (DCM), 3) Cardiac muscle contraction, 4) Adrenergic (AR) signaling in cardiomyocytes and 5) Arrhythmogenic right ventricular cardiomyopathy (ARVC).

Table 2: ORA results of pathways directly related to cardiac function

\begin{tabular}{|lcc|c|}
\hline Pathway name & set size & candidates & q-value \\
\hline Cardiac muscle contraction & 78 & $37(47.4 \%)$ & $3.50 \mathrm{E}-07$ \\
\hline Hypertrophic cardiomyopathy (HCM) & 83 & $38(45.8 \%)$ & $3.50 \mathrm{E}-07$ \\
\hline Dilated cardiomyopathy (DCM) & 90 & $38(42.7 \%)$ & $2.67 \mathrm{E}-06$ \\
\hline Adrenergic signaling in cardiomyocytes & 149 & $54(36.2 \%)$ & $4.30 \mathrm{E}-06$ \\
\hline Arrhythmogenic right ventricular cardiomyopathy (ARVC) & 72 & $26(36.1 \%)$ & $2.36 \mathrm{E}-03$ \\
\hline
\end{tabular}

Because these pathways are highly related, DEGs functioning in these pathways overlapped. Therefore, all genes involved in these 5 processes were extracted from the KEGG database using the CPDB website to create an integrative pathway of heart 


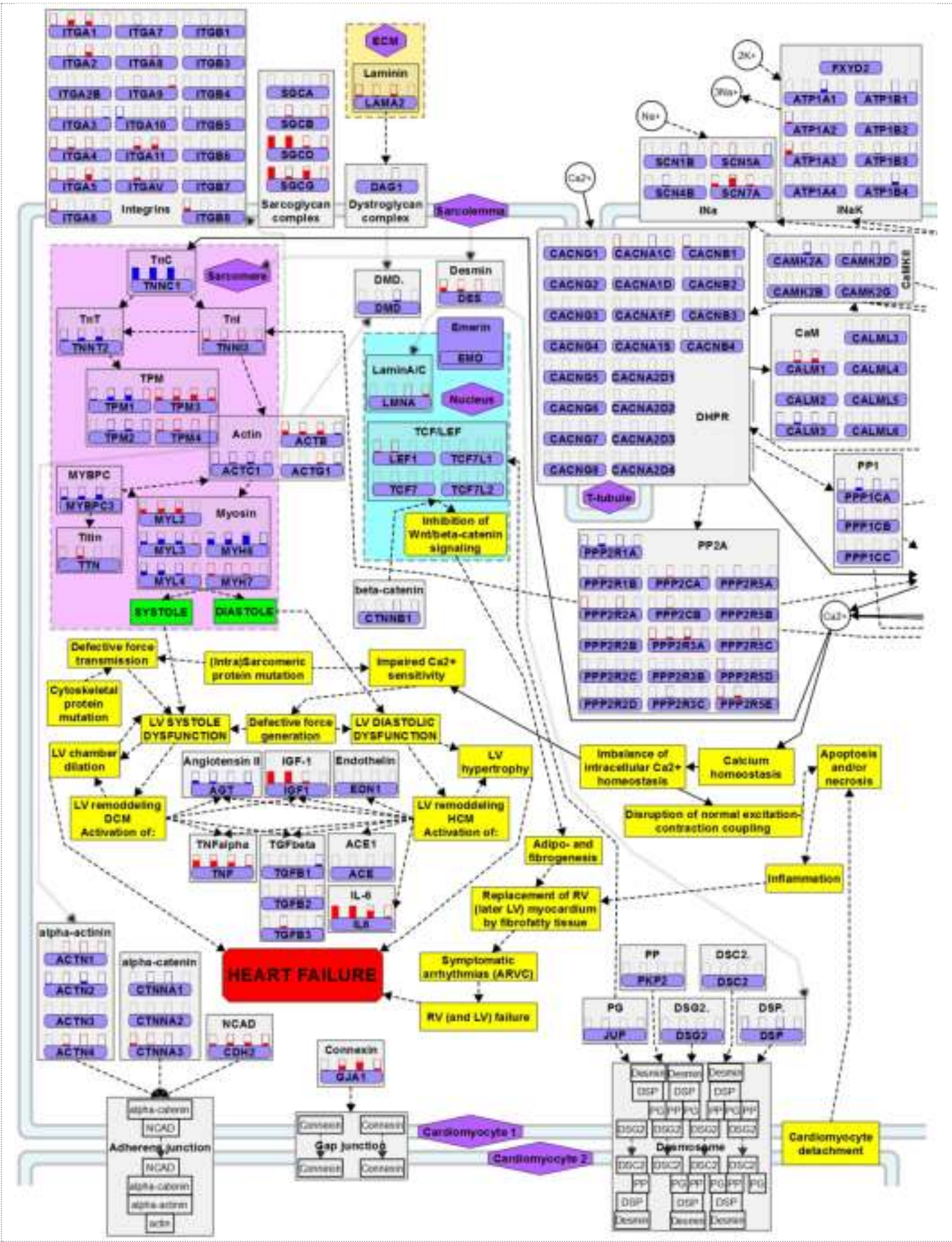

Figure 2: Heart failure model depicting gene expression changes after therapeutic ANT exposure. The model was made by merging 5 cardiac KEGG pathways. From each pathway, involved proteins obtained. Proteins localized in the nucleus were grouped in the blue area, mitochondria in the orange area and sarcomere in the pink area. Each protein is depicted as a box, with inside the gene(s) coding $\rightarrow$ continues on next page $\rightarrow$ 


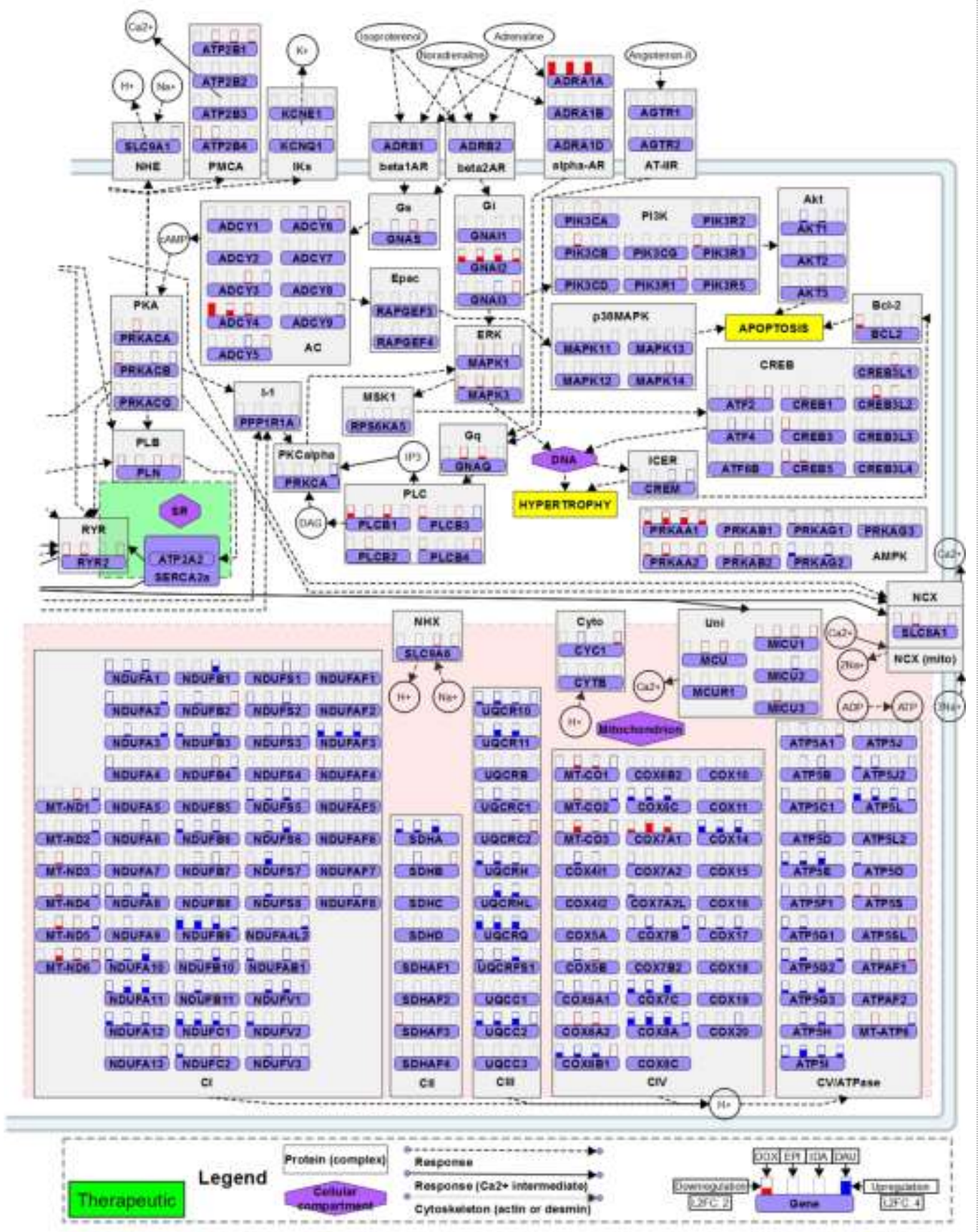

Figure 2 (continued): for them depicted in purple. Above every gene, there are bars indicating the expression changes (average log2FC of the 7 measured time points; red = downregulation; blue = upregulation; fill level of bars indicates magnitude of change based on the absolute log 2 fold change, completely filled bar $=\log 2 F C \geq 5$ ) for a single exposure. From left to right, the first bar corresponds to DOX exposure, the second to EPI, the third to IDA and the fourth DAU (excluded from study) 
failure (Figure 2, next page). Plotting the ANT gene expression data onto this model indicated high similarity between the ANTs, thus supporting our hypothesis. The heart failure model identified effects in the mitochondria and the sarcomere (Therapeutic dose: Figure 2, Toxic dose: supplementary Figure 1B). The main function of mitochondria is to supply ATP necessary for cellular processes. Functional ATP measurements (Figure 3) revealed higher ATP content for the therapeutic dose compared to the toxic dose (of the same compound at the same time), with the exception of IDA at $72 \mathrm{~h}$ of exposure. Dose-dependent differences become visible after $168 \mathrm{~h}$ of exposure, while before $72 \mathrm{~h}$ ATP curves of the doses are still intertwined. Microtissues exposed to therapeutic doses - leading to chronic toxicity in vivo did not change in ATP values across time, while in microtissues exposed to toxic doses resulting in acute toxicity in vivo - a clear decrease in ATP content was observed. This implies a difference in underlying cellular processes between the acute and chronic phenotypes. While acute toxicity appears to be related to a decrease in ATP content (which may be due to mitochondrial dysfunction), this does not seem the case for chronic toxicity ${ }^{[10]}$. This observation is in concordance with literature reporting impairment of mitochondrial bioenergetics by ANTs ${ }^{[5]}$ and a reduction of acute myocardial damage when patients are treated with dexrazoxane (a free-radical scavenger) prior to ANT exposure ${ }^{[4]}$. Because chronic cardiotoxicity constitutes the most severe health risks for ANT-treated cancer survivors, we limit our analysis to effects induced by the therapeutic dose. The sarcomere facilitates contraction and is highly important for heart function. Alterations in this compartment due to genetic mutations are known causes of familial HCM and DCM ${ }^{[26]}$. Therefore, this cellular compartment was investigated in further detail for discovery of interesting targets possibly representing biomarkers for ANT-induced chronic cardiotoxicity.

ATP content of 4 ANT

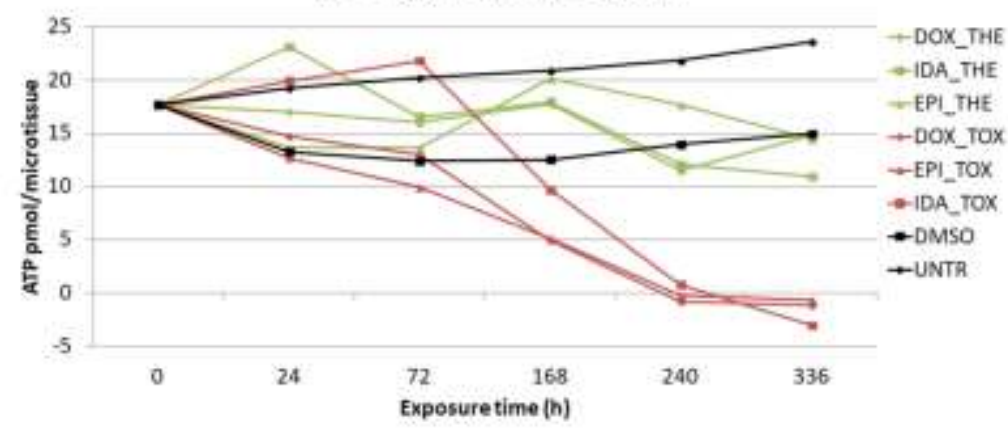

Figure 3: ATP content of ANT-exposed microtissues, indicating a decrease in toxic exposures which is not observed for therapeutic exposures Correction for batch effects between the exposures was done by shifting the curve based on TO set to average of TO for all ANT+controls 


\section{Gene expression changes in chronic cardiotoxicity in vitro}

In order to investigate ANT-induced chronic cardiotoxicity, we analyzed not only differentially expressed genes (DEG; FDR $<0.05$ ) but also changes due to alternative splicing that resulted in differential transcript usage (DTU; FDR $<0.01$ and change $>15 \%)$. Unless otherwise referenced, information regarding structure and functions of genes and proteins were retrieved from the Ensembl ${ }^{[27]}, \mathrm{NCBI}^{[28]}$, Uniprot $^{[29]}$ and GeneCards ${ }^{[30]}$ databases. Figure 4 shows an overview of the sarcomere, with gene expression changes at each time point and whether these genes displayed DTUs. Transcript isoform switch analysis was performed in cases where at least 2 DTUs were identified for a given gene. Figure 5 presents detailed information on sarcomeric DTUs, which includes data from in vitro as well as from human biopsies to simplify comparison later on. Because of the large amount of known transcripts, they were grouped based on overlap between the Uniprot ID they encode. Other groups entailed non-protein-coding transcripts (NPCT), NMD transcripts (transcripts faded to be degraded through nonsense mediated decay) and other variants (proteins of unknown function).

The sarcomere is the contractile unit of muscle cells. The action of sarcomere contraction is facilitated by movement of myosin (thick filaments of the sarcomere) along actin (thin filaments of the sarcomere), a process that is dependent on ATP hydrolysis for necessary conformational changes of myosin in this process. The cardiac muscle $\alpha$-actin (ACTC1) gene displayed increased expression, for DOX exposure only at 168h, but for EPI and IDA exposure at most time points. Furthermore, no DTUs have been observed. This implies an increased amount of protein-coding ACTC1 transcripts, and therefore the ability to produce more actin proteins (proteomics data not available). Since it is known that downregulation of ACTC1 reduces cardiac contractility $^{[31]}$, the increased expression could indicate an increase in contraction, which is one of the symptoms of heart failure. Most genes encoding for myosin subunits are highly affected by ANT exposure, with the 3 ANTs inducing similar changes. While expression of cardiac myosin light chain (MYL) 2 is decreased, 
increased expression is found for MYL3 and MYL4. Next to increased expression, MYL4 also showed DTUs (Figure 5) in which an isoform switch was observed from MYL4-209 (NMD-transcript) to MYL4-202 (encoding P12829, 197aa). This implies an increase in cellular MYL4 protein content. Proteomics measurements (Figure 4C) for EPI and IDA exposure indeed showed an increase in MYL4 protein after $72 \mathrm{~h}$, though this is not observed for DOX. MYL4 is a protein isoform highly expressed in fetal ventricles, which has been found to be re-expressed in various heart diseases. An increase in MYL4 indicates adaptation of the heart, since even small amounts increase shortening velocity and maximum isometric force ${ }^{[32]}$. ANT-induced changes were also observed for the myosin heavy chains (MYHs). Sporadic downregulations of $M Y H 7$ (encoding slow $\beta$ myosin) combined with an upregulation of $M Y H 6$ (encoding fast $\alpha$-myosin) was observed. While no DTUs were detected for $M Y H 7$, the proteomics measurements were not in line with the observed gene expression changes. Instead, after $72 \mathrm{~h}$ of exposure, protein content of MYH7 increased. This contradiction may imply other regulatory mechanisms to be involved. The upregulation of $M Y H 6$ was decreasing in magnitude over time, which was especially noticeable after exposure with DOX and EPI. Gene expression changes after IDA exposure were larger compared to DOX and EPI, and also induced an isoform switch from MYH6-203 (NPCT: retained intron) to

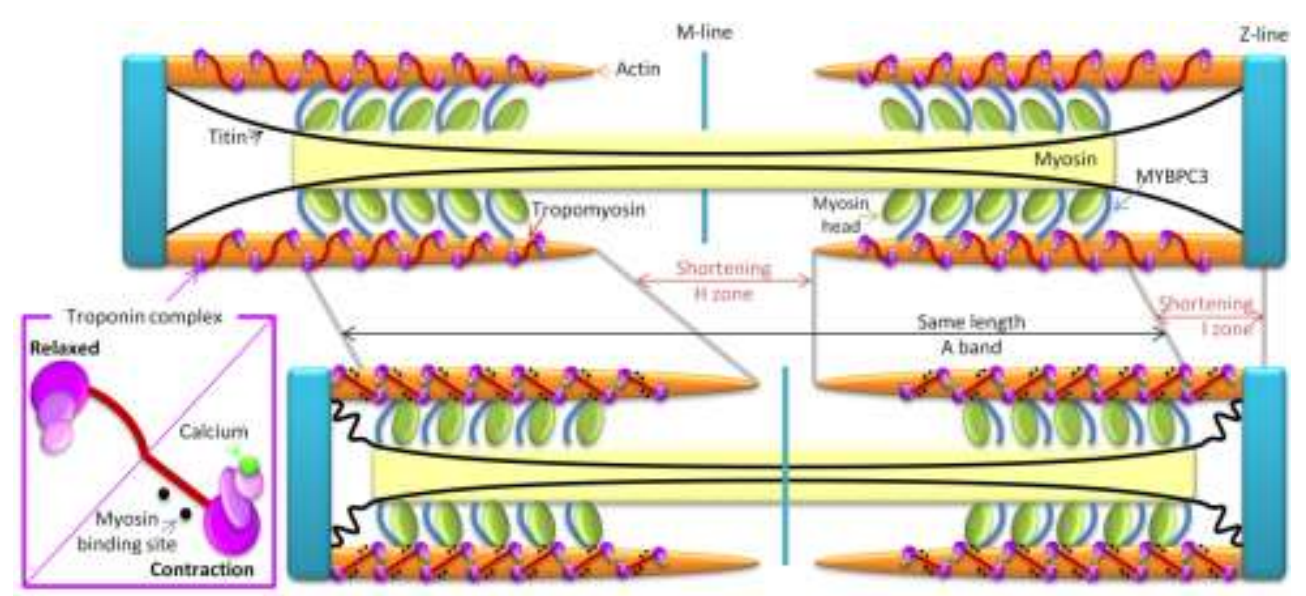

Figure 4A: Schematic representation of the sarcomere, depicting the structure and the localization of involved proteins in order to clarify description of protein functions in the main text 
•. C Chapter 4: Dififerential transcript usage . .
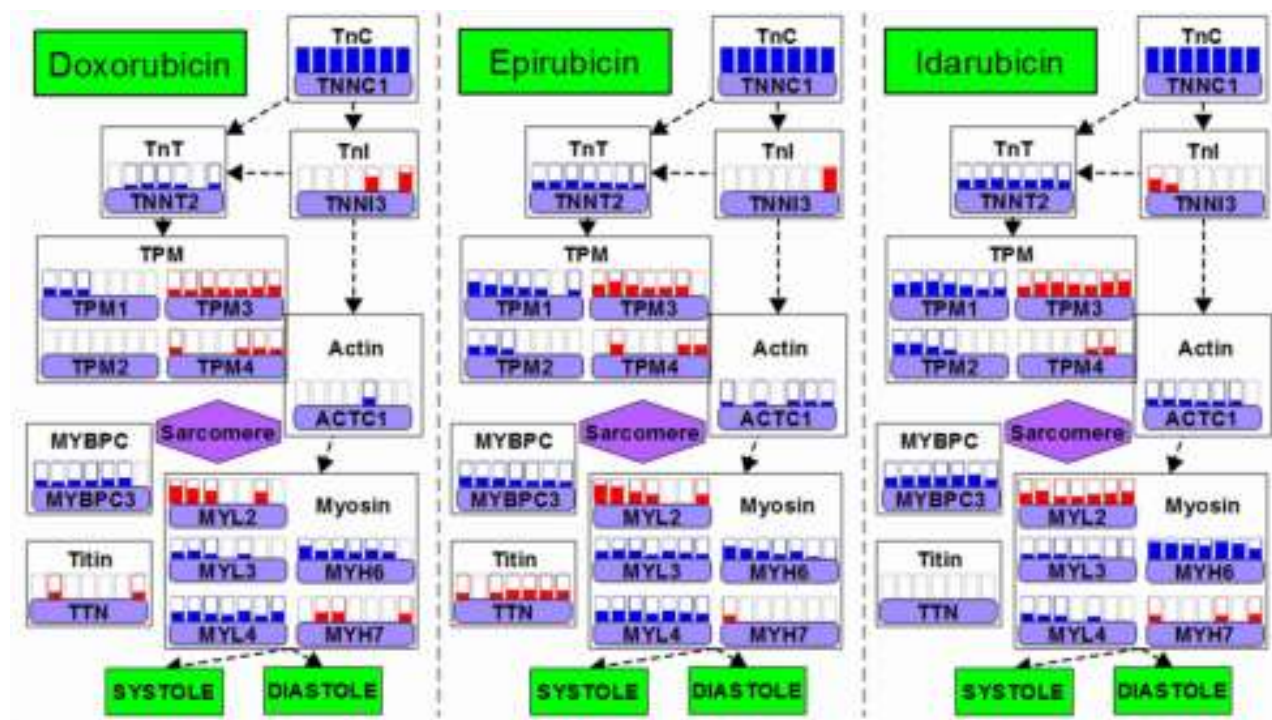

Figure 4B: ANT-induced gene expression changes affecting the sarcomere (blue = upregulation; red $=$ downregulation)

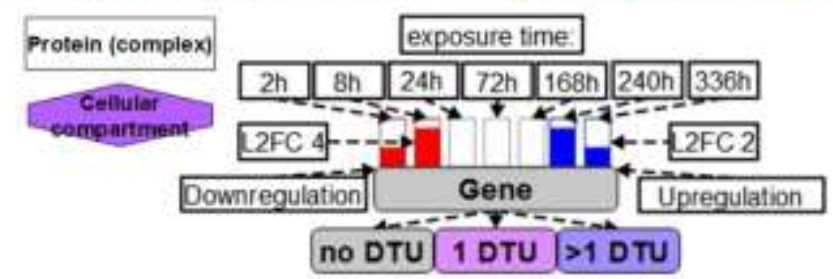

TnC (P63316)
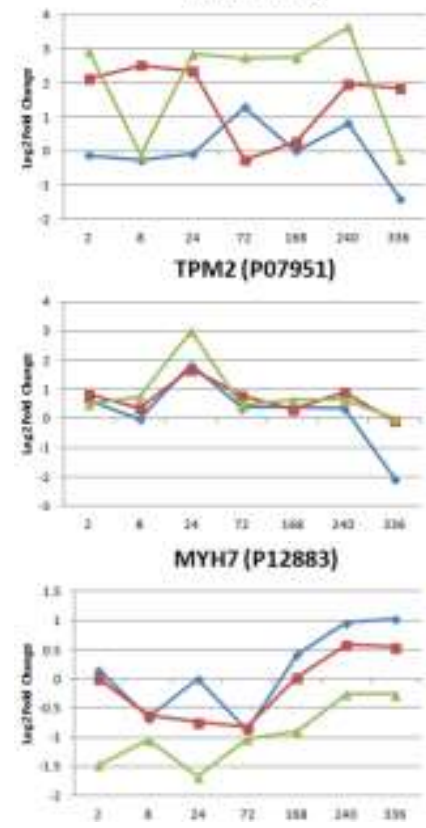

$\operatorname{TnT}$ (P45379)
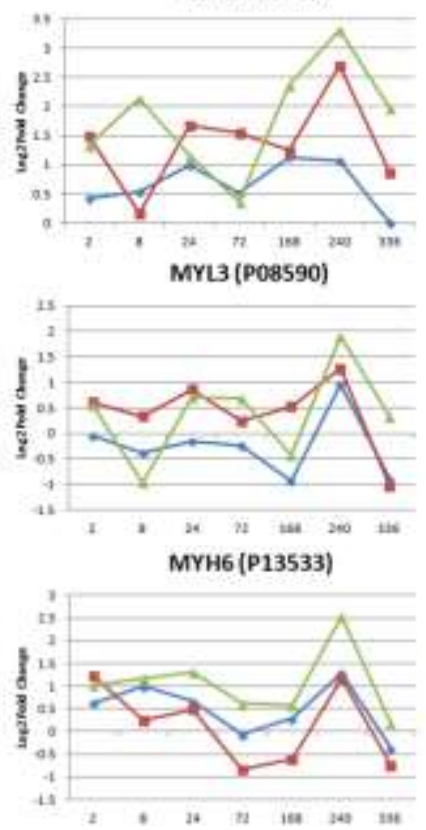

TPM1 (P09493)
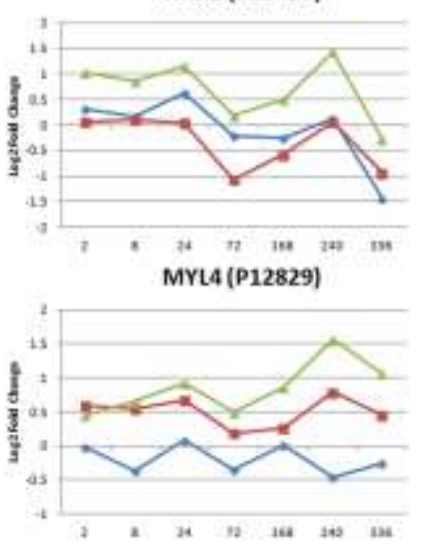

$\rightarrow-\infty O x$

- $=$ EPI Figure 4C: ANT-

$-10 \mathrm{~A}$ induced changes affecting sarcomeric protein content 
MYH6-202 (encoding P13533). These changes in gene expression imply increased protein content that slowly decreased over the course of the exposure to any of the 3 ANT, although demonstrating overall higher levels for IDA exposure. The actual proteomics measurements of MYH6 (Figure 4C) did correlate with this expectation, except for the protein peak observed at $240 \mathrm{~h}$ of exposure. This observed increase of fast $\alpha$-myosin contradicts the reported $\alpha$-to- $\beta$ myosin shift in cardiomyopathy and failing (left or right) ventricle. While the $\alpha$-to- $\beta$ myosin shift is thought to preserve energy, the decreased contractile function is disease-promoting and is therefore classified as a maladaptation response and could lead to toxicity. Therefore, the $\beta$-to- $\alpha$ myosin shift observed by us (and reported in failing myocardium) indicates increased contractile velocity, which may be the cells compensation mechanism in reaction to ANT-induced stresses ${ }^{[31,33,34]}$. Taken together, our observations indicate an increase in contraction rate and/or force in response to ANT exposure due to changes in actin and myosin.

While actin and myosin actually facilitate contraction of the sarcomere, their actions are regulated by tropomyosin (TPM) and troponin (Tn). TPM is wrapped around actin in a helical formation, blocking myosin binding sites, thereby preventing contraction. Four genes encode TPM variants, of which TPM- $\alpha$ (encoded by TPM1) is most abundant in cardiac tissue, followed by TPM- $\beta$ (encoded by TPM2). In a healthy heart, TPM- $\alpha$ and TPM- $\beta$ are present in a ratio of 4.8:1. Changes in this ratio resulting the abundance of the $\beta$-isoform, induces reduced contraction and relaxation rates ${ }^{[31]}$. Upregulation of TPM1 was found for the 3 ANTs, though for DOX this was only in early time points. Furthermore, DTUs were observed during DOX and IDA exposure (Figure 5). Both present an isoform switch from TPM1-223 (encoding HOYKZ6, 70aa) to TPM1207 for DOX and TPM1-220 for IDA (both encoding main protein P09493, 284aa). However, the increase in protein-coding transcripts for all 3ANT was not reflected in the actual proteomics measurements (Figure 4C), which showed a decrease of TPM1 proteins in the exposed cells. One possibility for this discrepancy could be miRNAs that prevent translation of the mRNA into protein. For TPM2, upregulations were detected in early time points during EPI and IDA exposure, while DOX did not show expression 
changes. This compound-specific difference was not observed in the proteomics results, which indicated a mostly stable TPM2 protein content except for a temporary increase at $24 \mathrm{~h}$ for all 3ANT and a decrease at 336h for DOX. Furthermore, TPM3 was downregulated during the exposure of all 3ANTs, as was TPM4 at some time points. An isoform switch was identified for TPM4 during EPI and IDA exposure. A decrease of TPM4-201 (encoding main protein P67936, 248aa) and an increase of TPM4-205 (encoding K7ENT6, 179aa) were observed for both exposures. Additionally, an increased TPM4-206 (NPCT: retained intron) was found during IDA exposure (proteomics data not available). Since the main cardiac tropomyosins ( $\alpha$ and $\beta$, encoded by TPM1 and TPM2) remain largely unchanged at the proteomics level, it is unlikely that tropomyosins are causal for ANT-induced heart failure.

The Tn complex is the main regulatory component of muscle contraction. It consists of 3 proteins: 1) TnC, which regulates muscle contraction in response to calcium binding, 2) Tnl, which contains binding sites for actin, and 3) TnT, which contains binding sites for TPM. In resting state, $\mathrm{TnC}$ is loosely bound to $\mathrm{Tnl}$, which is bound to actin. This results in a protein conformation that positions the TnT so that the bound TPM spirals around the actin to block myosin binding sites. When calcium binds to TnC, its interaction with Tnl strengthens, resulting in detachment form actin. This change in the Tn complex causes TPM to shift and expose myosin binding sites, facilitating contraction of the sarcomere. Interestingly, next to being part of this regulatory process, $\mathrm{TnT}$ and $\mathrm{Tnl}$ detected in circulation are used as biomarkers for the assessment of heart failure in the clinic $^{[35]}$. Gene expression of TNNI3 was mostly unchanged, except for sporadic downregulations (proteomics data not available), while TNNC1 was highly upregulated throughout ANT exposure, which was not reflected by the highly variable proteomics results (Figure 4C). No DTUs were detected for either TNNI3 or TNNTC1, which is in line with literature reporting no protein isoforms changes during development or pathological conditions ${ }^{[31]}$. Like TNNC1, TNNT2 displays stable upregulation during ANT exposure, though with smaller expression changes.

Furthermore, TNNT2 was the only component of the Tn-complex depicting DTUs (Figure 5). During DOX exposure a transcript-isoform switch was observed from 

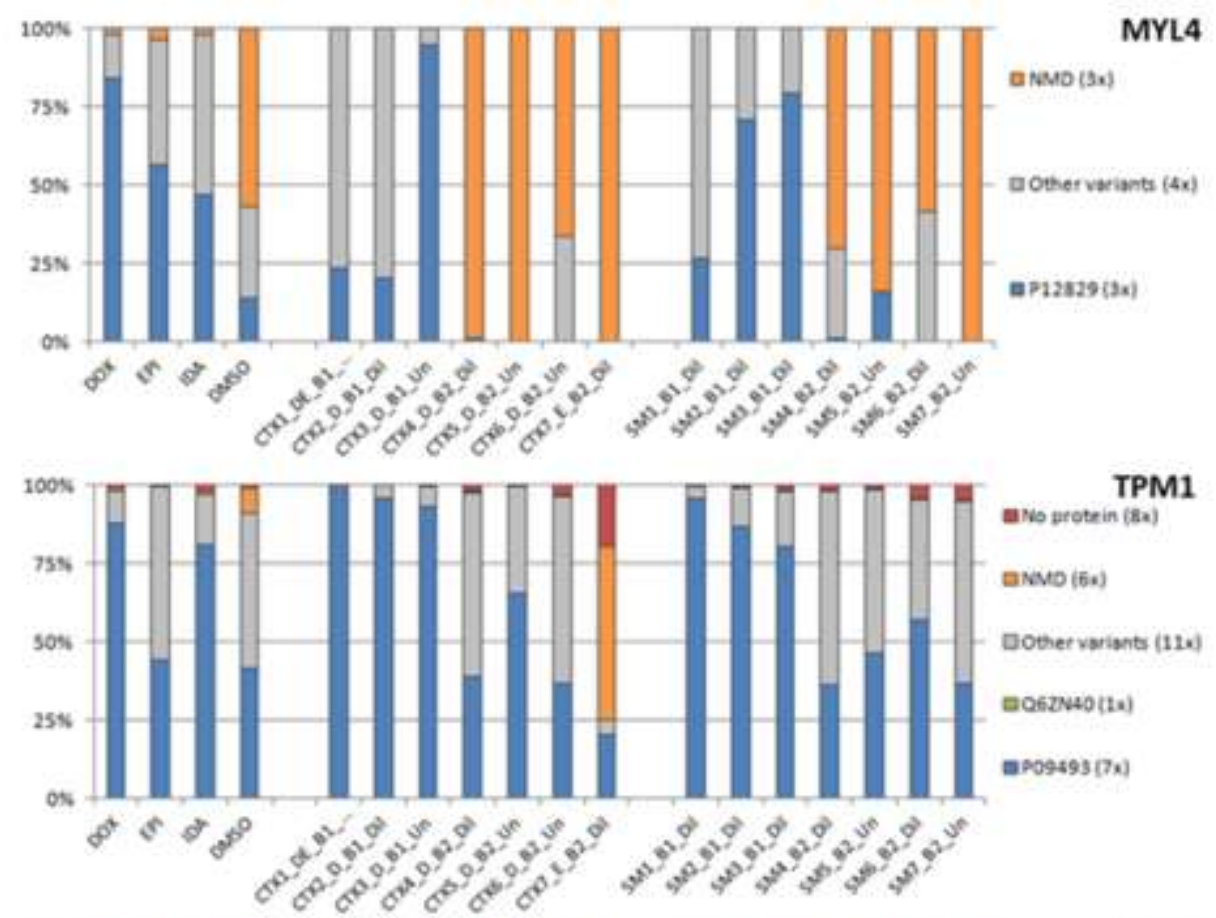

TPM1

ans prote in (Ba)

anvo (6.)

Dober variants (11.)

Dosento(ta)

อค09493 [7N]

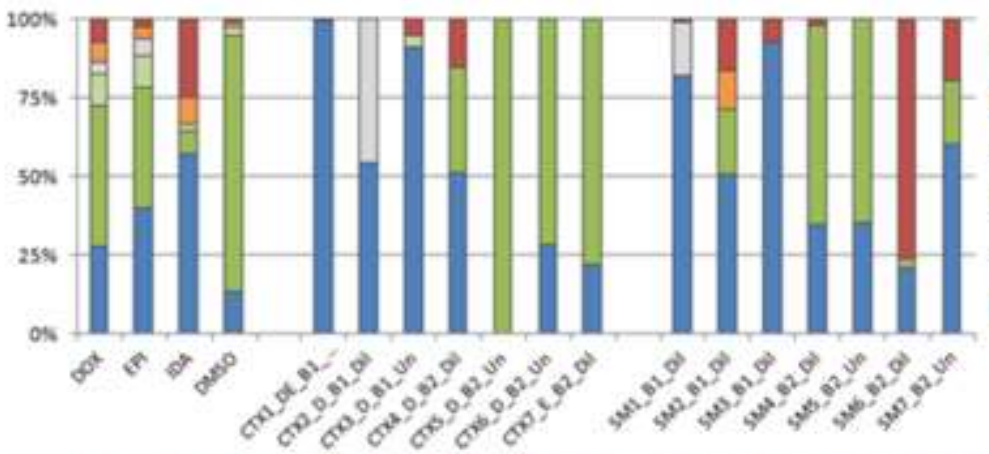

TPM3

a No proteio (0x)

anMD (3.e)

Dothervariants $[\mathrm{Bx}$ )

Dosimes (2s)

a asvers (2.)

apo6753(7x)

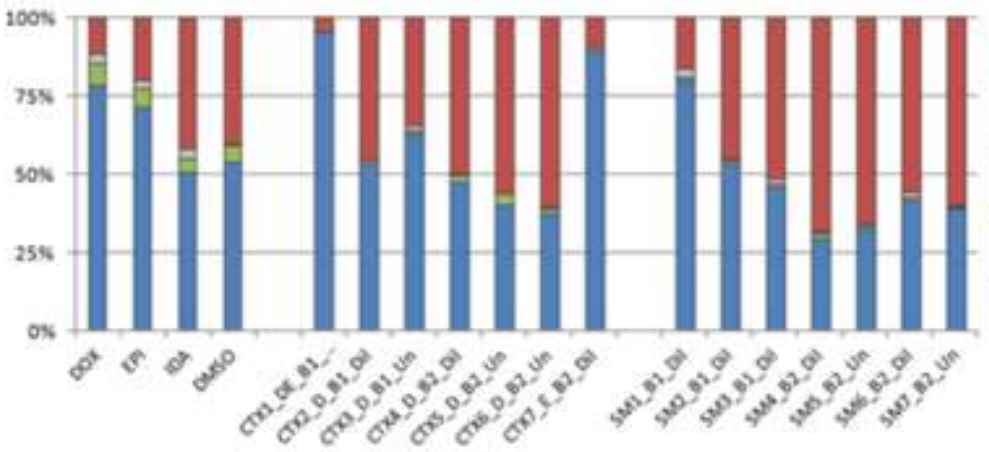

TNNT2

ave protein (11x)

Dother variants $(6 x$ )

Ea725s4 (2x)

ap45379 (6x)

Figure 5: DTU of sarcomeric genes in human biopsies and in vitro micro tissues. Graphs display the percentage of transcript usage for in vitro exposures (bar 1 -4) and in vivo samples (bar 5-18). 

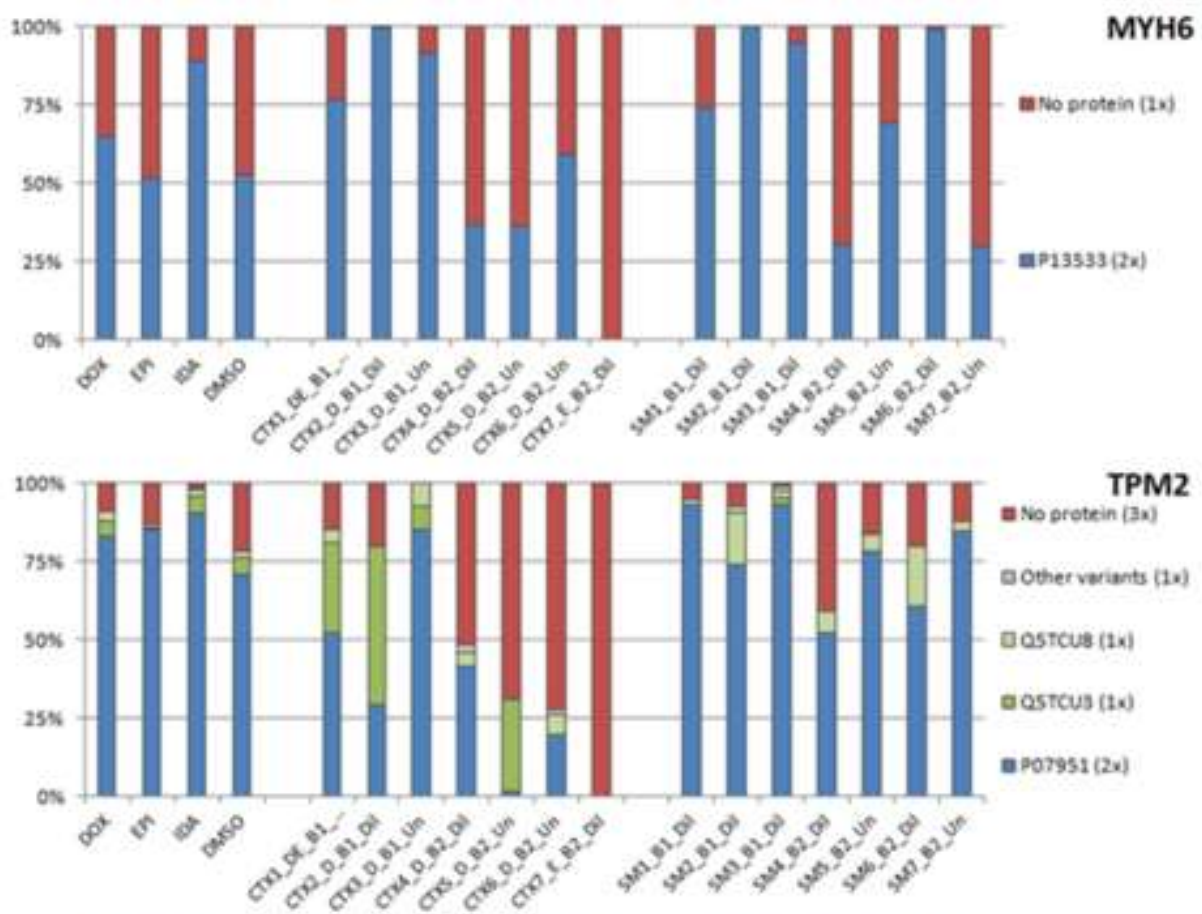

TPM2

No protein (3x)

oother variants (1x)

gostcus (12)

aestous (120)

a por9si (2x)

$100 \times$

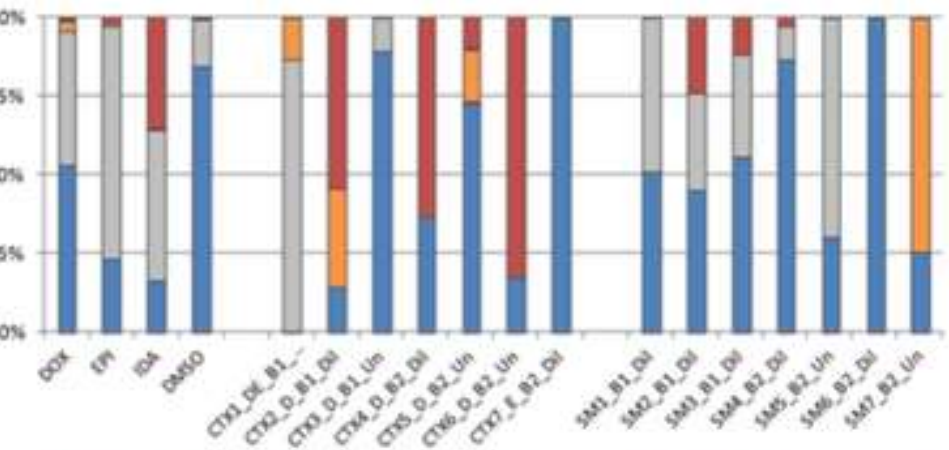

TPM4

No protein (5x)

DNMO (2.)

Dothet variants (3.)]

aP57936 (20)

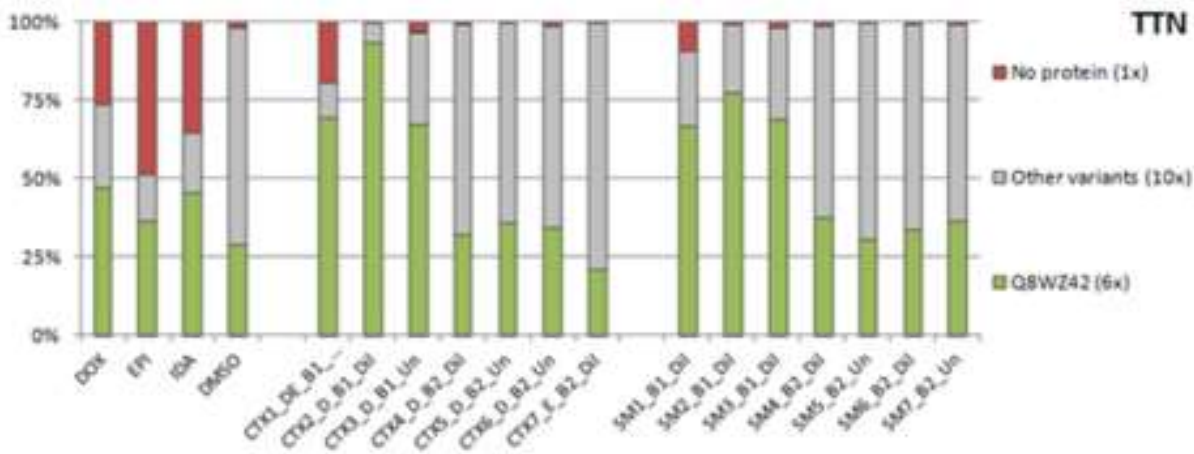

Figure 5 (continued): DTU of sarcomeric genes in human biopsies and in vitro micro tissues. Protein coding transcripts are depicted in cold colors (Main transcript in blue \& other variants in green), warm colors depict transcripts not resulting in proteins (Non-coding transcripts in red \& NMD transcripts in orange) 
TNNT2-217 (NPCT: retained intron) to TNNT2-207 (encoding P45379, 285aa). The decrease of the TNNT2-217 transcript was also observed during EPI exposure, though the counter reaction of this change did not pass our significance threshold. TNNT2-207 encodes the cTnT3 isoform, which is the only protein isoform expressed in healthy adult hearts. In the failing heart, re-expression of fetal cTnT4 (encoded by TNNT2-203) has been reported, which was not observed in our in vitro data ${ }^{[36]}$. The proteomics measurements of $\mathrm{TnT}$ (Figure $4 \mathrm{C}$ ), although showing high variability between compounds and time points, seem to indicate an increasing trend throughout ANT exposure, which is in line with the changes in gene expression. While no aberrant transcript variants were expressed, changes in expression of TnTs may indicate alterations in protein turn-over. After cardiac injury, increased degradation of TnTs has been reported, which consequently results in elevated TnT concentrations in the circulation that are used as biomarkers to detect cardiac injury ${ }^{[37]}$. Finally, changes in expression and protein turnover may affect the ratio in which the Tn subunits are available in the cell. Normally, 1 Tn complex (containing 1 of each subunit) is distributed every 7 actin monomers along the thin filament ${ }^{[37]}$. Changes in this distribution may impact on contraction regulation, calcium sensitivity, ATPase activity and overall cardiac functioning ${ }^{[38]}$.

The two remaining sarcomeric elements, cardiac Myosin-Binding Protein C (MYBPC3) and titin (TTN), are essential for maintaining the structure of the sarcomere. MYBPC3 is found in the crossbridge-bearing zone (C-region) where it binds myosin, actin and titin. Expression of MYBPC3 was stably upregulated after ANT exposure (proteomics data not available). MYBPC3 has only been implicated in heart failure due to mutations, not due to changes in expression or isoform switches ${ }^{[31]}$. Finally, titin connects the Z-line with the $\mathrm{M}$-line forming the base for the assembly of the contractile machinery. It unfolds during relaxation and refolds during contraction. Though a downregulation throughout the exposer was only found during exposure with EPI (proteomics data not available), the DTU of TTN-206 (Figure 5) ranked as the most significant one in all 3ANTs (FDR: $6.96 \cdot 10^{-32}, 2.04 \cdot 10^{-31}$ and $1.38057 \cdot 10^{-39}$ for DOX, EPI and IDA, resp.). This change constitutes a decrease in TTN-206 (H7C1P9, 962aa) combined with an increase 
in TTN-213 (NPCT: retained intron). Compared to the main TTN protein (Q8WZ42, $\approx 30.000$ aa), the H7C1P9 is a smaller variant due to incomplete $5^{\prime}$ and 3 'coding regions in the transcript. Though the function of this truncated variant is not known, the disappearance of this transcript might influence the stiffness of the sarcomere and might impacting the ability to contract.

Overall, our in vitro analysis reveals major effects of anthracyclines on the sarcomere. Mechanistically, most noteworthy are the changes in actin and myosin facilitating the actually contraction of the sarcomere - which imply an increase in contraction rate and/or force in response to ANT exposure. While tropomyosins and troponins - regulating contraction in response to calcium signaling - did show changes in gene expression, these changes were not clearly reflected by the proteomics data, suggesting they are not causally involved in chronic cardiotoxicity. Because troponins are already used as biomarkers of cardiac injury, the identification of increased TNNT2207 protein-coding transcript might be interesting as an additional biomarker. Finally, titin - a structural component of the sarcomere - revealed the highly significant decrease of the TTN-206 transcript. Even though the function of this truncated variant is unknown, this constitutes the most promising target to be used as a biomarker for early detection of ANT-induced chronic heart failure.

\section{Differential transcript usage in human biopsies of heart failure patients}

In order to assess if the in vitro observed ANT-induced changes are relevant targets for detecting heart failure in vivo, we also analyzed DTUs of sarcomeric genes in cardiac biopsies of patients with decreased left ventricular function. The analysis included 7 biopsy sets of ANT-treated (cardiotoxicity - CTX) and non-chemotherapy treated (sample matched - SM), matched on sex, age, BMI and LVEF. Table 1 contains the characteristics of these human biopsies.

The analysis of DTUs resulted in 2 important observations (Figure 5). First, because not all biopsies were obtained and sequenced at the same time, a difference between sample pairs 1-3 and 4-7 is clearly noticeable for MYH6, MYL4, TMP1 and TTN. 
This may constitute a batch effect, although it may also be caused by differences in severity of heart failure, since the first batch was more inflicted based on left ventricular ejection fraction (LVEF) and morphology. Furthermore, transcript expressions are highly similar between CTX and its SM. This may then imply a dependency of transcript expression with one of the parameters the samples are matched on. Therefore, the correlation of transcript expression with specific parameters was investigated. No differences between groups were found based on sex or BMI. When comparing CTX vs SM, only TPM2 showed significant changes in transcript expression (Figure 5). The decrease in transcripts coding for P07951 ( $p=0.005 ;$ TPM2-202/203) in CTX compared to SM was the most significant. While both TPM-202 and TPM-203 transcripts encode for this protein, only the TPM-202 transcript is responsible for the observed decrease. Also the counter reaction of increased Q5TCU3 encoding transcript (TPM2-201) and non-protein-coding transcripts (TPM2205/206/207) were significant $(p=0.049)$. The Q5TCU3 and P07951 proteins are both 284aa in length, although they differ in sequence in the 27 aa at the C-terminus end. The switch from non-protein-coding to protein-coding transcripts observed in CTX biopsies was not observed in vitro. This might therefore be an event occurring later in the disease development or it can be caused by a confounding factor since patients are treated with a mixture of drugs instead of a single ANT.

Comparisons based on actual disease parameters revealed a correlation between decreased TTN-206 with increased left ventricular (LV) mass index $(p=0.002)$ and decreased LVEF ( $p=0.018)$. The biopsies indicated a switch from TTN-206 (encoding H7C1P9, 962aa) to TTN-212 (encoding Q8WZ42, 26.926aa). The decrease in TTN-206 was also observed in vitro, however, the increase in TTN-212 was not. TTN-212 encodes a protein isoform known as N2B. In the adult heart, the ratio of the 2 TTN isoforms N2B and N2BA (encoded by TTN-216) determines the passive myocyte stiffness ${ }^{[39]}$. Increased N2B, also detected in DCM mouse models ${ }^{[40]}$, and a changed N2B:N2BA ratio would lead to stiffer less compliant sarcomeres. While not statistically significant, TTN-206 was the only transcript with a p-value close to significant (0.07) when comparing between dilated and unaffected morphologies. Taken together, these 
correlations clearly indicate the involvement of the TTN-206 transcript in heart failure. The increase in TTN-213 (NPCT: retained intron) observed in vitro, was not observed in vivo, though statistical comparisons revealed a relation between increased TTN-213 and more severe New York Heart Association (NYHA) classification (class 2 "Slight limitation of physical activity" vs class 3 "Marked limitation of physical activity", p=0.02). Similar to TTN-206, decreased TPM1-223 (encoding HOYKZ6, 70aa) was correlated with increased LV mass index $(p=0.007)$ and decreased LVEF ( $p=0.029)$. Biopsies revealed a switch from TPM1-223 (encoding HOYKZ6, 70aa) to transcripts encoding the main protein (P09493, 284aa; TPM1-201/202/204/206/207/219/220), with TPM1-220 contributing the most to the observed change. The same switch was observed in vitro, thereby confirming its relevance for ANT-induced chronic cardiotoxicity. A switch between MYL4-205/206/209 (NMD-transcripts, $p=0.035$ ) to MYL4-203 (encoding A0A1C7CYY4, 196aa) and MYL4-201/202/208 (encoding main protein $\mathrm{P} 12829,197 \mathrm{aa})$ has only been found to correlate with decreased LVEF ( $p=$ 0.03). This switch from NMD-transcripts to main protein transcripts was also observed in our in vitro analysis, implicating its relevance for heart failure. Interestingly, the MYL4-210 transcript (encoding I3L1K6, 172aa) could discriminate between CTX and SM patients within NYHA class3 $(p=0.02)$ and LVEF class $1(p=0.044)$, with decreased expression for CTX patients. Whether this is a biological effect or caused by confounding factors cannot be determined. Furthermore, a decrease of TPM3-207 (encoding Q5VU61, 223aa) was correlated with LVEF ( $p=0.029)$. The counter reaction, a decrease in P06753 encoding transcripts ( $\approx 250$ aa, TPM3202/204/205/206/210/211/212), with TPM3-206 and TPM3-210 contributing most to the observed change, correlated with NYHA severity $(p=0.049)$ and fractional shortening ( $\mathrm{p}=0.047)$. In vitro, these transcripts were not discussed because only a single DTU was observed. However, this entailed the decrease of TPM3-207, similar to in vivo. Finally, correlated with increased LV mass index was an increase of TNNT2 protein-coding transcripts $(\mathrm{p}=0.0004, \mathrm{P} 45379, \approx 280 \mathrm{aa}$, TNNT2201/202/205/206/207/224) and a decrease in non-protein-coding transcripts ( $p=0.0005$, TNNT2-212/215/216/217/218/219/220/221/222/223/225). Though all the increased TNNT2 transcripts encode proteins with the same UniProt ID, the transcripts 
differ in length and function due to alternative exon usage. Co-expression of distinct cardiac TnT transcripts reduced cardiac performance in mice due to decreased contractility and relaxation velocity ${ }^{[31]}$. The observed changes are mostly due to an increase in TNNT2-207 (encoding P45379, 285aa) with a decrease of TNNT2-217(NPCT: retained intron), the exact same switch as was observed in vitro, the biological result may therefore just be an increase in the normally expressed TNNT2-207 transcript.

In summary, despite the heterogeneity of the human biopsies, the interesting DTU observed in vitro (TTN-206 and TNNT2-207) were also observed in the human biopsies, making them clinically relevant. First, the decrease of the TTN-206 transcript was the most significantly finding in vitro (FDR: $6.96 \cdot 10^{-32}, 2.04 \cdot 10^{-31} \& 1.38057 \cdot 10^{-39}$ for DOX, EPI \& IDA, resp.) and was correlated in vivo with increased left ventricular (LV) mass index $(p=0.002)$, decreased LVEF $(p=0.018)$ and was the only transcript close to significant $(P=0.07)$ when comparing dilated vs undilated morphologies. The switch form TTN-206 (encoding H7C1P9, 962aa) to TTN-212 (encoding Q8WZ42, 26.926aa) changes the expression of a truncated titin protein variant. These truncated variants have the ability to impact the structural organization of sarcomeres and to disturb regulation of sarcomeric passive force generation and reduce the efficiency of contraction ${ }^{[26,41,42]}$. Second, troponin-T is already used as a tissue specific biomarker to detect anthracycline-induced cardiotoxicity, although in this case it concerns troponinT proteins that have been introduced into the bloodstream due to myocardial damage ${ }^{[2,43]}$. Next to being a leakage biomarker, ANT exposure induces upregulation of TNNT2 gene expression combined with a transcript-isoform switch from nonprotein-coding transcripts to the TNNT2-207 transcript which encodes the most abundant protein isoform (cTnT3) in human heart. This DTU correlated with increased LV mass index $(p<0.0005)$ in vivo. As stated before, changes in distribution of Tn subunits along the thin filament can impact on contraction regulation, calcium sensitivity, ATPase activity and overall cardiac functioning ${ }^{[38]}$. Overall, the increased TNNT2-207 transcript usage may facilitate identifying susceptibility to heart failure before myocardial damage occurs. 


\section{Conclusion}

Overall, the current study shows the added value of biological information to be gained from DTU analysis as compared to standard gene expression analysis. This resulted in the discovery of 2 potential new biomarkers (TNNT2-207 and TTN-206) for early detection of ANT-induced cardiotoxicity and heart failure in general. Especially the observed decrease in TTN-206 is highly promising as a biomarker because it correlated with increased LV mass index, decreased LVEF and can possibly distinguish between dilated and unaffected morphologies.

\section{Closing remarks}

The results presented in this analysis need to be put in the context of the cell model consisting of induced pluripotent stem cells (iPSC)-derived cardiomyocytes (CM). iPSCs can be derived from any type of mature cells through the introduction of key transcription factors ${ }^{[44]}$. Similar to human pluripotent stem cells (hPSCs) derived from embryos, iPSCs can be differentiated into cells of any other cell type, which is of great benefit to research and medical technology. Furthermore, because iPSCs do not require destruction of embryos, they have the additional benefit of not dealing with ethical implications. However, both are subjected to genomic instability, which was first reported in early 2000s for hPSC ${ }^{[45-47]}$ and in 2010 for iPSCs ${ }^{[48]}$. Both PSCs display similar genomic changes, ranging from chromosome aneuploidies to gene duplications, deletions or point mutations. Furthermore, the type and frequency of the abnormalities are not related to the applied reprogramming methods ${ }^{[49]}$. Until now, 3 sources for genetic variations have been identified. First, any variations pre-existing in parental somatic cells are amplified because each iPSC colony results from a single somatic cell. Second, during differentiation, changes in culture conditions induce new selective pressures, favoring genetic variants that are best adapted. Finally, prolonged culturing of iPSC-derived cells has been also shown to induce additional mutations. Though more comprehensive studies are needed to obtain reliable frequencies of genetic abnormalities in PSC cells, current reports range from 10 protein-coding mutations to hundreds of point mutations (for review see ${ }^{[44,49]}$ ). 
Due to this multitude of sources for genetic variation, no 2 batches of iPSC-derived cells are identical, giving raise to batch effects in the current data. This could bias some results because DTUs observed in vitro may also be due to different single nucleotide polymorphisms (SNPs) between the batches, which we cannot correct for in our current dataset.

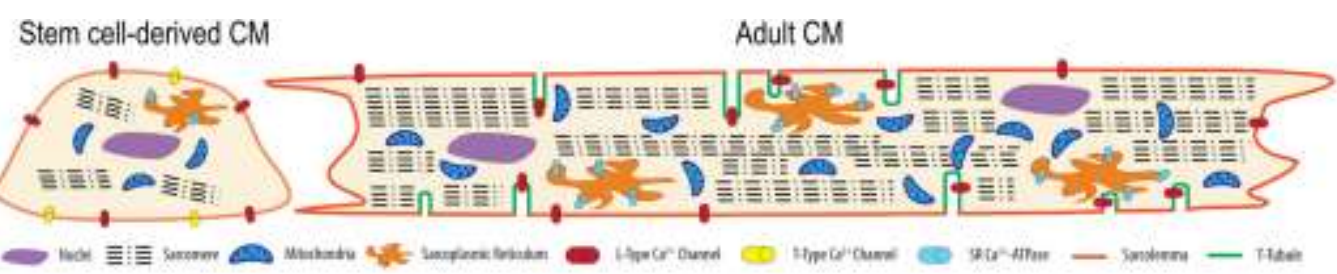

Figure 6: Structural differences between stem cell-induced $\mathrm{CM}$ and adult $\mathrm{CM}$ (adapted from ${ }^{[50]}$ ). Compared to adult $\mathrm{CM}$, stem cell-derived CM contained less structured sarcomeres and had a decreased sarcomeric area and volume

Another drawback of PSCs-derived cells is that, currently, differentiation does not reach an adult-like phenotype. Rather, the cells retain a fetal-like structure ${ }^{[51,52]}$. Compared to adult CM, stem cell-derived CM contained less structured sarcomeres and had a decreased sarcomeric area and volume (Figure 6) ${ }^{[50]}$. Still, iPSC-derived CMs were able to produce a force which was measurable using video microscopy, which is vital for CM function. Also, they possess functional calcium stores (which is a hallmark of mature $\mathrm{CMs}$ ) and are able maintain $\mathrm{Ca}^{2+}$-signaling ${ }^{[53]}$. Because the sarcomeres of iPSC-derived CMs are functional, drug induced differences can still be detected. However, extrapolation of our findings to adult heart has to be done with some caution. In order to deal with the immaturity of $\mathrm{CMs}$ and possible batch in our data, we regarded possible interesting biomarkers for early detection of anthracycline-induced cardiotoxicity as findings detected both in vitro and in vivo, which nominated myosin light chain 4, tropomyosin 1, troponin-T and titin. Of these, titin and troponin-T turned out to be especially interesting as potential biomarkers (as described above). 


\section{Supplementary materials}

Supplementary figure 1: Heart failure model.

Average gene expression changes (7 time points) for A) therapeutic ANT and B) toxic ANT exposure. Significant changes in gene expression (FDR $<0.05)$ at every time point for C) Therapeutic DOX, D) Toxic DOX, E) Therapeutic EPI, F) Toxic EPI, G) Therapeutic IDA, H) Toxic IDA.

ftp://web.tgx.unimaas.nl/mverheijen/Chapter4/Supplementary_figure1.docx

Supplementary table 1: Significantly overrepresented pathways using 3704 overlapping genes

ftp://web.tgx.unimaas.nl/mverheijen/Chapter4/Supplementary_table1.xlsx 


\section{References}

1 Bernstein, D. Anthracycline Cardiotoxicity Worrisome Enough to Have You Quaking? Circ Res 122, 188190, doi:10.1161/Circresaha.117.312395 (2018).

2 McGowan, J. V. et al. Anthracycline Chemotherapy and Cardiotoxicity. Cardiovasc Drug Ther 31, 63-75, doi:10.1007/s10557-016-6711-0 (2017).

3 Menna, P. \& Salvatorelli, E. Primary Prevention Strategies for Anthracycline Cardiotoxicity: A Brief Overview. Chemotherapy 62, 159-168, doi:10.1159/000455823 (2017).

4 Weintraub, R. G., Semsarian, C. \& Macdonald, P. Dilated cardiomyopathy. The Lancet (2017).

5 Mladěnka, P. et al. Comprehensive review of cardiovascular toxicity of drugs and related agents. Med Res Rev (2018).

6 Cardinale, D. et al. Anthracycline-Induced Cardiomyopathy Clinical Relevance and Response to Pharmacologic Therapy. J Am Coll Cardiol 55, 213-220, doi:10.1016/j.jacc.2009.03.095 (2010).

7 Cardinale, D. et al. Prevention of high-dose chemotherapy-induced cardiotoxicity in high-risk patients by angiotensin-converting enzyme inhibition. Circulation 114, 2474-2481, doi:10.1161/CIRCULATIONAHA.106.635144 (2006).

8 Cappetta, D. et al. SIRT1 activation attenuates diastolic dysfunction by reducing cardiac fibrosis in a model of anthracycline cardiomyopathy. Int J Cardiol 205, 99-110, doi:10.1016/j.ijcard.2015.12.008 (2016).

9 Raj, S., Franco, V. I. \& Lipshultz, S. E. Anthracycline-induced cardiotoxicity: a review of pathophysiology, diagnosis, and treatment. Curr Treat Options Cardiovasc Med 16, 315, doi:10.1007/s11936-014-0315-4 (2014).

10 Verheijen, M. et al. Bringing in vitro analysis closer to in vivo: Studying doxorubicin toxicity and associated mechanisms in 3D human microtissues with PBPK-based dose modelling. Toxicol Lett (2018).

11 Kuepfer, L. et al. A model-based assay design to reproduce in vivo patterns of acute drug-induced toxicity. Arch Toxicol, 1-3 (2017).

12 Richard, $\mathrm{H}$. et al. Prediction of alternative isoforms from exon expression levels in RNA-Seq experiments. Nucleic Acids Res 38, doi:ARTN e112 10.1093/nar/gkq041 (2010).

13 Wang, E. T. et al. Alternative isoform regulation in human tissue transcriptomes. Nature 456, 470-476, doi:10.1038/nature07509 (2008).

14 Trapnell, C. et al. Transcript assembly and quantification by RNA-Seq reveals unannotated transcripts and isoform switching during cell differentiation. Nat Biotechnol 28, 511-U174, doi:10.1038/nbt.1621 (2010).

15 de Souza, T. M. Bits and bytes in toxicogenomics: In silico approaches in liver-based mechanistic toxicology; Chapter 3. (2017).

$16 \mathrm{Xu}, \mathrm{C}$. et al. Simultaneous isolation of DNA and RNA from the same cell population obtained by laser capture microdissection for genome and transcriptome profiling. J Mol Diagn 10, 129-134, doi:10.2353/jmoldx.2008.070131 (2008).

17 Kuepfer, L. et al. in Intracellular Delivery III: Market Entry Barriers of Nanomedicines (eds Aleš Prokop \& Volkmar Weissig) 363-374 (Springer International Publishing, 2016).

18 Bolger, A. M., Lohse, M. \& Usadel, B. Trimmomatic: a flexible trimmer for Illumina sequence data. Bioinformatics 30, 2114-2120, doi:10.1093/bioinformatics/btu170 (2014).

19 Andrews, S. FastQC: a quality control tool for high throughput sequence data. http://www.bioinformatics.babraham.ac.uk/projects/fastqc (2010).

20 Li, B. \& Dewey, C. N. RSEM: accurate transcript quantification from RNA-Seq data with or without a reference genome. Bmc Bioinformatics 12, doi:Artn 323 10.1186/1471-2105-12-323 (2011).

21 Langmead, B. \& Salzberg, S. L. Fast gapped-read alignment with Bowtie 2. Nat Methods 9, 357-U354, doi:10.1038/Nmeth.1923 (2012).

22 Love, M. I., Huber, W. \& Anders, S. Moderated estimation of fold change and dispersion for RNA-seq data with DESeq2. Genome Biol 15, doi:ARTN 550 10.1186/s13059-014-0550-8 (2014).

23 Oliveros, J. C. Venny. An interactive tool for comparing lists with Venn's diagrams. ((2007-2015)).

24 Kamburov, A. et al. ConsensusPathDB: toward a more complete picture of cell biology. Nucleic Acids Res 39, D712-D717, doi:10.1093/nar/gkq1156 (2011).

25 Du, J. L. et al. KEGG-PATH: Kyoto encyclopedia of genes and genomes-based pathway analysis using a path analysis model. Mol Biosyst 10, 2441-2447, doi:10.1039/c4mb00287c (2014).

26 Garfinkel, A. C., Seidman, J. G. \& Seidman, C. E. Genetic Pathogenesis of Hypertrophic and Dilated Cardiomyopathy. Heart Fail Clin 14, 139-146, doi:10.1016/j.hfc.2017.12.004 (2018). 
27 EMBL-EBI. e!Ensembl, <https://www.ensembl.org/>

28 Information, N. C. f. B. NCBI Nucleotide Database, <https://www.ncbi.nlm.nih.gov/nuccore/>

29 Consortium, U. UniProt: Universal Protein Resource, <http://www.uniprot.org/uniprot/>

30 Science, W. I. o. GeneCards ${ }^{\circledR}$ : The Human Gene Database <http://www.genecards.org/>

31 Yin, Z., Ren, J. \& Guo, W. Sarcomeric protein isoform transitions in cardiac muscle: a journey to heart failure. Biochim Biophys Acta 1852, 47-52, doi:10.1016/j.bbadis.2014.11.003 (2015).

32 Yamashita, H. et al. Myosin light chain isoforms modify force-generating ability of cardiac myosin by changing the kinetics of actin-myosin interaction. Cardiovasc Res 60, 580-588, doi:10.1016/j.cardiores.2003.09.011 (2003).

33 Dzau, V. J. \& Liew, C.-C. Cardiovascular genetics and genomics for the cardiologist. (Blackwell, 2007).

34 Krenz, M. \& Robbins, J. Impact of beta-myosin heavy chain expression on cardiac function during stress. J Am Coll Cardiol 44, 2390-2397, doi:10.1016/j.jacc.2004.09.044 (2004).

35 Masson, S., Latini, R. \& Anand, I. S. An update on cardiac troponins as circulating biomarkers in heart failure. Curr Heart Fail Rep 7, 15-21, doi:10.1007/s11897-010-0001-0 (2010).

36 Gomes, A. V., Venkatraman, G. \& Potter, J. D. The miscommunicative cardiac cell - When good proteins go bad. Ann Ny Acad Sci 1047, 30-37, doi:10.1196/annals.1341.003 (2005).

37 Parmacek, M. S. \& Solaro, R. J. Biology of the troponin complex in cardiac myocytes. Progress in Cardiovascular Diseases 47, 159-176, doi:10.1016/j.pcad.2004.07.003 (2004).

38 Wei, B. \& Jin, J. P. TNNT1, TNNT2, and TNNT3: Isoform genes, regulation, and structure-function relationships. Gene 582, 1-13, doi:10.1016/j.gene.2016.01.006 (2016).

39 Gigli, M. et al. A Review of the Giant Protein Titin in Clinical Molecular Diagnostics of Cardiomyopathies. Front Cardiovasc Med 3, 21, doi:10.3389/fcvm.2016.00021 (2016).

$40 \mathrm{Wu}, \mathrm{Y}$. M. et al. Changes in titin isoform expression in pacing-induced cardiac failure give rise to increased passive muscle stiffness. Biophys J 84, 244a-244a (2003).

41 Herman, D. S. et al. Truncations of Titin Causing Dilated Cardiomyopathy. New Engl J Med 366, 619-628, doi:DOI 10.1056/NEJMoa1110186 (2012).

42 Fatkin, D. \& Huttner, I. G. Titin-truncating mutations in dilated cardiomyopathy: the long and short of it. Curr Opin Cardiol 32, 232-238, doi:10.1097/Hco.0000000000000382 (2017).

43 Kitayama, H. et al. High-sensitive troponin T assay can predict anthracycline- and trastuzumab-induced cardiotoxicity in breast cancer patients. Breast Cancer-Tokyo 24, 774-782, doi:10.1007/s12282-0170778-8 (2017).

44 Yoshihara, M., Hayashizaki, Y. \& Murakawa, Y. Genomic Instability of iPSCs: Challenges Towards Their Clinical Applications. Stem Cell Rev Rep 13, 7-16, doi:10.1007/s12015-016-9680-6 (2017).

45 Cowan, C. A. et al. Derivation of embryonic stem-cell lines from human blastocysts. New Engl J Med 350, 1353-1356, doi:DOI 10.1056/NEJMsr040330 (2004).

46 Draper, J. S. et al. Recurrent gain of chromosomes $17 q$ and 12 in cultured human embryonic stem cells. Nat Biotechnol 22, 53-54, doi:10.1038/nbt922 (2004).

47 Enver, T. et al. Cellular differentiation hierarchies in normal and culture-adapted human embryonic stem cells. Hum Mol Genet 14, 3129-3140, doi:10.1093/hmg/ddi345 (2005).

48 Mayshar, Y. et al. Identification and Classification of Chromosomal Aberrations in Human Induced Pluripotent Stem Cells. Cell Stem Cell 7, 521-531, doi:10.1016/j.stem.2010.07.017 (2010).

49 Peterson, S. E. \& Loring, J. F. Genomic Instability in Pluripotent Stem Cells: Implications for Clinical Applications. Journal of Biological Chemistry 289, 4578-4584, doi:10.1074/jbc.R113.516419 (2014).

50 Robertson, C., Tran, D. D. \& George, S. C. Concise Review: Maturation Phases of Human Pluripotent Stem Cell-Derived Cardiomyocytes. Stem Cells 31, 829-837, doi:10.1002/stem.1331 (2013).

51 Nunes, S. S., Miklas, J. W. \& Radisic, M. Maturation of stem cell-derived human heart tissue by mimicking fetal heart rate. Future Cardiol 9, 751-754, doi:10.2217/fca.13.71 (2013).

52 Batalov, I. \& Feinberg, A. W. Differentiation of Cardiomyocytes from Human Pluripotent Stem Cells Using Monolayer Culture. Biomark Insights 10, 71-76 (2015).

53 Bedada, F. B., Wheelwright, M. \& Metzger, J. M. Maturation status of sarcomere structure and function in human iPSC-derived cardiac myocytes. Bba-Mol Cell Res 1863, 1829-1838, doi:10.1016/j.bbamcr.2015.11.005 (2016). 

Chapter 5

\title{
Assessment of microRNA \& circular
}

\section{RNA interactions is crucial for}

\section{understanding post-transcriptional}

\section{mechanisms}

\author{
Marcha C.T. Verheijen ${ }^{1}$ \\ Y. Schrooders ${ }^{1}$ \\ O. Clayton ${ }^{2}$ \\ R. Nudischer ${ }^{2}$ \\ N. Selevsek ${ }^{3}$ \\ R. Schlapbach ${ }^{3}$ \\ H. Gmuender ${ }^{4}$ \\ S. Gotta \\ R. Gupta ${ }^{1}$ \\ J. Ochoteco ${ }^{1}$ \\ J. Kleinjans ${ }^{1}$ \\ F. Caiment $^{1}$
}

In preparation 


\section{Abstract}

According to the central dogma of biology, information flows from DNA to RNA to protein, with proteins being responsible for an observed phenotype. This model results in the transcriptome being used as a proxy for the proteome. As a consequence, current transcriptomics analysis disregards the influence post-translational processes which influence protein synthesis and its resulting phenotype. Overall, we hypothesize that identification of toxicant-induced changes in post-transcriptional mechanisms will aid toxicological assessments which use transcriptomics analysis. In the current pilot study, we use an in vitro human cardiac cell model exposed to doxorubicin (DOX), a chemotherapy agent known to cause cardiotoxicity, as a case study for the genomewide assessment of post-transcriptional miRNA-circRNA regulation of translation. We have designed a scoring system for the assessment of interactions between mRNA, miRNA and circRNA, based on their expression values and predicted interactions. After optimization and validation of our scoring system through correlation analysis with the proteome, we identified a DOX-induced increase of translational inhibition, which may play a role in DOX-induced cardiotoxicity. Furthermore, the scoring system enabled us to estimate the amount of translatable mRNAs. The fact that these estimated translatable mRNAs resulted in biologically relevant improvements of correlation with the proteome indicated that assessment of post-transcriptional mechanisms is indeed of added value for assessing toxicological processes when analysing transcriptomics data. 


\section{Introduction}

The well-known central dogma of biology states that information flows from DNA to RNA to protein, with proteins being the functional component resulting in a phenotype ${ }^{[1-3]}$. Using this model, changes in RNA levels are assumed to induce variation in protein abundances which lead to a different phenotype ${ }^{[3,4]}$. This assumption is the basis of most gene-expression analysis in which significant gene expression changes between normal and experimental conditions are investigated. The resulting up- and down-regulated genes are used as evidence for observed changes in phenotype ${ }^{[3,5]}$. A downside of this assumption is current genome-wide expression analysis disregards the influence these post-translational modification mechanisms ${ }^{[4,10]}$. The dependence on RNA expression analysis for research is partly due to the relative ease to obtain genome-wide coverage in contrast to proteins ${ }^{[6]}$. While protein measurements using mass spectrometry provide quantitative data, due to difficulty in detecting low-abundance proteins it is not suited for whole-proteome analysis and typically several hundreds to a few thousand of high abundance proteins are analyzed that only cover a small portion of the genome ${ }^{[4,7]}$. While it is reassuring to the scientific community that differentially expressed RNAs and their protein products correlate significantly better compared to non-differentially expressed transcripts ${ }^{[6]}$, most often notoriously poor correlations between the transcriptome and the proteome are reported ${ }^{[1,2,10]}$. A substantial degree of this discrepancy may be explained by post-transcriptional processes which strongly impact on the ultimate protein synthesis ${ }^{[2,6]}$. These include, but are not limited to, mRNA modifications ${ }^{[16]}$, alternative splicing ${ }^{[17]}$, microRNA mediated regulation ${ }^{[18]}$, long non-coding RNA regulation $^{[19]}$, translational efficiency ${ }^{[20]}$ and RNA decay ${ }^{[21]}$. While not all of these mechanisms can be analyzed on a genome wide scale, sequencing protocols enable analyzing several of these events.

Overall, we hypothesize that identification of toxicant-induced changes in posttranscriptional mechanisms will aid toxicological assessments which use transcriptomics analysis. As a first step to address this hypothesis, we conducted a 
pilot study in which we use doxorubicin (DOX)-induced cardiotoxicity in vitro as case study for assessing post-transcriptional miRNA-circRNA regulation on a genome-wide scale through the use of a scoring system of our own design.

DOX is a chemotherapeutic agent which is known to cause dose-dependent cardiotoxic side-effects, even at therapeutic dose, because cytotoxicity contributes to its efficacy. The onset of DOX-induced cardiotoxicity is difficult to predict, because it can manifest as a chronic cardiotoxic phenotype years after the treatment has ended and because the involved mechanisms are only partially understood ${ }^{[29-33]}$.

MicroRNAs (miRNAs) are small non-coding RNAs of approximately 22 nucleotides ${ }^{[18,22]}$. They inhibit mRNA translation by binding to the $3^{\prime}$ untranslated region $^{[23]}$. This binding depends on complementary base pairing of 2-8 nucleotides in the 5 'end of the miRNA, which is known as the seed region. Because this binding region is short, a single miRNA can interact with hundreds of target transcripts and a single transcript can be targeted by multiple miRNAs ${ }^{[24,25]}$. Next to base pairing, translational inhibition is also dependent on the expression levels of both the miRNA and its transcript target ${ }^{[24]}$. Additionally, while miRNAs are regulators of mRNA translation, they are themselves regulated by another class of non-coding RNAs, the circular RNAs (circRNAs). CircRNAs are closed continuous loops without 5'caps or 3'tails which are generated through head-to-tail mRNA splicing. They are evolutionary conserved non-coding RNAs that are more stable than their linear counterparts due to degradation-resistance ${ }^{[26,27]}$. CircRNAs containing miRNA seed regions are known as miRNA sponges. Due to an increased stability and greater miRNA binding capacities compared to linear transcripts ${ }^{[26]}$, circRNAs can sequester miRNAs away from linear transcript targets, thereby preventing inhibitory effects of the miRNAs on translation $^{[28]}$.

To assess whether DOX is able to induce changes in miRNA-circRNA regulation of translation, we used a dataset obtained from DOX-exposed human cardiac microtissues, consisting of ribo-depleted RNA sequencing data, miRNA sequencing data and proteomics data. For assessment of genome-wide interactions between 
mRNA, miRNA and circRNA, we designed a scoring system based on mRNA, miRNA and circRNA expression values and predicted interactions.

In this article, we first give a demonstration of our scoring system by focussing on one transcript as an example. Thereafter, we optimized and validated the interpretation of the obtained scores through correlation analysis with the proteome. Assesment of DOX-induced effects using our scoring system, showed the ability to identify toxicant-induced changes in miRNA-circRNA regulation of translation inhibition. Finally, we were also able to estimate the amount of translatable mRNAs, which improved the correlation with the proteome. This suggests that assessment of toxicological processes through transcriptomics analysis would benefit from the assessment of post-transcriptional mechanisms. 


\section{Methods}

\section{Samples}

Human 3D cardiac microtissues (InSphero, SWL), composed of induced pluripotent stem cells (iPSC)-derived human cardiomyocytes (female Caucasian donor) and cardiac fibroblasts (male Caucasian donor) in a ratio of 4:1 (approximately 5000 cells per microtissue), were used. 3D Insight ${ }^{\mathrm{TM}}$ Human Cardiac Microtissue Maintenance Medium (InSphero, Cat \#CS-07-010-01) was used to culture the microtissues.

\section{PBPK model establishment}

The physiologically based pharmacokinetic (PBPK) modelling software PK-Sim was used as previously described ${ }^{[34]}$ to establish the PBPK dosing profile resembling physiologically relevant exposure levels of DOX. Interstitial heart concentrations after administration of a daily therapeutic DOX dose $\left(0.415 \mathrm{mg} \cdot \mathrm{kg}^{-1}\right)$ were estimated. The obtained concentration profiles were thereafter converted into discrete daily exposure profiles to be applied to the cardiac cell model.

\section{Exposure}

The PBPK-based repetitive dosing profile described above was realized by means of three medium changes per day, with the addition of DOX stock solution (Sigma D1515, Cat \#25316-40-9, dissolved in DMSO) to achieve the PBPK-based incubation concentration of DOX. As control, microtissues exposed to similar end concentrations of DMSO were used. Samples were obtained in triplicate at 2, 8, 24 and 72 hours of DOX exposure. Each individual sample contained a pool of 36 microtissues (incubated separately) for RNA isolation using Qiagen AllPrep DNA/RNA/miRNA Universal Kit (Cat \#80224) and a pool of 18 microtissues was used for protein isolation through freezethaw cycles followed by centrifugation. 


\section{RNA sequencing}

Ribosomal depletion of the isolated total RNA was accomplished using the Illumina RiboZero Gold kit (Cat \#MRZG12324). Thereafter, the samples were prepared for sequencing (HiSeq2500, 100bp paired-end) using the Lexogen SENSE total RNA library preparation kit (Cat \#009.96). All 8 lanes of a flow cell contained a pool of all DOX samples, resulting in an average sequencing depth of 20.3 million raw reads per sample. The same was done for the control samples, which were sequenced with an average of 51.2 million raw reads per sample. Lexogen adapter sequences were removed from the reads using Trimmomatic version $0.32^{[35]}$ to trim the first 12 bases of the $5^{\prime}$ end. FastQC version $0.11 .3^{[36]}$ used to assess the quality of the sequencing data before and after trimming.

\section{Messenger RNA analysis}

Alignment of the sequencing reads to the Ensembl reference genome (Genome Reference Consortium Human Build 38, GRCh38.p7) was done using RSEM ${ }^{[37]}$ (v.1.2.28) with the paired-end and Bowtie2 ${ }^{[38]}$ (v.2.2.6) option. The output included TPM (transcripts per million) normalized read counts for each transcript which were used for analysis.

\section{CircRNA analysis}

Prediction of circRNAs was done using find_circv1.2 ${ }^{[39]}$. Sequences which could not be mapped onto the Ensembl reference genome (Genome Reference Consortium Human Build 38, GRCh38.p7) were used to obtain anchors of 20bp at both read ends. Exon splice sites were identified by realignment of the individual anchors to the genome. Additional to the authors' criteria for obtaining circRNAs, a minimum quality score of $35 \mathrm{bp}$ was used for both anchors, as described by Caiment et al. ${ }^{[40]}$. This resulted in the chromosomal location of the circRNA which were used to retrieve sequences with the BioMart R package ${ }^{[41]}$. Finally, RSEM was used to map the sequencing reads to the circRNA sequences to obtain TPM (transcripts per million) normalized read counts for each circRNA which were used for analysis. 


\section{MiRNA sequencing}

Of the isolated total RNA, an aliquot was used for size selection and ligation using the TruSeq Small RNA Library Prep Kit (Illumina ${ }^{\circledR}$ ). After sequencing on the HiSeq2500, adapter sequences were removed and reads between 16 and $35 \mathrm{bp}$ in length were analysed. As described previously ${ }^{[42]}$, reads were aligned to the human genome using PatMaN $^{[43]}$ (v.1.2.2). No gaps or mismatches were allowed during this alignment. Finally, the mapping output was parsed to obtain complete read counts for $3^{\prime}$ and 5' miRNA species which were used for analysis.

\section{Interaction prediction}

MicroRNA interactions with mRNAs or circRNA (Figure 1) were predicted using miRanda ${ }^{[44]}$. As input, all sequenced miRNAs and identified circRNA sequences were used (not taking into account differential expression), combined with all known human 3'UTR mRNA sequences obtained through BioMart. By selecting miRanda scores $>140$, only perfect matches (not allowing gaps or wooble pairing) of a seed region were included as a predicted interaction.

\section{Proteomics}

Proteomics analysis performed as described in chapter 2 . Isolated proteins were diluted to a concentration below 0.2M.Peptide digestion was achieved using trypsin and Sep-Pak tC18 cartridges (Waters) were used for peptide clean-up according to the manufacturer's instructions. Peptides were dried using a vacuum centrifuge before measurement on a Orbitrap Fusion mass spectrometer (Thermo Fisher Scientific) coupled to a NanoLC-2D HPLC system (Eksigent). Processing of raw MS data was performed using the Genedata Expressionist software (v.11.0). After noise reduction and normalization, properties of LC-MS peaks were obtained ( $\mathrm{m} / \mathrm{z}$ and RT boundaries, $\mathrm{m} / \mathrm{z}$ and RT center values, intensity). Mascot 2.6 was used for annotation of the individual MS/MS spectra. Protein interference was used to group peak clusters (based on peptide and protein annotations) and protein intensities were computed using the Hi3 method ${ }^{[45]}$. Thereafter, data was log2 transformed and each sample was shifted to the median of the medians of all samples (all DOX samples + all DMSO samples). 


\section{Scoring system: miRNA inhibition of mRNA translation}

In order to quantify interactions between mRNA, miRNA and circRNA, we designed a scoring system which reflects the magnitude of miRNA inhibition of translation (MIT). The scoring system is based on a calculation which can be applied to a single transcript of interest or genome-wide to all transcripts of a sample sequentially. Essentially, for every transcript, the miRNAs predicted to interact are obtained using miRanda.

Thereafter the scoring system is applied to all of these miRNAs. To cover the biological mechanism of miRNA-circRNA regulation, the scoring system contains three elements: 1) the miRNA efficiency, 2) the circRNA efficiency and 3) combined miRNA and circRNAs effect.

The efficiency of a single miRNA ( $E_{\text {miRnA }}$; formula 1 ) is dependent on the abundance of the miRNA ( $\operatorname{miRN} A_{\text {count }}$ ) and the amount of seed regions it can interact with in the target mRNA ( $\left.m R N A_{\text {seeds }}\right)$. The strength of translation inhibition increases with the number of seed regions, which is represented in the formula by an exponential function. The number of seed regions is obtained through prediction of interaction between a miRNA and its target mRNA using miRanda with strict parameter settings. Within the calculation, we assume all seed regions are involved during inhibition of translation:

Formula 1

(for every miRNA)

$$
E_{\text {miRNA }}=\operatorname{miRN} A_{\text {count }} \operatorname{mRNA}_{\text {seeds }}
$$

Because miRNAs can be prevented to interact with mRNA by circRNAs, the efficiency of capturing miRNAs by circRNAs $\left(E_{\text {circRNA }}\right)$ also was estimated. Using miRanda, we retrieved the circRNAs which are predicted to interact with a single miRNA $\left(n_{\text {circRNA }}\right)$. Most ideal would be to multiply the circRNA abundance $\left(\operatorname{circRNA} A_{\text {count }}\right)$ with its number

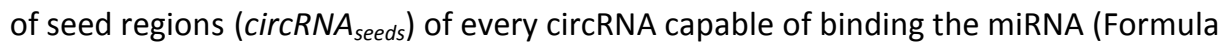
2a). This, however, greatly increased the run-time complexity and therefore the calculation time when applied genome-wide. To reduce this time complexity of the 
algorithm, we proceeded with an acceptable approximation which comprised of the multiplication of the total circRNA abundance $\left(\operatorname{circRN} A_{\text {count }}\right)$ with the average of seed regions within these circRNAs (Formula $2 b$ ):

Formula $2 a$

(for every miRNA)

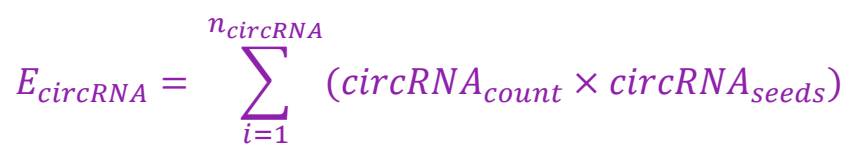

Formula $2 b$

(for every miRNA)

$$
E_{\text {circRNA }}=\sum_{i=1}^{n_{\text {circRNA }}} \operatorname{circRN} A_{\text {count }} \times \frac{\sum_{i=1}^{n_{\operatorname{circRNA} A}} \operatorname{circRN} A_{\text {seeds }}}{n_{\text {circRNA }}}
$$

Thereafter, the interplay between miRNA and circRNAs was calculated using formula 3. Essentially, this formula represents the fraction of miRNAs which are not captured by circRNAs (or "free" miRNAs). In order to prevent a division by 0 in the absence of any circRNAs capable of capturing a given miRNA, we added 1 to the circRNA term. This will not have biological implications, because the circRNA term typically ranges from $1 \cdot 10^{4}$ to $1 \cdot 10^{12}$ when circRNAs are expressed. When no circRNAs are expressed, the circRNA becomes negligible and the miRNA term determines the outcome of the fraction. By taking the inverse of this fraction, the magnitude of miRNA inhibition of translation (MIT) is obtained, in which 0 represents no miRNA inhibition and 1 complete inhibition:

Formula 3

(for every miRNA)

$$
M I T=1-\left(\frac{E_{\operatorname{circRNA}}+1}{E_{\text {miRNA }}+\left(E_{\operatorname{circRNA}}+1\right)}\right)
$$

Integrating individual parameters 1-3 results in the following complete scoring system:

Formula 4 (for every miRNA)

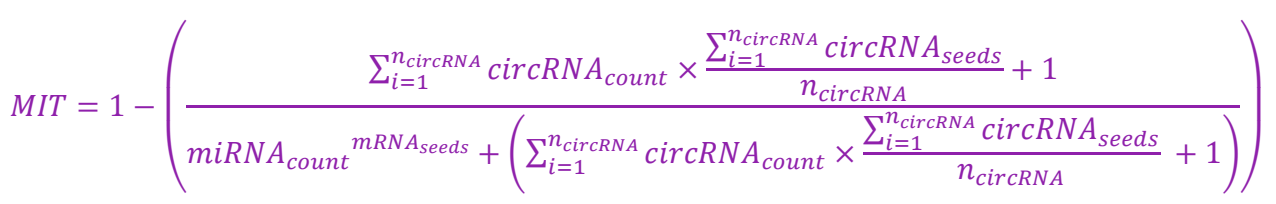




\section{Results}

In order to assess whether DOX is able to induce changes in miRNA-circRNA regulation, we exposed human cardiac microtissues to DOX up to 72 hours. We exposed the microtissues to physiologically based pharmacokinetic (PBPK)-based dosing profile which mimics the in vivo DOX concentrations in a patient's heart during daily therapeutic DOX treatment able to induce cardiotoxic side-effects. To realize the fluctuation of the PBPK-based DOX concentrations, medium of the microtissues was changed three times daily. Samples were obtained at 2, 8, 24 and 72 hours of DOX exposure. Each sample was analysed using proteomics and transcriptomics techniques.
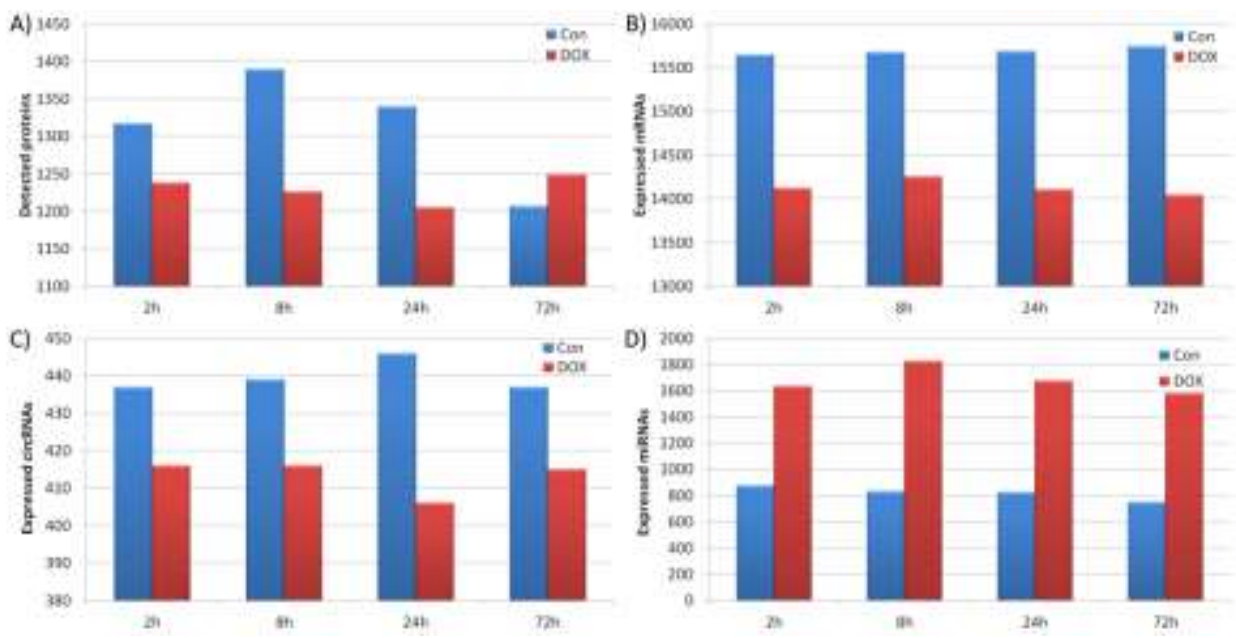

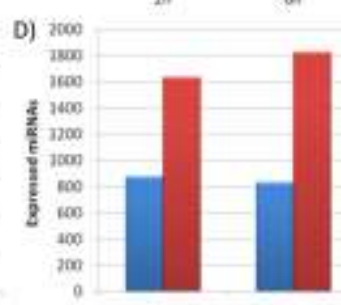

20

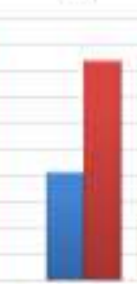

1 an

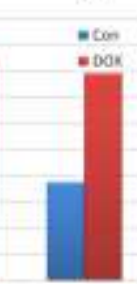

mh

Figure 1: Numbers of detected entities at each time point for DOX-exposed (red) and control samples (blue). Depicting the amount of A) detected proteins, B) expressed mRNAs, C) expressed circRNAs, and D) expressed miRNAs. To obtain a complete overview of miRNA-circRNA regulation, no cut off criteria based on differential expression were applied

The proteomics dataset contained on average $1230 \pm 19$ detected proteins per time point for DOX-exposed samples and $1314 \pm 77$ detected proteins per time point for control samples (Figure 1A). The transcriptomic dataset was made up of mRNAs, circRNAs and miRNAs. In DOX-exposed samples, 14134 $\_88$ mRNAs, $413 \pm 5$ circRNAs and 1679 +106 miRNAs were expressed at each time point; and control samples indicated expression of 15690 \pm 40 mRNAs, $440 \pm 4$ circRNAs and 823 \pm 52 miRNAs (Figure 1B-D). We included all detected entities in our analysis (no selection on differential expression) because we assume that a non-differential change for miRNAs and/or 
circRNAs has the ability to include a differential change in protein abundance. The amount of detected mRNAs, circRNAs and proteins was higher for control samples as compared to DOX-exposed samples, except for proteomics measurement at $72 \mathrm{~h}$ of exposure. Furthermore, in DOX-exposed samples more miRNAs were detected as compared to control samples.

\section{The scoring system}

\section{niRNA-circRNA interactions}

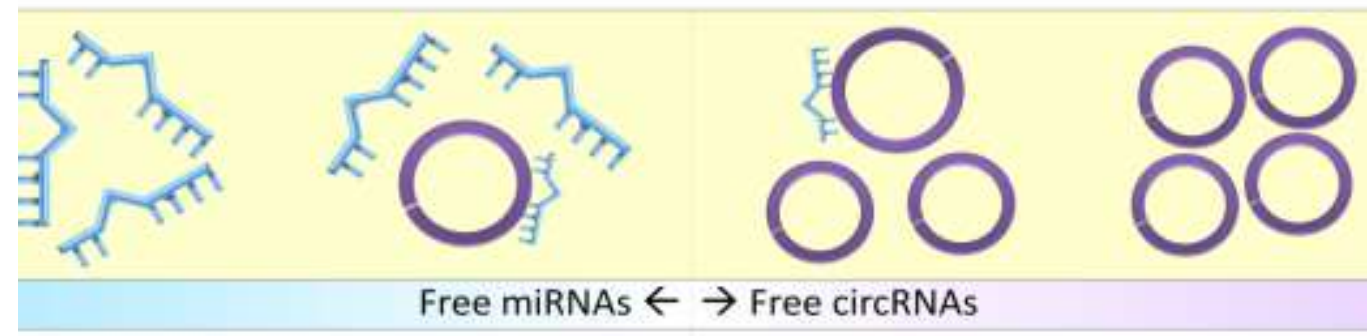

\section{niRNA-mRNA interactions}

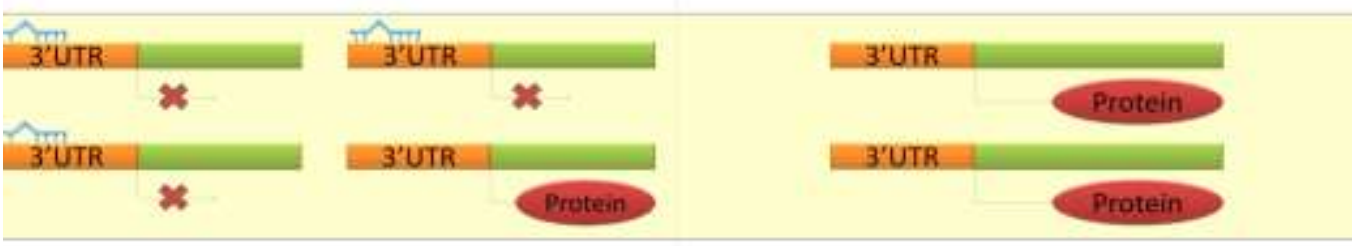

\section{Igorithm}

\section{ИIT score}

\section{mRNA translatior}

Figure 2: Visualization of the basis of the scoring system. Depending on the amount of miRNA (blue) and circRNA (purple) present within a cell, interactions between them can occur, resulting in captured and free miRNAs and circRNAs. When there are more "free" miRNAs, translation of more mRNAs can be inhibited. Therefore, the algorithm will return a higher MIT score in these cases

In this pilot study, we designed a scoring system based on the expression values and predicted interactions of mRNA, miRNA and circRNA (see Methods) for the assessment of posttranscriptional miRNA-induced inhibition of translation (MIT). In short, for every mRNA, the miRNAs predicted to interact were obtained (using miRanda). For each of these interacting miRNAs, a MIT score was calculated using the scoring system. The scoring system assessed the interaction between miRNAs and circRNAs (using miRanda) 
and predicted the fraction of miRNAs which were not captured by circRNAs. This fraction of uncaptured or "free" miRNAs was theoretically capable of inhibiting translation through binding to its seed region within the 3'UTR of the target mRNA (Figure 2). The calculated MIT score ranged from 0 (= no "free" miRNAs = no inhibition) to 1 (= only "free" miRNAs = complete inhibition).

\section{Detailed demonstration of our scoring system}

In the current section we demonstrate step by step how the scoring system works, how it was designed and how it can be used to identify changes in miRNA-circRNA regulation. For this purpose we selected the $A P O B E C 2$ transcript as a case study. This transcript was selected because it revealed the most DOX-induced changes in MIT scores during the genome-wide application of the scoring system.

The selected example transcript, APOBEC2 (ENSG00000124701), encodes the C->UmRNA editing enzyme APOBEC-2 (Q9Y235). Using miRanda, 38 miRNAs were predicted to interact with the $A P O B E C 2$ transcript through perfect base paring of the seed region (meaning that gaps and wobble base pairing were not allowed). However, not all of the

38 miRNAs were expressed in every sample. Figure $3 A$ shows that on average 22 different miRNAs were expressed in control samples, while in DOX-exposed samples on average 32 miRNAs were detected. Even though the number of expressed miRNAs was higher in DOX-exposed samples, the sum of expressions of all miRNA abundances of was lower compared to control samples (Figure 3B). This indicated a decrease in expression for several or all of the miRNAs in DOX-exposed samples.

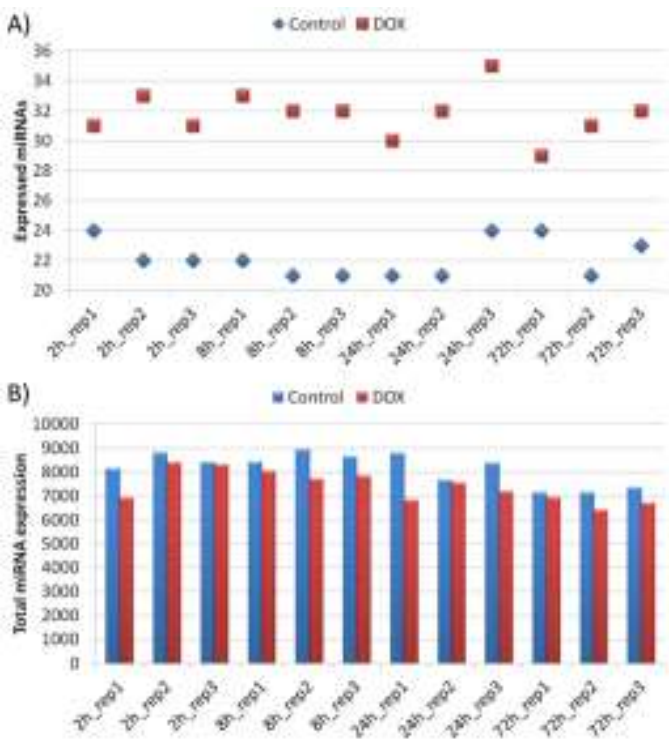

Figure 3: MiRNAs interacting with the APOBEC2 transcript. A) Depicts the number of expressed miRNAs, and B) total expression values of these miRNAs (sum of all expressed miRNA). Red $=$ DOX exposed samples, Blue $=$ control samples 
For each of the 38 miRNA, also the predicted interactions with circRNAs identified in our experimental data were obtained by using miRanda. The miRNA with the least interactions was predicted to be captured by 63 circRNAs, while the miRNA with the most interactions could be captured by 249 detected circRNAs according to the predictions. For every miRNA interacting with the $A P O B E C 2$ transcript, for every sample at every time point, these interactions were assessed using the scoring system.

\section{Example of our scoring system: calculation of MITMax score for APOBEC2}

For comprehensive understanding of the MIT score calculation, we discuss miR-409$3 p$ in more detail, because this miRNA depicted the biggest change in MIT scores between DOX-exposed samples and control samples. Complete calculations are displayed for the $8 \mathrm{~h}$ rep3 sample. Other values are depicted in Figure 4.

We start our demonstration by assessing the effects miR-409-3p can have on the $A P O B E C 2$ transcript. The first factor of importance was the expression level, which was strongly decreased after DOX-exposure, while it was highly expressed in the control samples (Figure 4A). Furthermore, the amount of seed regions in the 3'UTR was important. Most miRNAs interact with a transcript through 1 seed region. When a single miRNA interacts though multiple seed regions, as is the case for miR-409-3p $(n=2)$, the efficiency greatly increases. Therefore, miRNA expression value to the power of the amount of seeds, represented the miRNA efficiency $\left(E_{\text {miRnA }}\right)$ to inhibit translation:

$$
\begin{gathered}
E_{\text {miRNA }}^{\text {DOX_8 } \_ \text {rep } 3}=\text { miRNA }_{\text {count }}{ }^{\text {RNNA }_{\text {seeds }}}=86.499^{2}=7482.06 \\
E_{\text {miRNA }}^{\text {Con_8h_rep3 }}=936.547^{2}=877121
\end{gathered}
$$

Due to the lower expression level of miR-409-3p in DOX-exposed samples, also its efficiency was greatly decreased compared to control samples (Figure 4B-C). This decrease was found for all DOX-exposed samples. 


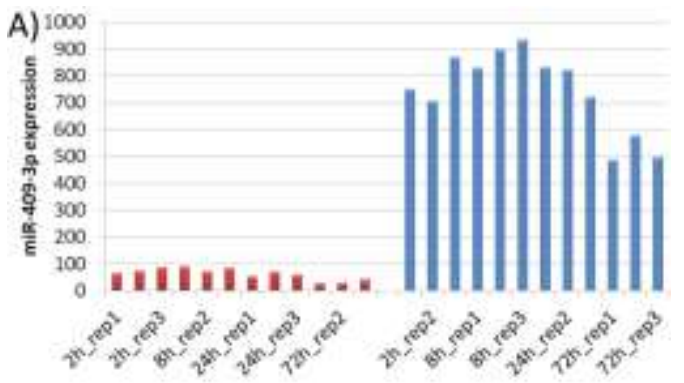

Figure 4: Analysis of miR-409-3p. DOX exposed samples depicted in red and control samples in blue.

A) Expression levels of miR-409-3p. B) DOX miRNA efficiency values. C) Control miRNA efficiency values.

D) circRNA efficiency values. E) MIT scores of miR-409-3p.

F) Expression of circRNAs (the height of the bar represents the total expression value; Pink = expression of the circRNA chr14: 20343146-20343277; Blue = expression of chr22: 35610883-35611106. G) Graph E zoomed in by exclusion of the highly expressed (chr14:

B) 20343146-20343277)
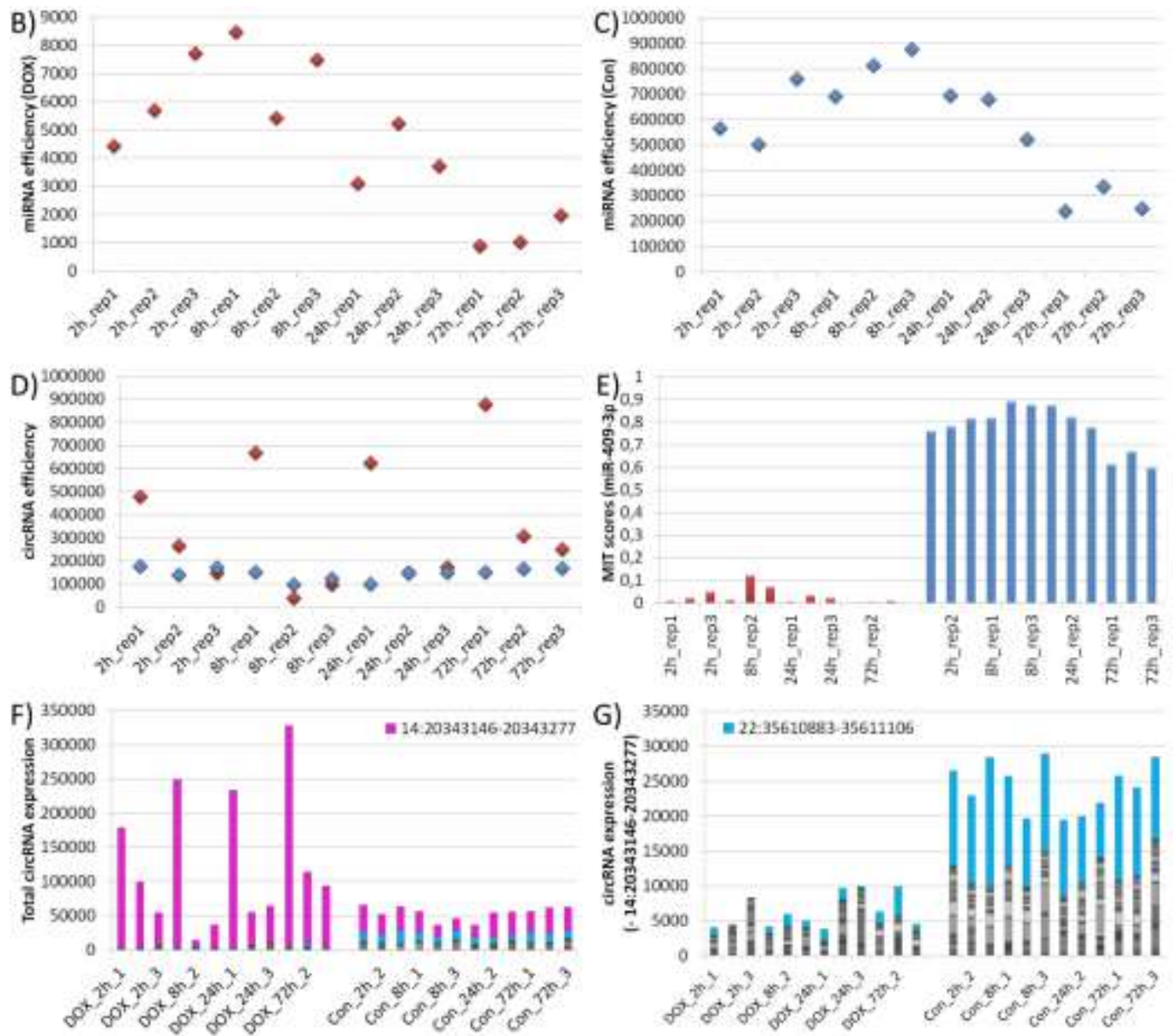
The next step was to access the predicted efficiency of circRNAs to capture miR409-3p ( $\left.E_{\text {circRNA }}\right)$. Using miRanda, 164 circRNAs were predicted to capture miR-409-3p through a total of 439 seed regions in these circRNAs. To obtain the circRNA efficiency, the total expression of these 164 circRNAs (Figure 4F) was multiplied by the average of seed regions:

$$
\begin{gathered}
i_{\text {circRNA }}^{\text {DOX_8h_rep } 3}=\sum_{i=1}^{n_{\text {circRNA }}} \operatorname{circRNA} A_{\text {count }} \times \frac{\sum_{i=1}^{n_{\text {circRNA }}} \operatorname{circRNA} A_{\text {seeds }}}{n_{\text {circRNA }}}=36449.29 \times \frac{439}{164} \\
=97568.53 \\
i_{\text {circRNA }}^{\text {Con_8h_rep } 3}=45858.96 \times \frac{439}{164}=122756.6
\end{gathered}
$$

These calculations reveal a DOX-induced decrease in circRNA efficiency due to a decrease in total circRNA expression for the 8h_rep3 sample. However, when assessing all DOX-induced samples, the total circRNA expression was highly variable with most time points indicating an increase in circRNA expression. Therefore, the circRNA efficiency in DOX-exposed samples was also variable and indicated increased efficiency at most time points (Figure 4D). Deeper analysis into individual circRNAs identified 14:20343146-20343277 \& 22:35610883-35611106 as major influencers of the circRNA efficiency value. First, the total expression of the circRNAs was dominated by the expression values of 14:20343146-20343277 (pink, Figure 4F). Especially in DOXexposed samples the expression of this circRNA was extremely variable, which corresponded to the variability of the circRNA efficiency values. Second, the most significant change $\left(p=9.5 \cdot 10^{-12}\right)$ was observed for 22:35610883-35611106, with a strong decrease after DOX exposure (blue, Figure 4G). Overall, in DOX-exposed samples, the 14:20343146-20343277 circRNA was predicted to be almost solely responsible for capturing of miR-409-3p. In control samples, miR-409-3p was captured by both $14: 20343146-20343277$ and 22:35610883-35611106. 
In the final step we combined the miRNA and circRNA efficiencies. By dividing the total circRNA efficiency by the sum of the miRNA and circRNA efficiencies, the fraction of captured miRNAs was obtained. Because the obtained value was inversely correlated with the amount of non-captured or "free" miRNAs which interact with mRNAs, the inverse of the obtained value constituted the MIT score (miRNA inhibition of translation):

$$
\begin{aligned}
& M I T^{\text {DOX }_{8 h_{\text {rep } 3}}}=1-\left(\frac{E_{\text {circRNA }}+1}{E_{\text {miRNA }}+\left(E_{\text {circRNA }}+1\right)}\right)=1-\left(\frac{97568.53+1}{7482.06+(97568.53+1)}\right) \\
& =0.0712 \\
& M I T^{\text {Con_8h_rep } 3}=1-\left(\frac{122756.6+1}{877121+(122756.6+1)}\right)=0.8772
\end{aligned}
$$

For 8h DOX exposure ( $3^{\text {rd }}$ replicate), a MIT score of 0.0712 was obtained. This theoretically indicates that $7 \%$ of transcripts were subjected to translation inhibition. Contrastingly, the corresponding control sample resulted in a MIT score of 0.8772 , indicating a major inhibition of $A P O B E C 2$ translation with $88 \%$ of transcripts possibly not being translated due to miR-409-3p interactions. This same pattern was observed for the other samples (Figure 4E), indicating a major DOX-induced decrease of miRNA translation inhibition.

We have now shown the calculation of the MIT score for a single miRNA in a single sample at a single time point for both DOX-exposed samples and control samples. As stated before, MIT scores were obtained for every miRNA interacting with the $A P O B E C 2$ transcript, for every sample and at every time point, resulting in $381 \mathrm{MIT}$ scores for DOX-exposure and 268 for the control condition (Figure 5).

Figure 5 shows that most of the obtained MIT scores are below 0.025 , implying that individual miRNAs only had a minor influence on translation inhibition. High MIT scores (>0.6) were only obtained for control samples. These high values were gained from miR-409-3p used in the calculations above. 


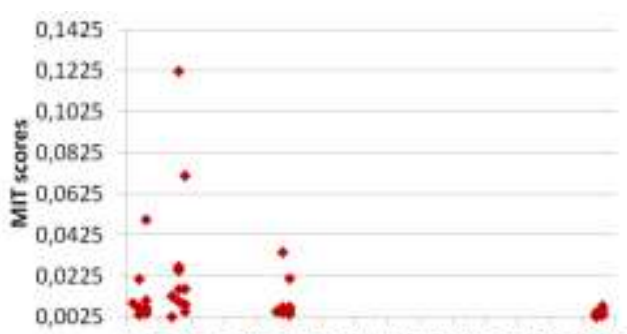

$0 \quad 51015202530354045505560657075$
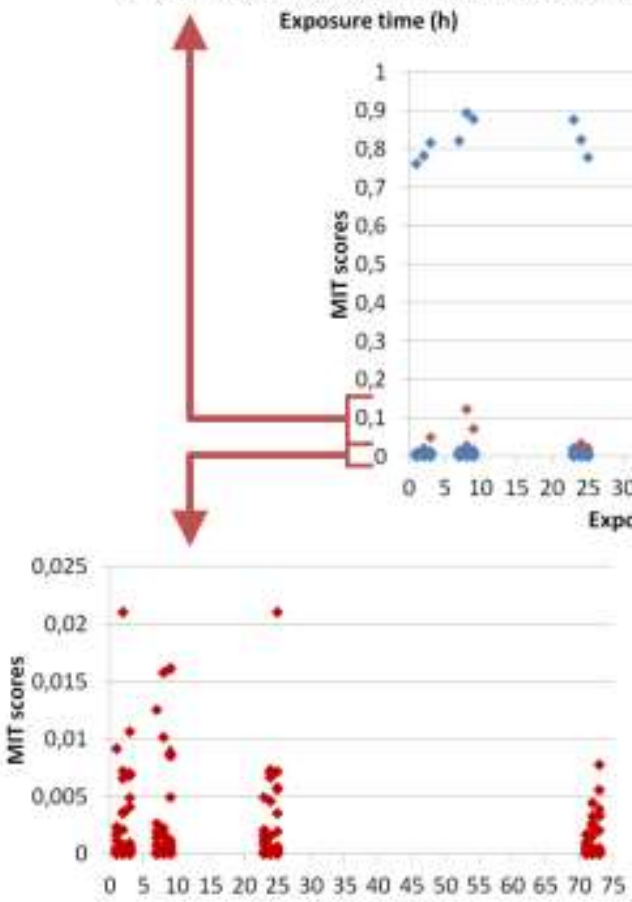

Exposure time (h)
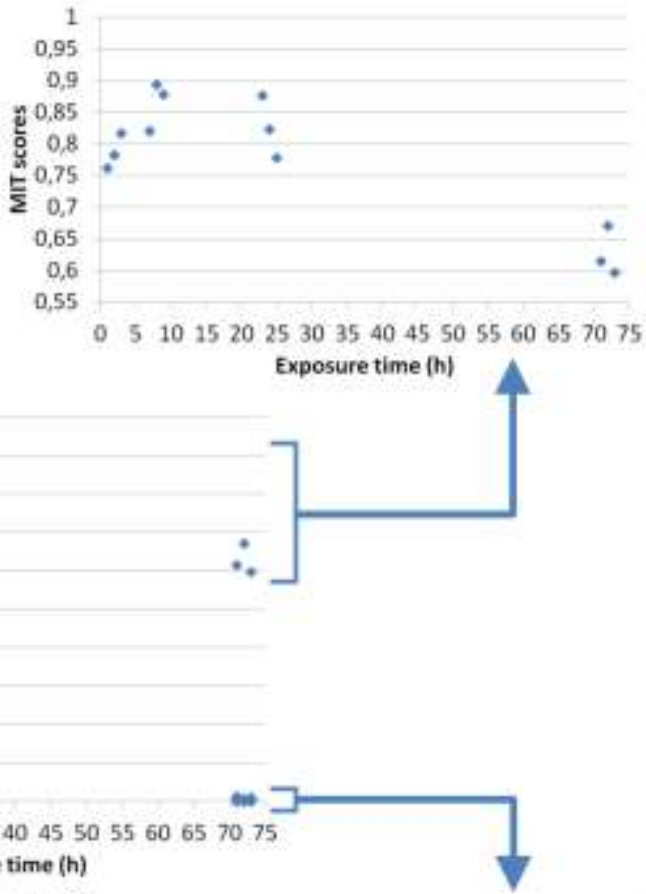

Exposure time (h)

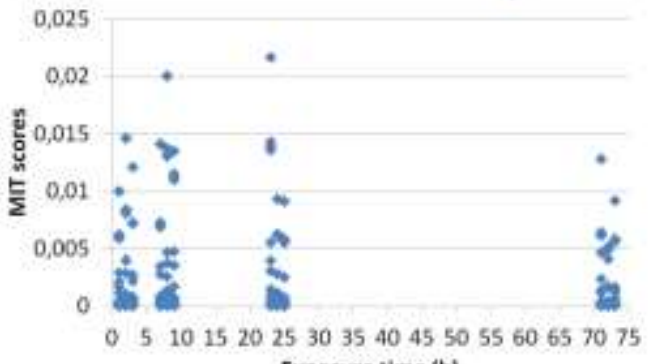

Exposure time (h)

Figure 5: APOBEC2 MIT scores of all miRNAs at every time point. In the centre is the complete graph of the MIT score depicted, with DOX-exposed samples in red and control samples in blue. The x-axis represents the exposure time. Due to the variation within the MIT scores, parts of the graph are enlarged to increase visibility. The top graphs represent the highest obtained MIT scores, with DOX-exposure on the left and control samples on the right (note that these 2 graphs have different y-axis values). Furthermore, the bottom graphs represent the lowest obtained MIT scores, with DOX-exposure on the left and control samples on the right (y-axis values identical) 


\section{Interpretation of MIT scores}

In order to draw conclusions regarding toxicant-induced changes in miRNA-circRNA regulation, we needed to interpret the multitude of obtained MIT scores. In the current pilot study, we used two concepts to assess the translation inhibition per transcript:

1. MIT ${ }^{\text {Mean }}$ : the mean inhibition score of the expressed miRNAs. We selected $\mathrm{MIT}^{\text {Mean }}$ because this represents the sum of all MIT scores, normalized to a 0-1 scale. 2. $\mathrm{MIT}^{\mathrm{Max}}$ : the maximum inhibition score. We selected $\mathrm{MIT}^{\mathrm{Max}}$ because we postulate that the miRNA with the maximum MIT score determines most of the biological translation inhibition while the effect of other miRNAs might be negligible in comparison.

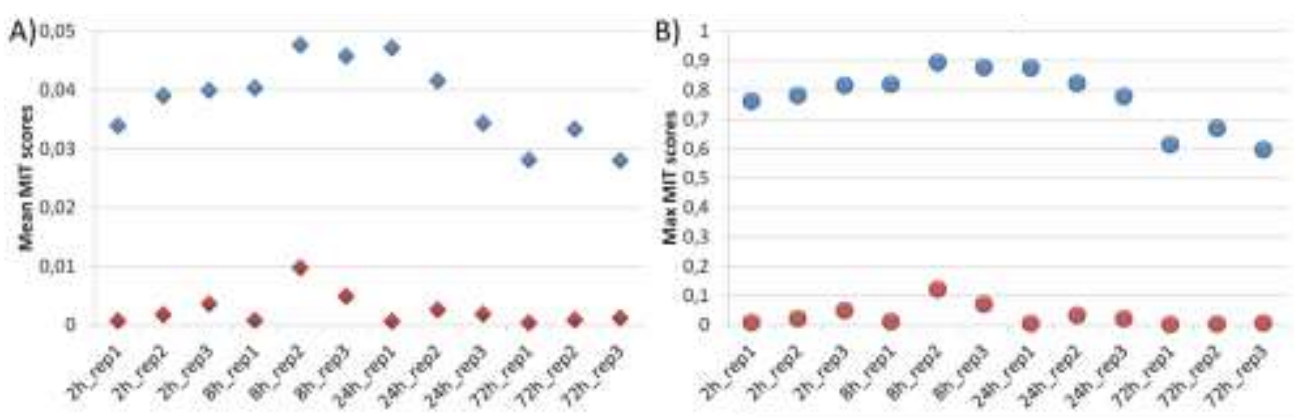

Figure 6: MIT scores obtained for miRNAs interacting with the APOBEC2 transcript. DOX exposed samples are depicted in red and control samples in blue. A) MIT ${ }^{\text {Mean }}$ scores are depicted with diamonds, and B) MIT ${ }^{\mathrm{Max}} \mathrm{Scores}$ are depicted with circles

For the APOBEC2 transcript, both the $\mathrm{MIT}^{\mathrm{Mean}}$ (Figure 6A) and the $\mathrm{MIT}^{\mathrm{Max}}$ scores (Figure 6B) clearly revealed the decrease of MIT scores for DOX-exposed samples compared to the control samples, even though the absolute values were very different from each other. This may indicate that both parameters are equally capable of representing toxicant-induced changes in miRNA-circRNA regulation.

To further evaluate the usefulness of the $\mathrm{MIT}^{\mathrm{Mean}}$ and $\mathrm{MIT}^{\mathrm{Max}}$ scores, we also assessed results gained from other transcripts. Included in the analysis were expressed transcripts (TPM > 1) for which MIT scores were obtained in at least 2 replicates of 1 time point of DOX exposure and its time matched control. This resulted in 9803 
transcripts (Figure 7) of which 5700 were found at all time points. These inclusion criteria were set in order to obtain reliable MIT scores for the included transcripts, which are necessary for the demonstration of the scoring system. Consequently, 2 groups which could be of interest when elucidating toxicological processes were excluded: 1 ) Transcripts completely inhibited by DOX exposure ( $n=20$, only detected in control), and 2 ) Transcripts activated by DOX exposure ( $n=782$, not detected in control).

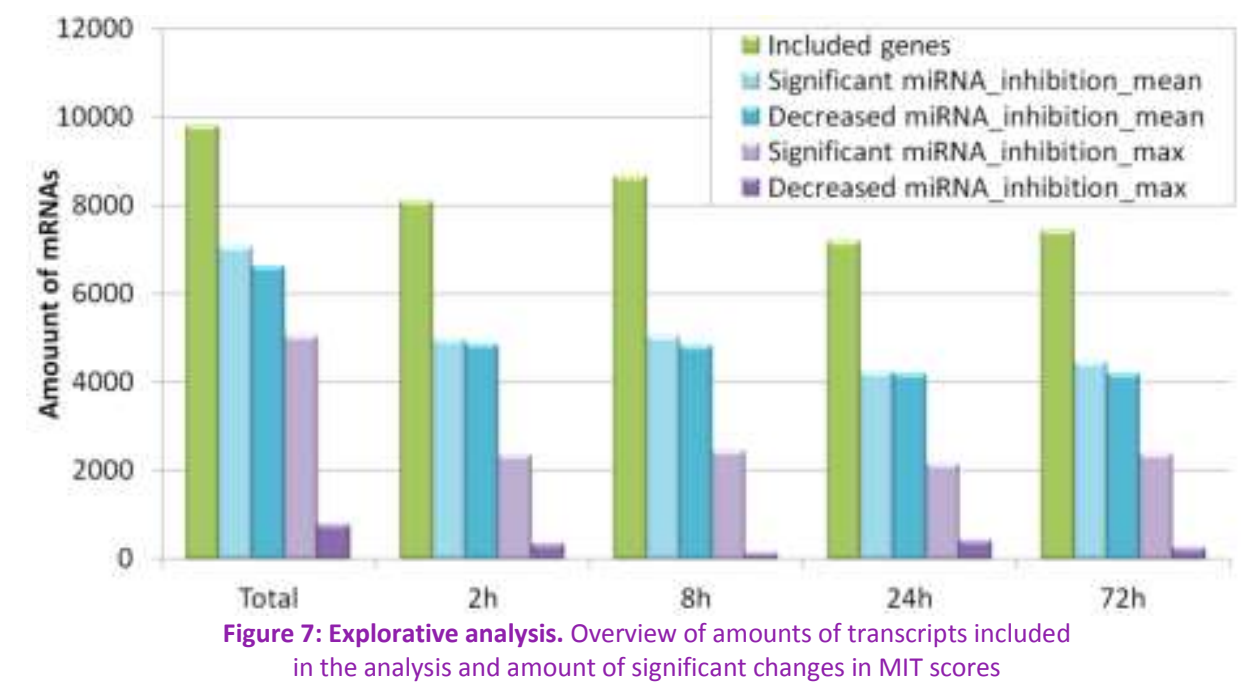

Comparison of the calculated $\mathrm{MIT}^{\mathrm{Mean}}$ score between DOX-exposed and control samples revealed significant changes (students T-test $\mathrm{p}<0.05$ ) for approximately $60 \%$ of analysed transcripts per time point (Figure 7). Most (94-99\%) of these significant changes revealed a decreased $\mathrm{MIT}^{\text {Mean }}$ score after DOX exposure. However, when analysing the calculated $\mathrm{MIT}^{\mathrm{Max}}$ score, approximately $30 \%$ of transcripts were found significantly changed per time point, of which $<10 \%$ were decreased in DOX exposure compared to control. This may indicate that DOX decreased the overall inhibition capability of most miRNAs (as predicted by the decreased $\mathrm{MIT}^{\text {Mean }}$ score), while several miRNAs were regulated differently resulting in an increase of inhibition capability (as predicted by the increased $\mathrm{MIT}^{\mathrm{Max}}$ score). While these observations indicated that DOX was able to induce changes in miRNA-circRNA regulation of translation, this also showed a fundamental difference between the MIT ${ }^{\text {Mean }}$ and the $\mathrm{MIT}^{\mathrm{Max}}$ scores. To verify biological relevance of these parameters, we validated these parameters against the proteome. 


\section{MIT scores used to obtain translatable mRNA read counts}

Because the MIT score represents the fraction of miRNAs which can inhibit translation, we were able to use the inversed value to calculate the translatable read count (TRC) of transcripts. These TRCs can thereafter be used for correlation analysis with the proteome. To illustrate, we return to the example of the APOBEC2 transcript inhibited by miR-409-3p (which constitutes the MIT ${ }^{\mathrm{Max}}$ score):

$$
\begin{gathered}
T R C^{D O X_{\_} 8 h_{-} r e p 3}=m R N A_{\text {count }} \times(1-M I T)=95.02 \times 0.9287=88.25 \\
T R C^{\text {Con_8 } h_{-} r e p 3}=58.26 \times 0.1228=7.15
\end{gathered}
$$

For control samples, the number of translatable mRNAs was drastically decreased after the adjustment for inhibiting miRNAs. In contrast, in DOX-exposed samples, the adjustment hardly changed the expression values due to low $\mathrm{MIT}^{\mathrm{Max}}$ scores (Figure 8B).

\section{Validation of MIT scores: Correlation of TRC with the proteome (control samples)}

We assessed whether the MIT ${ }^{\text {Mean }}$ or the $\mathrm{MIT}^{\mathrm{Max}}$ best reflected the biological mechanism of translation regulation, through Pearson correlation analysis between the proteome and TRCs obtained using $\mathrm{MIT}^{\text {Mean }}\left(\mathrm{TRC}^{\text {Mean }}\right.$ ) or $\mathrm{MIT}^{\mathrm{Max}}\left(\mathrm{TRC}^{\mathrm{Max}}\right)$. A biologically relevant scoring system was identified through improvement of the correlation in control samples.

First, we discuss the $A P O B E C 2$ example transcript. The correlation between APOBEC-2 protein abundance (Figure 8A) and unadjusted RNA (Figure 8B, RNA_TPM) resulted in a correlation coefficient ( $r$ ) of 0.768 (Figure $8 \mathrm{C}$ ). Compared to this coefficient, the correlation improved only slightly when using the $\operatorname{TRC}^{\text {Mean }}(r=0.775$, which was not surprizing given the low $\mathrm{MIT}^{\text {Mean }}$ values and $\mathrm{TRC}^{\text {Mean }}$ values (green triangles) highly similar to unadjusted RNA counts (red squares). However, a notable improvement was observed using $\operatorname{TRC}^{\mathrm{Max}}(r=0.840)$. Due to high MIT $^{\text {Max }}$ values, the $T_{R C}{ }^{\text {Max }}$ values (blue circles) drastically decreased compared to unadjusted RNA counts. Furthermore, the pattern of the TRC ${ }^{\mathrm{Max}}$ values closely resembled the pattern of protein 


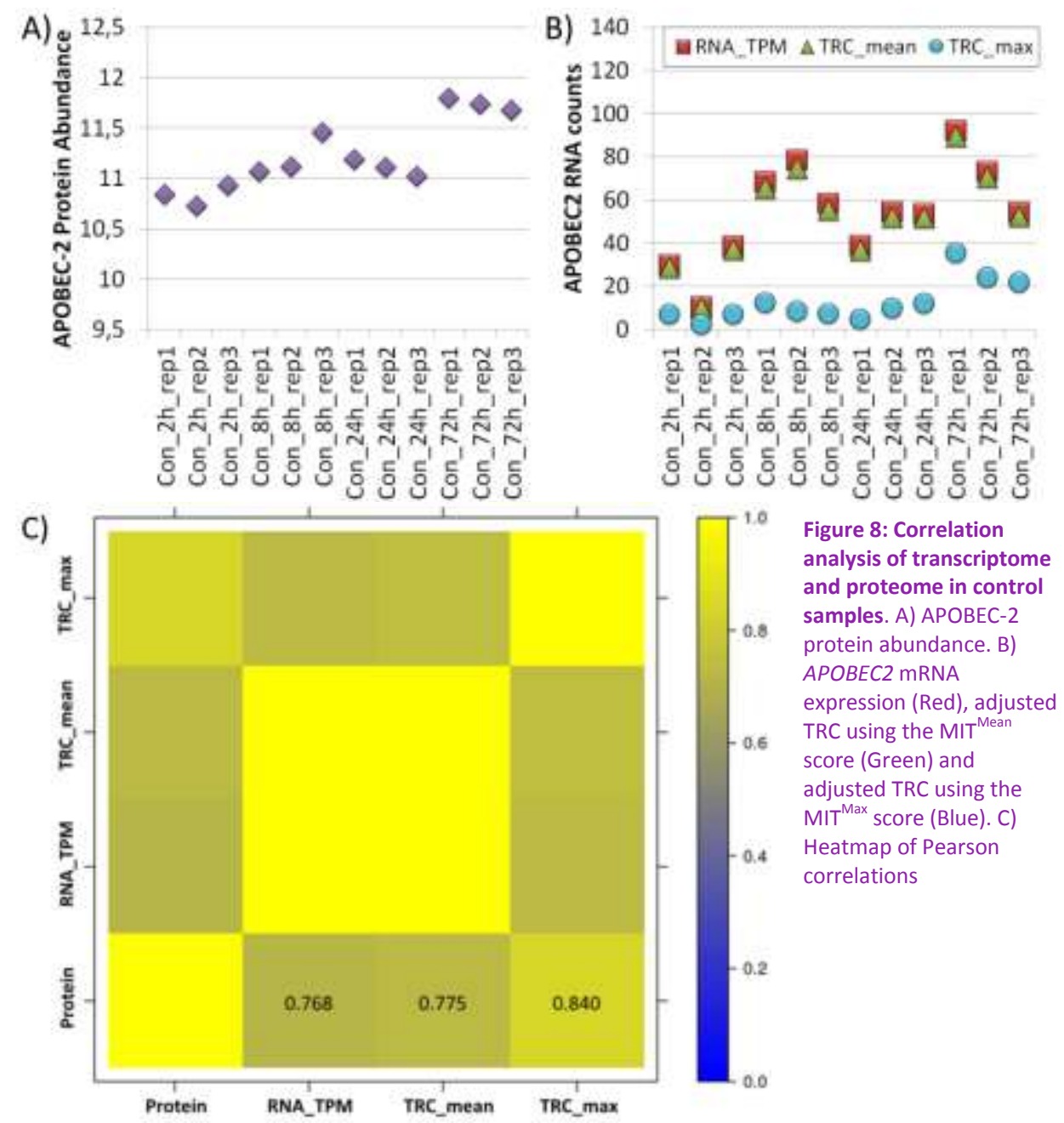

abundance values. Because of this agreement between the proteome and TRC ${ }^{\mathrm{Max}}$, we can conclude that the miRNA with the maximum MIT score determined most of the biological translation inhibition for the $A P O B E C 2$ transcript and is therefore the best parameter to be used in our scoring system.

Second, to evaluate whether the conclusions regarding $\mathrm{MIT}^{\mathrm{Max}}$ can be generalized to other transcripts, we expanded the correlation analysis by using 40 protein-transcript sets. Used selection criteria were: 1) significant DOX-induced changes in both MIT ${ }^{\text {Mean }}$ and $\mathrm{MIT}^{\mathrm{Max}}$ scores at all time points, and 2) the availability of proteomics data at all time points. 
The Pearson correlation of these 40 proteins with their corresponding RNA counts and TRCs were assessed (Supplementary Table I). Cases in which the TRC resulted in a correlation shifted towards positive 1 compared to unadjusted RNA values ( $r^{T R C}$ $r^{T P M}=$ positive) were considered as an improvement of the correlation (even when the absolute coefficient was negative).

Use of TRC Mean resulted in 47.5\% (19 of 40) improved correlations in the control samples. As expected, the absolute increases were very small, with none of the changes larger than 0.05 . This suggested only minor improvements in the correlation between the TRCs and the proteome. Use of TRC ${ }^{\mathrm{Max}}$, on the other hand, resulted in larger improvements of correlation. TRC ${ }^{\mathrm{Max}}$ revealed $45 \%$ (18 of 40 ) of improved cases, with changes between $0.05<r<0.886$. Also the frequency distribution of Pearson correlation coefficients (Figure 9) showed an increase in frequencies for the coeffients 0.3 and 0.8 , which might indicate a shift towards positive 1 for $\mathrm{TRC}^{\mathrm{Max}}$, which was not observed for TRC ${ }^{\text {Mean }}$. Taken together, this pilot study indicated the $\mathrm{MIT}^{\mathrm{Max}}$ score to be more biologically relevant in reflecting the biological mechanism of translation regulation compared to the $\mathrm{MIT}^{\mathrm{Mean}}$.

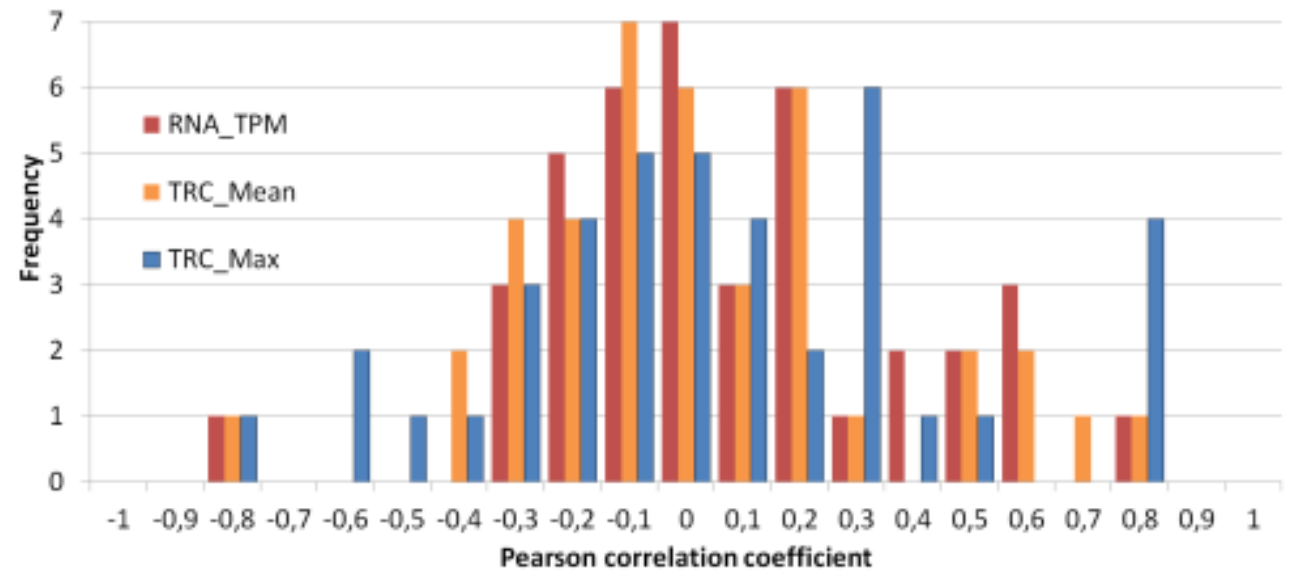

Figure 9: Frequency distribution of Pearson correlation coefficients. Obtained Pearson correlations were rounded to 1 decimal, after which the frequency of occurrence was counted for every correlation assessment (with protein abundance-TRC ${ }^{\mathrm{Max}}$ in blue, protein abundance-TRC ${ }^{\text {Mean }}$ in orange and protein abundance-unadjusted RNA in red). As expected, unadjusted RNA counts and TRC ${ }^{\text {Mean }}$ were very similar. $T_{R C}$ Max , however, showed an increase in frequency for correlation with a coefficient of 0.3 and 0.8 . Especially the increase at 0.8 indicates improved correlations 


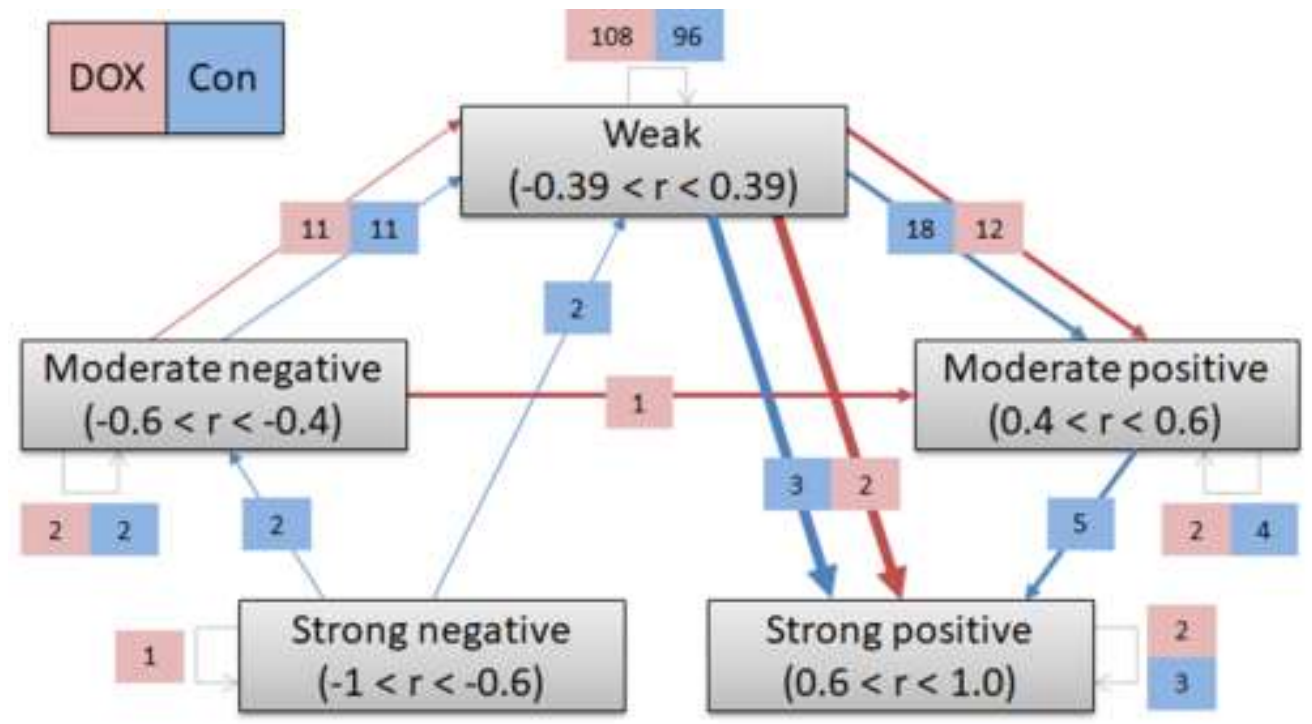

Figure 10: Correlation improvements by using TRC ${ }^{\mathrm{Max}}$ instead of unadjusted RNA read counts. Obtained correlation coefficients are grouped based on their values in weak, moderate or strong and in negative or positive correlations. The arrow depict the improvement of the correlation, with its origin depicting the correlation between protein and unadjusted RNA counts and pointing to the correlation found between protein and TRC ${ }^{\mathrm{Max}}$ values. The numbers placed on the arrows depict the amount of transcripts for which this improvement was observed. Furthermore, the thicknesses of the arrows indicate the importance for improving biological meaning, with thicker lines being more relevant. DOX exposed samples are depicted in red and control samples in blue

\section{Validation of MIT scores: Correlation of TRCMax with the proteome}

Now we have determined that the $\mathrm{MIT}^{\mathrm{Max}}$ score reflects the biological regulation of miRNA-induced translation regulation, we selected 287 transcripts with significant DOX-induced changes in MIT $^{\mathrm{Max}}$ scores and availability of proteomics data at every time point. Assessment of the correlation changes between protein-unadjusted RNA count and protein-TRC $^{\mathrm{Max}}$ (Supplementary Table II), revealed improvements of correlation for 146 transcripts (= 50.9\%) in control samples and 143 transcripts (= 49.8\%) in DOX-exposed samples. To obtain a more detailed overview, we categorized the obtained correlations in 5 groups based on their correlation coefficient, where after the improved correlations were depicted based on changes between these groups (Figure 10). We deemed some changes to be more biologically relevant (right side of Figure 10) than others (left side of Figure 10). For instance, a change from a weak correlation to a moderate or strong positive correlation clearly reflected an 
improvement of biological relevance, therefore these changes were represented by a thicker arrow. The fact that changes between groups were found more often for these relevant changes indicated the importance for assessment of post-transcriptional mechanisms.

\section{Is DOX able to induce changes in miRNA-circRNA regulation?}

To assess whether DOX was able to induce changes within miRNA-circRNA regulatory mechanisms, we selected transcripts of which $\mathrm{MIT}^{\mathrm{Max}}$ scores and proteomics data was available for every sample and at every time point. Overall, 480 transcripts were selected, of which 287 (60\%) displayed a significantly changed MIT ${ }^{\mathrm{Max}}$ score (students T-test $p<0.05)$. Of these significantly changed transcripts, $63 \%(n=181)$ displayed an increased MIT ${ }^{\mathrm{Max}}$ score. This suggested that DOX was capable of strongly decreasing RNA translation due to miRNA interference.

Interestingly, observed correlation improvements through the use of TRC ${ }^{\mathrm{Max}}$ were not transcript-specific. An improvement of correlation for control samples, as we already observed for APOBEC2 (Figure 8), did not imply an improvement for these transcripts in DOX-exposed samples (Figure $11 \mathrm{~A}-\mathrm{C}$ ) or the other way around (Figure 11D-F). This indicated that our scoring system used in this pilot study may be used to identify specific DOX-induced changes which may play a role in DOX-induced cardiotoxicity. 


\section{Discussion}

In order to quantify interactions between mRNA, miRNA and circRNA genome-wide, we designed a scoring system to estimate the fraction of miRNAs which are not captured by circRNAs ("free" miRNAs) and are therefore able to inhibit mRNAs translation into protein. This fraction constitutes the MIT score (miRNA inhibition of translation) and is calculated for every miRNA which is predicted to interact with a single target transcript. In order to interpret the multitude of obtained MIT scores per

A)

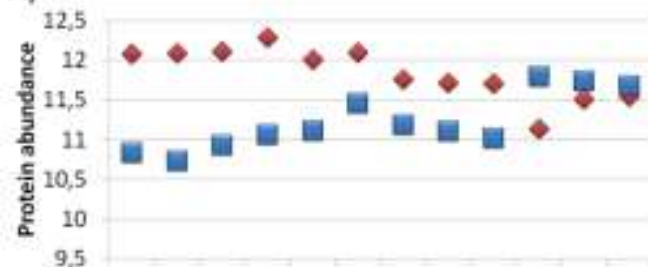

$e^{2} e^{2} e^{3} e^{2} e^{2} e^{3} e^{2} e^{2} e^{3} e^{2} e^{2} e^{3}$

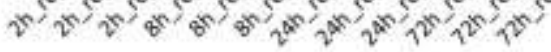

B)

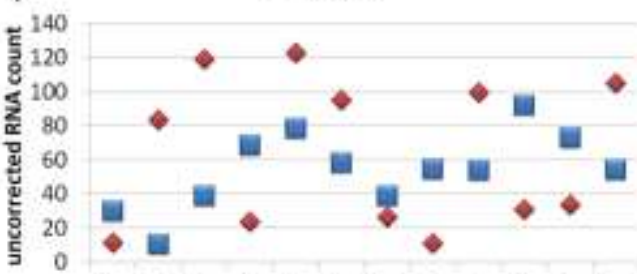

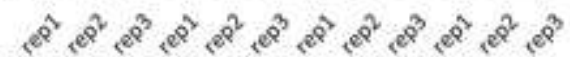

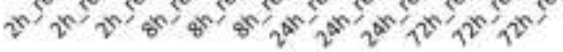

C)

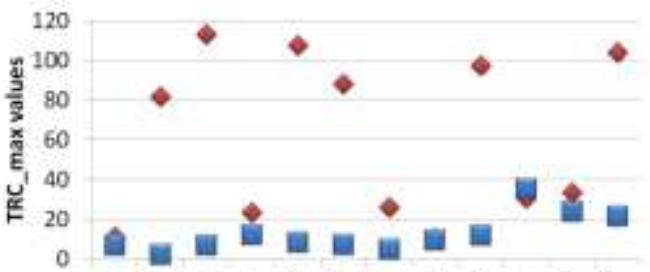

$e^{2} a^{2} a^{3} a^{3} a^{2} a^{3} a^{2} a^{2} a^{3} \cdot a^{2} e^{2} a^{3}$ vंv'
D)

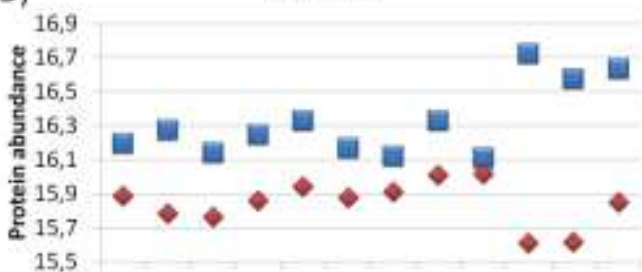

$a^{2} e^{2} e^{3} a^{2} e^{2} a^{3} a^{2} a^{2} a^{3} e^{2} e^{2} e^{3}$

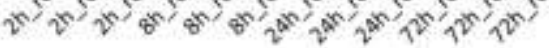

E)

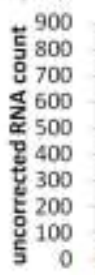

ENSG00000203879

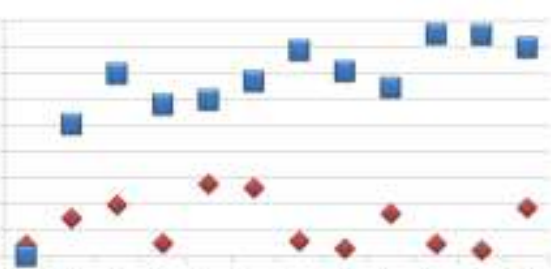

$a^{2} a^{2} a^{3} a^{2} a^{2} a^{3} a^{2} a^{2} a^{3} a^{2} a^{2} a^{3}$

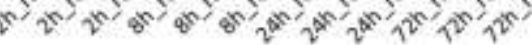

F)

ENSG00000203879

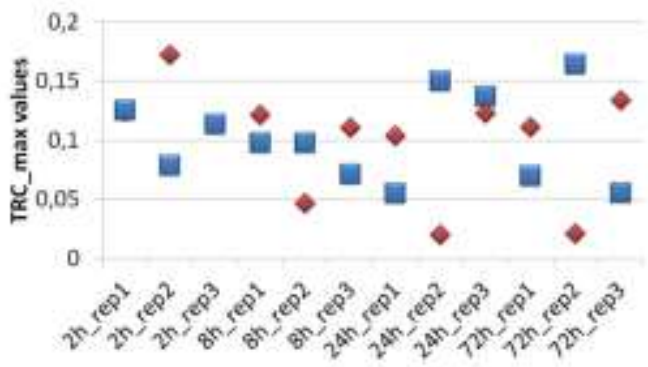

Figure 11: Correlation analysis of transcriptome and proteome. DOX exposed samples are depicted in red and control samples in blue. Every column contains values for a single protein-transcript set. A-D) protein abundance. B-E) mRNA expression. C-F) TRC ${ }^{\text {Max }}$ values 
transcript, we evaluate the applicability of the $\mathrm{MIT}^{\text {Mean }}$ and $\mathrm{MIT}^{\mathrm{Max}}$ scores to investigate which parameter was best suited to disclose relevant biological processes within the cell.

We used a data set reflecting DOX-induced changes in a human in vitro cardiac model at a therapeutic dose able to induce cardiotoxic side-effects. We obtained different conclusions when assessing significant changes in $\mathrm{MIT}^{\mathrm{Mean}}$ or $\mathrm{MIT}^{\mathrm{Max}}$ scores. While the MIT ${ }^{\text {Mean }}$ suggested that DOX decreased the inhibition capability of miRNAs for $60 \%$ of the 9803 analysed transcripts, the $\mathrm{MIT}^{\mathrm{Max}}$ indicated significant changes for $30 \%$ of transcripts, of which $<10 \%$ were showed decreased inhibition capability. Still, both measures confirm our hypothesis of DOX being able to change miRNA-circRNA regulation.

To assess whether the $\mathrm{MIT}^{\mathrm{Mean}}$ or the $\mathrm{MIT}^{\mathrm{Max}}$ best reflect the biological mechanism of translation regulation, we validated these scores using proteomics data for control samples using 40 selected protein-transcript sets. Because the MIT scores represented the fraction of miRNAs which can inhibit translation, we were able to use the inversed value to calculate the translatable read count (TRC) of transcripts. Through Pearson correlation analysis between the proteome and TRCs obtained using MIT $^{\text {Mean }}$ (TRC ${ }^{\text {Mean }}$ ) or $\mathrm{MIT}^{\mathrm{Max}}\left(\mathrm{TRC}^{\mathrm{Max}}\right)$, we were able to identify improvements of correlation which indicated the biologically relevance of our scoring system. While both TRC ${ }^{\text {Mean }}$ and $\mathrm{TRC}^{\mathrm{Max}}$ resulted in improved correlation with the proteome compared to unadjusted RNA counts ( $47.5 \%$ and $45 \%$ of analysed protein-transcript sets resp.), the improvements gained from TRC ${ }^{\text {Mean }}$ were minor $(<0.05)$. Using TRC ${ }^{\text {Max }}$ values, on the other hand, correlations not only improved more $(0.05<r<0.886)$, but when plotting the data, also the pattern was visually similar to the protein abundance (Figure 8\&9). This indicated a biologically meaningful improvement and suggested that the MIT ${ }^{\mathrm{Max}}$ scores better reflected the biological mechanisms of translation inhibition compared to the MIT ${ }^{\text {Mean }}$ scores. 
We also assessed differences in correlation improvements between control and DOX-exposed. Out of the 287 included protein-transcript sets, the use of TRC ${ }^{\mathrm{Max}}$ resulted in approximately $50 \%$ improved correlations in both conditions. In DOX and control samples different transcripts showed an improvement in correlation, which indicated the ability of the scoring system to identify specific DOX-induced changes of the circRNA-miRNA regulatory system.

To assess whether DOX was able to induce changes within miRNA-circRNA regulatory mechanisms, we selected 480 transcripts with for which $\mathrm{MIT}^{\mathrm{Max}}$ scores and proteomics data was available for every sample at every time point. $60 \%$ of these transcripts revealed a significant change in $\mathrm{MIT}^{\mathrm{Max}}$, of which the majority $(63 \%, \mathrm{n}=181)$ showed an increased miRNA-induced inhibition of translation. This suggests that DOX is capable of strongly decreasing RNA translation due to miRNA interference.

The scoring system described in this pilot study might benefit from expansion and some optimization for optimal use in the future. Currently, our scoring system covers interactions between mRNA, miRNAs and circRNAs. It would be most optimal to follow a system biology approach and incorporate all known post-transcriptional mechanisms in the scoring system. While this was not yet feasible, step by step expansion can be considered. For example, currently gene expression values were used for obtaining the amount of translatable mRNAs. However, through alternative splicing, non-coding transcripts may be expressed. Therefore, analysis of transcript isoforms to only include expression of protein coding transcripts may further increase the correlation between the transcriptome and the proteome. Furthermore, circRNAs have additional posttranscriptional effects through acting as RNA-binding protein (RBP) sponges which could greatly influence splicing, stability, cellular localization and translation of mRNAs. Re et al. ${ }^{[50]}$ already showed an improved accuracy of predicted protein abundances by assessing RBP-mRNA regulatory interactions. Incorporation of mRNA-RBP-circRNA interactions may therefore greatly improve our algorithm. Additionally, optimization of the current scoring system may be considered. For identification of circRNAs, we use the common protocol of Memczak et al. ${ }^{[39]}$. To drastically reduce the amount of false positive results, we only considered circRNAs which were independently predicted by 
both $\mathrm{R} 1$ and $\mathrm{R} 2$ reads $^{[51]}$. Thereafter, circRNA expression was calculated by remapping the sequencing reads to the sequences of the predicted circRNAs, meaning that also fragments resulting from linear RNAs are counted and expression of circRNAs are artificially increased.

The algorithm will probably benefit from using a newly introduced circRNA prediction approach. Because combining prediction protocols has been suggested to result in highly increased accuracy, a combination of the more recent CIRCexplorer2 ${ }^{\text {[52] }}$ and $\mathrm{CIRI} 2^{[53]}$ tools could be considered ${ }^{[51]}$. Furthermore, while our research identified the $\mathrm{MIT}^{\mathrm{Max}}$ score as being more accurate than the $\mathrm{MIT}^{\mathrm{Mean}}$ score, a possible innovation could be to adapt the scoring system to not calculate the effect per interaction miRNA, but to reflect the combined effects of all miRNAs that are able to influence a specific target transcript. However, in order to define a parameter resulting in realistic TRC values, actual measurements of the amount of translated mRNAs are necessary, which could be gained through a polysome assay ${ }^{[54]}$. Finally, also advances within Artificial Intelligence such as Machine Learning could be considered to improve the assessment of changed miRNA-circRNA regulation. Therefore, a bioinformatics approach to train, validate and test the scoring system could be used to gain a higher precision and understanding of the complex relationship between mRNA, miRNA and circRNA.

Overall, we showed that the estimation of translatable mRNA read counts using the $\mathrm{MIT}^{\mathrm{Max}}$ score correlated better with the proteome than unadjusted RNA counts do, indicating that assessment of post-transcriptional mechanisms is important for a deeper understanding of biological and toxicological processes when analysing transcriptomics data. Furthermore, our pilot study has shown the ability of our scoring system to identify changes within the miRNA-circRNA regulatory system. Using this scoring system we identified the capability of DOX to increase translation inhibition, which may play a role in DOX-induced cardiotoxicity at therapeutic doses. 
• • C Chapter 5: MicroRNA \& circular RNA interactions

Supplementary materials

Supplementary table 1: Correlation of transcriptome with proteome, 40 protein-transcript sets

ftp://web.tgx.unimaas.nl/mverheijen/Chapter5/Supplementary_table1.xlsx

Supplementary table 2: Correlation of transcriptome with proteome, 287 proteintranscript sets

$\mathrm{ftp}: / /$ web.tgx.unimaas.nl/mverheijen/Chapter5/Supplementary_table2.xlsx 


\section{References}

1 Chen, Z. J. et al. Fresh versus Frozen Embryos for Infertility in the Polycystic Ovary Syndrome. New Engl J Med 375, 523-533, doi:10.1056/NEJMoa1513873 (2016).

2 Payne, S. H. The utility of protein and mRNA correlation. Trends Biochem Sci 40, 1-3, doi:10.1016/j.tibs.2014.10.010 (2015).

3 Ghazalpour, A. et al. Comparative Analysis of Proteome and Transcriptome Variation in Mouse. Plos Genet 7, doi:ARTN e1001393 10.1371/journal.pgen.1001393 (2011).

4 Gry, M. et al. Correlations between RNA and protein expression profiles in 23 human cell lines. BMC genomics 10, doi:Artn 365 10.1186/1471-2164-10-365 (2009).

5 Edfors, F. et al. Gene-specific correlation of RNA and protein levels in human cells and tissues. Mol Syst Biol 12, 883, doi:10.15252/msb.20167144 (2016).

6 Koussounadis, A., Langdon, S. P., Um, I. H., Harrison, D. J. \& Smith, V. A. Relationship between differentially expressed mRNA and mRNA-protein correlations in a xenograft model system. Sci Rep-Uk 5, doi:ARTN 10775 10.1038/srep10775 (2015).

7 Vogel, C. \& Marcotte, E. M. Insights into the regulation of protein abundance from proteomic and transcriptomic analyses. Nature Reviews Genetics 13, 227-232, doi:10.1038/nrg3185 (2012).

8 Gygi, S. P., Rochon, Y., Franza, B. R. \& Aebersold, R. Correlation between protein and mRNA abundance in yeast. Mol Cell Biol 19, 1720-1730 (1999).

9 Anderson, L. \& Seilhamer, J. A comparison of selected mRNA and protein abundances in human liver. Electrophoresis 18, 533-537, doi:DOI 10.1002/elps.1150180333 (1997).

10 Maier, T., Guell, M. \& Serrano, L. Correlation of mRNA and protein in complex biological samples. Febs Lett 583, 3966-3973, doi:10.1016/j.febslet.2009.10.036 (2009).

11 Maier, T. et al. Quantification of mRNA and protein and integration with protein turnover in a bacterium. Molecular Systems Biology 7, doi:ARTN 511 10.1038/msb.2011.38 (2011).

12 Lundberg, E. et al. Defining the transcriptome and proteome in three functionally different human cell lines. Molecular Systems Biology 6, doi:ARTN 450 10.1038/msb.2010.106 (2010).

13 Schwanhausser, B. et al. Corrigendum: Global quantification of mammalian gene expression control. Nature 495, 126-127, doi:10.1038/nature11848 (2013).

14 Lawless, C. et al. Direct and Absolute Quantification of over 1800 Yeast Proteins via Selected Reaction Monitoring. Mol Cell Proteomics 15, 1309-1322, doi:10.1074/mcp.M115.054288 (2016).

15 Nagaraj, N. et al. Deep proteome and transcriptome mapping of a human cancer cell line. Molecular Systems Biology 7, doi:ARTN 548 10.1038/msb.2011.81 (2011).

16 Zhao, B. X. S., Roundtree, I. A. \& He, C. Post-transcriptional gene regulation by mRNA modifications. Nat Rev Mol Cell Bio 18, 31-42, doi:10.1038/nrm.2016.132 (2017).

17 da Costa, P. J., Menezes, J. \& Romao, L. The role of alternative splicing coupled to nonsense-mediated mRNA decay in human disease. Int J Biochem Cell B 91, 168-175, doi:10.1016/j.biocel.2017.07.013 (2017).

18 Duchaine, T. F. \& Fabian, M. R. Mechanistic Insights into MicroRNA-Mediated Gene Silencing. Csh Perspect Biol, a032771 (2018).

19 Chen, X. C. et al. Long noncoding RNA: multiple players in gene expression. Bmb Rep 51, 280-289, doi:10.5483/BMBRep.2018.51.6.025 (2018).

20 Ingolia, N. T., Hussmann, J. A. \& Weissman, J. S. Ribosome Profiling: Global Views of Translation. Cold Spring Harb Perspect Biol, doi:10.1101/cshperspect.a032698 (2018).

21 Heck, A. M. \& Wilusz, J. The Interplay between the RNA Decay and Translation Machinery in Eukaryotes. Csh Perspect Biol 10, doi:ARTN a032839 10.1101/cshperspect.a032839 (2018).

22 Verheijen, M., Krauskopf, J., Kleinjans, J. C., de Kok, T. M. \& Caiment, F. Development and regulatory application of microRNA biomarkers. Biomark Med 9, 1137-1151, doi:10.2217/bmm.15.50 (2015).

23 Aonuma, T., Bayoumi, A. S., Tang, Y. \& Kim, I.-m. A circular RNA regulator quaking: a novel gold mine to be unfolded in doxorubicin-mediated cardiotoxicity. Non-coding RNA Investigation (2018).

24 Oliveto, S., Mancino, M., Manfrini, N. \& Biffo, S. Role of microRNAs in translation regulation and cancer. World J Biol Chem 8, 45-56, doi:10.4331/wjbc.v8.i1.45 (2017).

25 Tang, L. et al. microRNA inhibitors: Natural and artificial sequestration of microRNA. Cancer Lett 407, 139-147, doi:10.1016/j.canlet.2017.05.025 (2017).

26 Liu, J., Liu, T., Wang, X. \& He, A. Circles reshaping the RNA world: from waste to treasure. Mol Cancer 16, 58, doi:10.1186/s12943-017-0630-y (2017). 
27 Qu, S. B. et al. Circular RNA: A new star of noncoding RNAs. Cancer Lett 365, 141-148, doi:10.1016/j.canlet.2015.06.003 (2015).

28 Memczak, S. et al. Circular RNAs are a large class of animal RNAs with regulatory potency. Nature 495, 333-338, doi:10.1038/nature11928 (2013).

29 Maillet, A. et al. Modeling Doxorubicin-Induced Cardiotoxicity in Human Pluripotent Stem Cell DerivedCardiomyocytes. Sci Rep 6, 25333, doi:10.1038/srep25333 (2016).

30 Cappetta, D., Urbanek, K., Rossi, F. \& De Angelis, A. Anthracycline cardiotoxicity: new actors on the stage. Transl Cancer Res 7, S580-S583, doi:10.21037/tcr.2018.04.24 (2018).

31 McGowan, J. V. et al. Anthracycline Chemotherapy and Cardiotoxicity. Cardiovasc Drug Ther 31, 63-75, doi:10.1007/s10557-016-6711-0 (2017).

32 Bernstein, D. Anthracycline Cardiotoxicity Worrisome Enough to Have You Quaking? Circ Res 122, 188190, doi:10.1161/Circresaha.117.312395 (2018).

33 Chatterjee, K., Zhang, J. Q., Honbo, N. \& Karliner, J. S. Doxorubicin Cardiomyopathy. Cardiology 115, 155162, doi:10.1159/000265166 (2010).

34 Kuepfer, L. et al. in Intracellular Delivery III: Market Entry Barriers of Nanomedicines (eds Aleš Prokop \& Volkmar Weissig) 363-374 (Springer International Publishing, 2016).

35 Bolger, A. M., Lohse, M. \& Usadel, B. Trimmomatic: a flexible trimmer for Illumina sequence data. Bioinformatics 30, 2114-2120, doi:10.1093/bioinformatics/btu170 (2014).

36 Andrews, S. FastQC: a quality control tool for high throughput sequence data. http://www.bioinformatics.babraham.ac.uk/projects/fastqc (2010).

37 Li, B. \& Dewey, C. N. RSEM: accurate transcript quantification from RNA-Seq data with or without a reference genome. Bmc Bioinformatics 12, doi:Artn 323 10.1186/1471-2105-12-323 (2011).

38 Langmead, B. \& Salzberg, S. L. Fast gapped-read alignment with Bowtie 2. Nat Methods 9, 357-U354, doi:10.1038/Nmeth.1923 (2012).

39 Memczak, S. et al. Circular RNAs are a large class of animal RNAs with regulatory potency. Nature 495 , 333 (2013).

40 Caiment, F., Gaj, S., Claessen, S. \& Kleinjans, J. High-throughput data integration of RNA-miRNA-circRNA reveals novel insights into mechanisms of benzo [a] pyrene-induced carcinogenicity. Nucleic Acids Res 43, 2525-2534 (2015).

41 Durinck, S. et al. BioMart and Bioconductor: a powerful link between biological databases and microarray data analysis. Bioinformatics 21, 3439-3440 (2005).

42 Caiment, F., Gaj, S., Claessen, S. \& Kleinjans, J. High-throughput data integration of RNA-miRNA-circRNA reveals novel insights into mechanisms of benzo[a]pyrene-induced carcinogenicity. Nucleic Acids Res 43, 2525-2534, doi:10.1093/nar/gkv115 (2015).

43 Prufer, K. et al. PatMaN: rapid alignment of short sequences to large databases. Bioinformatics 24, 15301531, doi:10.1093/bioinformatics/btn223 (2008).

44 Enright, A. J. et al. MicroRNA targets in Drosophila. Genome Biol 5, R1 (2003).

45 Silva, J. C., Gorenstein, M. V., Li, G. Z., Vissers, J. P. C. \& Geromanos, S. J. Absolute quantification of proteins by LCMSE - A virtue of parallel MS acquisition. Mol Cell Proteomics 5, 144-156, doi:10.1074/mcp.M500230-MCP200 (2006).

46 Haque, S. \& Harries, L. W. Circular RNAs (circRNAs) in health and disease. Genes 8, 353 (2017).

47 Cortes-Lopez, M. \& Miura, P. Emerging Functions of Circular RNAs. Yale J Biol Med 89, 527-537 (2016).

48 Panda, A. C., Grammatikakis, I., Munk, R., Gorospe, M. \& Abdelmohsen, K. Emerging roles and context of circular RNAs. Wiley Interdiscip Rev RNA 8, doi:10.1002/wrna.1386 (2017).

$49 \mathrm{Li}, \mathrm{Z}$. et al. Exon-intron circular RNAs regulate transcription in the nucleus. Nat Struct Mol Biol 22, $256-$ 264, doi:10.1038/nsmb.2959 (2015).

$50 \mathrm{Re}, \mathrm{A} ., \mathrm{Waldron}, \mathrm{L}$. \& Quattrone, A. Control of Gene Expression by RNA Binding Protein Action on Alternative Translation Initiation Sites. Plos Comput Biol 12, doi:ARTN e1005198 10.1371/journal.pcbi.1005198 (2016).

51 Hansen, T. B. Improved circRNA Identification by Combining Prediction Algorithms. Front Cell Dev Biol 6, 20, doi:10.3389/fcell.2018.00020 (2018).

52 Zhang, X. O. et al. Diverse alternative back-splicing and alternative splicing landscape of circular RNAs. Genome Res 26, 1277-1287, doi:10.1101/gr.202895.115 (2016).

53 Gao, Y., Zhang, J. \& Zhao, F. Circular RNA identification based on multiple seed matching. Briefings in bioinformatics, doi:10.1093/bib/bbx014 (2017).

54 Smit, E., Caiment, F., Piepers, J., Kleinjans, J. C. \& van den Beucken, T. Translational regulation is a key determinant of the cellular response to benzo [a] pyrene. Toxicology letters (2018). 
• • C Chapter 5: MicroRNA \& circular RNA interactions . • • 

Chapter 6

Summary and General Discussion 
Current drug development and safety testing procedures are time consuming, expensive and not able to detect all human drug-induced toxicity risks, with occasional severe adverse drug reactions as a consequence. The adverse drug reactions also rank high as cause for disease or death ${ }^{[1]}$. In search for methods to improve in vitro toxicological drug safety assessments, we adopted an experimental design better reflecting the human in vivo conditions and investigated possibilities to improve toxicogenomics data analysis. Here, we predominantly focused on post-transcriptional mechanisms.

As an example toxicant, we mainly used doxorubicin (DOX), but to some extent also epirubicin (EPI) and idarubicin (IDA) were studied in vitro in the human cardiac 3D microtissue model. These chemotherapeutic agents belong to the group of anthracyclines (ANTs) and are known to dose-dependently induce cardiotoxicity ${ }^{[2,3]}$. The occurrence of cardiotoxicity is difficult to predict, because it can manifest at any stage during the treatment (reversible acute cardiotoxicity) or even months or years after the treatment has ended (irreversible delayed chronic cardiotoxicity) while the involved molecular mechanisms are only partially understood ${ }^{[2-6]}$.

\section{Experimental design}

Within toxicology, the experimental design typical includes the use of a solvent like Dimethyl sulfoxide (DMSO) in order make a stable stock solution of the investigated compound. However, in the 1960s DMSO was researched for medical use due to its biological effects. During the clinical trials, high doses of DMSO induced extreme toxicities, especially to the eyes, though. Because the effects of DMSO differ depending on dose and route of administration, the FDA classified DMSO in the safest category with low toxic potential at levels normally accepted in pharmaceuticals ${ }^{[7]}$. This made the wide use of DMSO possible. 
Though it often assumed that effects of DMSO are negligible ${ }^{[8-10]}$, we evaluated the impact of $0.1 \%$ DMSO exposure using sensitive high-throughput techniques (Chapter 2). We did this for the cardiac microtissue model relevant for this thesis, but also for the hepatic microtissue model used by other partners of the HeCaToS project.

DMSO effects on cellular processes were analyzed using full transcriptome data supplemented with proteomics data. More than 2000 differentially expressed genes (DEGs, FDR <0.05) and 650 differentially expressed proteins (DEPs, FDR <0.05) were detected in both tissue types, of which more than half were downregulated. Pathway analysis indicated that similar biological processes were affected in both cell types, indicating consistent cross-organ actions of DMSO.

Observed DMSO-induced changes in cellular processes included changes in mitochondrial pathways linked to ROS production and cellular ATP generation. Functional measurements of microtissue ATP content for the hepatic model revealed a small initial decrease ( $36 \%$ decrease in the first $72 \mathrm{~h}$ ), after which ATP content slowly recovered to baseline level. However, a steep decrease over time $(87 \%$ decrease after 2 weeks of exposure) was observed for cardiac microtissues. This decrease in ATP production may impact toxicological research because changes in the amount of free energy in the cell influences the capability of the cell to deal with induced stresses, thereby potentially leading to erroneous interpretation of results from in vitro assays. The DMSO-induced ATP decrease is of even more relevance for the field of assisted reproductive technology, in which DMSO is used for cryopreservation of oocytes and embryos for IVF treatments. Here the ATP content is used as a predictor of embryo viability. Decreased ATP levels, especially in the cleavage-stage, can induce downstream effects that may disrupt cellular function, implantation ability and fetal development ${ }^{[11,12]}$.

While observed tissue-specific differences may be due to tissue type, they may also result from a fundamental difference in the cell model, because the cardiac model contains iPSC-derived human cardiomyocytes that are still maturing, while the hepatic 
model contains mature human hepatocytes. Expanding our research to the epigenetic landscape also revealed tissue-specific effects of DMSO. Analysis of miRNAs indicated a complete deregulation of cardiac miRNA biogenesis and miRNA content. Though changes in hepatic miRNA biogenesis were minimal, still $18 \%$ of detected miRNAs were significantly changed by DMSO exposure.

Furthermore, we showed, for the first time, the influence of DMSO on the DNA methylation patterns of human cells in vitro. Whole genome methylation profiling by MeDIP-seq revealed 66,178 differentially methylated regions (DMRs; q-value $<0.05$ ) in cardiac microtissues with $71 \%$ hypermethylated, while in hepatic microtissues, no DMRs passed correction for multiple testing. While repetitive sequences appeared preferentially affected, findings also suggested a global deregulation of DNA methylation mechanisms leading to genome-wide changes. It is uncertain if exposed cells can recover from temporary DMSO exposures. While methylation changes have an adaptive nature, they may also be persistent and even heritable by the offspring to induce transgenerational effects ${ }^{[13-17]}$. This may pose a threat, especially for cryopreserved oocytes and embryos for IVF treatment which are exposed to high concentrations of DMSO during the periconceptional period (before conception until early pregnancy), when epigenetic reprogramming is taking place.

Overall, the extreme changes in microRNAs and alterations in the epigenetic landscape indicated that DMSO is not inert. Therefore, use of DMSO should be avoided where possible. However, for the time being, DMSO is indispensable within biotechnological applications, including toxicological research. To reduce the impact of DMSO, concentrations should be kept as low as possible.

A decrease in DMSO concentration was incorporated within the innovative in vitro experimental design which we validated in Chapter 3. Next to using the human cardiac microtissue model, which better resembles the human heart in vivo due to its 3D spheroid structure and the co-culturing of both cardiomyocytes and fibroblasts, we also included physiologically based pharmacokinetic (PBPK) modelling in order to 
achieve a repetitive dosing profile which resembled the in vivo drug concentrations in the human heart. The highest exposure was $0.06 \%$ DMSO for 2 hours per day, continuing with $0.006 \%$ for 6 hours and ending with $0.0025 \%$ for 16 hours.

This experimental design was validated by assessing whether our model was able to retrieve the known mechanisms of DOX through pathway analysis and in depth analysis of mitochondrial dysfunction. While DOX mechanisms of action are not fully understood, it is generally accepted that the main mode of action is related to killing dividing cells. DOX has been found to 1 ) intercalate into DNA, 2) target DNA topoisomerases, and 3 ) generate reactive oxygen species $(\mathrm{ROS})^{[18-20]}$. The first two processes inhibit unwinding of DNA, DNA replication, RNA transcription and protein biosynthesis. As a result, proliferation of dividing cells is also inhibited ${ }^{[21,22]}$. This is thought to be the efficacy of the anti-cancer effects of DOX, while ROS generation is mainly ascribed to toxic effects ${ }^{[23]}$. The induced oxidative stress may damage cells and cause cell death ${ }^{[19,24]}$. Other detrimental actions of DOX can be related to death of noncancer cells or to decreased cardiomyocyte functioning, which may partly result from mitochondrial dysfunction causing an imbalance in cellular energetics ${ }^{[25]}$. The detrimental actions of DOX, known to occur in vivo, can thus be summarized into 3 toxicity processes: cardiomyocyte dysfunction, mitochondrial dysfunction and cell death. We were able to successfully detect biological changes in these three processes in our in vitro model by applying next-generation total RNA-sequencing to ribodepleted RNA samples.

Pathway analysis of differentially expressed genes (FDR $<0.05)$ identified toxicity of DOX by gene expression changes in the pathways of "striated muscle contraction" (= cardiac dysfunction), "TCA cycle \& respiratory electron transport" (= mitochondrial dysfunction) and "cellular senescence" (= cell death). A central link between the toxicity pathways was the "formation of ATP by chemiosmotic coupling". Mitochondrial gene expression changes correlated with ATP measurements, in which the therapeutic dose displayed a relatively stable ATP content, while ATP content during toxic exposure decreased drastically over time. A more detailed analysis of mitochondrial dysfunction revealed gene expression changes in the process of ROS 
detoxification, which confirms DOX-induced ROS generation in our in vitro model. Furthermore, effects of DOX on the electron transport chain (ETC) and ATP production was observed, with upregulations across time for the therapeutic exposure and a majority of downregulations during the second half of toxic exposures.

Many studies on DOX effects have been performed with high doses in order to observe toxic effects. However, our in vitro model clearly demonstrated a dosedependent effect of DOX exposure, highlighting the importance of applying physiologically relevant dosing profiles during toxicological research. Exposure to the toxic dosing profile reflected processes of acute cardiotoxicity, as observed though a decrease in ATP content and an increase in cell death. On the other hand, therapeutic doses reflected processes underlying the phenotype of delayed chronic cardiac toxicity. The increase in gene expression of mitochondrial and ETC genes was necessary in order to maintain a stable cellular ATP content, indicating an adaptation response. Because genes involved in striated muscle contraction were already showing decreased expression levels, this adaptation response may not be sufficient for maintaining cardiac function. Cumulative toxicity may therefore surpasses the point to which the organ can adapt, thereafter resulting in a delayed phenotype of heart failure, as is observed in patients with chronic cardiotoxicity after DOX treatment ${ }^{[25,26]}$. These same dose-dependent effects were also observed for the other ANTs (EPI and IDA) analysed in Chapter 4.

\section{Advanced transcriptomics analysis}

In Chapter 4, we went beyond conventional gene-expression analysis by investigation of ANT-induced changes in alternative splicing. Through alternative splicing, a single gene encodes multiple variants of RNA transcripts with distinct functions ${ }^{[27]}$. This greatly increases the diversity of the transcriptome ${ }^{[28,29]}$, which can be measured using RNA-sequencing technology. It is estimated that $92-94 \%$ of human genes are subjected to alternative splicing ${ }^{[27]}$. During gene expression analysis, the sum of these transcripts is assessed. However, due to differences in biological function of alternatively spliced transcripts, not only changes in gene expression, but also changes 
in ratios of expressed transcripts (differential transcript usage; DTU) can result in a distinct phenotype ${ }^{[29,30]}$. Therefore it is important to make use of technological advances of recent years to analyze DTU for elucidating toxicological mechanisms.

ANT-induced changes in alternative splicing were investigated for sarcomeric genes. The sarcomere is the cellular compartment facilitating contraction and is therefore highly important for heart function and cardiotoxic effects. The most interesting ANTinduced changes were observed for the titin gene (TTN) which is essential for maintaining the structure of the sarcomere because it forms the base for the assembly of the contractile machinery. During relaxation, its protein product unfolds and refolds during contraction. Furthermore, the in vitro observed ANT-induced changes could also be detected in human biopsies of heart failure patients with or without prior ANT therapy, confirming the clinical relevance of these observed effects. While the expression of the titin gene (TTN) differed between the ANTs, ranging from no significant changes (IDA) to downregulated at every time point (EPI), DTU analysis identified the most significant change in transcript usage for the TTN-206 transcript in all three ANTs (FDR: $6.96 \cdot 10^{-32}, 2.04 \cdot 10^{-31} \& 1.38057 \cdot 10^{-39}$ for DOX, EPI \& IDA, resp.). The decrease in TTN-206 transcript usage was correlated in vivo with increased left ventricular (LV) mass index $(p=0.002)$, decreased LVEF $(p=0.018)$ and was the only transcript close to significant $(\mathrm{P}=0.07)$ when comparing dilated vs undilated morphologies. The switch form TTN-206 (encoding H7C1P9, 962aa) to TTN-212 (encoding Q8WZ42, 26.926aa) changes the expression of a truncated titin protein variant. These truncated variants have the ability to impact the structural organization of sarcomeres and to disturb regulation of sarcomeric passive force generation and reduce the efficiency of contraction ${ }^{[31-33]}$.

To summarize, while changes in overall TTN gene expression were unable to identify interesting ANT-induced effects, analysis of DTUs identified the decrease of TTN-206 which can be a highly promising as a biomarker for early detection of ANT-induced cardiotoxicity because it correlated with increased LV mass index, decreased LVEF and 
can possibly distinguish between dilated and unaffected morphologies. This shows the added value of biological information to be gained from DTU analysis as compared to standard gene expression analysis.

Next to analysis of alternative splicing, other post-transcriptional mechanisms can also be investigated using RNA sequencing technologies. In Chapter 5, we focused on the incorporation of microRNA (miRNA) and circular RNA (circRNA) regulatory actions in transcriptomics analysis. MicroRNAs, small non-coding RNAs of approximately 22 nucleotides ${ }^{[1,34]}$, can bind through complementary base pairing to regions within the 3'UTR of the mRNA (known as seed regions). This interaction can inhibit the translation of a mRNA. Furthermore, circRNAs, which are closed continuous loops without $5^{\prime}$ caps or $3^{\prime}$ tails which are generated through head-to-tail mRNA splicing ${ }^{[35,36]}$, can also bind miRNAs. Because these circRNAs are highly stable and resistant against degradation, the miRNAs are captured and their inhibitory effect on translation is prevented ${ }^{[37]}$.

Because regulatory mechanisms acting on translation are proposed to be the main cause for the notoriously poor correlation between the transcriptome and the proteome ${ }^{[38-40]}$, we postulate that the assessment of these post-transcriptional mechanisms is important. On the short term, this will increase our understanding of biological and toxicological processes. On the long term, we might be able to incorporate the obtained knowledge to design an in silico prediction system to estimate the amount of translatable mRNAs, thereby obtaining a highly detailed genome-wide measurement with a biological relevance similar to proteomics data which can be used for toxicological assessments. We therefore designed a scoring system for genome-wide estimation of post-transcriptional miRNA and circRNAs interactions on mRNA translation. This scoring system estimates the fraction of miRNAs which are able to inhibit mRNAs translation because they are not captured by circRNAs. We labelled the obtained score as the miRNA-induced inhibition of translation (MIT) score, of which the calculation was based on the expression values and predicted interactions of mRNA, miRNA and circRNA. 
Using the cardiac microtissues exposed to the therapeutic DOX dosing profile, we conducted a pilot study to validate our scoring system. Because a MIT score was obtained for every miRNA able to influence a target transcript, tens to hundreds of MIT scores were obtained per transcript. For interpretation of these MIT scores, we assessed the use of 1) MIT Mean, which reflected the average inhibition efficiency of all miRNAs influencing a transcript, and 2) MIT ${ }^{\mathrm{Max}}$, which reflected the maximum MIT score because we envisioned that this miRNA was responsible for the inhibitory effect while effects of other miRNAs might be negligible in comparison. To determine which of these parameters best disclosed relevant biological processes within the cell, we first estimated the amount of translatable read count (TRC) using the MIT ${ }^{\text {Mean }}$ and the $\mathrm{MIT}^{\mathrm{Max}}$. Thereafter, we assessed whether the correlation between the proteome and the TRCs was improved compared to the correlation of the proteome with RNA read counts. We found improvements of correlation for $47.5 \%$ of cases using TRC ${ }^{\text {Mean }}$ values and $45 \%$ of cases using TRC ${ }^{\mathrm{Max}}$ values. While the absolute improvement of TRC ${ }^{\text {Mean }}$ was very low $(\Delta r<0.05), T^{M} C^{\mathrm{Max}}$ indicated more relevant improvements of correlation $(0.05$ $<\Delta r<0.886)$. Furthermore, when plotting the protein-abundances and TRC ${ }^{\text {Max }}$ values, the obtained graphs were visually very similar, which was not the case for RNA read counts. This demonstrated the ability of the MIT ${ }^{\mathrm{Max}}$ and the $\mathrm{TRC}^{\mathrm{Max}}$ to reflect biologically relevant translation inhibition processes.

Using the scoring system, we identified significant DOX-induced changes in miRNAcircRNA regulation in $60 \%$ of investigated transcripts $(n=480)$. Because $63 \%$ of the significantly changes comprised an increase in $\mathrm{MIT}^{\mathrm{Max}}$ score, we concluded that DOX was capable of strongly decreasing RNA translation through changes in regulatory miRNA-circRNA processes. Overall, the ability of the scoring system to identify changes in miRNA-circRNA regulatory systems as confirmed by the proteomics data, highlights the importance for assessment of post-transcriptional mechanisms, not only in toxicogenomics research but in genomics research in general. 


\section{Conclusions and Future perspectives}

We hypothesized that the use of an experimental design better reflecting the human in vivo conditions combined with analysis of post-transcriptional mechanisms would aid the evolution of toxicogenomics research in order to improve drug safety assessments in the future. Using the ANTs as a case study, we indeed showed the possibilities of improving the experimental design to relate more closely to the in vivo situation and displayed the vast amount of information gained from studying posttranscriptional mechanisms.

In Chapter 5 we showed the importance for quantifying post-transcriptional effects of miRNA-circRNA regulation of translation through the use of a scoring system to identify biologically relevant toxicant-changes which may play a role in toxicity.

Because the scoring system is currently restricted to the estimation of miRNAs and circRNAs regulatory effects, expansion to include other post-transcriptional mechanisms (such as alternative splicing) may be considered. Furthermore, the current scoring system may benefit from optimization through the use of more recently developed tools for circRNA prediction approaches (e.g. CIRCexplorer $2^{[41]}$ or $\mathrm{CIRI}^{[42]}$ ) and possibly the use of an alternative parameter for optimal interpretation. While our pilot study indicated that the miRNA with the maximum MIT score was better suited for biological interpretation of translation inhibition, compared to the average of the obtained MIT scores, this means that effects of other miRNAs are neglected. Alternatively, the scoring system could be adapted to return the combined effects of all miRNAs affecting a target transcript. Another possibility would be to use a machine learning approach which trains, validates and tests the scoring system in order to improve the precision of the prediction. However, most important would be to validate the scoring system using a multitude of compounds for which the results can be validated using literature or laboratory experiments. 
In Chapter 4, analysis of DTU revealed highly significant changes for the titin gene, which were not detected using observed standard gene expression analysis. This clearly demonstrated the added value for analyzing RNA splicing effects after exposure to a toxicant.

Human patient biopsies were used to validate in vitro DTU observations in vivo. Because of the low number of biopsies available from ANT treated patients $(n=7)$ and the observed heterogeneity of these human biopsies, the research would clearly benefit from additional validation efforts using more patient samples. Recently we obtained a new batch of human cardiac biopsies which could be included in the analysis, resulting in a total of 15 biopsies available from ANT treated patients. Furthermore, because the biological function of many of transcript isoforms has not been determined, we could only speculate about the biological impact of changes observed for these transcripts. Loss-of-function assays (e.g. RNAi screening ${ }^{[43]}$ ) to elucidate the functions of transcript variants are necessary (for both coding and noncoding transcripts) in order to draw reliable biological conclusions. Finally, our cell model contains induced pluripotent stem cells (iPSC)-derived cardiomyocytes which are known to acquire genetic variation during reprogramming of the cells, implying that batches of microtissues were not identical between the different ANT-exposures. Therefore, observed DTU in vitro could also have been due to different single nucleotide polymorphisms (SNPs) existing between the batches. Therefore, for the presented study, we regarded findings as reliable when detected in both in vitro microtissues and in vivo biopsies. For future research on DTU using iPSC-derived cell models, a multitude of iPSC batches should be exposed to obtain results across a variety of genomic backgrounds and the iPSC batches should be identical between exposure studies in order to facilitate comparability. 
In Chapter 3, our in vitro model including 3D microtissues and PBPK-based exposure profiles revealed a clear difference in activated biological mechanisms between the therapeutic and the toxic ANT exposure profile. This clearly indicates that future toxicological research should be performed at doses to which the human population can be exposed in order to obtain relevant safety assessments.

It should be taken into account that here only the effect of DOX on heart microtissues was investigated. For developing a predictive model for repeated dose heart toxicity, future research should thus be performed using additional toxicants and other tissue types (e.g. liver microtissues). Furthermore, next to 3D microtissues, also other in vitro systems mimicking the in vivo environment have been developed (e.g. organoids) which could also be of interest for toxicological research. Similar to microtissues, organoids are in vitro 3D models gained from pluripotent stem cells (either embryonic or induced) or primary tissue ${ }^{[44]}$. Organoids are physiologically closer to in vivo then microtissues because they contain more cell types, are larger in size and are capable of self-renewal. Because of this self-renewal, they can also be cultured for extended time. These features would be beneficial for toxicity assessment. However, culturing techniques for organoids are much more complex compared to culturing of microtissues, which could hamper large scale toxicity assessments.

Chapter 2 revealed that, contradictory to general assumptions, DMSO is not an inert solvent. Even at the commonly used "low" concentration of $0.1 \%$, DMSO was able to induce extreme changes in the epigenetic landscape. With the development of more sensitive analysis techniques, the doses used for toxicological research are also decreasing. In Chapter 3 we highlighted the need for investigating relevant doses. For DOX, the highest used doses were $0.22 \mu \mathrm{M}$ and $0.65 \mu \mathrm{M}$ for the therapeutic and toxic ANT exposure respectively. Compared to these low doses, these extremely high concentration of $14.1 \mathrm{mM}$ DMSO (=0.1\%) could induce changes which may result in erroneous conclusions gained from in vitro assays, such as false negative drug toxicity conclusions. Which brings us to a final but very strong message: In the future, avoid using DMSO where possible! 
Overall, this thesis demonstrates the benefits of using advanced models \& methods for toxicogenomics research. We introduced an advanced experimental design better reflecting the human in vivo conditions through incorporation of a 3D cell culture model, exposure to physiologically relevant concentrations and a reduction of the DMSO concentration. Furthermore, we highlighted the added value of advanced toxicogenomics data analysis through assessment of post-transcriptional mechanisms such as alternative splicing and translational regulation by miRNAs and circRNAs. Application of this model and these methods could improve future in vitro toxicological drug safety assessments. 


\section{References}

1 Verheijen, M., Krauskopf, J., Kleinjans, J. C., de Kok, T. M. \& Caiment, F. Development and regulatory application of microRNA biomarkers. Biomark Med 9, 1137-1151, doi:10.2217/bmm.15.50 (2015). Bernstein, D. Anthracycline Cardiotoxicity Worrisome Enough to Have You Quaking? Circ Res 122, 188190, doi:10.1161/Circresaha.117.312395 (2018). McGowan, J. V. et al. Anthracycline Chemotherapy and Cardiotoxicity. Cardiovasc Drug Ther 31, 63-75, doi:10.1007/s10557-016-6711-0 (2017). Maillet, A. et al. Modeling Doxorubicin-Induced Cardiotoxicity in Human Pluripotent Stem Cell DerivedCardiomyocytes. Sci Rep 6, 25333, doi:10.1038/srep25333 (2016). Cappetta, D., Urbanek, K., Rossi, F. \& De Angelis, A. Anthracycline cardiotoxicity: new actors on the stage. Transl Cancer Res 7, S580-S583, doi:10.21037/tcr.2018.04.24 (2018). Chatterjee, K., Zhang, J. Q., Honbo, N. \& Karliner, J. S. Doxorubicin Cardiomyopathy. Cardiology 115, 155-162, doi:10.1159/000265166 (2010). FDA. <www.fda.gov/downloads/drugs/guidancecomplianceregulatoryinformation/guidances/ucm073395.pdf> (2012). Galvao, J. et al. Unexpected low-dose toxicity of the universal solvent DMSO. Faseb J 28, 1317-1330, doi:10.1096/fj.13-235440 (2014). Sumida, K. et al. Effects of DMSO on gene expression in human and rat hepatocytes. Hum Exp Toxicol 30, 1701-1709, doi:10.1177/0960327111399325 (2011). Yuan, Y. et al. Efficient long-term cryopreservation of pluripotent stem cells at-80 degrees C. Sci Rep-Uk 6, doi:ARTN 34476 10.1038/srep34476 (2016). perturb viability and fetal development. Reprod Fert Develop 17, 371-378, doi:10.1071/Rd04102 (2005). Benkhalifa, M. et al. Mitochondria: Participation to infertility as source of energy and cause of senescence. Int J Biochem Cell B 55, 60-64, doi:10.1016/j.biocel.2014.08.011 (2014).

Heijmans, B. T. et al. Persistent epigenetic differences associated with prenatal exposure to famine in humans. P Natl Acad Sci USA 105, 17046-17049, doi:10.1073/pnas.0806560105 (2008).

\section{Tobi, E. W. et al. DNA methylation differences after exposure to prenatal famine are common and} timing- and sex-specific. Hum Mol Genet 18, 4046-4053, doi:10.1093/hmg/ddp353 (2009). Veenendaal, M. V. E. et al. Transgenerational effects of prenatal exposure to the 1944-45 Dutch famine. Bjog-Int J Obstet Gy 120, 548-554, doi:10.1111/1471-0528.12136 (2013).

De Rycke, M., Liebaers, I. \& Van Steirteghem, A. Epigenetic risks related to assisted reproductive technologies - Risk analysis and epigenetic inheritance. Hum Reprod 17, 2487-2494, doi:DOI 10.1093/humrep/17.10.2487 (2002).

Hart, R. \& Norman, R. J. The longer-term health outcomes for children born as a result of IVF treatment: Part IGeneral health outcomes. Hum Reprod Update 19, 232-243, doi:10.1093/humupd/dms062 (2013). doxorubicin and mitoxantrone. Arch Toxicol 90, 2063-2076, doi:10.1007/s00204-016-1759-y (2016). Sorensen, J. C. et al. Mitochondria: Inadvertent targets in chemotherapy-induced skeletal muscle toxicity and wasting? Cancer Chemother Pharmacol 78, 673-683, doi:10.1007/s00280-016-3045-3 (2016). Burridge, P. W. et al. Human induced pluripotent stem cell-derived cardiomyocytes recapitulate the predilection of breast cancer patients to doxorubicin-induced cardiotoxicity. Nat Med 22, 547-556, doi:10.1038/nm.4087 (2016).

Yang, F., Teves, S. S., Kemp, C. J. \& Henikoff, S. Doxorubicin, DNA torsion, and chromatin dynamics. Biochim Biophys Acta 1845, 84-89, doi:10.1016/j.bbcan.2013.12.002 (2014). Edwardson, D. W. et al. Role of Drug Metabolism in the Cytotoxicity and Clinical Efficacy of Anthracyclines. Curr Drug Metab 16, 412-426 (2015).

Berthiaume, J. M. \& Wallace, K. B. Adriamycin-induced oxidative mitochondrial cardiotoxicity. Cell Biol Toxicol 23, 15-25, doi:10.1007/s10565-006-0140-y (2007).

Varga, Z. V., Ferdinandy, P., Liaudet, L. \& Pacher, P. Drug-induced mitochondrial dysfunction and cardiotoxicity. Am J Physiol Heart Circ Physiol 309, H1453-1467, doi:10.1152/ajpheart.00554.2015 (2015).

Tokarska-Schlattner, M., Zaugg, M., Zuppinger, C., Wallimann, T. \& Schlattner, U. New insights into doxorubicin-induced cardiotoxicity: The critical role of cellular energetics. J Mol Cell Cardiol 41, 389-405, doi:10.1016/j.yjmcc.2006.06.009 (2006). 
Wang, E. T. et al. Alternative isoform regulation in human tissue transcriptomes. Nature $456,470-476$, doi:10.1038/nature07509 (2008).

van den Hoogenhof, M. M. G., Pinto, Y. M. \& Creemers, E. E. RNA Splicing Regulation and Dysregulation in the Heart. Circ Res 118, 454-468, doi:10.1161/Circresaha.115.307872 (2016).

Richard, H. et al. Prediction of alternative isoforms from exon expression levels in RNA-Seq experiments. Nucleic Acids Res 38, doi:ARTN e112 10.1093/nar/gkq041 (2010).

de Souza, T. M. Bits and bytes in toxicogenomics: In silico approaches in liver-based mechanistic toxicology; Chapter 3. (2017).

Garfinkel, A. C., Seidman, J. G. \& Seidman, C. E. Genetic Pathogenesis of Hypertrophic and Dilated Cardiomyopathy. Heart Fail Clin 14, 139-146, doi:10.1016/j.hfc.2017.12.004 (2018).

Herman, D. S. et al. Truncations of Titin Causing Dilated Cardiomyopathy. New Engl J Med 366, 619-628, doi:DOI 10.1056/NEJMoa1110186 (2012).

Fatkin, D. \& Huttner, I. G. Titin-truncating mutations in dilated cardiomyopathy: the long and short of it. Curr Opin Cardiol 32, 232-238, doi:10.1097/Hco.0000000000000382 (2017).

Duchaine, T. F. \& Fabian, M. R. Mechanistic Insights into MicroRNA-Mediated Gene Silencing. Csh Perspect Biol, a032771 (2018).

Liu, J., Liu, T., Wang, X. \& He, A. Circles reshaping the RNA world: from waste to treasure. Mol Cancer 16, 58, doi:10.1186/s12943-017-0630-y (2017).

Qu, S. B. et al. Circular RNA: A new star of noncoding RNAs. Cancer Lett 365, 141-148, doi:10.1016/j.canlet.2015.06.003 (2015).

Memczak, S. et al. Circular RNAs are a large class of animal RNAs with regulatory potency. Nature 495, 333-338, doi:10.1038/nature11928 (2013).

Payne, S. H. The utility of protein and mRNA correlation. Trends Biochem Sci 40, 1-3, doi:10.1016/j.tibs.2014.10.010 (2015).

Chen, Z. J. et al. Fresh versus Frozen Embryos for Infertility in the Polycystic Ovary Syndrome. New Engl J Med 375, 523-533, doi:10.1056/NEJMoa1513873 (2016).

Maier, T., Guell, M. \& Serrano, L. Correlation of mRNA and protein in complex biological samples. Febs Lett 583, 3966-3973, doi:10.1016/j.febslet.2009.10.036 (2009).

Zhang, X. O. et al. Diverse alternative back-splicing and alternative splicing landscape of circular RNAs. Genome Res 26, 1277-1287, doi:10.1101/gr.202895.115 (2016).

Gao, Y., Zhang, J. \& Zhao, F. Circular RNA identification based on multiple seed matching. Briefings in bioinformatics, doi:10.1093/bib/bbx014 (2017).

Mohr, S. E. \& Perrimon, N. RNAi screening: new approaches, understandings, and organisms. Wires Rna 3, 145-158, doi:10.1002/wrna.110 (2012).

Fatehullah, A., Tan, S. H. \& Barker, N. Organoids as an in vitro model of human development and disease. Nat Cell Biol 18, 246-254, doi:DOI 10.1038/ncb3312 (2016). 

Valorization 
Valorization of scientific knowledge is "the process of creating value from knowledge, by making knowledge suitable and/or available for social and/or economic use and by making knowledge suitable for translation into competitive products, services, processes and new commercial activities" ${ }^{[1]}$. The opportunities for valorization of the current dissertation are described in this chapter.

\section{Relevance}

Drug development and safety assessment are long and costly processes, which are currently not able to eliminate all health risks related to drug use ${ }^{[2]}$. This thesis aimed to improve toxicological drug safety assessments by adopting an experimental in vitro design better reflecting the human in vivo conditions combined with advanced analysis of post-transcriptional mechanisms.

Because current in vitro toxicological drug safety assessment is unable to detect all drug-induced toxicities, many investigated candidate compounds fail during animal testing and never enter the market ${ }^{[3]}$. Animal experiments are time consuming, labor intensive and very expensive ${ }^{[4]}$. Therefore, failure of a candidate compound due to toxicities is a waste of money and resources which can be prevented through improved in vitro assays. But even when a candidate compound passes all stages of the safety assessment, side effects or adverse drug reactions can occur due to undetected toxicities. Animal experiments are usually performed using mice or rats and toxicity results are extrapolated to predict human toxicities ${ }^{[5]}$. Overall, the accuracy of human risk prediction using rodent experiments is only $50-70 \%$, which is barely better then flipping a coin ${ }^{[6]}$. The adverse drug reactions are a major cause for regulatory actions like restrictions on drug use in the form of black box warnings or even drug withdrawal from the market ${ }^{[7-9]}$. This again results in an economical burden in the form of money and resources squandered, but also increase ethical objections to animal testing. More important is the societal impact of incorrect human risk assessments because drug induced toxicities rank high as cause of disease and death ${ }^{[10]}$. This clearly indicates that there is need for improvement of drug safety testing, to which the results in this thesis contribute. 


\section{Target Groups}

While the outcome of this thesis may be relevant for a diverse range of scientific fields, we focus on the field of toxicology. Target groups for whom the outcome of this thesis may be of interest include regulatory agencies, industry and ultimately the general population.

Specialized regulatory organizations (i.e. U.S Food and Drug Administration; FDA) are responsible for the safety of pharmaceuticals, food-additives and chemicals. For monitoring purposes, strict guidelines are developed which contain regulations and recommendations regarding the safety testing procedure ${ }^{[11,12]}$. Before new in vitro test methods can be implemented in toxicological safety assessments, they need to be approved by these regulatory agencies. In this context, the models and methods described in this thesis may be of interest to these regulatory organizations.

All branches of industry dealing with toxicological testing of compounds may benefit from our results. While this also includes manufactures of food products and cosmetics, the highest relevance will be for the chemical and pharmaceutical companies. The ability to reliably predict human risk in vitro will have tremendous benefits for these industries. Because the toxicity of a candidate compound can be assessed during early phases of the drug development process, money and resources (including animals) can be saved for compounds with actual therapeutic potential. Furthermore, this would also lead to fewer animal experiments. Therefore, the results in this thesis also contribute towards the replacement, reduction and refinement of animal testing ${ }^{[13]}$, which eases the societal resistance against animal testing. Furthermore, reliable risk assessment would prevent incorrect conclusions. This not only prevents costly drug withdrawal from the market, but also prevents compounds from being wrongfully classified as toxic. These additional non-toxic compounds with therapeutic potential increases the chance of finding new treatments. Overall, better in vitro testing methods for toxicological assessment, would make the drug development and safety 
assessment processes more efficient and cheaper. Subsequently, prices could be decreased for medication and health care in general, which could the general population.

\section{Activities and Innovation}

In the first part of this thesis, we described the use of 3D cell cultures exposed to PBPK-based dosing profiles to reflect more accurately the in vivo human responses to a toxicant. Current toxicological exposures are usually performed using a stable toxicant dose, often at higher concentrations than found in vivo to strengthen the toxic effects for investigation. In Chapter 3 we showed the difference in cellular responses between the therapeutic and the toxic dosing profile of doxorubicin, thereby highlighting the importance of investigating relevant in vivo therapeutic doses during toxicological assesments. Additionally, our innovative experimental design incorporates influences of toxicant absorption, distribution, metabolism and excretion through changing of toxicant doses (three times daily) based on PBPK-models. While this procedure increases the labor intensity of in vitro exposures, it highly contributes to creating resemblance of in vitro models to in vivo situations. Therefore, the accuracy of in vitro human toxicological risk assessment could be increased by adopting such in vitro models. Furthermore, in Chapter 2 we evaluated the effect of $0.1 \%$ DMSO exposure and showed for the first time that DMSO does not only alter gene expression, but also more strongly influences DNA methylation patterns of human cells in vitro. While a concentration of $0.1 \%$ DMSO is generally viewed as a low concentration, it is actually extremely high compared to physiologically relevant in vitro toxicant doses. For example, the maximum toxicant concentration used in the therapeutic DOX-dosing profile applied in this thesis was $0.22 \mu \mathrm{M}$, while $0.1 \%$ DMSO equals a concentration of 14.1 $\mathrm{mM}$ ! This implies that the generally used concentration of $0.1 \%$ DMSO which may pose a threat in generating erroneous conclusions gained from in vitro toxicity assessments (i.e. false negative drug toxicity conclusions). Therefore, use of DMSO during in vitro assays should be avoided or reduced where possible. 
The second part of this thesis focused on using high throughput sequencing and advanced data-analysis to obtain a deeper understanding of drug-induced toxicity. While the use of sequencing technology to analyze changes in gene expression already enables the complete monitoring of toxicant-induced effects in a cell, there is more detailed information to be gained from RNA sequencing data through incorporating advanced data-analysis of post-transcription mechanisms.

In Chapter 4 we showed the added value of analyzing toxicant-induced changes due to alternative splicing through which one gene can encode a great variety of transcripts which differ in biological functions as well as their ability to be translated into a protein. During the analysis of anthracycline- exposed samples, we observed changes in ratios of expressed transcripts (defined as differential transcript usage; DTU) which entailed a switch from a protein-coding transcript to a non-protein coding transcript. While gene expression was not significantly changed, the observed decrease in protein coding transcripts implied a decrease of protein content which may contribute to the cardiotoxic effects of anthracyclines. Therefore, analysis of DTU provides an extra layer of detail able to identify toxicant-induced changes, which would have been overlooked when solely analyzing gene expression changes. Furthermore, in Chapter 5 we designed a scoring system to identify changes in the post-transcriptional microRNA-induced inhibition of translation. The scoring system was designed based on mRNA, microRNA and circular RNA expression values and predicted interactions.

Compared to unadjusted RNA counts, estimated translatable mRNA read counts gained using the scoring system correlate better with the obtained proteome data, which are the functional enteties of the cell. Although the results of this analysis are still preliminary, this suggests the potential of applying the scoring system to the whole transcriptome in order to estimate the protein abundance of proteins which could not be measuered using proteomics techniques due to its detection limit. This would result in a complete dataset which is more relevant for evaluating functions and the investigated phenotype. 
These new approaches incorporating assessment of post-transcriptional mechanisms should service the further enrichment of analytical tools that will become necessary in future research to make optimal use of sequencing data to allow a better understanding of which compounds cause toxicity and what mechanisms are affected.

\section{Implementation}

In this thesis we described an experimental design better reflecting the human in vivo conditions combined with advanced analysis of post-transcriptional mechanisms. Using these models and methods, an increased understanding of cellular processes affected by a toxicant can be obtained. This information may be applied for the identification of novel biomarkers for early detection of toxicity or even to identify new treatment targets to prevent toxic phenotypes. However, it would be more beneficial to incorporate this more accurate in vitro test directly into safety testing procedures to more reliably predict the in vivo human responses to a toxicant. Though the research community has embraced the power of high throughput omics analysis, regulatory agencies have not yet acknowledged its value for toxicological assessments. Regulators still remain skeptical about the reliability and robustness of data gained from omics analysis, mainly due to inter-platform variations and bioinformatics pipelinedependent differences. In order to address these issues, a new project has been created: "Towards the Development of an Omics Data Analysis Framework (ODAF) for Regulatory Application" (CEFIC-LRI-C4) ${ }^{[14]}$. In this project, we will develop a regulatory ODAF (R-ODAF) to enable the regulatory bodies to consider omics data as a relevant data type for the purpose of supporting toxicological drug safety assessments. 


\section{References}

$1 \quad$ Maastricht promotie regelement. (2018).

2 Verheijen, M., Krauskopf, J., Kleinjans, J. C., de Kok, T. M. \& Caiment, F. Development and regulatory application of microRNA biomarkers. Biomark Med 9, 1137-1151, doi:10.2217/bmm.15.50 (2015).

Roberts, R. A. et al. Reducing attrition in drug development: smart loading preclinical safety assessment. Drug discovery today 19, 341-347, doi:10.1016/j.drudis.2013.11.014 (2014). Levy, N. The use of animal as models: ethical considerations. Int J Stroke 7, 440-442, doi:10.1111/j.17474949.2012.00772.x (2012).

5 Walmsley, R. M. \& Billinton, N. How accurate is in vitro prediction of carcinogenicity? British Journal of Pharmacology 162, 1250-1258, doi:10.1111/j.1476-5381.2010.01131.x (2011).

Olson, H. et al. Concordance of the toxicity of pharmaceuticals in humans and in animals. Regul Toxicol Pharm 32, 56-67, doi:10.1006/rtph.2000.1399 (2000).

$7 \quad$ Chen, M. et al. Toward predictive models for drug-induced liver injury in humans: are we there yet? Biomark Med 8, 201-213, doi:10.2217/bmm.13.146 (2014). Osaki, M., Kosaka, N., Okada, F. \& Ochiya, T. Circulating microRNAs in drug safety assessment for hepatic and cardiovascular toxicity: the latest biomarker frontier? Mol Diagn Ther 18, 121-126, doi:10.1007/s40291-013-0065-0 (2014). Hornberg, J. J. et al. Exploratory toxicology as an integrated part of drug discovery. Part I: Why and how. Drug discovery today 19, 1131-1136 (2014). Hornberg, J. J. et al. Exploratory toxicology as an integrated part of drug discovery. Part II: Screening strategies. Drug discovery today 19, 1137-1144, doi:10.1016/j.drudis.2013.12.009 (2014). Guidance on REACH (Registration Evaluation Authorization and restriction of Chemicals), $<$ https://echa.europa.eu/guidance-documents/guidance-on-reach> (accessed on Oct 2018). (Organisation for Economic Co-operation and Development) OECD Test Guidelines for the Chemicals, <http://www.oecd.org/chemicalsafety/testing/oecdguidelinesforthetestingofchemicals.htm> (accessed on Oct 2018). Baumans, V. Use of animals in experimental research: an ethical dilemma? Gene therapy 11, S64 (2004). Caiment, F. LRI C4 - Towards the Development of an Omics Data Analysis Framework (ODAF) for Regulatory Application, <http://cefic-Iri.org/request-for-proposals/Iri-c4-towards-the-development-ofan-omics-data-analysis-framework-odaf-for-regulatory-application/> (accessed on Oct 2018). 


\section{ADDENDUM II}

Ahout the author 
I, Marcha Verheijen, was born on March 25th 1987 in Amersfoort, the Netherlands. In high school, at the age of 14 , my fascination with genetics started. After high school, from 2003 till 2007, I continued my studies at Leeuwenborgh Sittard. Because chemistry is at the basis of all biological reactions and most laboratory techniques, I choose to study 'Chemical Laboratory Techniques'. Thereafter, I returned to biology during my bachelor of applied sciences in 'Biology and Medical Laboratory

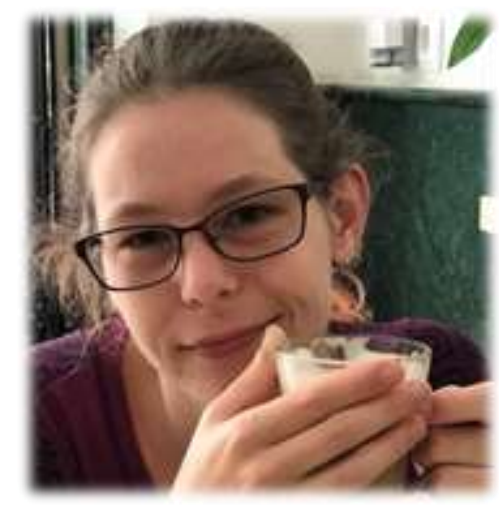
Research' at Hogeschool Zuyd Heerlen. By putting in some extra effort, I shortened my education to just over 3 years. I received my diploma in October 2010. At this moment in time, I had to decide whether it was my ambition to be a laboratory technician or if I preferred to continue my studies by obtaining a master's degree. Since classes at the university had already started, I was not in a hurry to decide. In the meantime, PathoFinder BV, where I did one of my internships, offered me a temporary job to help out during their busy season, which I gladly accepted. During the 3 months working in this company, I realized that I would rather be actively involved in research projects, instead of only executing laboratory tests. Therefore, I signed up for a master's program starting in September 2011. To pass the time, I got another temporary job (replacement for maternity leave) at the department of hereditary metabolic diseases at the academic hospital in Maastricht (AZM). Finally, I could start my masters study 'Biomedical Sciences' at Maastricht University with the specialization 'Clinical Molecular Sciences'. I did my first internship at the department toxicology focusing on 'transgenerational effects of paternal benzo[a]pyrene exposure and activation of retrotransposable elements' and my second at the department clinical genomics researching 'Mitochondrial dysfunction and genetics in Renal Cell Carcinoma'. After obtaining my master's degree in 2013, I set out to find the perfect PhD project. During my search, I occupied myself with online courses, studying genetics \& evolution, bioinformatics and python programming. On the 14th of April 2014, I started working as a PhD-student in the area toxicogenomics on the project entitled: HeCaToS, which resulted in the current thesis entitled "Transcriptomics close to my heart: Advanced models \& methods for toxicogenomics research illustrated by anthracycline-induced cardiotoxicity". Directly after finishing my thesis, on $14^{\text {th }}$ of October 2018 , I continued my scientific carrier as a postdoc working on the CEFIC project "Towards the Development of an Omics Data Analysis Framework (ODAF) for Regulatory Application". 
ADDENDUM III

List of publications 


\section{Full papers}

- DMSO induces drastic changes in human cellular processes and epigenetic landscape in vitro.

M. Verheijen, M. Lienhard, Y. Schrooders, O. Clayton, R. Nudischer, S. Boerno, B.

Timmermann, N. Selevsek, R. Schlapbach, H. Gmuender, S. Gotta, J. Geraedts,

R. Herwig, J. Kleinjans, and F. Caiment.

Scientific Reports (2019). IF: 4.12

- Bringing in vitro analysis closer to in vivo: Studying doxorubicin toxicity and associated mechanisms in 3D human microtissues with PBPK-based dose modelling. M. Verheijen, Y. Schrooders, H. Gmuender, R. Nudischer, O. Clayton, J. Hynes, S. Niederer, H. Cordes, L. Kuepfer, J. Kleinjans, F. Caiment. Toxicology letters (2018). IF: 3.86

- Development and regulatory application of microRNA biomarkers. M. Verheijen, J. Krauskopf, J. Kleinjans J, T. de Kok T, F. Caiment. Biomarkers in Medicine (2015). IF: 2.02

- Effects of benzo[a]pyrene on mouse germ cells: heritable DNA mutation, testicular cell hypomethylation and their interaction with nucleotide excision repair.

R. Godschalk, N. Verhofstad, M. Verheijen, C. Yauk, J. Linschooten, H. van Steeg, C. van Oostrom, J. van Benthemc, F. van Schootena.

Toxicology Research (2015). IF: 1.97

\section{Abstracts}

- Revealing DMSO-induced Bias In Omics Analysis In Vitro M. Verheijen, M. Lienhard, Y. Schrooders, O. Clayton, R. Nudischer, S. Boerno, N. Selevsek, H. Gmuender, R. Herwig, J. Kleinjans, F. Caiment. HeCaToS meeting 2017, Valencia (SP); GROW science day 2017, Maastricht (NL); SOT Society of Toxicology (SOT) 2018, San Antonio, Texas (USA)

- Doxorubicin-induced cardiotoxicity in 3D human microtissues using PBPK-based dose modelling

M. Verheijen, Y. Schrooders, H. Gmuender, R. Nudischer, O. Clayton, J. Hynes, S. Niederer, H. Cordes, L. Kuepfer, J. Kleinjans, F. Caiment.

HeCaToS meeting 2017, Valencia (SP); GROW science day 2017, Maastricht (NL)

- Time-series analysis - Doxorubicin Mitochondrial Model

M. Verheijen, Y. Schrooders, J. Kleinjans, F. Caiment

HeCaToS meeting 2016, Valencia (SP); GROW science day 2016, Maastricht (NL); The Netherlands Society of Toxicology (NVT), June 2016, Soesterberg (NL)

- Transcriptomics in the HeCaToS project

M. Verheijen, F. Caiment, Y. Schrooders, T. vd Beucken, J. Briede, J. Kleinjans HeCaToS meeting 2015, Valencia (SP); GROW science day 2015, Maastricht (NL) 


\section{ADDENDUM IV}

Acknowledgements 
This is the last chapter of my thesis, which marks the end of an era. These last 4.5 years were filled with fun and frustration. The frustrations made me stronger and hopefully a better scientist. The fun I will remember for the rest of my life. I was very fortunate to have people in my life to share these moments with. I hope that these people already know that I appreciated all they did for me. Just in case, I would like to express my gratitude with a few simple words to all that have aided me during my PhD.

\section{Thanks to all of you!}

My time as a DhD-student would not have been the same without you! 
I would like to use these last pages of my thesis to specially thank the people on which I relied most during my PhD.

First, I would like to express my gratitude to my promotion team.

Prof. dr. Jos Kleinjans: Thank you for hiring me as a PhD-student and giving me the opportunity to be part of the HeCaToS project. You not only educated me in the field of science, but you also made me grow as a person. You made me find my confidence and helped to overcome my fears (in the beginning, you terrified me every time you summoned me to your office).

Dr. Florian Caiment: You were the best supervisor I could hope for! You were always polite and friendly to me, even when I made very stupid mistakes. You were always willing to share you expert knowledge (and very driven and detailed when doing so). I think we make an effective team, even though we developed a tendency to digress to personal conversations during our meetings. I am grateful that you were willing to guide me, and overjoyed that you hired me as your postdoc.

Second, I would like to thank all the members of the HeCaToS-team for their contributions and the fun times in Valentia. Special thanks go out to Olivia, for

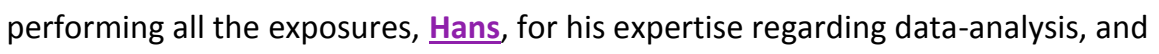
Matthias, for his epigenomics contribution to chapter 2.

Yannick, my young padawan, you have become the master of library preparations. Without you, I would not have the data used for this thesis. Like your Pokémon's, I hope you will be able to catch all of your dreams.

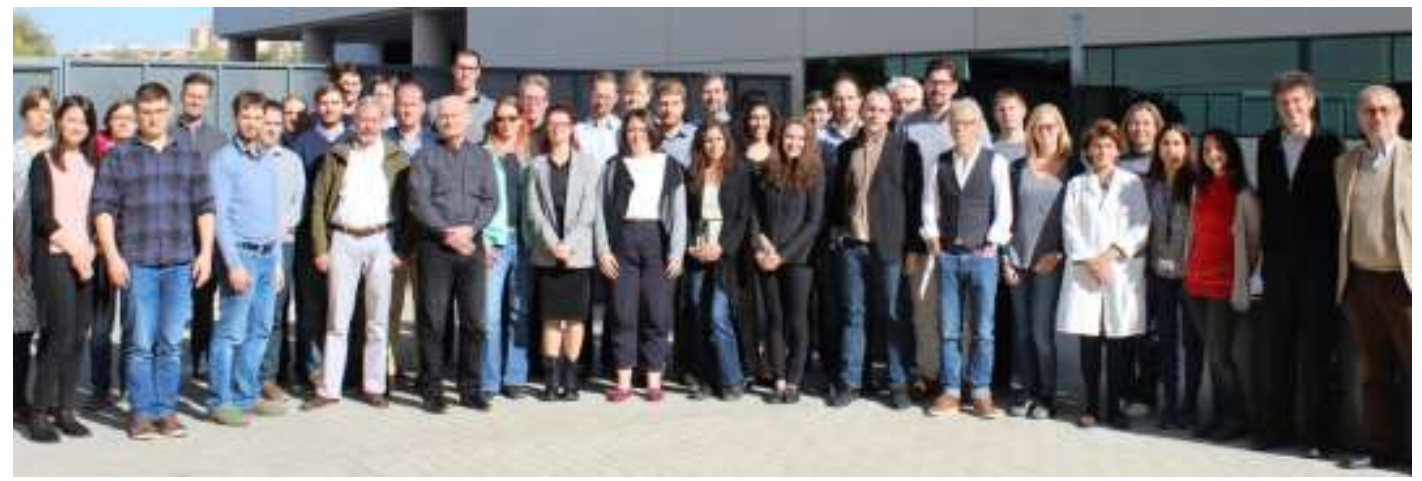

Group picture of the HeCaToS team members present at the Valencia meeting 2017 
Third, I turn the focus to my roommates. First: $\underline{\text { Héloïse, Julian and Almudena, }}$ thereafter: Daniela and Nhan. And maybe I should also include Marlon (you visited so often that you felt like a roommate). We tossed around ideas just (like we did balls), prayed that we would survive our PhDs (unlike our water dragons) and that our thesis would grow better than our in office greenhouse experiment. Because of each and every one of you, our office was a safe haven. We laughed and we cried and we got each other's backs. I am forever grateful for the support you gave me.

Of course, my room mates were not the only ones I had fun with. Therefore, I would also like to thank the other PhD students (present and graduated) and postdocs for the useful discussions and very nice times (such as bowling, game nights and ladies nights).

And finally, I saved the best for last, I would like to thank my family and paranymphs.

Mom \& dad: Even though I had to do this adventure on my own, you were the ones who made this possible. You always supported me and provided guidance when I needed it most. I am forever grateful and I love you very much!

Ruud: Too bad that you had to miss the beginning of this adventure, but your support helped me reach the finish line. I am sorry for bothering you with all my whining. You were my shoulder to cry on, my helping hand and the kick underneath my ass when I needed it. With you, I stand strong. I can conquer the world with one hand, as long as you are holding the other. Now and forever!
Pap \& mam: Hoewel dit mijn eigen avontuur was, had ik het niet zonder jullie kunnen doen. Jullie hebben me altijd gesteund en gaven me advies wanneer ik dit het hardste nodig had. Ik ben jullie voor eeuwig dankbaar en ik hou heel veel van jullie!

Ruud: Het begin van dit avontuur heb je jammer genoeg gemist, maar je steun heeft me wel naar de eindstreep geholpen. Sorry dat je al mijn gezanik en gezeur hebt moeten aanhoren. Jij was mijn schouder om op te huilen, mijn steuntje in de rug en de schop onder mijn kont wanneer ik die nodig had. Met jou, sta ik sterk. Met jou kan ik de hele wereld aan. Nu en voor altijd! 
Leny: In the final stage, you relieved the burden by cooking for me. Your delicious dishes gave me the energy I needed to finish my thesis.

Rianne: My big sister, you have been by my side my whole life. We protect each other like an impenetrable wall. Though we chose completely different careers (you becoming an IT teacher and me becoming a biological researcher), we are more alike than we sometimes like to admit. As we found out these last years, when I helped you with research during your master and you helping me with programming during my PhD. And you are not just my sister, you are also my best and closest friend. Therefore, I also want you by my side at the defense as my paranymph.
Leny: Tijdens de laatste fase verlichtte jij de last door voor mij te koken. Jou heerlijke gerechten gaven mij de energie die ik nodig had om mijn proefschrift af te ronden.

Rianne: Mijn grote zus, al mijn hele leven sta je aan mijn zijde. We beschermen elkaar als een ondoordringbare muur. Hoewel we zeer verschillende carrières hebben gekozen (jij ICT docent en ik biologisch onderzoeker), lijken we meer op elkaar als dat we soms willen toegeven. Dit hebben we de afgelopen jaren wel ondervonden, toen ik jou hielp met onderzoek tijdens je master en jij mij hebt geholpen met programmeren tijdens mijn PhD. En je bent niet alleen mijn zus, je bent ook mijn beste en dierbaarste vriendin. Daarom heb ik je ook graag aan mijn zijde als paranimf tijdens mijn verdediging.

Héloïse: We rode this rollercoaster together. You saw it all, the good and the bad. Through it all, we stuck together. That is why I want you by my side at my defense, as my paranymph and my friend. I found this poem that describes my gratitude better than I can. In hard times that seem to have no end

In hard times that seem to have no end
Nothing beats the thoughtfulness of an extraordinary friend
Your lind heart and helping hand
Along with patient listening and ability to understand
Xade a burden I thought was impossible to bare
Fade into something conquerable
Because you were there!

\section{Thanks to all who stood by me!}

